\title{
RAINFALL IN VIETNAM
}

by

Nguyen Dang Quang

\author{
A thesis \\ submitted to Victoria University of Wellington in \\ fulfillment of the requirements for the degree of \\ Doctor of Philosophy \\ in Geophysics
}

Victoria University of Wellington

2015 


\section{ACKNOWLEDGEMENTS}

To my advisors, thank you. James McGregor and James Renwick have fully supported me throughout this journey. From their deep intellectual insight and experience they have given fair comments and encouraged healthy discussion. Time and again I learnt lessons from these two men. For James McGregor I will never forget the way he looks at a problem, trying to reveal some hidden angles and perspectives. He reminded me to broaden things from preliminary results. For James Renwick, the man I met a while before I came to New Zealand to work under his supervision, my first feelings about him was that he was a great supporter in my field of study. "You can do it, so do it", are some words that stimulated me, giving me more confidence to overcome difficult times during my Ph.D work.

It is my pleasure to thank the New Zealand Development Scholarships (NZDS) programme for their full financial support, the Victoria International team, and the School of Geography, Environment, and Earth Sciences for their great management. They could not do a better job.

Thanks go to my colleagues in the Vietnam National Centre for Hydrometeorological Forecastings. They encouraged me from the beginning when I submitted the NZDS application through to the very end of the journey.

Lastly, special recognition goes out to my beloved wife Trang, and our two wonderful children Linh and Khoi for being with me during the entire process. They balance my life between books, programming codes, sleepless nights, and everyday life. Born in New Zealand, Khoi has to be one of my sweetest New Zealand souvenirs ever. 


\section{ABSTRACT}

The thesis is a comprehensive analysis of the climate of Vietnam, concentrating on rainfall. Vietnam lies in the tropical northern hemisphere in a region that is influenced by the South Asian, East Asian and Australian monsoons. Rainfall here is associated with several different mechanisms, such as the monsoon, tropical cyclones, topography, and so on. From the initial climatological analysis, monsoon and non-monsoon rainfall is investigated. Non-monsoon rainfall is found to be related to a vortex often found off the coast of Vietnam, a newly-understood extension of the "Borneo vortex".

The thesis begins with a study of the surface climate and the general atmospheric circulation over the Southeast Asian region, which dominates Vietnam's climate. Trends of surface temperature and rainfall were investigated for a 40 year period (1971-2010), using a newly-extended dataset of 60 stations. Vietnam's average temperature has increased at a rate of $0.26 \pm 0.10^{\circ} \mathrm{C}$ per decade since the $1970 \mathrm{~s}$, approximately twice the rate of global warming over the same period. The increase in temperature is statistically significant in most sub-regions. Trends in rainfall are however mostly insignificant in that period. Temperature and rainfall variability are shown to be linked to ENSO on both a national and sub-regional scale. The rainfall climate of Vietnam has been studied in depth, particularly the characteristics of monsoon rainfall and the variability of the length of wet and dry seasons. That investigation has led to the development of an objective monsoon index, based on regional mean sea level pressure and low level zonal wind. While originally developed to define onset and withdrawal dates for the monsoon in Vietnam and over Southeast Asia, the index is shown to be applicable in all monsoon regions of the globe, the first objective index to have such global utility. The final part of the thesis deals with tropical vortex activity and its associated rainfall. Vortices exist almost year-round, migrating from the coast of Vietnam - Southeast Asian Sea - Philippines in summer to the Borneo Island region in winter. Clear evidence is found for the presence of a semipermanent vortex near the south coast of Vietnam or the north of the Southeast Asian Sea - Maritime Continent (SEASMC) region. It is this vortex and the tropical cyclones over 
the SEASMC region that significantly contribute to the Vietnam rainfall, particularly the post-monsoon rainfall in Central Vietnam. 


\section{CONTENTS}

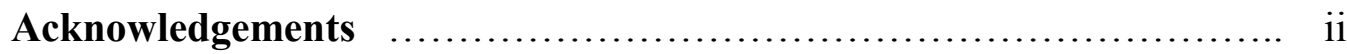

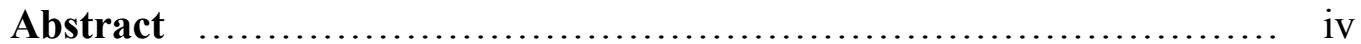

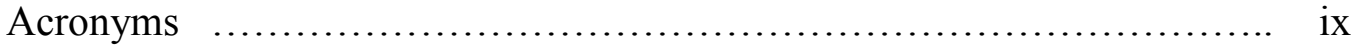

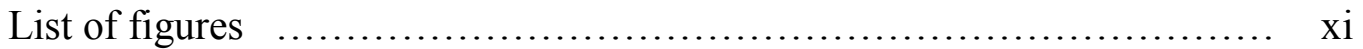

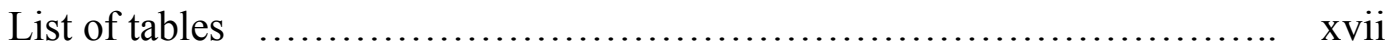

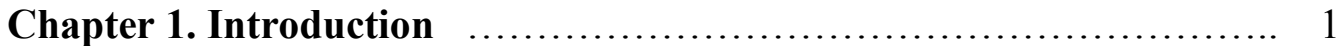

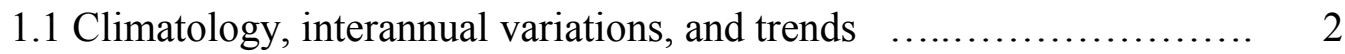

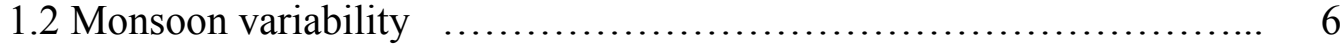

1.3 Tropical cyclones activity and their associated rainfall $\ldots \ldots \ldots \ldots \ldots . . . . . . \quad 7$

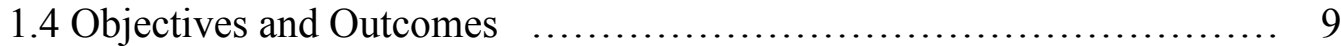

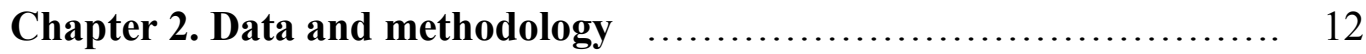

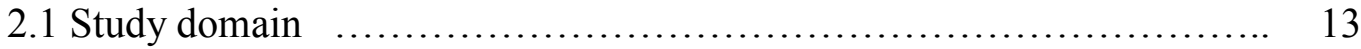

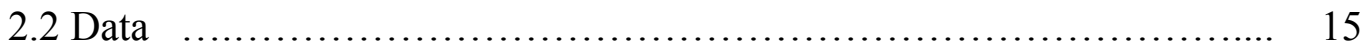

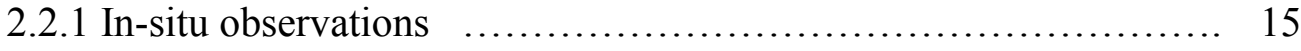

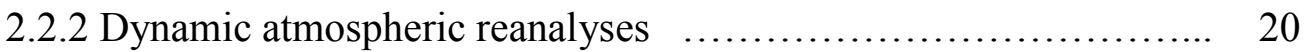

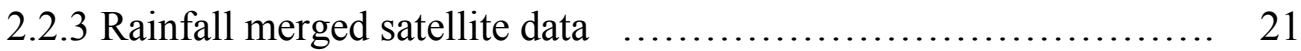

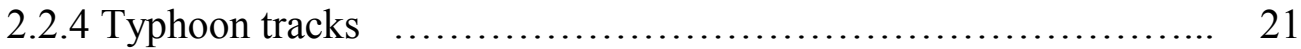

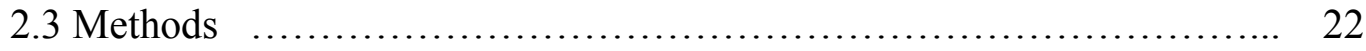

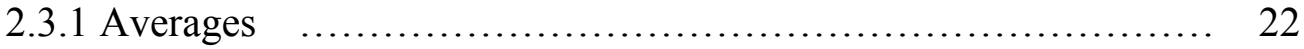

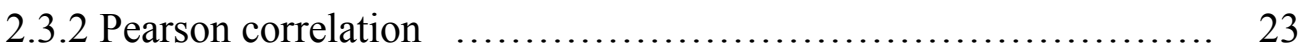

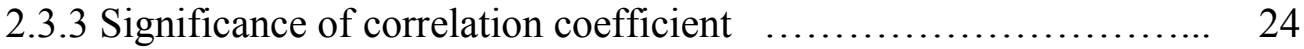

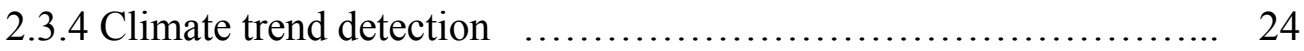

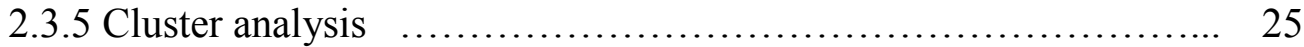

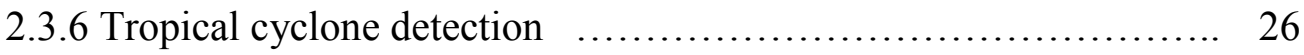

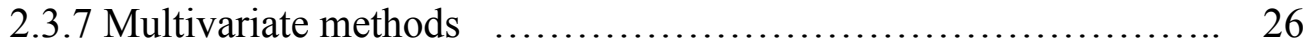

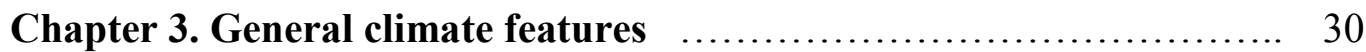

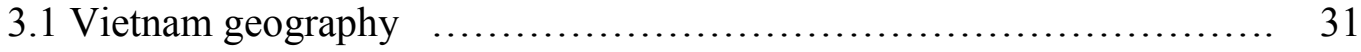

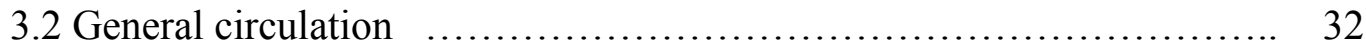




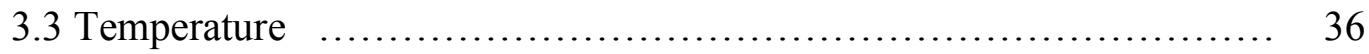

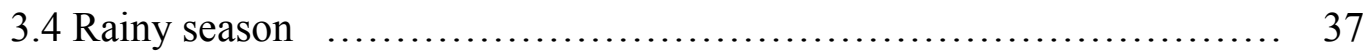

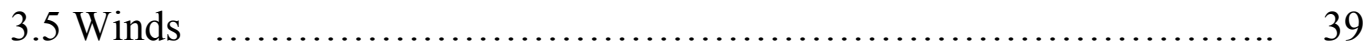

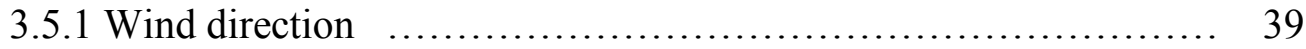

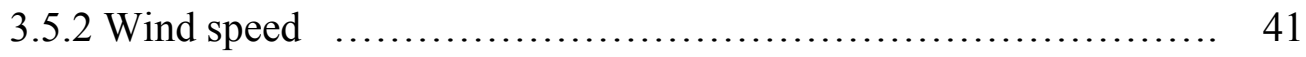

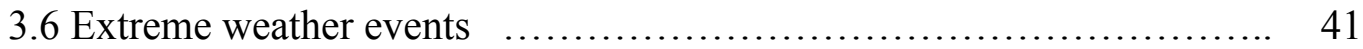

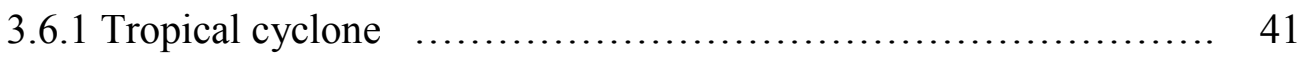

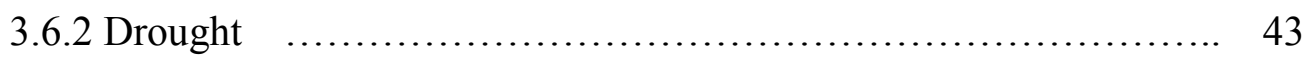

3.6.3 Flood .................................................... 44

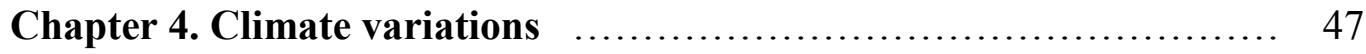

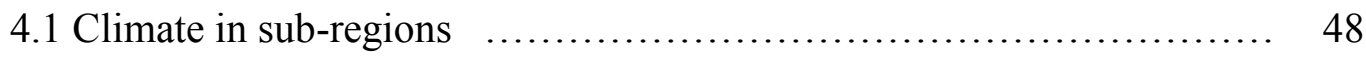

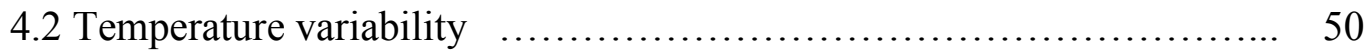

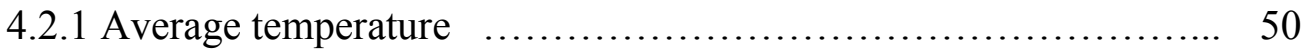

4.2.2 Maximum and minimum temperatures $\ldots \ldots \ldots \ldots \ldots \ldots \ldots \ldots \ldots . . \ldots \ldots$

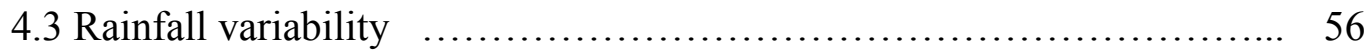

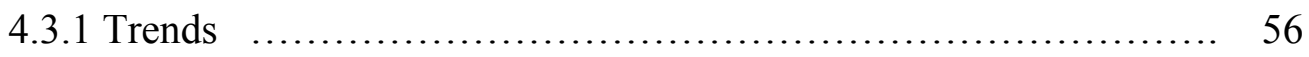

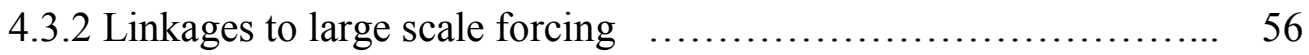

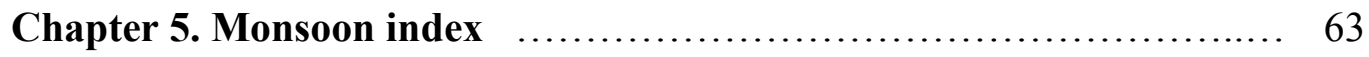

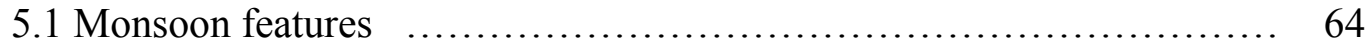

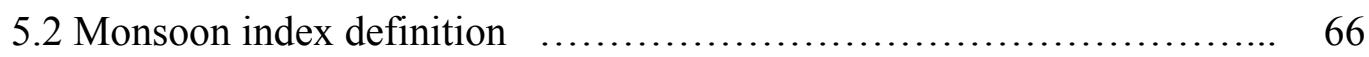

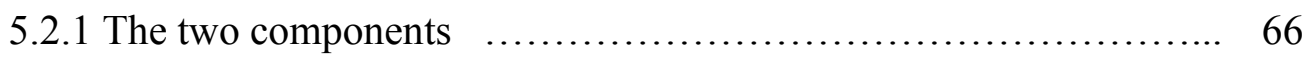

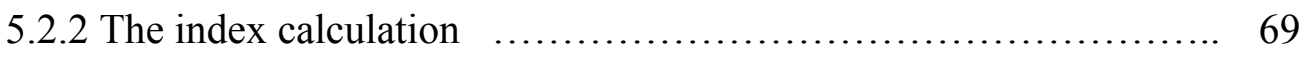

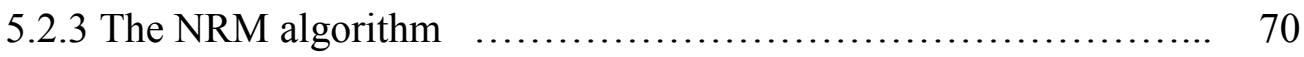

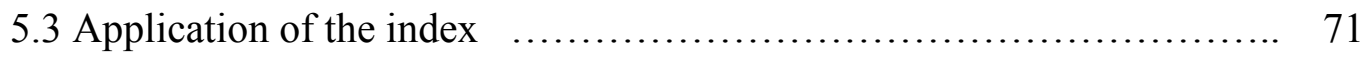

5.3.1 OD-WD in Vietnam and the Southeast Asia Sea region $\quad . . . \ldots \ldots \ldots . \quad 72$

5.3.2 Trends of changes in daily rainfall in Vietnam ................... 74

5.3.3 OD-WD in other major monsoon regions $\quad \ldots \ldots \ldots \ldots \ldots \ldots \ldots \ldots . . \ldots \ldots$

Chapter 6. Tropical vortex and its associated rainfall $\ldots \ldots \ldots \ldots \ldots \ldots \ldots . \ldots$ 


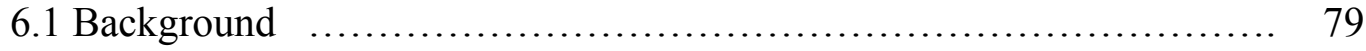

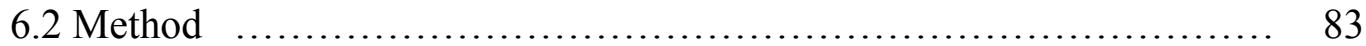

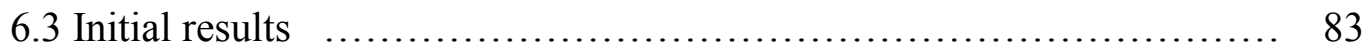

6.4 Southeast Asian Sea - Maritime Continent vortex $\quad \ldots \ldots \ldots \ldots \ldots \ldots \ldots . . \ldots 1$

6.5 Vortex activity and post-monsoon rainfall $\ldots \ldots \ldots \ldots \ldots \ldots \ldots \ldots . \ldots . \ldots . \ldots$

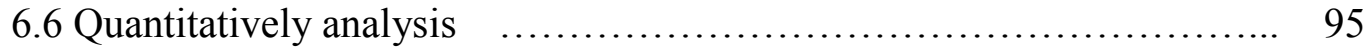

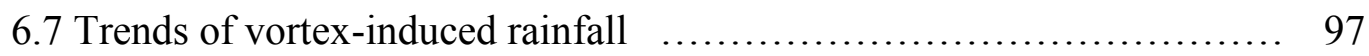

Chapter 7. Discussions and conclusions $\ldots \ldots \ldots \ldots \ldots \ldots \ldots \ldots \ldots \ldots \ldots, 100$

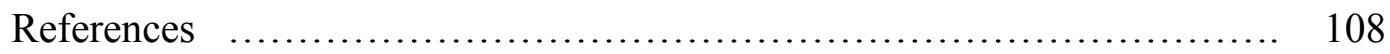

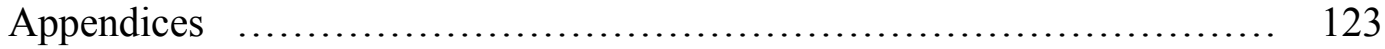

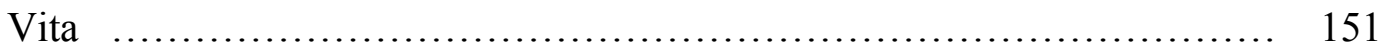




\section{ACRONYMS}

4D-VAR: Four Dimensional Variational Assimilation

APHRODITE: Asian Precipitation-Highly Resolved Observational Data Integration Towards Evaluation of Water Resources

CMAP: Climate Prediction Center Merged Analysis of Precipitation

DJF: December-January-February

ECMWF: European Centre for Medium Range Weather Forecasts

ENSO: El-Niño Southern Oscillation

EOF: Empirical Orthogonal Function

ERA - Interim: The latest ECMWF global atmospheric reanalysis of the period from 1979

ITCZ: Inter-tropical Convergence Zone

JCDAS: JMA Climate Data Assimilation System

JJA: June-July-August

JRA-25: Japanese 25-year Reanalysis (1979-2010)

JRA-55: Japanese 55-year Reanalysis (1979-2010)

IBTrACS-WMO: the International Best Tracks Archive for Climate Stewardship - WMO

MAM: March-April-May

MC: Maritime Continent.

MSLP: Mean Sea Level Pressure

NCEP: National Centers for Environment Prediction, United States 
NCEP1: The first NCEP Reanalysis Generation

NCEP-DOE: NCEP- Department of Energy, United States

NCHMF: Vietnam National Centre for Hydrometeorological Forecastings

NetCDF: Network Common Data Format

Niño 3.4 Index: SSTA over the region $\left(5^{\circ} \mathrm{N}-5^{\circ} \mathrm{S}, 170^{\circ} \mathrm{W}-120^{\circ} \mathrm{W}\right)$

NOAA: National Oceanic and Atmospheric Administration, United States.

NRM: Nguyen-Renwick-McGregor Monsoon Index

OLR: Outgoing Longwave Radiation

SEAMC: Southeast Asian Sea - Maritime Continent

SEAMCV: Southeast Asian Sea - Maritime Continent Vortex

SEAS: Southeast Asian Sea

SON: September-October-November

SSTA: Sea Surface Temperature Anomaly

SVD: Singular Value Decomposition Analysis

TC: Tropical Cyclone

TMD: Thai Meteorological Department

U850(U200): Zonal wind at 850(200)hPa

VR: Vortex-induced rainy days

WMO: World Meteorolgical Organisation

Z500: Geopotential Height Anomaly at 500hPa 


\section{List of Figures}

Figure

Page

1.1 Natural geographical map of Vietnam. Rivers (mountains) are represented by light green (dark yellow). The darker the blue, the deeper the sea floors over the Southeast Asian Sea

1.2 a) Lead surface synoptic-scale weather systems in summer in the Southeast Asian - Maritime Continent (SEAMC) region. Monsoon trough/ITCZ is displayed by the bold-dash line (Ding and Sikka, 2006). b) Same as a), except for winter (Johnson and Houze, 1987)

1.3 Monthly mean climatology of Vietnam rainfall (averaged 1979-2007). Contour interval: $3 \mathrm{~mm} \cdot$ day $^{-1}$ (Yen et al., 2011)

1.4 Track and intensity of all tropical storms, 1851-2006. The diagram uses the Saffir Simpson hurricane intensity scale. Vietnam is highlighted by the yellow box

2.1 Domains used in the study. REG1 $\left(50^{\circ} \mathrm{S}-70^{\circ} \mathrm{N} ; 40^{\circ} \mathrm{E}-120^{\circ} \mathrm{W}\right)$, REG2 $\left(7.5-25^{\circ} \mathrm{N} ; 100-120^{\circ} \mathrm{E}\right)$, REG3 $\left(10^{\circ} \mathrm{S}-25^{\circ} \mathrm{N} ; 100-120^{\circ} \mathrm{E}\right)$, and Global Tropical Monsoon $\left(40^{\circ} \mathrm{S}-40^{\circ} \mathrm{N} ; 0-360^{\circ}\right)$

2.2 Sixty Vietnamese observation stations used in the study. N1, N2, N3, N4 (S1, S2, S3) stands for Northern (Southern) climate sub-regions. Central region is considered to be the combination of N4 and S1 subregions. Truong-Sa $\left(8.7^{\circ} \mathrm{N}, 111.9^{\circ} \mathrm{E}\right)$ is the farthest offshore station

2.3 Anscombe's quartet. Sample diagrams in Pearson correlation analysis ... 23

2.4 Basic K-means algorithm ............................................................ 25

2.5 A demonstration of EOF analysis $\quad$..................................................... 28

3.1 Monthly long-term average temperature (dashed black line) and rainfall (solid red line) in Vietnam

3.2 Velocity potential ( $1 \mathrm{e} 6 \mathrm{~m}^{2} / \mathrm{s}$, contours) and divergent component of vector winds at $850 \mathrm{hPa}$ (bottom) and $200 \mathrm{hPa}$ (top). JCDAS 1979-2010 average for JJA (left) and DJF (right). Gray contours: Streamlines .....

3.3 Surface analysis map of a typical cold surge hitting Vietnam and the 
SEAS region on 00UTC 16 December 2009. Blue contours: surface pressure (mb). Capital L and $\mathrm{H}$ stand for low and high pressure centre. This map is provided by the Thai Meteorological Department $\ldots . . . . .3$

3.4 Evolution of monthly rainfall $(\mathrm{mm})$ in each climate sub-region in Vietnam. N4 and S1 is the coastal region that has the late rainy season

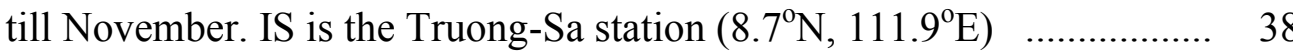

3.5 Thirty two years average (1979-2010) distribution of wind directions in Ha-Noi (48820), Da-Nang (48855), and Ho-Chi-Minh city (\#48900, $10.82^{\circ} \mathrm{N}-106.67^{\circ} \mathrm{E}$ ) represent to North, Central, and South stations. From left to right is the prevailing wind direction in January and July. The calm wind frequencies for those two months are $20.1 \%$ and $17.4 \%$ (Ha-Noi), 44.1\% and 46.1\% (Da-Nang), and 8\% and 10.5\% (Ho-ChiMinh city), respectively

3.6 Track of the tropical Storm PARMA in October 2009. Source: NCHMF, Vietnam

3.7 Breaking historical drought records in Red River on 21 January 2010. The water level was $0.48 \mathrm{~m}$, the lowest in the 100 years of observations. The Long-Bien bridge on that day in Hanoi

3.8 Maximum 24 hours accumulated rainfall 06UTC 02 - 03/11/2009 (left) and the accumulated rainfall in the first four days in 11/2009 (right). Unit: $\mathrm{mm}$

3.9 Surface analysis map on 00zUTC 02/11/2009, MIRINARE-0921 storm. Map is provided by TMD

4.1 Cluster analysis for temperature anomalies (top) and rainfall anomalies (bottom) 1971-2010. From left to right: specification of 6,7,8,9 climate sub-regions, corresponding with $\mathrm{K}=6,7,8,9$ in $\mathrm{K}$-methods. Alphabet letters are assigned to sub-regions

4.2 Variation of observation annual average temperature anomaly (black), JCDAS temperature anomaly averaged (dash) over domain $(8-23 \mathrm{~N}, 102-$ $110 \mathrm{E}$ ), and seven JCDAS gridded points (gray) over Vietnam

4.3 Pearson correlation coefficients between Niño 3.4 index and surface 
temperature in sub-regions in Vietnam. X-axis: month-lag, Y-axis:

Pearson correlation coefficients

4.4 Summer and winter average temperature anomaly $\left({ }^{\circ} \mathrm{C}\right)$. Bold solid lines are the verage anomaly JJA and DJF; thin lines are the average MJJ and NDJ; and dashed lines are the average JAS and JFM temperatures $\ldots . .54$

4.5 First two EOF components of JCDAS rainfall (mm/day) in Annual (top), MJJA (middle), and DJFM (bottom). Percentage variances explained by the leading modes are: Annual (19\%, 8\%), MJJA (13\%, $7 \%)$, DJFM $(12 \%, 8 \%)$

4.6 Correlation maps between annual, DJFM rainfall and the Southern Oscillation Index (SOI). Only correlation coefficients significant at the 90\% level are displayed

4.7 Correlation maps between SSTA and MSLP and annual rainfall (top), MJJA rainfall (middle), and DJFM rainfall (bottom). Only correlation coefficients significant at the $90 \%$ confidence level are displayed .......

4.8 Correlation maps between MSLP in REG1 domain and Vietnam subregion rainfall. From left to right: S1, S2, S3, IS sub-region. From top to bottom: Annual, MJJA, DJFM. Only correlation coefficients significant at the $90 \%$ confidence level are displayed. Contour level: 0.1

5.1 Monthly mean rainfall at some stations around the world. Data for BuonMaThuot and Saigon stations are derived from Bruzon and Carton (1930); other data from the corresponding National Meteorological Services for the period (1960-1990). Unit:mm

5.2 a-b) Heterogeneous correlations of SVD1 of global monsoon rainfall and MSLP(left) and U850(right) using all calendar months, 1979-2010; c-d) Regressions of rainfall (shaded, mm/day), MSLP(left, contours, $\mathrm{Pa}$ ), SSTA(right, contours, $\operatorname{degC}$ ), and $\mathrm{U} 850$ (vectors, $\mathrm{m} / \mathrm{s}$ ) onto the SVD1 proxy variable expansion coefficients (MSLP, left; U850, right); e-f) SVD1 timeseries of rainfall (solid, black) and e) MSLP(left, gray) and f) U850 (right, gray). Y-axes in e-f) are standardized by deviations, X-axis: $1979-2010$ 
5.3 Mean evolution of MSLP (dark gray shaded), U850 (light gray shaded), and the monsoon index NRM (bold black) for 1979-2010 over the REG2. Y-axis: standardized anomaly, X-axis: 5-day moving average from daily datasets

5.4 The same as Figure5.2, but for the year 1989. Red circles from left to right represent crossover points at 15 February, 1 April, 14 April, 17 May, 15 July, and 20 September, respectively

5.5 NRM index (bold solid black), MSLP (dark gray shaded), and U850 (light gray shaded) in 2010 over the REG2 region $\left(7.5-25^{\circ} \mathrm{N}, 100\right.$ $\left.120^{\circ} \mathrm{E}\right)$. Y axis: standardized anomaly; $\mathrm{X}$ axis: daily, 5-day moving average

5.6 Trends of rainfall in Vietnam (left) and Central Vietnam (right) during the monsoon (black) and non-monsoon (gray) period. Trends are detected by the least squared method. AVRI (left) and C (right) denote for the All-Vietnam summer monsoon Rainfall Index (AVRI) and Central Vietnam. OD, WD, 30Nov denote for the monsoon onset day, monsoon withdrawal day, and 30 November. Unit: $\mathrm{mm} /$ day

5.7 Average of monsoon onset-withdrawal phases for 1979-2010 in: a) India Ocean(5-15N, 85-95E), b) North America (30-40N, 110-100W), c) North Africa (5-15N, 35-45E), d) Australia (10S-EQ, 135-145E), e) South America (17.5-7.5S, 52.5-42.5W), f) South Africa (17.5-7.5S, 3545E), g) All-India summer monsoon Rainfall domain, Including extension regions (AIRI) $(5-30 \mathrm{~N}, 65-90 \mathrm{E})$, h) Webster-Yang (1992) index domain (0-20N, 40-110E), i) Goswami et al. (1999) index domain (10-30N, 70-110E), j) Hung and Yanai (2004) index domain (15- 2S, 115-150E), k) Wang and Fan (1999) index domain (5-32.5N, 90140E), and 1) McPhaden et al. (2008) region (30S-30N, 30-120E). MSLP (dark gray shaded), U850 (light gray shaded), rainfall (dotted), Monsoon Index (black). X-axis: daily, 5-day moving average from 01 January, Y-axis: standardized anomalies

6.1 JRA-25 reanalysis streamlines at $925 \mathrm{hPa}$ at 12UTC 26 September 1980. 
Black contours denote the mean sea level pressure, pink shaded areas represent the $24 \mathrm{hr}$ rainfall $(\mathrm{mm})$. The vortex produced $381 \mathrm{~mm} / 24 \mathrm{~h}$ at Dong-Ha station (WMO code \# 48849, triangle dot)

6.2 a) Monthly rainfall of Asean stations from APHRODITE. Used stations: Laos(L(19.98N,102.18E)), Vietnam (V1(18.1N,106.3E), V2(13.8N,109.2E)), Cambodia $\quad(\mathrm{C}(11.56 \mathrm{~N}, 104.9 \mathrm{E})), \quad$ Singapore (S(1.21N,103.54E)), Philippines (P1(16.4N,120.6E), P2(14.5N,121E), P3(9.8N,118.7E)), Malaysia (M1(5.88N,116.19E), M2(3.15N,113.09E), $\mathrm{M} 3(5.28 \mathrm{~N}, 103.1 \mathrm{E})), \quad$ Brunei $\quad(\mathrm{B}(4.58 \mathrm{~N}, 114.36 \mathrm{E})), \quad$ and Indonesia(I1(1.16N,109.14E), I2(3.25S,104.75E), I3(6.27S, 106.9E), I4(6.97S, 110.43N)). Y-axis (mm/day). Core domain of study: (10S-25N, 100-120E). b) Hovmoller diagram of monthly CMAP rainfall over ocean, data being averaged over 100-120E. Only contours greater than $7 \mathrm{~mm}$ /day are displayed. Temporal translocation of rainfall over the region is also illustrated by dotted arrows in a). Unit: $\mathrm{mm} /$ day

6.3 Number appearance of monthly vortices in ocean grid points over the Southeast Asian Sea - Maritime Continent region during 1979-2010.

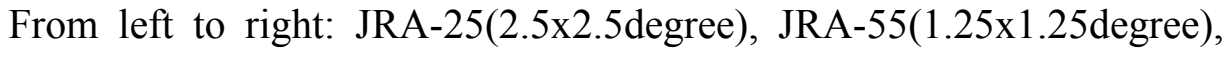
NCEP-DOE (2.5x2.5degree), and ERA-Interim (1.5x1.5 degree) reanalysis dataset, respectively. Contour interval: 20

6.4 a) Monthly occurrence of vortices over sea in the Southeast Asian SeaMaritime continent region in NCEP-DOE reanalysis (1979-2010). Contour lines indicate the frequency of occurrence of vortices in each month. Contour interval: 2

6.4 b) Same as a) except JRA-25 88

6.4 c) Same as a) except JRA-55 89

6.4 d) Same as a) except ERA-Interim 90

6.5 a) Vortex-induced rain days at stations (VS), as a percentage of the total (11680) number of days in the 32 year record (1979-2010). b) same as a) excepts all WMO typhoon-induced rainfall days were removed. Only VS greater than $39 \%$ is displayed 
6.6 Mean temporal distribution of rainfall before (red) and after (black) extracting vortex rainfall in Vietnam (1979-2010). Red and black line represent for observation and vortex-induced rainfall by NCEP-DOE reanalysis dataset, respectively. Vertical lines from left to right denote for the mean onset (day \#130), withdrawal (day \#264), and 30 November (day \#334). Y-axis: mm/day, X-axis: 365 days

6.7 Mean spatial distribution of the difference between before and after extracting vortex-induced rainfall ( $\mathrm{mm} /$ day). Only contours greater than $2 \mathrm{~mm} /$ day are displayed

6.8 Vortex-induced rainfall in summer (a) and post-summer (b) season. Summer and post-summer are identified by the NRM index. Trend is identified by the least squared method. Y-axis: $\mathrm{mm} /$ day. This figure uses ERA-Interim reanalysis data set. 


\section{List of Tables}

Number Page

2.1 List of Vietnam stations used in the study. Data period (19xx-2010), where $\mathrm{xx}$ is the last two digits of the year, varying from 1971 to $2010 \ldots$

3.1 Extreme daily temperatures at some stations (listed in Chapter 2) in Vietnam

3.2 Monthly frequency of tropical cyclones directly impacts to Vietnam (1979-2010)

4.1 Estimated trends in average temperature anomaly in Vietnam in last 40yrs. (unit: ${ }^{\circ} \mathrm{C} / 10$ years). $\mathrm{N} 1, \mathrm{~N} 2, \ldots, \mathrm{VN}$ : climate sub-regions and the whole country. The asterisks $(*)$ indicate the significant level p. $(* * *)$, $(* *),(*),(+): 99.9 \%, 99 \%, 95 \%, 90 \%$ confidence interval for the existence of increased trend, respectively. 90\% $(\mathrm{p}<0.01)$ confidence interval of Sen's slope is in the range estimated by \pm numbers. INS,

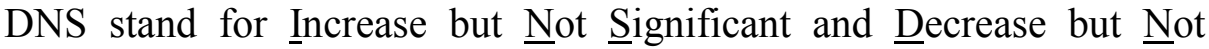
Significant, respectively. Trends for the whole country are calculated by the average of all sub-regions

4.2 Estimated trends in maximum, minimum temperature and rainfall. ( $\mathrm{Tx}$ an. - Tn an.): different temperature between average maximum temperature anomaly ( $\mathrm{Tx}$ an.) and average minimum temperature $\mathrm{Tn}$ anomaly (Tn an.). Dry_ss $=$ Rainfall in Dry Season, Rain_ss $=$ rainfall in Rainy season, and $\sum$ rainfall = total rainfall over 12-month. Other abbreviations see Table 3.1. Unit for changes of rainfall: $\mathrm{mm} / 10 \mathrm{yrs} \quad$......

5.1 Statistics of the leading SVD pairs of global monthly anomalies of rainfall and eight variables (MSLP, U850, Convective rainfall (CONV), 2-m temperature, Out-going long-wave radiation (OLR), zonal wind at $200 \mathrm{hPa}$ (U200), temperature and geopotential height at $850 \mathrm{hPa}(\mathrm{T} 850$, H850)) during 32 years (1979-2010). The first(second)(third) leading mode are separated by parentheses. Covariance and variance unit: \%. The cumulative variance of rainfall accounted for by the leading three 
patterns is about $10 \%$ in each analysis

5.2 Onset dates (OD), Withdrawal dates (WD), and Duration between OD and WD (days) of summer monsoonal rainfall in Vietnam during 19792010. 32-years average and standard deviation of OD, WD, and Duration are described by AVE and STDEV, respectively

5.3 Trends of changes of rainfall in monsoon and post-monsoon period, discriminated by the NRM monsoon index, in each climate sub-region. Trends are identified by the Mann-Kendall test. The signs $\{\uparrow, \downarrow\}$ denote the increase or decrease trend. One arrow means that trend is not significant. Two, three, and four arrows represent for 90\%, 95\% and $99 \%$ confidence interval for the existence of trend. N(1-3), S(1-3), IS, C, and AVRI represent for climate sub-region in northern $(\mathrm{N})$, southern $(\mathrm{S})$, island (IS), Central (C), and Vietnam, respectively. OD, WD, 30Nov denote for the monsoon onset day, monsoon withdrawal day, and 30 November

6.1 Tempo-spatial features on structure and evolution of winter tropical disturbances over the southern Southeast Asian Sea in or closed to the framework of the Winter Monsoon Experiment

6.2 Contribution of vortex rainfall to the total rainfall (\%) during specified periods. Ranges across datasets represent their minimum and maximum values. The original (control) dataset resolutions are $2.5 \times 2.5$ degree for JRA-25 and NCEP-DOE; 1.25x1.25 degree for JRA-55, and 1.5x1.5 degree for ERA-Interim. N1-N4, S1-S3, IS stands for North-to-South climate sub-regions in Vietnam. OD-WD derived from Nguyen et al. (2014b). On average OD-WD covers 125 days, and Post-Summer length is 70 days

6.3 Trends of changes of rainfall in monsoon (OD-WD) and post-monsoon (WD-END) period, discriminated by the NRM monsoon index, in each climate sub-region. Trend is identified by the Mann-Kendall test. The signs $\{\uparrow, \downarrow, \leftrightarrow\}$ denote the increase, decrease, or no trend. One arrow means that trend is not significant. Two, three, and four arrows represent 
for $90 \%, 95 \%$ and $99 \%$ confidence interval for the existence of trend. $\mathrm{N}(1-3), \mathrm{S}(1-3)$, IS, C, and VN represent for climate sub-region in northern (N), southern(S), island (IS), Central (C), and Vietnam, respectively. OD, WD, END denote for Onset day, Withdrawal day, and 30 November 


\section{CHAPTER 1}

\section{INTRODUCTION}

In all things of nature there is something of the marvelous.

Aristotle 


\subsection{Climatology, interannual variations, and trends}

Vietnam is a Southeast Asian country, lying between $8.30^{\circ} \mathrm{N}$ and $23.22^{\circ} \mathrm{N}$, and has a long coast line, approximately $4000 \mathrm{kms}$. Vietnam has a typical tropical monsoon with a

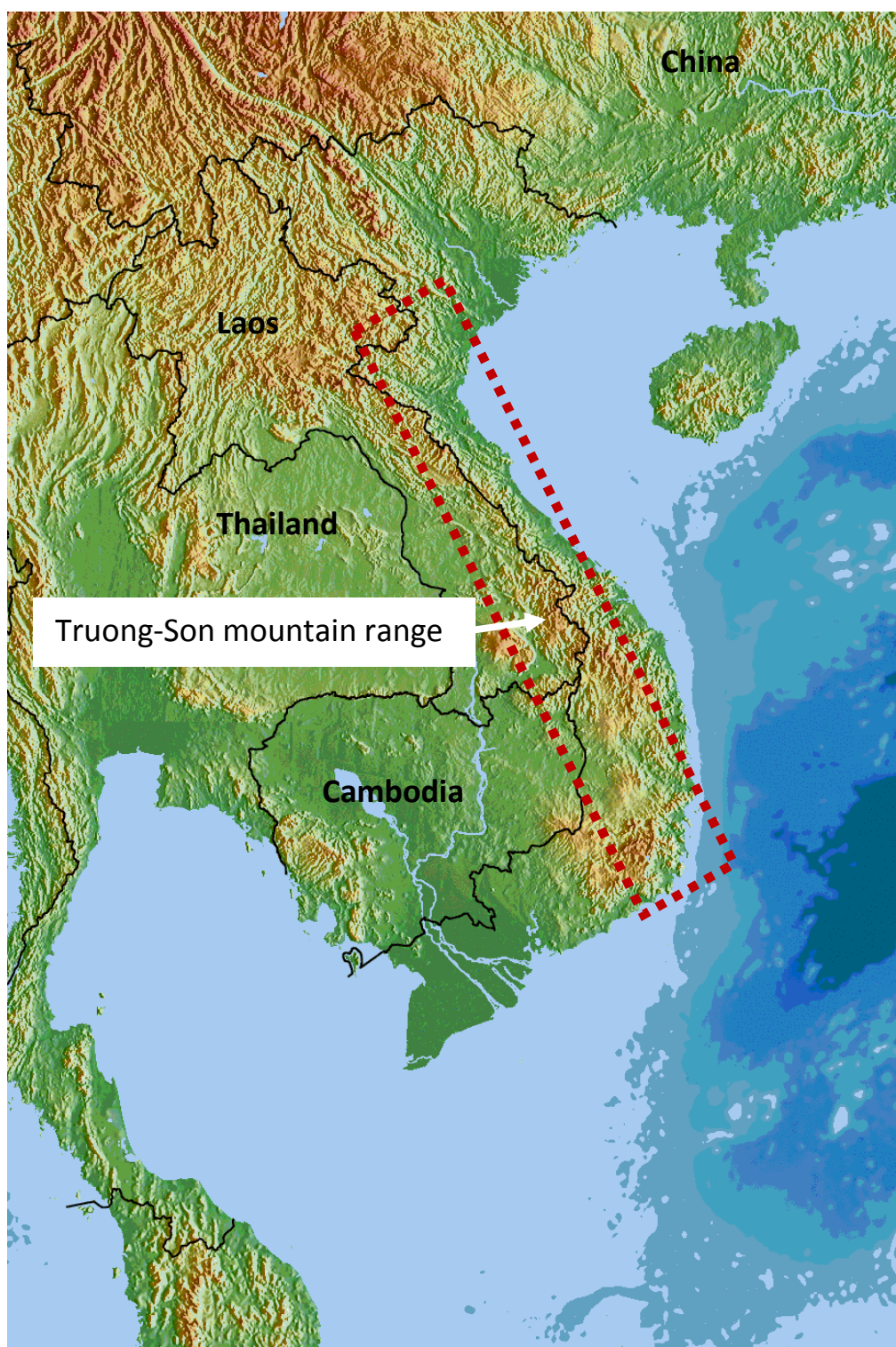

Figure 1.1 Natural geographical map of Vietnam. Rivers (mountains) are represented by light green (dark yellow). The darker the blue, the deeper the sea floors over the Southeast Asian Sea.The red dot-box shows the Truong-Son range, the longest mountain in Vietnam. seasonal reversal in atmospheric circulation and precipitation associated with the thermal contrast in eastwest or land-sea heating. Mountains above $1000 \mathrm{~m}$ are found in the Northwest, Central, and Central Highland regions. TruongSon mountain range, on average $600-1000 \mathrm{~m}$ in height and about $100 \mathrm{~km}$ wide separating Vietnam and Laos, is a topography feature in Central Vietnam (Figure 1.1). In summer (winter), the southwesterly (northeasterly) prevails (Figure 1.2).

Sub-regional climate rainfall is characterised by monsoons and their interaction with the orography. On average, in the rainy season, the total

amount of monthly rainfall at any station can vary from $200-300 \mathrm{~mm}$ to $500-600 \mathrm{~mm}$ (more detail in Chapter 3). 

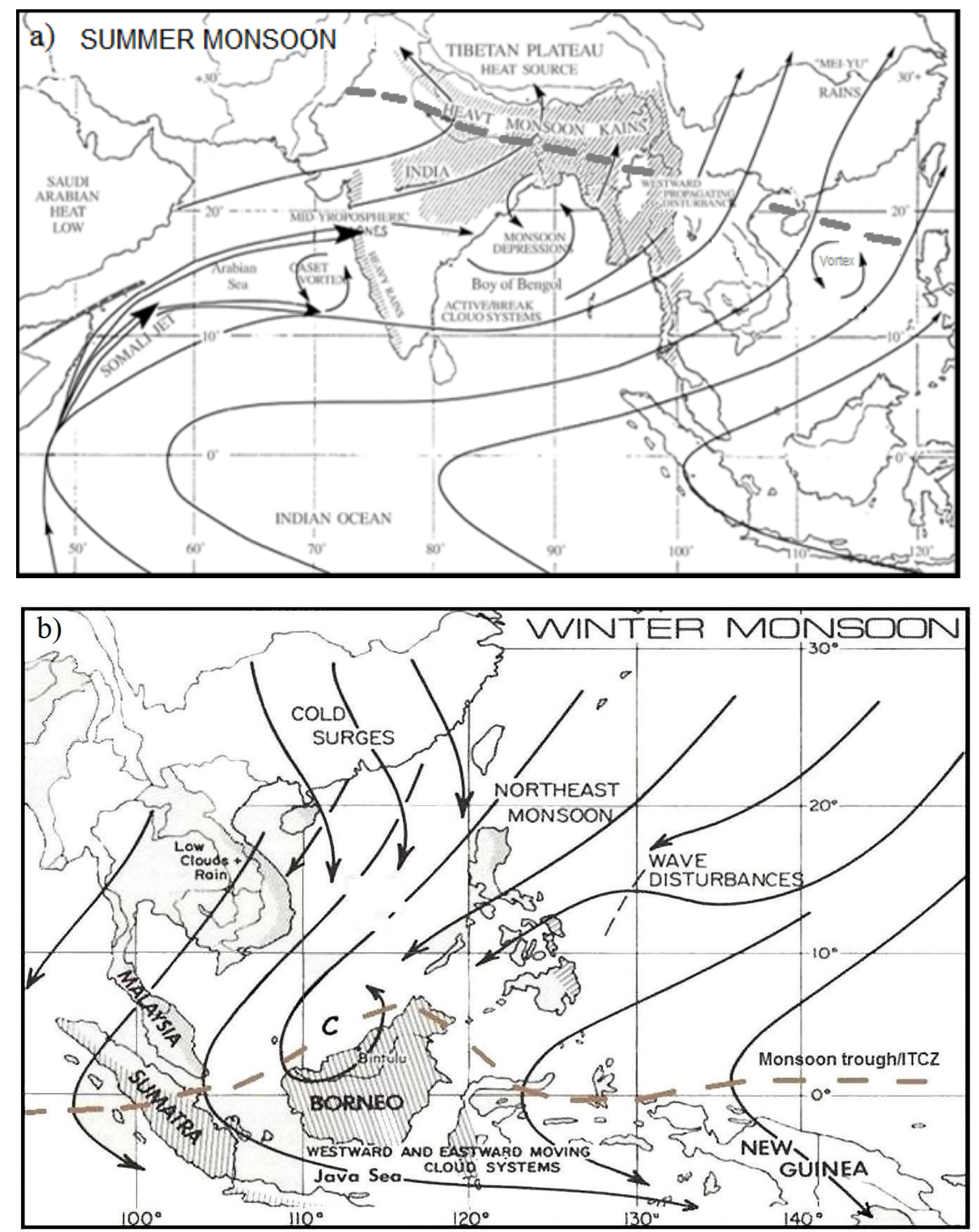

Figure 1.2 a) Lead surface synoptic-scale weather systems in summer in the Southeast Asian - Maritime Continent (SEAMC) region. Monsoon trough/ITCZ is displayed by the bold-dash line (Ding and Sikka, 2006). b) Same as a), except for winter (Johnson and Houze, 1987). 
Studies show that during the southwesterly season, heavy rainfall centres appear in the North and South of Vietnam (Bruzon and Carton, 1930; Ramage, 1952;Chen et al., 2004; Yen et al., 2011). The reason is that, from May to September, when the southwesterly prevails, most of the central coastal stations are in the rain shadow of the Truong-Son mountain ridge. The Foehn effect, results in dry and hot westerlies in the lee of the Truong-Son ridge, and raises the average temperature on the central coast of Vietnam.

From September/October to December, when the prevailing winds are northeasterly, central coastal stations are on the windward side of the Truong-Son mountains and thus increased rainfall amounts are recorded in this region (Figure 1.2b). This was known from studies such as Nguyen and Nguyen (2004), Yokoi and Matsumoto (2008), and Yen et al. (2011).

Mid-December to April is the dry season, monthly rainfall is usually less than $100 \mathrm{~mm}$ (Figure 1.3). During this time, the prevailing winds are northeasterlies.

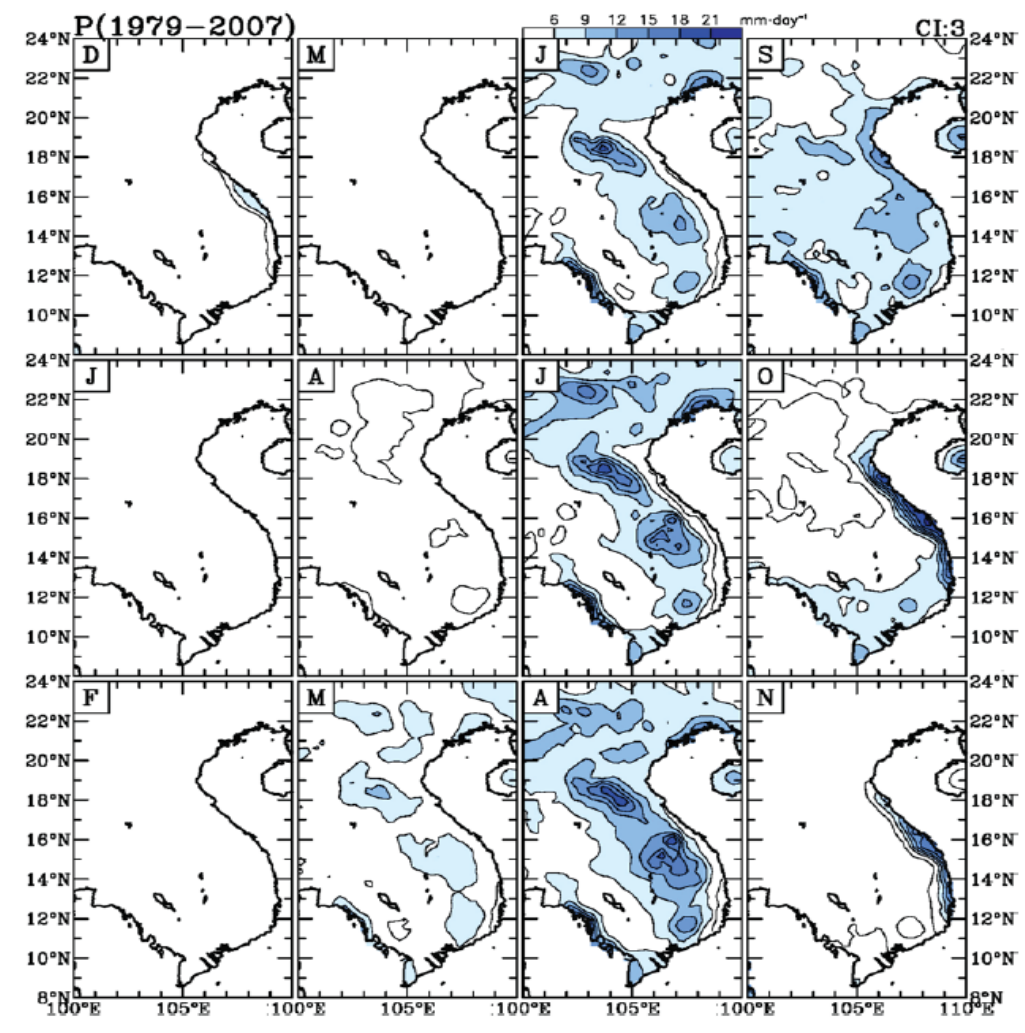

Figure 1.3 Monthly mean climatology of Vietnam rainfall (averaged 1979-2007). Contour interval: 3mm.day ${ }^{1}$ (Yen et al., 2011) 
One question may be raised: "With the same northeasterlies prevailing from September this year to April in the following year, what mechanism(s) can produce huge amount of rainfall during some first months, but, totally changed to, dry in the later months?". One possibility is the involvement of tropical vortices (more discussions in the next Section 1.3, Chapter 3, and Chapter 6).

Apart from prevailing northeasterlies, cold surges also occur during the winter time in Vietnam (Figure 1.2b). Crossing Viet Nam, cold surges are accompanied by squall lines, gales, showers, and a sharp drop temperature. A major cold surge may drop the average daily surface temperature by at least $3-5^{\circ} \mathrm{C}$ in two consecutive days and may persist for 3-5 days on average before withdrawal in near equatorial locations (NCHMF, 2009). A surge itself produces little rainfall (showers), however, when it interacts with tropical vortices, it can produce heavy rainfall events (Chang et al. 2005; Yokoi and Matsumoto, 2008; Chen et al., 2012). The record-breaking $977 \mathrm{~mm}$ on 03/11/1999, observed in Hue - a Central station, was the result of the interaction between a cold surge and a tropical vortex (detail in Chapter 3).

The interannual variability of climate in Vietnam is the next interest in this study. In the wider region, SEAMC rainfall is correlated to the ENSO activity (Meehl, 1987; Lau and Yang, 1997; Wang, 2006). More specifically, in warm events, the normal west-to-east circulation between the Indian and Pacific oceans is weakened and anomalous heavy convection and high sea surface temperatures (SST) in the tropical Pacific tend to be associated with suppressed convection in the monsoons over southern Asia. Decreased rainfall in the Indian, Southeast Asian or Australian monsoon region are observed. In cold events, the reverse patterns occur; meaning rainfall in those mentioned regions is increased. In Vietnam, ENSO signals were first found in rainfall time-series in Central. For example, Nguyen et al. (2007) showed month-to-month relationships during the rainy season between the precipitation in the Central Highland and SST over the tropical Pacific and Indian Ocean. These authors showed that, except for May, the rainfall in the Central Highland has a relationship either with SST in the tropical Pacific or the Indian Ocean. Yen et al. (2011) investigated the Central rainfall variation during October-November, and showed that its interannual variability is strongly related to ENSO $(\mathrm{r}=-0.7)$. 
With the greater interest in global warming, studies on climate trends have massively increased in recent decades. In the Southeast Asian region, the first major study which examined trends in extreme events in six countries during 1961-1998 was carried out by Manton et al. (2001). Due to the lack of publicly-accessible observations, a limited number of stations were used; including only one from Vietnam. While most stations exhibited statistically significant increases in the frequency of hot extremes, and decreases in cold extremes, the extreme rainfall indices showed less spatial consistency. More specifically, the Vietnamese station showed "no significant trend in any of the rainfall indices, although there were decreases in all indices except the percent of total rainfall from extreme events (which showed no trend)". For the same purpose, Endo et al. (2009) and Caesar et al. (2011) examined the trend of climate extremes from different observation datasets in different periods of time. Endo et al. (2009) selected 203 Southeast Asian stations (58 Vietnamese stations) during 1950s-2000s, while Caesar et al. (2011) used 67 Indo-Pacific stations (13 Vietnamese stations) in 1971-2005. The former found that heavy precipitation increases (decreases) in southern (northern) Vietnam; the latter showed that warm (cold) extremes are increasing (decreasing), even though trends in precipitation extremes are not spatially coherent.

Thus, as seen here and there, climate characteristics in all sub-regions are not fully described. Here we extend the former dataset (1971-2000), by a further 10 years (20012010) and use a more complete, comprehensive analysis in this first part of our study. We will not be limited to a single station or a shortened period as were the aforementioned studies. Here rainfall variation, rainfall and ENSO relationship, and trend of changes in each sub-region will be examined for all stations (Chapter 3, Chapter 4).

\subsection{Monsoon variability}

Seasonal variability of the Asian monsoon is described through summer monsoon onset studies (Tao and Chen, 1987; Lau and Yang, 1997). These authors identified that the mean onset in the Southeast Asian region is around mid-May and being the earliest onset stage of the entire Asian summer monsoon system, and the year-to-year onset variation in this region is large. During a monsoon season there is observed "active/break" episodes, and the 
lead oscillation mode of an active episode is 30-60 days (Lau and Yang, 1997; Yokoi et al., 2007).

Along with monsoon variability studies, dominant atmospheric circulation pattern associated with the onset of the Southeast Asian monsoon is documented. The Western Pacific subtropical High (WPSH) and South Asian High (SAH) activity are considered to be control mechanisms of monsoon onset in the region (Lau and Yang, 1997; Kue and Lin, 2010). Other different atmospheric elements involved in the onset process, include, but are not limited to, meridional temperature gradient in the upper troposphere (Mao and Chan, 2004), and a combination of moist static energy, lower winds, Mean Sea Level Pressure, and Outgoing Long-wave Radiation (Quian and Lee, 2000) and (Pham et al., 2010). Wang et al. (2008) listed twenty-five different indices to examine the summer monsoon onset.

The twenty-five different monsoon onset indices mentioned in Wang et al. (2008), on the one hand, showed the complexity of relationships associated with monsoon onset dates (OD); on the other hand they suggested that there is more than one solution for the OD identification. Since only few studies raised the important role of monsoon withdrawal dates (WD), which is associated with OD to identify the summer monsoon length, a new index to identify both OD and WD is expected. Here we introduce a new unified index to measure the length of the monsoon period. We test the suitability of this index for use in other monsoon regions and use it here to study the interannual variations of monsoon rainfall in Vietnam (Chapter 5).

\subsection{Tropical cyclones activity and their associated rainfall}

Rainfall peaks in the North and South occur in summer, however in Central maximum rainfall occurs in late autumn (Section 1.1). Though the total amount of rainfall in a rainy season is comparable in each sub-region, Central witnesses more frequent severe floods than other regions. For example, the record-breaking $977 \mathrm{~mm}$ on 3 November 1999 was observed in Hue, a Central station. This extreme weather event caused deaths and property damages. Therefore it is understandable that previous studies focused on the mechanism of Central precipitation. 
Yen et al. (2011) found the northwest-southeast Truong-Son mountain range can be the prime factor to producing the regional precipitation. In general, in winter time, the northeasterly water vapour flux from ocean toward Vietnam interacts and is almost perpendicular to the Truong-Son mountain range. This interaction sets an environment favorable for the orographically-induced rainfall.

Along with orography, tropical cyclone activity is also a major factor in producing significant rainfall in Central. Vietnam is located in one of the most severe tropical cyclone basins in the world, the territory often suffers from the direct impact of tropical cyclones from the Western Pacific and/or from the Southeast Asian Sea (SEAS) region (Figure 1.4). Crossing the Philippines, then SEAS, these tropical cyclones hit the coastal areas of Vietnam, causing heavy rainfall, flood, and inundation. Early climate studies in Vietnam showed a higher frequency of cyclone activity from summer (June-July-August) to late autumn (October-November) along the coast from North to Central, and South (e.g: Bruzon and Carton, 1930).

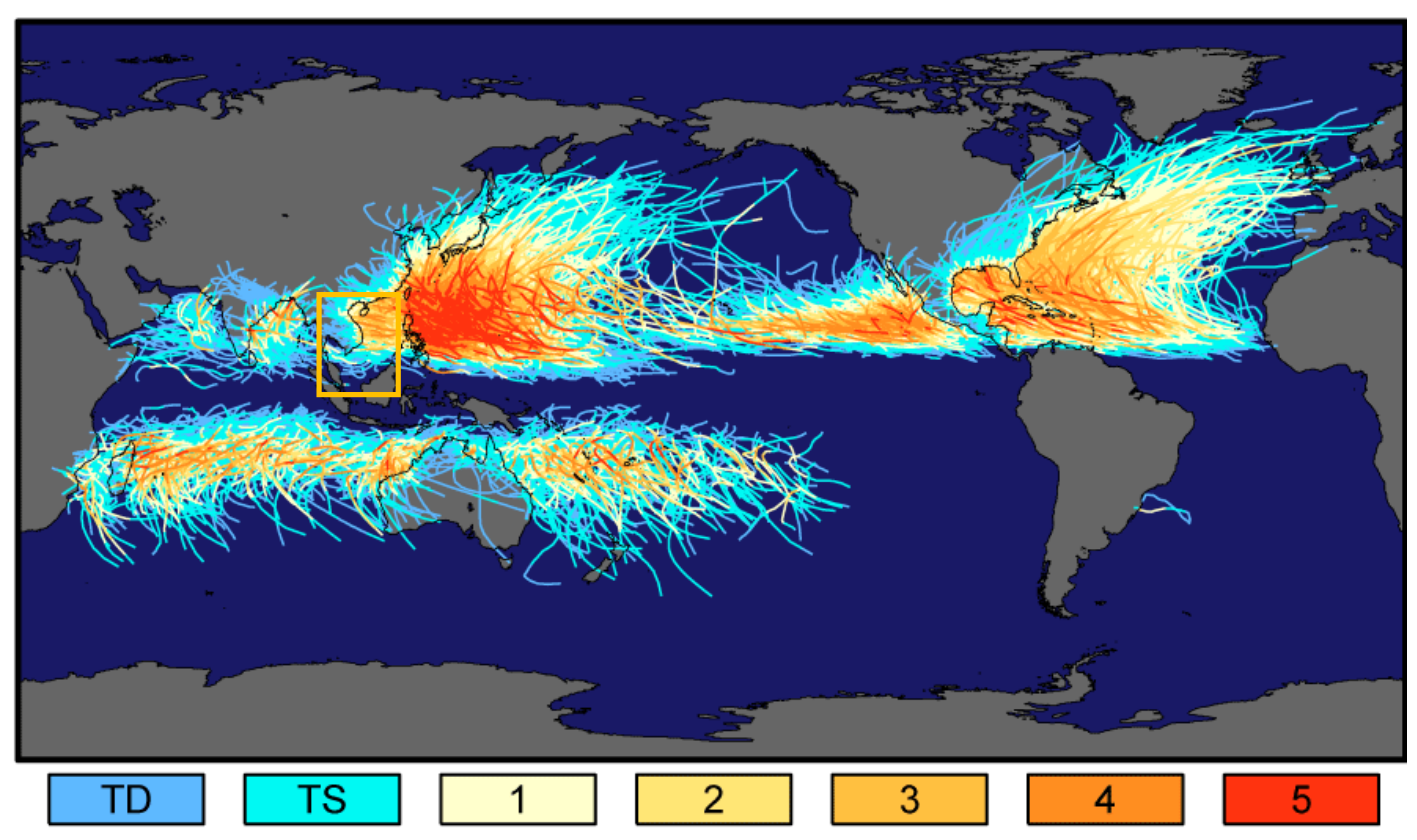

Figure 1.4 Track and intensity of all tropical storms, 1851-2006. The diagram uses the Saffir Simpson hurricane intensity scale. Vietnam is highlighted by the yellow box. (Source: http://www.goesr.gov/education/comet/satmet/remote_sensing/navmenu.php_tab_2_page_1.2.0_type_text.htm). 
These studies also showed the co-existence of the rainfall peak in Central Vietnam (through the station observation records) and the activity of tropical cyclones (through maps of tropical cyclones tracks) in late summer.

In the larger tropical Pacific and Indian Ocean region, there is a heavy convective rainfall centre moving from north to south and west to east from summer to winter, and its return excursion from summer to winter. This annual cycle is associated with Intertropical Convergence Zone (ITCZ) and South Pacific Convergence Zone (SPCZ) activity (Meehl, 1987; Chang et al., 2005).

Here we 1) investigate the Central precipitation in regard to tropical cyclone contributions; and 2) explore the incursion of tropical cyclones over the whole SEAMC region (detail in Chapter 6).

\subsection{Objectives and Outcomes}

The first part of this Ph.D project examines climate variability and climate trends over Vietnam using a more complete set of in-situ data than used in previous studies. Sixty stations distributed throughout all sub-regions and an extended data period (1971- 2010), are employed.

This has enabled the most comprehensive study of temperature and rainfall trends for all Vietnam. Results show increasing temperature trends are statistically significant in all regions of the country at both seasonal and annual time-scales. However rainfall trends are not statistically significant in six of the eight climate sub-regions.

In the framework of global warming, the trend of average temperature anomalies in Vietnam is comparable with those globally (e.g: Jones et al., 2007; IPCC, 2013), however the rainfall variability identified in the first part of the study needs to be examined in more detail. This is the focus of the next part of my study.

In the second part, we look closer at the variability of rainfall. More insights can be gained with the use of a daily dataset. Many previous studies attempted to determine the summer monsoon onset as a part of the bigger picture on seasonal rainfall variability. However, only few studies examined the length of the monsoon season i.e. onset- 
withdrawal (OD-WD) which is key factor in the study of the interannual variations of rainfall.

Here we establish an index which can detect both OD and WD. Ideally a monsoon index should include consideration of the seasonal change of rainfall and associated changes in prevailing winds. Since rainfall is intermittent in space and time, we seek proxies that can characterize rainfall in terms of smoothly varying large-scale parameters. Among the most commonly used rainfall proxies (detailed in Chapter 3), mean sea level pressure (MSLP) is often chosen as an index component because it shows strong relationships with rainfall (e.g., Walker and Bliss, 1932; Meehl, 1987), and it varies more smoothly and exhibits larger spatial scale compared to rainfall. The second index component is low-level winds, representing the dynamic features of monsoon circulation. To diminish orographic effects, winds at $850 \mathrm{hPa}$ are selected.

One of the successes in this second part of the study is the NRM ${ }^{1}$ monsoon index NRM which combines regional mean sea level pressure and zonal wind at $850 \mathrm{hPa}$. While originally developed to define onset and withdrawal dates for the monsoon in Vietnam and over Southeast Asia, the index is also shown to be applicable in all monsoon regions of the globe, the first objective index to have such global utility.

The third part of this project develops from the first two parts. The monsoon index identified the mean OD-WD in Vietnam is 10 May-21 September on average. In the first part we know that the rainy season in Central Vietnam peaks in September-OctoberNovember. Therefore there exists a period, about two months, mostly October-November, of quite significant rainfall has not received much attention in the scientific literature. Here we are interested in the contribution of tropical vortex activity to regional rainfall.

We demonstrate the existence of a semi-permanent vortex near the south coast of Vietnam. It is this vortex and tropical cyclones over the Southeast Asian Sea-Maritime Continent (SEASMC) that contribute significantly to precipitation, particularly the postmonsoon rainfall in Central Vietnam.

\footnotetext{
${ }^{1}$ NRM after the authors Nguyen, Renwick, and McGregor.
} 
The thesis contains seven chapters. This introduction chapter (Chapter 1) is followed by the data and methodology in Chapter 2. Chapter 3 and 4 are a description of the climatological variation of temperature and rainfall in Vietnam. Chapter 5 presents in detail the establishment of the monsoon index and its application. Vortex activity and its associated rainfall are investigated and described in Chapter 6. The last chapter is for conclusions and discussions. 


\section{CHAPTER 2}

\section{DATA AND METHODOLOGY}

In God we trust, all others bring data.

\section{William Edwards Deming}

This chapter is a comprehensive summary of data sets and methodologies used throughout the thesis. Descriptions of the data sets and study domains are given, along with an introduction of statistical analysis techniques and the links between them. This chapter provides the basis for the study goals on climate variability, climate trends, monsoon and tropical vortex activity and their associated rainfall in the region. 


\subsection{Study domain}

Several domains are used in this study (Figure 2.1). The climate and weather regimes in the SEAMC region are mostly dominated by the atmospheric circulations of this vast region (Wang, 2006). To demonstrate the general climatology of the SEAMC in a wider setting, the region of $50^{\circ} \mathrm{S}-70^{\circ} \mathrm{N}$ and $40^{\circ} \mathrm{E}-120^{\circ} \mathrm{W}$, hereafter REG1, was chosen. This domain ensures that synoptic scale information from most of Pacific Ocean, Indian Ocean, Tibetan plateau, Lake Baikal, and Asia-Australia region is represented.

For the studies of the monsoon onset-withdrawal phases and SEAMC rainfall, smaller domains REG2 and REG3 are chosen, $\left(7.5-25^{\circ} \mathrm{N}, 100-120^{\circ} \mathrm{E}\right)$ and $\left(10^{\circ} \mathrm{S}-25^{\circ} \mathrm{N}, 100-\right.$ $120^{\circ} \mathrm{E}$ ), respectively. The selection of REG2 domain is based on the fact that the Asian monsoon onset is first observed over the Indochina peninsula in May, then the summer monsoon rain band moves northwards up to China-Japan-Korea in following months (Wang and LinHo, 2002). It remains stationary in northern parts of East Asia for one to two months then starts to move backward to lower latitudes, and is associated with peak rainfall over Indochina-Vietnam, Philippines, and lastly Singapore, Malaysia, and Indonesia in northern winter time. This rain-band migration feature is investigated using the REG3 domain $\left(10^{\circ} \mathrm{S}-25^{\circ} \mathrm{N}, 100^{\circ} \mathrm{E}-120^{\circ} \mathrm{E}\right)$. The Asian monsoon studies were extended to the global tropical monsoon, over the region between $40^{\circ} \mathrm{S}$ and $40^{\circ} \mathrm{N}$, as illustrated in Figure 2.1. 


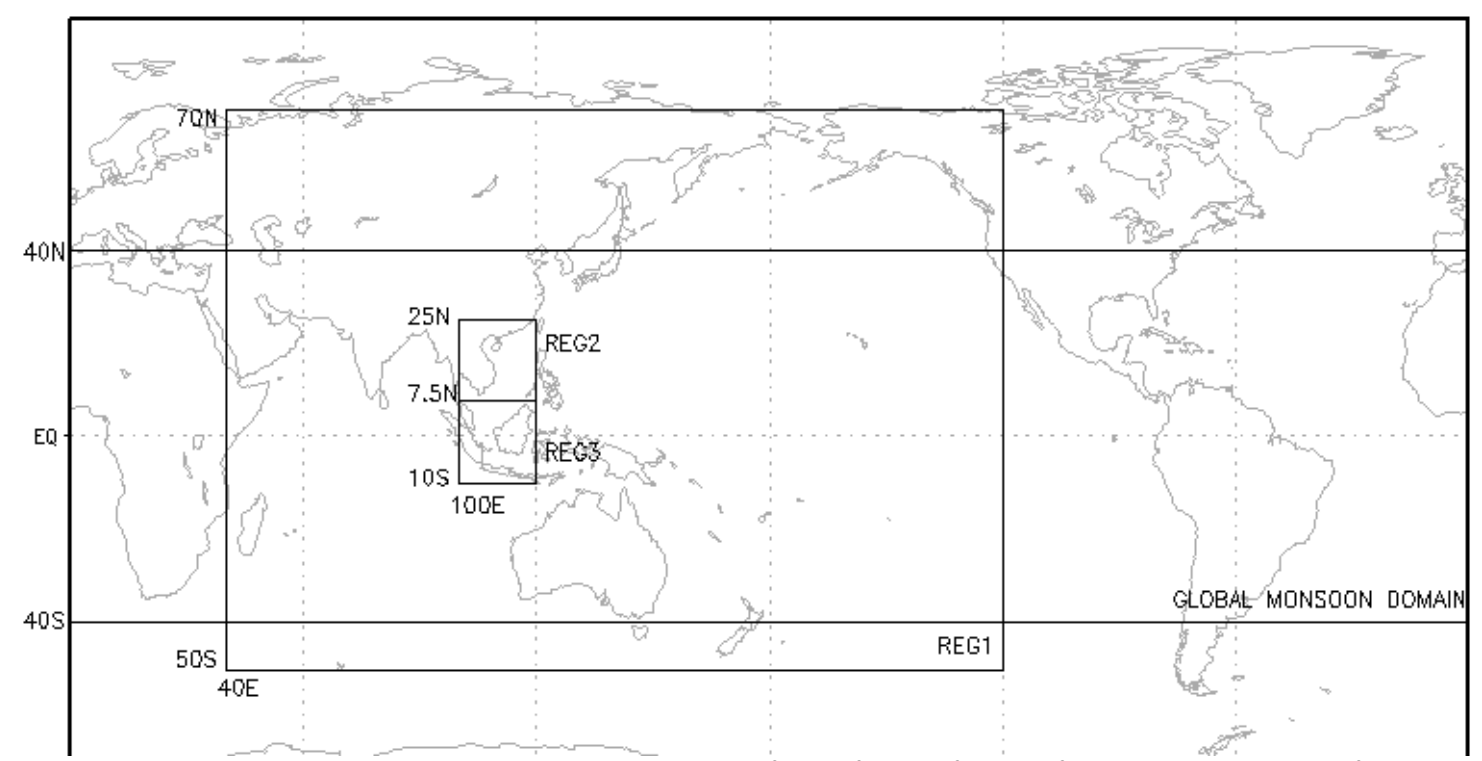

Figure 2.1. Domains used in the study. REG1 $\left(50^{\circ} \mathrm{S}-70^{\circ} \mathrm{N} ; 40^{\circ} \mathrm{E}-120^{\circ} \mathrm{W}\right), \mathrm{REG} 2\left(7.5-25^{\circ} \mathrm{N} ; 100-\right.$ $\left.120^{\circ} \mathrm{E}\right)$, REG3 $\left(10^{\circ} \mathrm{S}-25^{\circ} \mathrm{N} ; 100-120^{\circ} \mathrm{E}\right)$, and Global Tropical Monsoon $\left(40^{\circ} \mathrm{S}-40^{\circ} \mathrm{N} ; 0-360^{\circ}\right)$. 


\subsection{Data}

The studies discussed here use data from a variety of different sources. They include dynamic atmospheric reanalysis, satellite-derived rainfall, station observations, and typhoon tracks. Here we describe the origin, characteristics and use of these data. A further description can also be found in Nguyen et al. (2014a,b).

\subsubsection{In-situ observations}

Page et al. (2004) noted, through a World Climate Data and Monitoring Programme Report, that the Hydrometeorological Data Centre in Vietnam stores paper records in more than thirty rooms. A digitization programme of archived data began in 2001 through the WMO Voluntary Cooperation Programme, although only the data collected since the 1960s have been stored in digital format. During the war years, 1945-1954, and in the 1970s, observations at several stations were not made. In this Ph.D project, we selected a list of stations that are least affected by missing data.

For case studies of monsoon characteristics and SEASMC vortex rainfall, daily and monthly temperatures at the 2-metre level and rainfall from sixty climate stations in Vietnam for the period 1971-2010 are presented. These stations are distributed throughout all sub-regions of Vietnam (Figure 2.2).

Station locations have not changed since the 1970s, nor have the instruments used to record temperature and rainfall changed during the study period. The observational datasets were provided by the Vietnam National Centre for Hydrometeorological Forecasting. In our study all data records for northern stations were collected from the year 1971, while temperatures in the South Vietnam were mostly recorded from 1976, one year after the end of the Vietnam War. Climate normals are defined by the period $(197 x-2000)$ where $\mathrm{x}$ is the first observation year in Table 2.1. The most recent decade, 2001-2010, is used to compare with statistics from the normal period. In this study the Central Vietnam, a region consists of the $\mathrm{N} 4$ and $\mathrm{S} 1$ sub-regions, is usually to be referred as the Central; the North means N1-N4 sub-regions, and the South means S1-S3 sub-regions. 


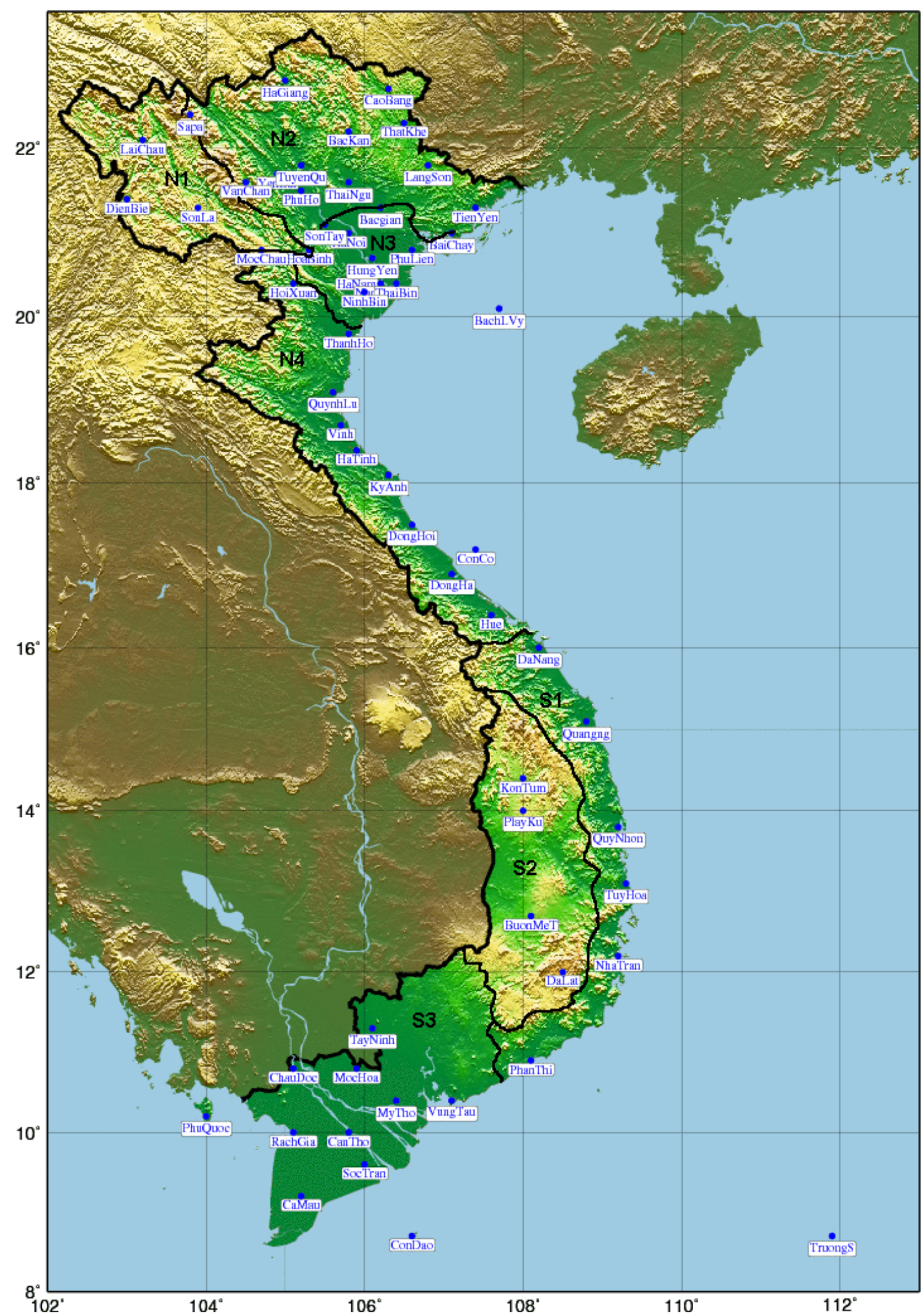

Figure 2.2. Sixty Vietnamese observation stations used in the study. N1, N2, N3, N4 (S1, S2, S3) stands for Northern

(Southern) climate sub-regions. Central region is considered to be the combination of N4 and S1 sub-regions. Truong-Sa $\left(8.7^{\circ} \mathrm{N}, 111.9^{\circ} \mathrm{E}\right)$ is the farthest offshore station. (See Table 2.1 for station details) 
Station details are listed in Table 2.1.

\begin{tabular}{|c|c|c|c|c|c|c|c|c|}
\hline \multirow[t]{2}{*}{$\mathrm{N}^{\mathrm{O}}$} & \multirow{2}{*}{$\begin{array}{l}\text { Station } \\
\text { name }\end{array}$} & \multirow{2}{*}{$\begin{array}{l}\text { Station } \\
\text { code }\end{array}$} & \multirow{2}{*}{$\begin{array}{l}\text { Latitude } \\
\left({ }^{\circ} \mathrm{N}\right)\end{array}$} & \multirow{2}{*}{$\begin{array}{l}\text { Longitude } \\
\left({ }^{\circ} \mathrm{E}\right)\end{array}$} & \multirow{2}{*}{$\begin{array}{l}\text { Altitude } \\
\text { (m) }\end{array}$} & \multicolumn{2}{|c|}{ Monthly (19xx-2010) } & \multirow{2}{*}{$\begin{array}{l}\text { Daily } \\
\text { Rainfall }\end{array}$} \\
\hline & & & & & & Temp. & Rainfall & \\
\hline \multicolumn{9}{|c|}{ Northwest climate region (N1) } \\
\hline 1 & LaiChau & 48800 & 22.1 & 103.2 & 243 & $71-10$ & $71-10$ & $79-10$ \\
\hline 2 & DienBie & 48811 & 21.4 & 103 & 475 & $71-10$ & $71-10$ & $79-10$ \\
\hline 3 & SonLa & 48806 & 21.3 & 103.9 & 675 & $71-10$ & $71-10$ & $79-10$ \\
\hline 4 & MocChau & $48 / 25$ & 20.8 & 104.7 & 972 & $71-10$ & $71-10$ & $79-10$ \\
\hline \multicolumn{9}{|c|}{ Northeast climate region (N2) } \\
\hline 5 & HoaBinh & 48818 & 20.8 & 105.3 & 23 & $71-10$ & $71-10$ & $79-10$ \\
\hline 6 & Sapa & 48802 & 22.4 & 103.8 & 1584 & $71-10$ & $71-10$ & $79-10$ \\
\hline 7 & YenBai & 48815 & 21.7 & 104.9 & 56 & $71-10$ & $71-10$ & $79-10$ \\
\hline 8 & VanChan & $48 / 14$ & 21.6 & 104.5 & 275 & $71-10$ & $71-10$ & $79-10$ \\
\hline 9 & HaGiang & 48805 & 22.8 & 105 & 117 & $71-10$ & $71-10$ & $79-10$ \\
\hline 10 & TuyenQu & 48812 & 21.8 & 105.2 & 41 & $71-10$ & $71-10$ & $79-10$ \\
\hline 11 & BacKan & 48810 & 22.2 & 105.8 & 174 & $71-10$ & $71-10$ & $79-10$ \\
\hline 12 & ThaiNgu & 48831 & 21.6 & 105.8 & 35 & $71-10$ & $71-10$ & $79-10$ \\
\hline 13 & PhuHo & $48 / 51$ & 21.5 & 105.2 & 54 & $71-10$ & $71-10$ & $79-10$ \\
\hline 14 & CaoBang & 48808 & 22.7 & 106.3 & 244 & $71-10$ & $71-10$ & $79-10$ \\
\hline 15 & LangSon & 48830 & 21.8 & 106.8 & 258 & $71-10$ & $71-10$ & $79-10$ \\
\hline 16 & ThatKhe & 48807 & 22.3 & 106.5 & 162 & $71-10$ & $71-10$ & $79-10$ \\
\hline 17 & BaiChay & 48833 & 21 & 107.1 & 38 & $71-10$ & $71-10$ & $79-10$ \\
\hline 18 & TienYen & 48837 & 21.3 & 107.4 & 14 & $71-10$ & $71-10$ & $79-10$ \\
\hline
\end{tabular}




\begin{tabular}{|c|c|c|c|c|c|c|c|c|}
\hline \multirow[t]{2}{*}{$\mathrm{N}^{\mathrm{o}}$} & \multirow{2}{*}{$\begin{array}{l}\text { Station } \\
\text { name }\end{array}$} & \multirow{2}{*}{$\begin{array}{l}\text { Station } \\
\text { code }\end{array}$} & \multirow{2}{*}{$\begin{array}{l}\text { Latitude } \\
\left({ }^{\circ} \mathrm{N}\right)\end{array}$} & \multirow{2}{*}{$\begin{array}{l}\text { Longitude } \\
\left({ }^{\circ} \mathrm{E}\right)\end{array}$} & \multirow{2}{*}{$\begin{array}{l}\text { Altitude } \\
\text { (m) }\end{array}$} & \multicolumn{2}{|c|}{ Monthly (19xx-2010) } & \multirow{2}{*}{$\begin{array}{l}\text { Daily } \\
\text { Rainfall }\end{array}$} \\
\hline & & & & & & Temp. & Rainfall & \\
\hline \multicolumn{9}{|c|}{ Red River Delta climate region (N3) } \\
\hline 19 & Bacgian & 48809 & 21.3 & 106.2 & 8 & $71-10$ & $71-10$ & $79-10$ \\
\hline 20 & PhuLien & 48826 & 20.8 & 106.6 & 112 & $71-10$ & $71-10$ & $79-10$ \\
\hline 21 & HaNoi & 48820 & 21 & 105.8 & 6 & $71-10$ & $71-10$ & $79-10$ \\
\hline 22 & SonTay & 48817 & 21.1 & 105.5 & 16 & $71-10$ & $71-10$ & $79-10$ \\
\hline 23 & HungYen & 48822 & 20.7 & 106.1 & 3 & $71-10$ & $71-10$ & $79-10$ \\
\hline 24 & NamDinh & 48823 & 20.4 & 106.2 & 2 & $71-10$ & $71-10$ & $79-10$ \\
\hline 25 & HaNam & 48821 & 20.5 & 105.9 & 3 & $71-10$ & $71-10$ & $79-10$ \\
\hline 26 & NinhBin & 48824 & 20.3 & 106 & 3 & $71-10$ & $71-10$ & $79-10$ \\
\hline 27 & ThaiBin & 48835 & 20.4 & 106.4 & 2 & $71-10$ & $71-10$ & $79-10$ \\
\hline 28 & BachLVy & 48839 & 20.1 & 107.7 & 56 & $71-10$ & $71-10$ & $79-10$ \\
\hline \multicolumn{9}{|c|}{ North Central climate region (N4) } \\
\hline 29 & ThanhHo & 48840 & 19.8 & 105.8 & 4 & $71-10$ & $71-10$ & $79-10$ \\
\hline 30 & Vinh & 48845 & 18.7 & 105.7 & 5 & $71-10$ & $71-10$ & $79-10$ \\
\hline 31 & QuynhLu & $48 / 77$ & 19.1 & 105.6 & 2 & $71-10$ & $71-10$ & $79-10$ \\
\hline 32 & HoiXuan & 48842 & 20.4 & 105.1 & 102 & $71-10$ & $71-10$ & $79-10$ \\
\hline 33 & HaTinh & 48846 & 18.4 & 105.9 & 3 & $71-10$ & $71-10$ & $79-10$ \\
\hline 34 & KyAnh & $48 / 86$ & 18.1 & 106.3 & 3 & $71-10$ & $71-10$ & $79-10$ \\
\hline 35 & DongHoi & 48848 & 17.5 & 106.6 & 6 & $76-10$ & $71-10$ & $79-10$ \\
\hline 36 & DongHa & 48849 & 16.9 & 107.1 & 8 & $76-10$ & $71-10$ & $79-10$ \\
\hline 37 & ConCo & $48 / 89$ & 17.2 & 107.4 & 3 & $76-10$ & $71-10$ & $79-10$ \\
\hline 38 & Hue & 48852 & 16.4 & 107.6 & 10 & $76-10$ & $71-10$ & $79-10$ \\
\hline
\end{tabular}




\begin{tabular}{|c|c|c|c|c|c|c|c|c|}
\hline \multirow[t]{2}{*}{$\mathrm{N}^{0}$} & \multirow{2}{*}{$\begin{array}{l}\text { Station } \\
\text { name }\end{array}$} & \multirow{2}{*}{$\begin{array}{l}\text { Station } \\
\text { code }\end{array}$} & \multirow{2}{*}{$\begin{array}{l}\text { Latitude } \\
\left({ }^{\circ} \mathrm{N}\right)\end{array}$} & \multirow{2}{*}{$\begin{array}{l}\text { Longitude } \\
\left({ }^{\circ} \mathrm{E}\right)\end{array}$} & \multirow{2}{*}{$\begin{array}{l}\text { Altitude } \\
\text { (m) }\end{array}$} & \multicolumn{2}{|c|}{ Monthly (19xx-2010) } & \multirow{2}{*}{$\begin{array}{l}\text { Daily } \\
\text { Rainfall }\end{array}$} \\
\hline & & & & & & Temp. & Rainfall & \\
\hline \multicolumn{9}{|c|}{ South Central climate region (S1) } \\
\hline 39 & DaNang & 48855 & 16 & 108.2 & 5 & $76-10$ & $75-10$ & $79-10$ \\
\hline 40 & Quangng & 48863 & 15.1 & 108.8 & 8 & $76-10$ & $75-10$ & $79-10$ \\
\hline 41 & QuyNhon & 48870 & 13.8 & 109.2 & 4 & $76-10$ & $75-10$ & $79-10$ \\
\hline 42 & TuyHoa & 48873 & 13.1 & 109.3 & 11 & $76-10$ & $71-10$ & $79-10$ \\
\hline 43 & NhaTran & 48877 & 12.2 & 109.2 & 3 & $76-10$ & $71-10$ & $79-10$ \\
\hline 44 & PhanThi & 48887 & 10.9 & 108.1 & 9 & $76-10$ & $71-10$ & $79-10$ \\
\hline \multicolumn{9}{|c|}{ High Plain Central climate region (S2) } \\
\hline 45 & KonTum & 48865 & 14.4 & 108 & 538 & $76-10$ & $71-10$ & $79-10$ \\
\hline 46 & PlayKu & 48866 & 14 & 108 & 779 & $76-10$ & $71-10$ & $79-10$ \\
\hline 47 & BThuot & 48875 & 12.7 & 108.1 & 470 & $76-10$ & $71-10$ & $79-10$ \\
\hline 48 & DaLat & 48880 & 12 & 108.5 & 1509 & $76-10$ & $75-10$ & $79-10$ \\
\hline \multicolumn{9}{|c|}{ Mekong Delta climate region (S3) } \\
\hline 49 & TayNinh & 48898 & 11.3 & 106.1 & 9 & $78-10$ & $71-10$ & $79-10$ \\
\hline 50 & VungTau & 48903 & 10.4 & 107.1 & 4 & $78-10$ & $71-10$ & $79-10$ \\
\hline 51 & ConDao & 48918 & 8.7 & 106.6 & 6 & $78-10$ & $71-10$ & $79-10$ \\
\hline 52 & MocHoa & 48906 & 10.8 & 105.9 & 2 & $78-10$ & $71-10$ & $79-10$ \\
\hline 53 & MyTho & 48912 & 10.4 & 106.4 & 1 & $78-10$ & $71-10$ & $79-10$ \\
\hline 54 & ChauDoc & 48909 & 10.8 & 105.1 & 4 & $78-10$ & $71-10$ & $79-10$ \\
\hline 55 & CanTho & 48910 & 10 & 105.8 & 1 & $78-10$ & $71-10$ & $79-10$ \\
\hline 56 & SocTran & 48913 & 9.6 & 106 & 2 & $78-10$ & $71-10$ & $79-10$ \\
\hline 57 & RachGia & 48907 & 10 & 105.1 & 1 & $78-10$ & $71-10$ & $79-10$ \\
\hline 58 & CaMau & 48914 & 9.2 & 105.2 & 1 & $78-10$ & $71-10$ & $79-10$ \\
\hline 59 & PhuQuoc & 48917 & 10.2 & 104 & 3 & $78-10$ & $71-10$ & $79-10$ \\
\hline \multicolumn{9}{|c|}{ Island region (IS) } \\
\hline 60 & TruongS & 48920 & 8.7 & 111.9 & 3 & $78-10$ & $71-10$ & $79-10$ \\
\hline
\end{tabular}

Table 2.1 List of Vietnam stations used in the study. Data period (19xx-2010), where $\mathrm{xx}$ is the last two digits of the year, varying from 1971 to 2010. 


\subsubsection{Dynamic atmospheric reanalyses}

These four different reanalyses are used in a cross-checking process to recognise the presence of a semi-permanent vortex in the Southeast Asian Sea - Maritime Continent (SEAMC) which is introduced in detail in Chapter 6. Because every single reanalysis dataset was built by a different dynamic core, if the semi-permanent vortex is found in all these well-known reanalyses datasets then the conclusion on the vortex is more reliable.

\section{a. Japanese reanalyses}

Both the Japan Meteorological Agency (JMA) Climate Data Assimilation System (JCDAS) and the Japanese 55-year Reanalysis project (JRA-55) are used. JCDAS is an extension of the Japanese 25-year reanalysis (JRA-25, Onogi et al., 2007), in which normals are calculated from analyses for the period 1979-2004, and its products are available at http://jra.kishou.go.ja/JRA-25/index_en.html. JRA-55 is the second-generation Japanese reanalysis which spans 1958-2012. The major improvements of JRA-55 include a 4D-VAR data assimilation scheme, a new radiation scheme, higher model resolution $\left(1.25^{\circ}\right.$ $\mathrm{x} 1.25^{\circ}$ ), and reprocessing of satellite data (Ebita et al., 2011). Before January 2014, when JRA-55 had not been published, daily and monthly JRA-25 was used instead. JRA-55 is used for the study in Southeast Asian Sea - Maritime Continent (SEAMC) rainfall and vortex activity.

\section{b. European reanalyses}

ERA-Interim is the latest European Centre for Medium Range Weather Forecasts (ECMWF) global atmospheric reanalysis of the period 1979 to the present (Dee et al., 2011). The data can be retrieved through the ECMWF website at http://dataportal.ecmwf.int. Compared to previous versions, a new 4D-Var data assimilation, vertical levels increased from 23 to 37, higher horizontal grid resolution, improved model physics and quality control are added to the new version. To detect the SEASMC vortex (described in Section 2.4.4), $1.5^{\circ} \mathrm{x} 1.5^{\circ}$ latitude/longitude resolution monthly temperatures at $850 \mathrm{hPa}$, $700 \mathrm{hPa}, 500 \mathrm{hPa}$, and $300 \mathrm{hPa}$, and winds at the $10 \mathrm{-m}$ level and $850 \mathrm{hPa}$ for the period $1979-$ 2010 are taken in this study. 


\section{c. National Centers for Environmental Prediction (NCEP) reanalyses}

The NCEP-Department of Energy (NCEP-DOE) Reanalysis 2 project is the latest NCEP reanalysis project which aims to improve the NCEP reanalysis first generation (NCEP1). The model used has upgraded parameterization schemes of physical processes and higher horizontal resolution and covers the period 1979-2013. NCEP2 data is provided by the NOAA/OAR/ESRL PSD, Boulder, Colorado, USA, from their web site at http://www.esrl.noaa.gov/psd/.

\subsubsection{Rainfall merged satellite data}

Over land the Asian Precipitation - Highly Resolved Observational Data Integration Towards Evaluation of Water Resources (APHRODITE) is used. APHRODITE combined 5000-12000 stations from 1951 in the regions of Himalayas, Southeast Asia, and the mountainous region of Middle East through the real-time Global Telecommunication System, data sharing from individual National Centres for Hydrometeorological Services, and APHRODITE's own collection (Yatagai, 2012). In this study, we extract the monthly data from the $0.5^{\circ} \times 0.5^{\circ}$ daily data set in the latest version V1101.

Because of the lack of observation stations over the oceans, rainfall estimated from satellite information is a necessary but appropriate choice for climate studies in these regions. The Climate Prediction Center Merged Analysis of Precipitation (CMAP; Xie and Arkin 1997) data set is employed for studies of rainfall characteristics in REG3 in Chapter 4. Rain gauges, satellite data, and the NCEP-NCAR reanalyses data have been merged to produce the $2.5 \times 2.5^{\circ}$ gridded data globally. Only gridded points over the ocean in the REG3 domain are selected in the current study.

APHRODITE and CMAP data for the period 1979-2010 are used in this study.

\subsubsection{Typhoon tracks}

We use the International Best Tracks Archive for Climate Stewardship/World Meteorological Organisation (IBTrACS-WMO, Knapp et al. 2010) tropical cyclone data (http://www.ncdc.noaa.gov/oa/ibtracs/index.php?name=wmo-data) to detect typhoons in reanalyses data sets. Being the WMO official archiving and distribution resource, IBTrACS has advantages including being the most complete global set of historical 
typhoons, having undergone comparison between different agency products, and having several convenient multi-style formats. Sixty-two years (1952-2013) ${ }^{1}$ of typhoon activity over the SEAMC region is used in Chapter 6.

\subsection{Methods}

Fundamental statistical analyses and empirical statistics are conducted at different stages of the study to achieve the following objectives:

- Evaluate spatio-temporal relationships between predictands (in our study mostly are rainfall and 2-m temperature) and predictors (e.g.: SSTA, MSLP, U850) over the REG1, REG2, REG3, and Vietnam regions.

- Investigate climate variability, climate trends, and climate sub-region classification in Vietnam.

- Investigate tropical vortex activity over the REG2, REG3, and Vietnam regions.

- Define a global summer monsoon index.

\subsubsection{Averages}

Chapter 5 introduces a monsoon index which is found from standardized meteorological fields. Each field $\mathrm{x}$ is standardized by its mean and standard deviation.

$$
\begin{aligned}
& x_{\text {stdized }}=\frac{x-\bar{x}}{s_{x}} \\
& s_{x}=\sqrt{\frac{\sum_{i=1}^{n}\left(x_{i}-\bar{x}\right)^{2}}{n-1}}
\end{aligned}
$$

where $\bar{x}=\frac{1}{n}\left(x_{1}+x_{2}+\ldots .+x_{n}\right)$ is the average of the field, $n$ is number of data points.

To smooth out the daily data in the index computation, a five-day moving average is used. That means the day 1 in a time-series is treated by the average from day 1 to day 5 , the day 2 is the average value from day 2 and day 6 , and so on.

\footnotetext{
${ }^{1}$ Before the satellite era (1979), the reliability of typhoon best tracks is not as high as today
} 


\subsubsection{Pearson correlation}

Pearson correlation is one of the most conventional methods to detect teleconnections between climatic parameters. The method seeks a single-valued measure of association between two variables, say $x$ and $y$ during $n$ years. The Pearson correlation can be seen as the ratio of the sample covariance of $(\mathrm{x})$ and $(\mathrm{y})$ to the product of the two standard deviations:

$r_{x y}=\frac{\operatorname{COV}(x, y)}{s_{x} s_{y}}=\frac{n \sum x y-\sum x \sum y}{\sqrt{\left[n\left(\sum x^{2}\right)-\left(\sum x\right)^{2}\right]\left[n\left(\sum y^{2}\right)-\left(\sum y\right)^{2}\right]}}$

where $r_{x y}$ denotes sample correlation coefficient, $s_{x} s_{y}$ standard deviations are determined in (2.1).

The Pearson correlation is used in many parts of the thesis, e.g., Section 4.2 Chapter 4 investigates links between JRA-25 reanalyses and Vietnam temperature variations.

Although the Pearson correlation is often used, it is not used to seek the nonlinear relationship between the two variables and it is sensitive to outlying point pairs. Anscombe (1973) showed an example of the limitation of linear correlation. The four y variables in Figure 2.3 have the same mean (7.5), standard deviation (4.12), correlation (0.81) and regression equation $(y=3+0.5 x)$. Figure $2.3 a$ is what we expect to see; Figure $2.3 b$ represents a clear nonlinear relationship but it is not detectable by the Pearson correlation; and the last two data sets (Figure 2.3c,d) remind us the affection of outlier pairs.
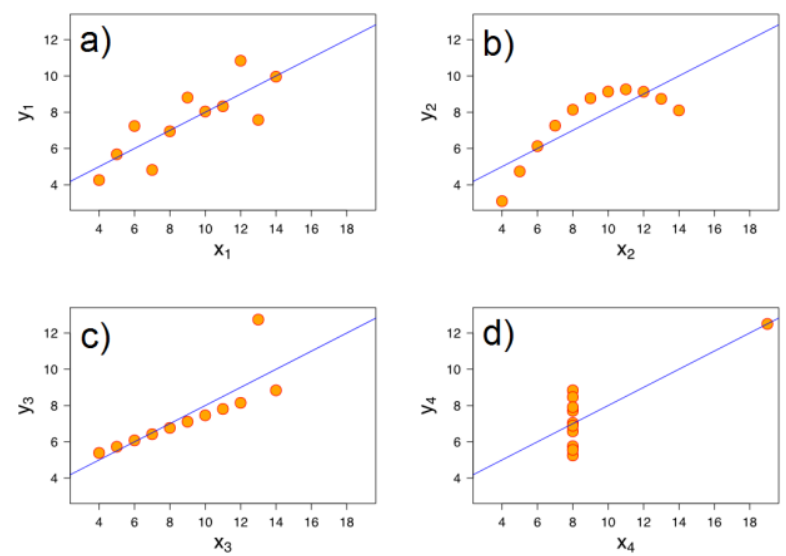

Figure 2.3: Anscombe's quartet. Sample diagrams in Pearson correlation analysis. 


\subsubsection{Significance of correlation coefficient}

The Pearson correlation coefficient tells us about the strength of the linear relationship between $\mathrm{x}$ and $\mathrm{y}$. However, the reliability of the linear model also depends on how many observed data points are in the sample. We need to look at both the value of the correlation coefficient $r$ and the sample size $n$, together.

After calculating the Pearson $r$ coefficient, we use a table of critical values (significance level $\alpha$ in two tails, usually $0.1,0.05$, or 0.01 ) to see whether the computed value of $\mathrm{r}$ is significant or not. If $r$ is not between the positive and negative critical values, then the correlation coefficient is significant. If $r$ is significant, we believe that there is a significant linear relationship between $\mathrm{x}$ and $\mathrm{y}$. We can use the regression line to model the linear relationship between $\mathrm{x}$ and $\mathrm{y}$ in the population (More detail in Section 4.2, 4.3).

\subsubsection{Climate trend detection}

There is no universally accepted best method for determining trends in climate variables (Moberg et al., 2006). Here we choose the nonparametric Mann-Kendall test to identify the existence of significant trends. Advantages of this test are that the dataset does not need to conform to any particular distribution, which is the most suitable for rainfall, and allows missing data to be assigned a value that is smaller than the smallest measured value in the dataset. The test compares the relative magnitudes of sample data rather than the data values themselves (Gilbert, 1987). If a significant trend is found, its slope and confidence interval are estimated using Sen's method (Sen, 1968). This statistical technique will be employed in several parts in this work. The description of the Mann-Kendal analysis and discussion on the uncertainties in trend detection in the in-situ stations are given in the appendix for methodologies.

To investigate the trend of rainfall in Vietnam, we propose the All-Vietnam summer monsoon Rainfall Index (AVRI) which collects all raingauge data in the Vietnam subregions and considers the whole country as one unit. The Central rainfall is established by

the same approach for all stations in Central. Specifically, the Central in this study is a region joining between N4 and S1 sub-regions in Table 2.1 and Figure 2.2, covering the domain $\left(10.9^{\circ}-20.4^{\circ} \mathrm{N}, 105.1^{\circ}-109.3^{\circ} \mathrm{E}\right)$. 


\subsubsection{Cluster analysis (K-means)}

K-means clustering is an algorithm that can classify objects (in this work, rainfall and temperature stations) into $\mathrm{K}$ groups by maximising between-cluster to within-cluster variance, using a Euclidean distance metric (Kidson, 1997, 2000; Huth, 2001). First, the given object is grouped into $\mathrm{K}$ (random) clusters. For each cluster the center-based point (centroid) is sought. Second, each point belonging to a given sub-data set is taken and associated it to the nearest centroid. The first random grouping is completed whenever no point is pending. Next, the $\mathrm{K}$ new centroids are re-calculated. Associated with each new centroid is its new set of satellite points; locations of $\mathrm{K}$ centroids at this step are different from the previous step. A loop has been generated until the K centroids do not change their locations. As the result, the distance between a data point and its cluster centre is to be minimized. The basic algorithm is composed by steps in Figure 2.4.

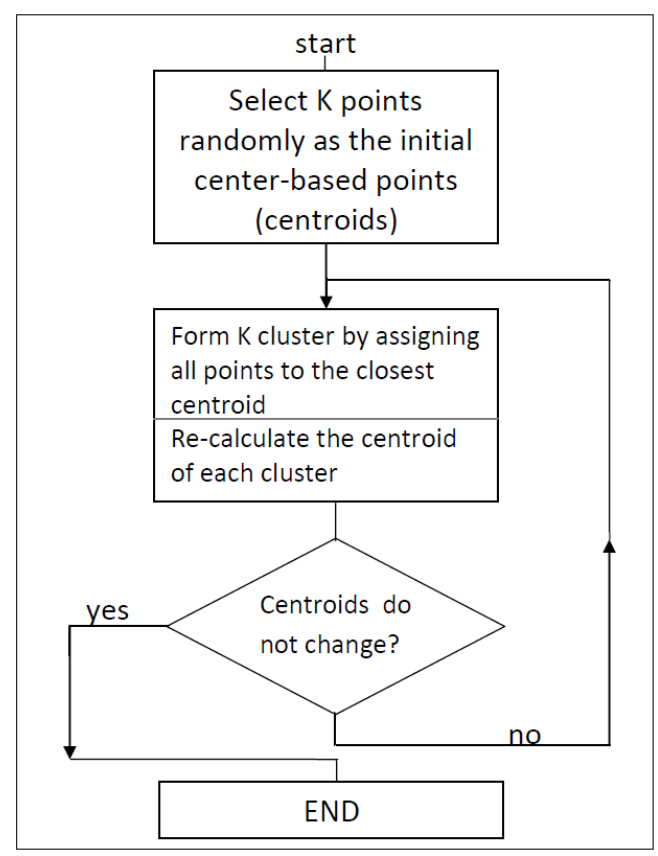

Figure 2.4. Basic K-means algorithm

However, K-means method is sensitive to the selection of the K-number. This cluster analysis technique is used to revisit a regional climate classification which was done by the Vietnamese experts' judgment. We have set K from 3 to 10, and select the best result. The "best" result will give us the most understandable climate sub-regions under the views of 
geophysical mechanisms. The K-mean cluster is applied in Chapter 4 to classify the subclimate regions in Vietnam.

\subsubsection{Tropical cyclone detection}

The Southeast Asian Sea is one of the regions in the world most affected by tropical cyclone (TC) activity. Damages are recorded annually. Obviously, TC activity is a notable regional climate feature. Here we investigate the TC occurrence frequency and its impact on regional rainfall (Chapter 6).

Criteria for detection of a tropical vortex are based on Nguyen and Walsh (2001). Four threshold criteria are used for detection of a vortex: wind speed at least $5 \mathrm{~ms}^{-1}$ at $10-\mathrm{m}$ level, vorticity at least $1 \times 10^{-5} \mathrm{~s}^{-1}$ at $850 \mathrm{hPa}$, total warm core temperature anomaly at $700 \mathrm{hPa}, 500 \mathrm{hPa}, 300 \mathrm{hPa}(\mathrm{T} 700+\mathrm{T} 500+\mathrm{T} 300)$ at least $0^{\circ} \mathrm{K}$, and temperature at $300 \mathrm{hPa}$ is greater than that of $850 \mathrm{hPa}$. In general the warm core and structure criteria were used to recognise the distinct difference between a tropical and high-latitude vortex. Here the domain of study is well inside the tropics and therefore these thresholds can be ignored. A vortex is considered to exist at a grid point if the relative vorticity and 10-m wind criteria are met there, or if they are met using vorticity and $10-\mathrm{m}$ wind averaged across the four closest neighbouring grid points.

\subsubsection{Multivariate methods}

This thesis makes extensive use of gridded meteorological fields (e.g. MSLP, or Vietnam rainfall at multiple sites) and of commonly-used techniques of multivariate analysis. One task in this study is to reduce the dimensionality of these fields to a few modes in such a way that the principal information is saved. Empirical orthogonal function (EOF) analysis extracts the highest-variance patterns in one field, and singular value decomposition (SVD) analysis seeks pairs of patterns in two fields that have maximal covariance. Detailed discussion of EOF and SVD techniques can be seen in Preisendorfer (1988), Von Storch and Navarra (1999), Jolliffe (2002), and Wilks (2011). Some

applications of the EOF and SVD analysis can be found in Renwick and Wallace (1995), Lotsch (2003), or Nguyen et al. (2014a). 


\section{a. Empirical Orthogonal Function (EOF) analysis}

Let the matrix of our variable of interest be $X(t, s)$, defined at t observations and s spatial positions. The value of $X$ at time $t_{i}$ and grid point $s_{j}$ is denoted $x_{i j}$ for $i=1, ., n$ and $j=1, \ldots$, p. $\mathrm{X}$ is then represented by the data matrix:

$$
X(t, s)=\left(x_{1}, x_{2}, \ldots . ., x_{n}\right)^{T}=\left(\begin{array}{llll}
x_{11} & x_{12} & \ldots & x_{1 p} \\
x_{21} & x_{22} & \ldots & x_{2 p} \\
\ldots & \ldots & \ldots & \ldots \\
x_{n 1} & x_{n 2} & \ldots & x_{n p}
\end{array}\right)
$$

where $x_{t}=\left(x_{t 1}, x_{t 2}, \ldots, x_{t p}\right)^{T}, t=1, . ., n$, represents the map, or the value of $X$ at time $t$. Let denote by $\bar{x}_{.1}$ the time average of $\mathrm{X}$ at the $\mathrm{i}$ 'th spatial gridpoint. The time average and the climatology of $\mathrm{X}$ are given by:

$$
\begin{aligned}
& \bar{x}_{. i}=\left(\bar{x}_{.1}, \ldots . ., \bar{x}_{. p}\right)=\frac{1}{n} \sum_{k=1}^{n} x_{k i \bullet} \\
& \bar{x}=\left(\bar{x}_{.1}, \ldots, \bar{x}_{. p}\right)=\frac{1}{n} l_{n}^{T} X
\end{aligned}
$$

The anomaly field is defined at $\left(\mathrm{t}, \mathrm{s}_{\mathrm{k}}\right), \mathrm{t}=1, \ldots, \mathrm{n}$, and $\mathrm{k}=1, \ldots, \mathrm{p}$, by

$$
x_{t k}^{\prime}=x_{t k}-\bar{x}_{. k}
$$

(2.6) can be written in matrix form:

$$
Y=X^{\prime}=X-1_{n} \bar{x}=\left(I_{n}-\frac{1}{n} 1_{n} 1_{n}^{T}\right) X=H X
$$

where $\bar{x}_{. i}$ is the time average at the i'th spatial grid point,

$1_{\mathrm{n}}=(1, \ldots, 1)^{\mathrm{T}}$ is the vector of length $\mathrm{n}$ containing only ones.

$\mathrm{I}_{\mathrm{n}}$ is the $(\mathrm{n} \times \mathrm{n})$ identity matrix.

EOFs of $\mathrm{Y}$ are obtained by finding the eigenvalues of the covariance matrix $\mathbf{C}$ :

$$
C=\frac{1}{n} Y^{T} Y=U \Lambda U^{T}
$$


where $\mathrm{U}$ and $\Lambda$ are the orthonormal matrix of eigenvectors and the diagonal matrix of the eigenvalues, respectively. The EOFs are therefore the columns of U. The first EOF (EOF1) is the unit-length vector defining the linear combination of the columns of $\mathrm{Y}$ which has maximal temporal variance. Subsequent EOFs are determined similarly, but with the added constraint that they must be orthogonal to all other EOFs. The matrix $\Lambda$ of eigenvalues contains the variance associated with each EOF, in descending order.

The projection of the anomaly field $\mathrm{Y}$ onto $\mathrm{U}$ determines the principal components (PCs) $\mathrm{Z}$ :

$$
\mathrm{Z}=\mathrm{YU}
$$

$\mathrm{Z}$ is also known as the EOF expansion coefficients, EOF time series amplitude, PCs score, or PC time series. Figure 2.5 is a quick illustration on the EOF technique. One common goal of EOF analysis is to reduce the dimensionality of the data. In practice only first $p$ EOF modes are kept, where $\mathrm{p}$ is much smaller than the rank $\mathrm{n}$ of $\mathrm{Y}$. There is no universal rule for truncation or the choice of $p$, but in many studies $p$ is chosen so that the first $p$ EOFs account for about $80 \%$ of variance of $Y$ (Hannanchi et al., 2007).

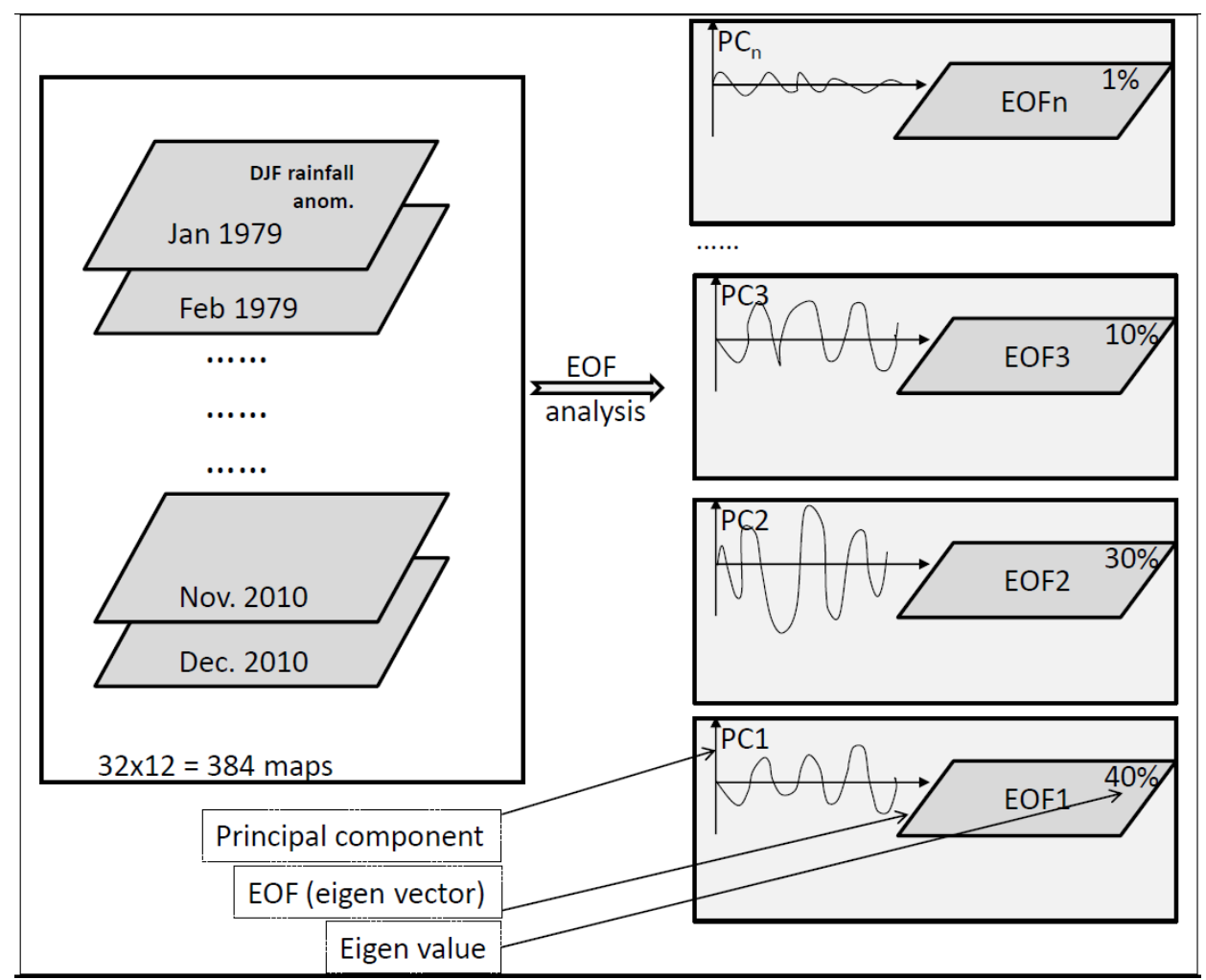

Figure 2.5. A demonstration of EOF analysis. 


\section{b. Singular Value Decomposition Analysis (SVD)}

Assume we have a set of gridded variables; say MSLP, U850, U200, temperature and geopotential height at $850 \mathrm{hPa}$, surface temperature, convective rainfall, and OLR. We wish to find the variables which have strongest relationships with gridded rainfall fields. SVD analysis can be used in this circumstance. Each of these variables is employed in turn in SVD analyses to seek coupled patterns with rainfall. The best variables (those with the strongest coupling) will be considered and selected based on the statistics of covariances between those coupled SVD patterns. This is the first step in the defining monsoon index procedure which will be introduced thoroughly in Section 2.3.6 and Chapter 4.

Bretherton et al. (1992) and Wallace et al. (1992) described a matrix operation to obtain singular values derived from paired sets of fields. Let denote the two variables are X and Y respectively, where $\mathrm{X}$ and $\mathrm{Y}$ having $\mathrm{n}$ rows by q columns. SVD is a decomposition of the sample $\mathrm{X}-\mathrm{Y}$ covariance matrix $\mathrm{C}_{\mathrm{XY}}$ :

$$
C_{X Y}=\frac{1}{n} X^{T} Y=U S V^{T}=\sum_{i=1}^{q} \sigma_{i} u_{i} v_{i}^{T}
$$

where the columns of the orthonormal matrix $U(V)$ are the left (right) singular vectors $u_{i}$ $\left(v_{i}\right)$, and $\mathrm{S}$ is a real diagonal matrix of singular values $\sigma_{i}$ such that $\sigma_{i} \geq \sigma_{q}$ with $\{i=1,2, . . q\}$. The pair of pattern whose amplitude time series account for the maximal covariance between $\mathrm{X}$ and $\mathrm{Y}$ is determined by the first combination $\sigma_{1} \mathrm{u}_{1} \mathrm{v}_{1}{ }^{T}$. The difference between singular vectors in SVD and EOF analysis is that the spatial form of singular vectors in SVD depends on the structure of the covariance between two fields $\mathrm{X}$ and $\mathrm{Y}$ whilst the one EOF depends on the structure of the variance of only one field. Time series $a_{i}$ and $b_{i}$ of the PCs are calculated by projecting $\mathrm{X}$ and $\mathrm{Y}$ onto the $\mathrm{u}_{\mathrm{i}}$ and $\mathrm{v}_{\mathrm{i}}$, correspondingly. 


\section{CHAPTER 3}

\section{GENERAL CLIMATE FEATURES}

Look deep into nature, and then you will understand everything better.

Albert Einstein

This chapter is an introduction of the three main climate elements, temperature, rainfall and wind, in Vietnam. On a bigger spatial scale, synoptic patterns which control the weather and climate in the country are also described. The chapter ends with an overview of extreme weather and climate events in Vietnam. A part of this chapter was presented in Nguyen et al. (2014a). 


\subsection{Vietnam geography}

Located in the centre of two main tropical monsoon areas, the South Asian and the East Asian monsoons, and lying from $8.30^{\circ} \mathrm{N}$ to $23.22^{\circ} \mathrm{N}$, Vietnam has a typical tropical monsoon climate with a seasonal reversal in atmospheric circulation and precipitation associated with the thermal contrast in east-west or land-sea heating. The territory includes an extensive land area (about 332,000 $\mathrm{km}^{2}$ ) and a greater maritime area (about 4.5 million $\mathrm{km}^{2}$ ) in which are located many islands.

Mountains above $1000 \mathrm{~m}$ are found in the Northwest, Central, and Central Highland regions (Figure 1.1). Truong-Son mountain range, on average 600-1000m in height and about $1000 \mathrm{~km}$ wide separating Vietnam and Laos, is a special topography feature in Central Vietnam. From May to September, when the southwesterly prevails, most of the central stations are in the rain shadow of the Truong-Son mountain ridge. During this time the Foehn effect, resulting in dry and hot westerlies in the lee of the Truong-Son ridge (downwind side), raises the average temperature on the central coast. People in Central Vietnam usually name this hot and dry weather as Laos winds - winds coming from Laos. Months later, when the prevailing winds are north-easterly, those stations are on the windward side of the Truong-Son mountains and as a result, increased rainfall amounts are recorded in this region.

Vietnam's extensive water resources consist of a dense network of rivers. From north to south there are nine major river systems, including the Mekong River in the south, each with a basin area of at least $10,000 \mathrm{~km}^{2}$. Besides the Southeast Asian Sea, two large gulfs, the Tonkin gulf (located in the north) and the Thailand gulf (located in the south) are a significant part of Vietnam's hydrological resource.

In general, summer months and the rainy season start together in May but they generally end at different times. The rainy season ends in November, while summer season ceases about two months earlier (Figure 1.3). The mean temperature varies from $18^{\circ}-28^{\circ} \mathrm{C}$ and the mean accumulated precipitation during the rainy season is greater than $150 \mathrm{~mm}$ per month (Figure 3.1). 


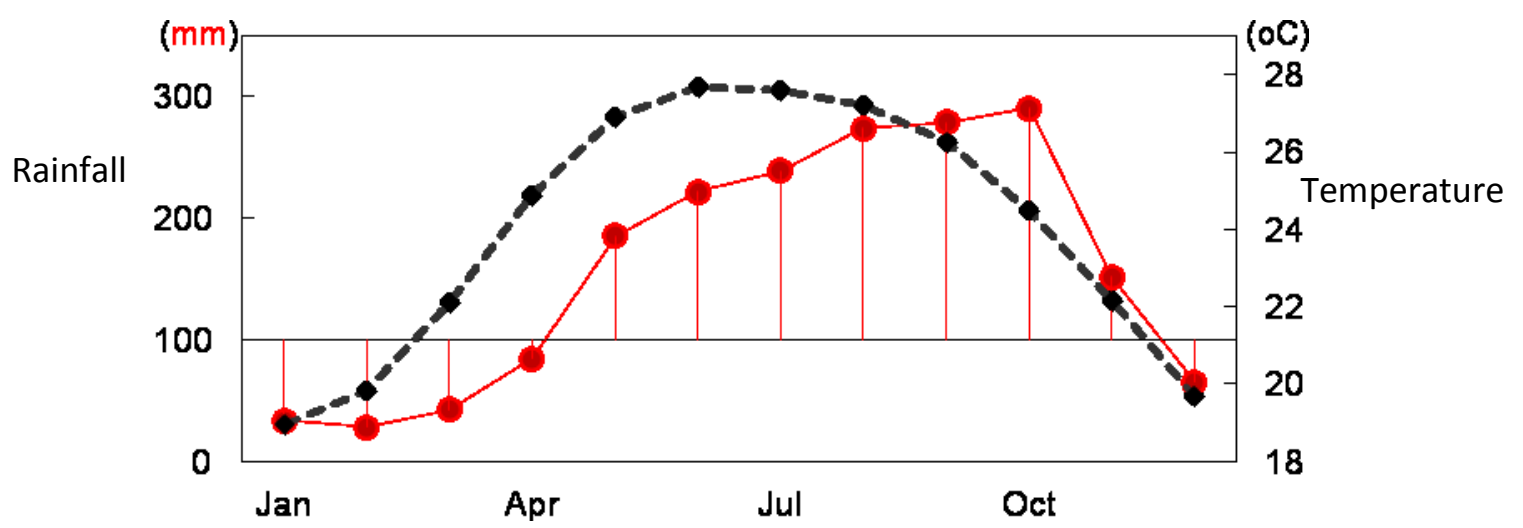

Figure 3.1. Monthly long-term average temperature (dashed black line) and rainfall (solid red line) in Vietnam.

\subsection{General circulation}

In summer, the climate of the Southeast Asia region (SEA) is dominated by the South Asian monsoon. This is also known as the south-west monsoon which is hot and wet, especially in the southern provinces of Vietnam. In the lower troposphere, at $850 \mathrm{hPa}$, the heat low over the northern Bay of Bengal and Pakistan (Asiatic Low), the subtropical high over the western North Pacific, and the cold anticyclone over the Australian continent (Australian High) are the main seasonal pressure centres that influence the monsoon (Figure 3.3, left panels, and Appendix Figure AF1). In the upper troposphere (200hPa), a high lies over the northern Bay of Bengal and westerly jets over northern Australia. The southwest monsoon develops in response to the eastward extension of the Asiatic Low, the northward movement of the subtropical high (especially its associated ridge) into the Western Pacific, and the strengthening of the Australian High which is associated with low-level cross-equatorial flow between $40^{\circ}-110^{\circ} \mathrm{E}$. The northward movement begins in early May over the Vietnam - Indochina region and ends in late July-August over northern China (Wang and LinHo, 2002). Joining with the northward motion of the subtropical high, from the southwestern flank, a southeasterly flow develops in the northern provinces of Vietnam. Beyond the southwest monsoon, the southeasterly is a special characteristic of the summer monsoon in Vietnam. In many cases, it is the southwestern flank of the subtropical high that steers tropical cyclones ashore in the Southeast Asia region. Tropical cyclones are a very typical hazard in the summer monsoon season in this region. 

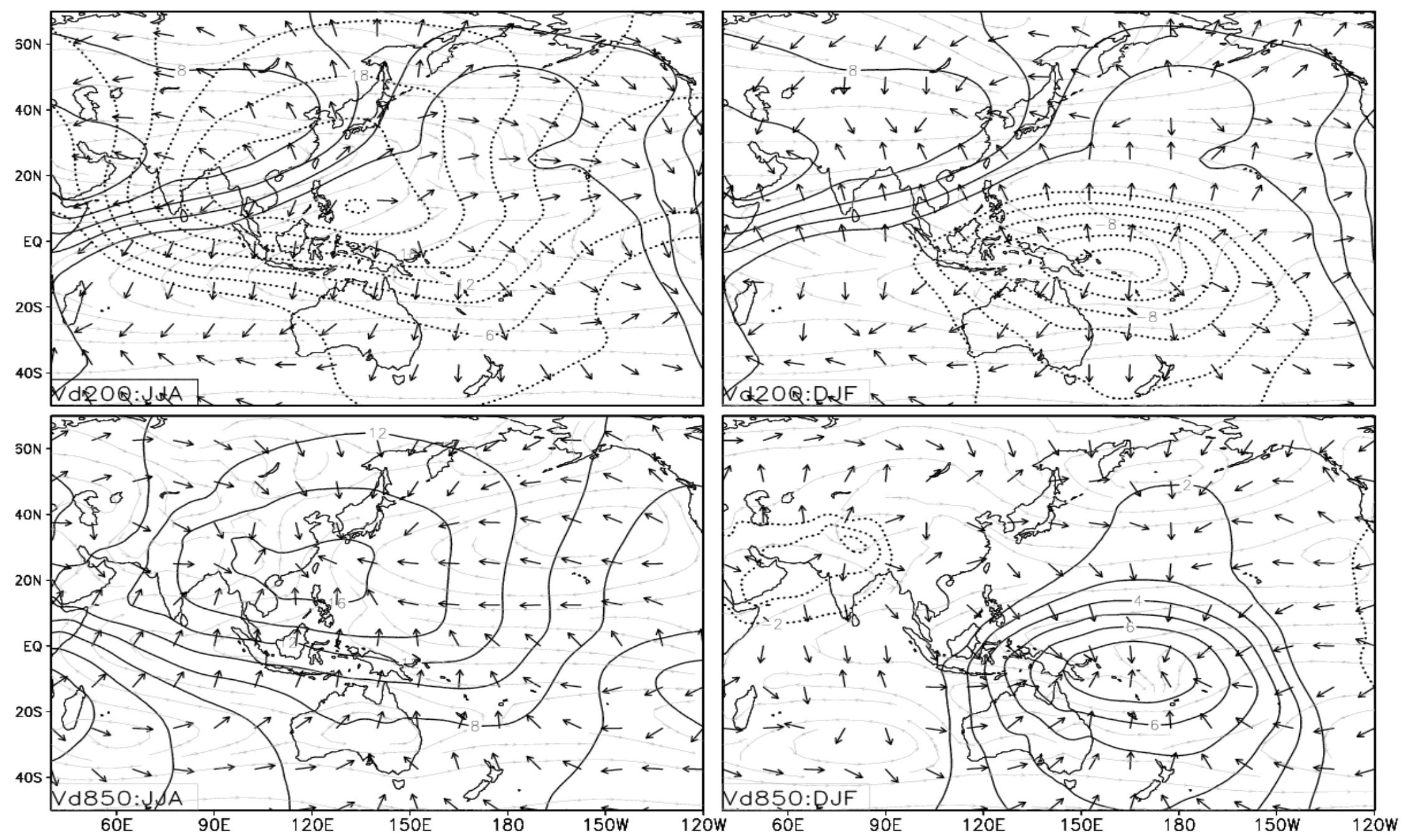

Figure 3.2. Velocity potential ( $1 \mathrm{e} 6 \mathrm{~m}^{2} / \mathrm{s}$, contours) and divergent component of vector winds at $850 \mathrm{hPa}$ (bottom) and 200hPa (top). JCDAS 19792010 average for JJA (left) and DJF (right). Gray contours: Streamlines. 
In winter the climate is affected by the East Asian monsoon, which is cold and dry especially in the north and the central areas of Vietnam (Figure 3.3, right panels, and Appendix Figure AF1). Winter usually extends from November to March. By midSeptember, the changes from cyclonic to anti-cyclonic of the low-level circulation pattern over the Asiatic continent have started, allowing the polar front to move south. As the boreal winter approaches, a strong surface high pressure system develops over East Asia, and is centred southwest of Lake Baikal, over the region bounded by $45^{\circ}-55^{\circ} \mathrm{N}, 90^{\circ}-105^{\circ} \mathrm{E}$. This Asiatic High (Siberian High) is sustained by strong radiational cooling and is consistent with cold convection from the Arctic. As the Asiatic High strengthens and the Australian Low deepens, the pressure gradient increases and the northeasterly flows intensify, following the shape of the East-Asian coast. The winter monsoon blows out of the Siberian High, sweeping the East Asian continental region, and becomes cold surges at lower latitudes; this process begins in the far north and moves progressively south over time (Chang et al., 2006). Passing on to Vietnam, cold surges accompany squall lines, gales, showers, and a sharp drop in temperature. A major surge may take six to seven days before ending at near-equatorial locations. Annually, there are 23-27 such fronts crossing Vietnam's territory; each cold surge might drop the average daily surface temperature by at least $3-5^{\circ} \mathrm{C}$ on two consecutive days and remain for three to five days on average (NCHMF, 2009). A strong cold surge hitting Vietnam and the SEAS region during the peak episode of the northeast monsoon season is shown in Fig. 3.4. Other weather surface analysis maps which show the synoptic situation on consecutive days are presented in the Appendix Figure AF2. During the first 24 hours, $15-16^{\text {th }}$ December 2009, the maximum and average surface temperature in Lang station (station code 48820 ), dropped by $8.1^{\circ} \mathrm{C}$ and $5.4^{\circ} \mathrm{C}$, respectively. On $17^{\text {th }}$ December, these temperatures decreased by $2.2^{\circ} \mathrm{C}$ and $2.5^{\circ} \mathrm{C}$ compared to previous day. Overall, it is these cold surges that significantly reduce the winter temperature and, as a result, increase the annual temperature amplitude. 


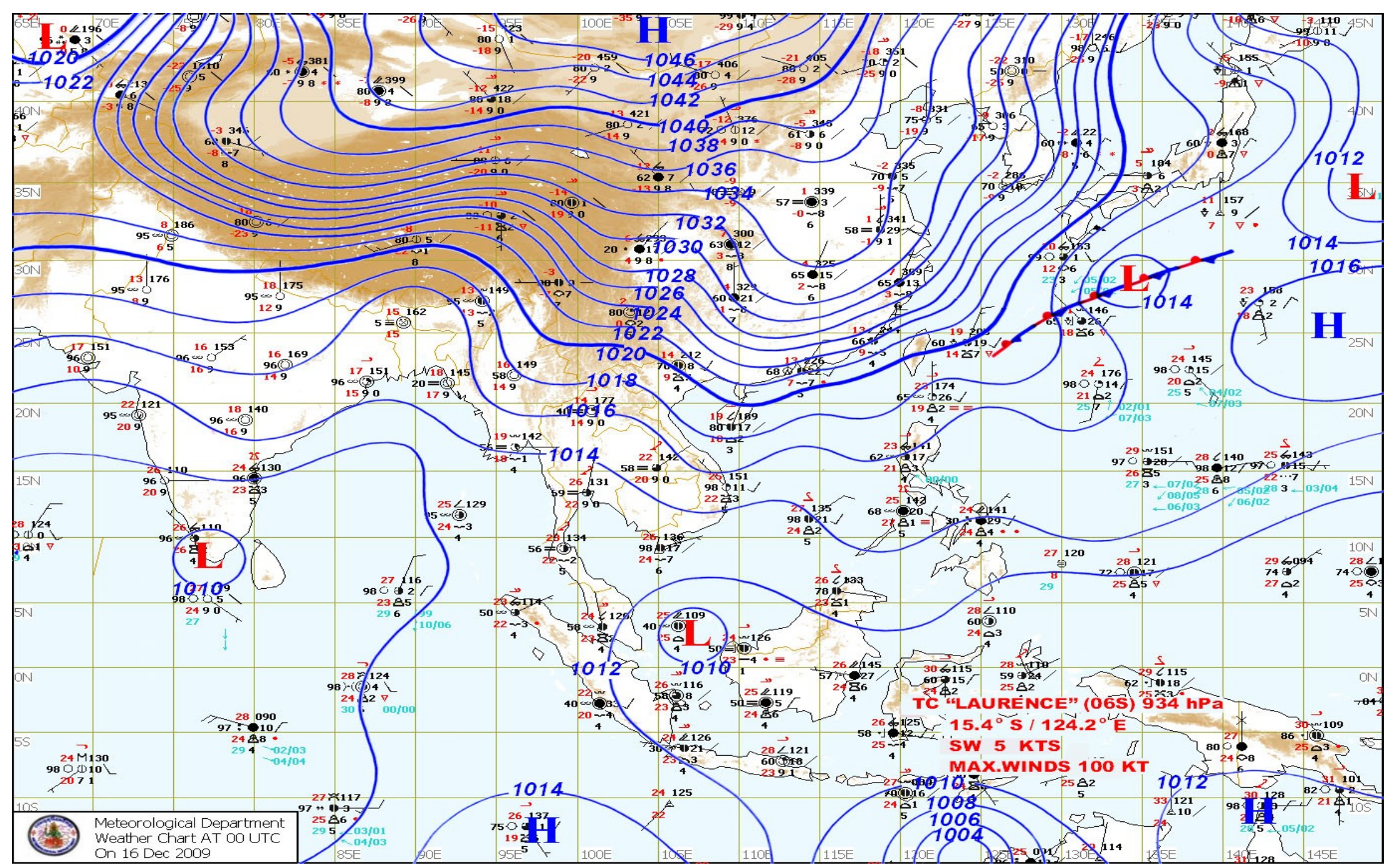

Figure 3.3. Surface analysis map of a typical cold surge hitting Vietnam and the SEAS region on 00UTC 16 December 2009. Blue contours: surface pressure $(\mathrm{mb})$. Capital L and $\mathrm{H}$ stand for low and high pressure centre. This map is provided by the Thai Meteorological Department. 
In addition, there are significant interactions between the two hemispheres in the form of cross-equatorial flows which contribute to the weather - climate regime in SEA. This crossequatorial flow transports moisture and heat from the Australian region to SEA in summer and the reverse transportation occurs in boreal winter. The local Hadley circulation is responsible for much of the transport in summer and winter. Such persistent large-scale overturning is a key characteristic of monsoons (Trenberth et al., 2000b).

\subsection{Temperature}

Annual temperatures in Vietnam show wide variations, large amplitudes occurring in the north (mostly resulting from very low minimum temperatures) and smaller amplitudes in the south. Across Vietnam, the January average temperature varies considerably from $2{ }^{\circ} \mathrm{C}-26^{\circ} \mathrm{C}$, and gradually increases from North to South. Meanwhile that of July varies from $20^{\circ}-30^{\circ} \mathrm{C}$ with no obvious latitudinal pattern in temperature variations. Along the Central coastal region, from about $10^{\circ} \mathrm{N}$ to $21^{\circ} \mathrm{N}$, the annual temperature decreases about $0.4^{\circ} \mathrm{C}$ per degree of latitude. The maximum annual temperature is about $27^{\circ} \mathrm{C}$ at Truong-Sa station (WMO code \# 48920, Chapter 2), and the minimal annual temperature, about $8^{\circ} \mathrm{C}$, is recorded at the Hoang-LienSon mountain, the highest mountain (about $3100 \mathrm{~m}$ ) in the north (Figure 3.1).

The diurnal variation of temperature shows the same pattern irrespective of the geographical location. The daily temperature deviation is nearly the same in all the geographical regions: it reaches its lowest in early morning or at dawn, increases gradually before reaching its peak by late midday, then decreases continuously throughout the night. In general, the diurnal amplitude of temperature is always above $6^{\circ} \mathrm{C}$, except in mountainous regions and islands.

Being a tropical country, however, the winter monsoon brings a reduction in temperature not experienced by any neighbouring regions; Vietnam has a "cold" winter (Ramage, 1968). In many places, especially in the northern mountainous provinces, recorded temperatures fall below $0^{\circ} \mathrm{C}$. The absolute minimum temperature that has been recorded is $-3.7^{\circ} \mathrm{C}$ in the HoangLien-Son mountain on $14^{\text {th }}$ December, 1975. In summer, hot spells are a special climate feature in North and Central Vietnam. The absolute maximum temperature during those days is usually above $40^{\circ} \mathrm{C}$. Some records of extreme temperatures are seen in Table 3.1. 


\begin{tabular}{|c|c|c|c|c|c|}
\hline \multirow[t]{2}{*}{ Regions } & \multirow{2}{*}{$\begin{array}{l}\text { Station } \\
\text { (WMO code) }\end{array}$} & \multicolumn{2}{|c|}{ Minimum temp $\left({ }^{\circ} \mathrm{C}\right)$} & \multicolumn{2}{|c|}{ Maximum temp. $\left({ }^{\circ} \mathrm{C}\right)$} \\
\hline & & Value & Date & Value & Date \\
\hline \multirow[t]{2}{*}{$\mathrm{N} 1$} & 48800 & 3.4 & 2Jan1974 & 42.5 & May1928 \\
\hline & 48806 & -0.5 & 18Jan1961 & 38.0 & 13Мay1966 \\
\hline \multirow[t]{2}{*}{$\mathrm{N} 2$} & 48802 & -3.2 & 14Dec1975 & 29.6 & 8Aug1981 \\
\hline & 48830 & -2.1 & 15Jan 1963 & 37.9 & 12May1966 \\
\hline \multirow[t]{2}{*}{ N3 } & 48820 & 2.7 & 12Jan1955 & 40,4 & 13Jun 1949 \\
\hline & 48826 & 4.5 & 14Feb1968 & 38.7 & 3May1994 \\
\hline \multirow[t]{2}{*}{ N4 } & 48845 & 5.2 & 24Dec1999 & 40.9 & 11Jul1977 \\
\hline & 48852 & 10.2 & 30Jan1992 & 41.4 & 15May1983 \\
\hline \multirow[t]{3}{*}{ S1 } & 48855 & 15.2 & 25Dec1999 & 40.5 & 4May1983 \\
\hline & 48877 & 15.1 & Dec1975 & 37.9 & 7Aug1976 \\
\hline & 48887 & 15.7 & 20Jan1976 & 37.7 & 3May1994 \\
\hline $\mathrm{S} 2$ & 48875 & 7.4 & 1955 & 39.4 & April 1983 \\
\hline \multirow[t]{2}{*}{ S3 } & 48903 & 18.1 & 29Jan1993 & 36.2 & 21April1990 \\
\hline & 48914 & 16.8 & 30Dec1975 & 38.2 & 16May1983 \\
\hline
\end{tabular}

Table 3.1. Extreme daily temperatures at some stations (listed in Chapter 2) in Vietnam.

\subsection{Rainy season}

The earliest onset of the Asian summer monsoon is usually observed in the central Indochina Peninsula region (Vietnam, Laos, and Cambodia) in late April and early May (Wang, 2006). The rainy season usually commences in May and ends in October-November. In many provinces in Vietnam, the rainy season starts in April-May, but sometimes it starts as early as March. The monsoon then shifts northward through a series of dry and wet phases. Each phase lasts from two to ten days, from May through August, progressing from Vietnam Taiwan in May; to the Yangzi River, China and Japan in June; and to North China and Korea in July-August. This seasonal rain is known as Mei-yu in China, Changma in Korea, or Bai-u in Japan. When the monsoon ends in July-August, the rain belt begins to move back over Indochina and the Southeast Asia region (Wang, 2006). The months of peak rainfall for each sub-region is showed in Figure 3.4. 

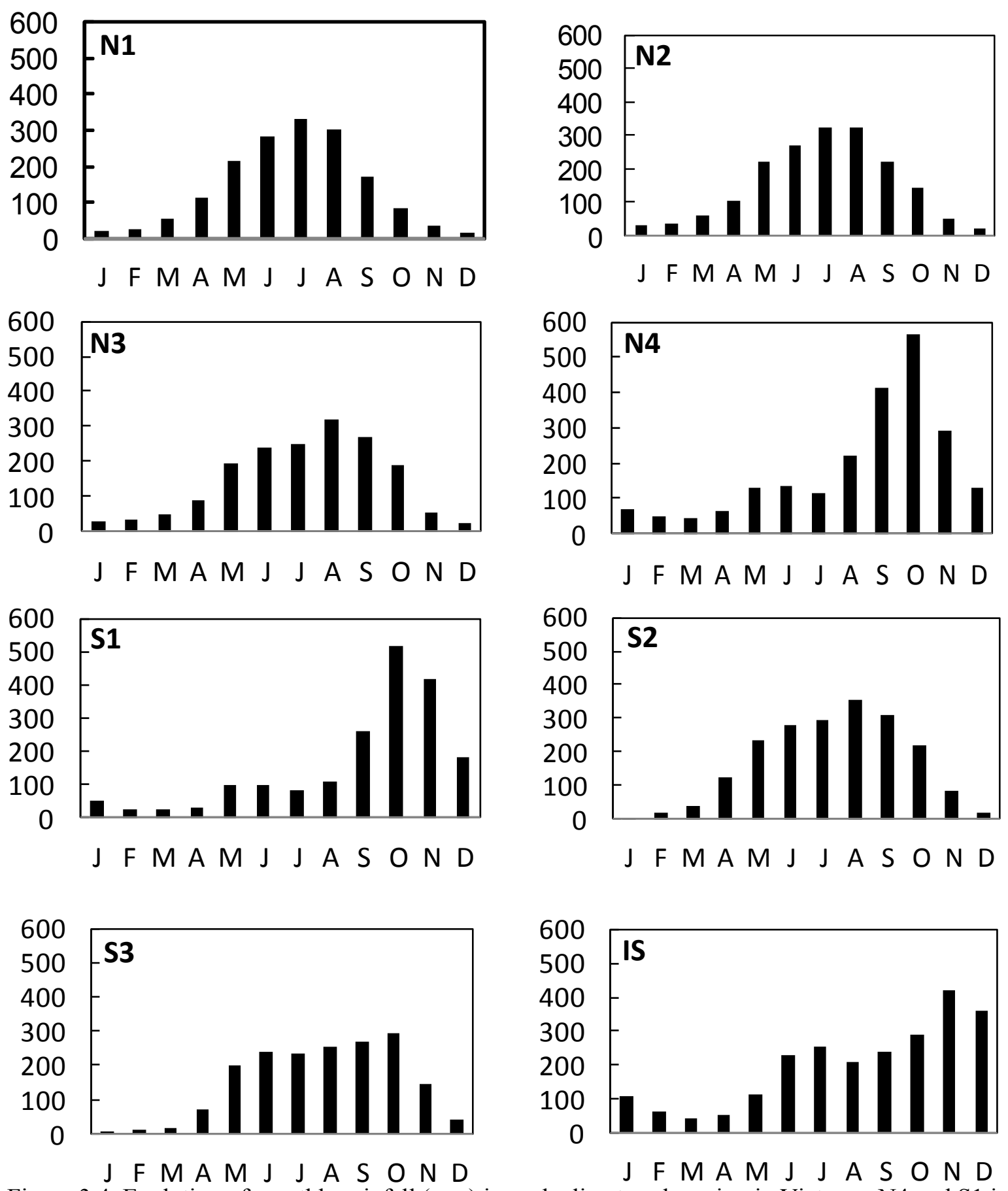

Figure 3.4. Evolution of monthly rainfall $(\mathrm{mm})$ in each climate sub-region in Vietnam. N4 and $\mathrm{S} 1$ is the coastal region that has the late rainy season till November. IS is the Truong-Sa station $\left(8.7^{\circ} \mathrm{N}\right.$, $\left.111.9^{\circ} \mathrm{E}\right)$. 
Those stations north of $17-18^{\circ} \mathrm{N}$ have high rainfall in the June-September period. Peak rainy months appear to move from north to south with time, and coincide with the southward migration of the subtropical ridge and ITCZ from July-September. The total rainfall amount differs markedly between dry and wet seasons, with a large annual range of precipitation that defines the amplitude of annual variation (Figure 3.4). The ratio of total rainfall in the dry to the rainy season, during the last 40 years, is about one to five; the contribution of precipitation in the rainy season to the annual rainfall in Vietnam is greater than $80 \%$.

\subsection{Winds}

\subsubsection{Wind direction}

One of the key monsoon features is the seasonal change of wind directions. In Vietnam, the prevailing wind direction in winter is from the northern quadrant (northwest, north, and northeast), and in summer is from the southern quadrant (southwest, south, and southeast).

The frequency of the prevailing wind in January and July at island stations can reach to $60-70 \%$, or even higher, whilst that of continental stations does not generally exceed $50 \%$, especially in the farthest mountains and midland areas. For example, in Truong-Sa (48920) and Bach-Long-Vy (48839) stations (the East coast island stations) the north-easterly in January takes $61 \%$ and $70 \%$ of wind frequency, respectively. These directions and frequencies change, by distance, when winds approach the continent. The prevailing winds are changed to northerly/north-easterly in the northern coastal delta (N3-subregion), northwesterly in the Central, and south-easterly in the South. Figure 3.5 below demonstrates the variation of wind directions in winter(January) and summer(July) at three stations, one in the north (Hanoi, \#48820), one in Central (Danang, \#48855), and one in the south (Ho-Chi-Minh city, $\left.\left(10.82^{\circ} \mathrm{N}-106.67^{\circ} \mathrm{E}\right)\right)$. 

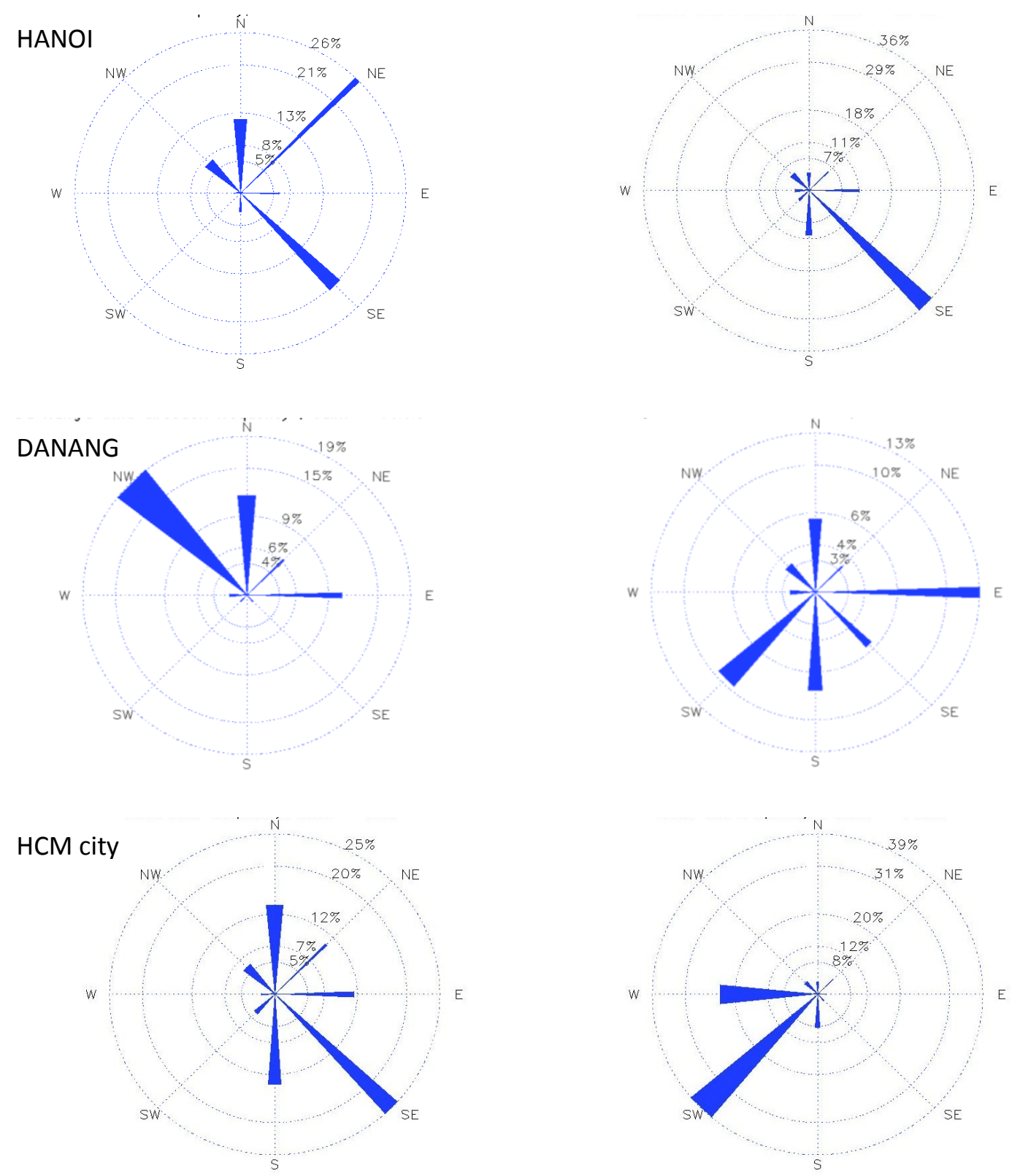

Figure 3.5. Thirty two year average (1979-2010) distribution of wind directions in Ha-Noi (48820), Da-Nang (48855), and Ho-Chi-Minh city (\#48900, $\left.10.82^{\circ} \mathrm{N}-106.67^{\circ} \mathrm{E}\right)$ represent the North, Central, and South stations. From left to right is the prevailing wind direction in January and July. The calm wind frequencies for those two months are $20.1 \%$ and $17.4 \%$ (Ha-Noi), $44.1 \%$ and $46.1 \%$ (Da-Nang), and 8\% and 10.5\% (Ho-Chi-Minh city), respectively. 


\subsubsection{Wind speed}

The annual average wind velocity is above $4 \mathrm{~m} \cdot \mathrm{s}^{-1}$ in islands, $1-2 \mathrm{~m} \cdot \mathrm{s}^{-1}$ in the north, 1.5$2.5 \mathrm{~m} . \mathrm{s}^{-1}$ in the northern Central, and 1.5-4.0 m.s ${ }^{-1}$ in the southern Central and the south. Wind speed in the northeast and northwest mountainous regions is not as strong as that in the Northern Deltas (N3-N4 sub-region); however, in the Highlands (S2 sub-region) it is higher than in the southern Central and the south. Regarding the average wind velocity, it is difficult to identify the common features of differences among seasons. In many areas, the winter winds are stronger than that of the summer winds, while in some places, the summer winds are stronger than the winter winds.

\subsection{Extreme weather events}

\subsubsection{Tropical cyclone}

The territory of Vietnam often suffers from the direct impacts of tropical cyclones, typhoons from the Western Pacific and/or from the SEAS region. Figure 1.4 shows the track of all known typhoons around the world from 1851 to 2006. We can see that Vietnam has been a favoured destination for tropical cyclones over time. Crossing the Philippines, then SEAS, these tropical cyclones hit the coastal areas of Vietnam, causing heavy rainfall, flood, and inundation. Rainfall induced by storm can reach $200-300 \mathrm{~mm}$ over 24hours in many places, particularly the central coast, and the maximum wind velocity may reach $55 \mathrm{~m} . \mathrm{s}^{-1}$

In the last 32 years (1979-2010) 334 typhoons/tropical cyclones (TC)/tropical lows or depressions (TD) that have struck Vietnam (Appendix Figure AF3). This severe weather phenomenon often happens from May to December, with July, August, September, October, and November being the months with the highest frequencies of storms (Table 3.2).

\begin{tabular}{llllllllllllll}
\hline Month & Jan & Feb & Mar & Apr & May & Jun & Jul & Aug & Sep & Oct & Nov & Dec & Year \\
\hline Freq. & $\mathbf{0}$ & $\mathbf{0}$ & $\mathbf{0 . 1}$ & $\mathbf{0 . 2}$ & $\mathbf{0 . 6}$ & $\mathbf{1 . 0}$ & $\mathbf{1 . 7}$ & $\mathbf{1 . 6}$ & $\mathbf{1 . 9}$ & $\mathbf{1 . 5}$ & $\mathbf{1 . 3}$ & $\mathbf{0 . 5}$ & $\mathbf{1 0 . 4}$ \\
\hline
\end{tabular}

Table 3.2. Monthly frequency of tropical cyclones directly impacts to Vietnam (1979 -2010). 
Along the coastline, the storm season associates consistently with the ITCZ movements; it makes landfall in North from June to September, Central region from July to December, and South from November to December.

Figure 3.6 illustrates a tropical storm; with the international name of PARMA-0917/2009. This TC was upgraded from a tropical depression over the North Western Pacific. Entering SEAS on the morning of 4 October, PARMA moved north-northwest then west-northwest. At O0UTC 5 November, PARMA was at $20,1^{\circ} \mathrm{N}-119,3^{\circ} \mathrm{E}$ with the maximum wind intensity of $90-100 \mathrm{~km} / \mathrm{h}$. Since it interacted with the typhoon MELOR, PARMA was almost stationary before moving east-southeast and southeast, then going out of SEAS.

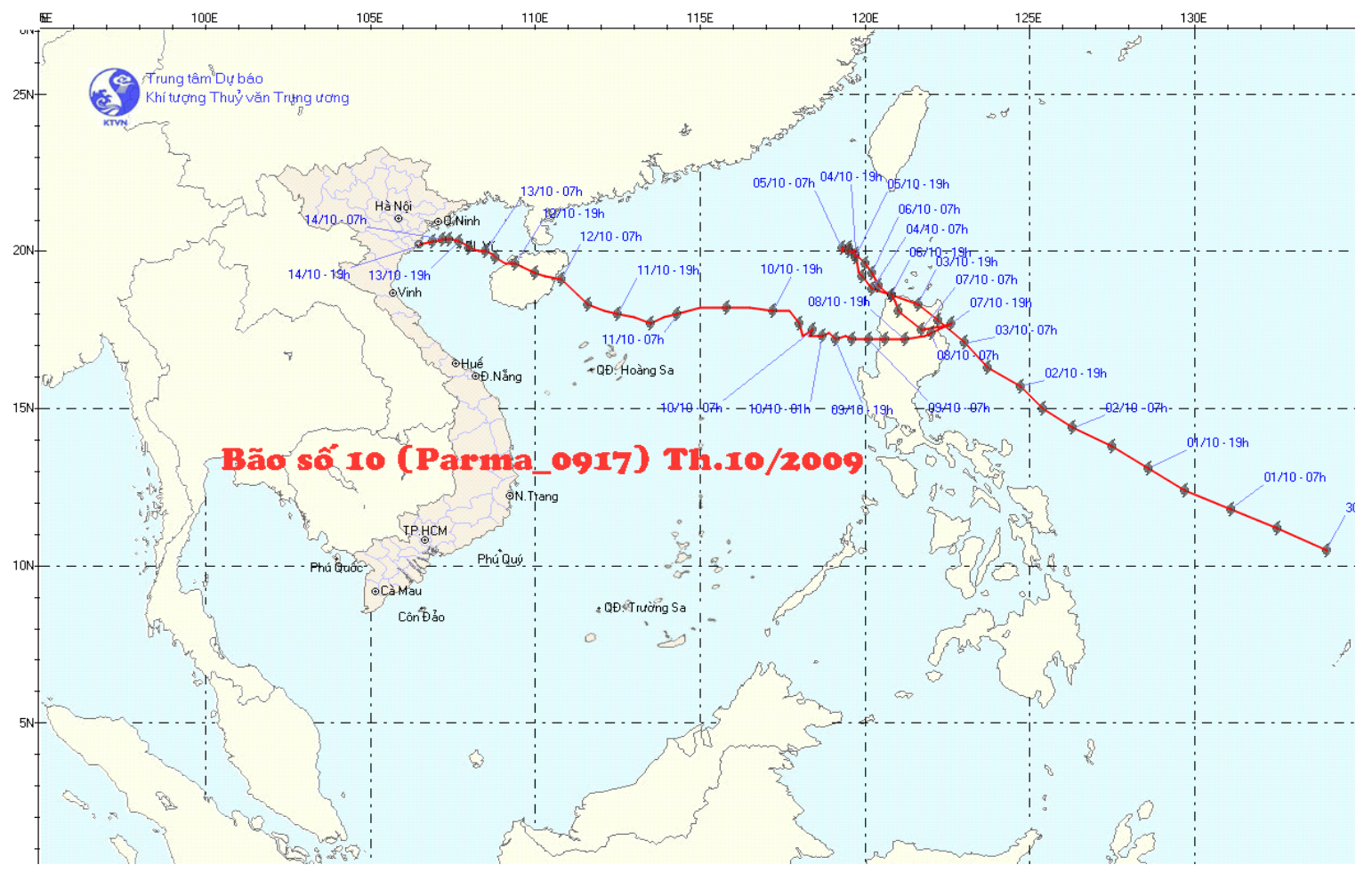

Figure 3.6 Track of the tropical storm PARMA in October 2009. Source: NCHMF, Vietnam.

After crossing the $122.0^{\circ}$ E longitude, PARMA came back to the SEAS region, crossed Luzon Island (Philippines) for the second time and re-entered SEAS on 9 October. PARMA was slightly intensifying while moving west and northwest to reach the Bacbo gulf. It dissipated over the coast of Thai-Binh - Nam-Dinh provinces on 14 October. This was one of the most complicated typhoon tracks in 2009 over SEAS. The maximum wind speed was observed at $42 \mathrm{~m} . \mathrm{s}^{-1}$, gusting to $53 \mathrm{~m} . \mathrm{s}^{-1}$ in Bach-Long-Vi station (WMO code \#48839, Chapter 
2). The minimum pressure of $989.0 \mathrm{mb}$ was recorded at $08.15 \mathrm{UTC} 13$ October in the \#48839 station. PARMA caused heavy rain in Quang-Ninh - Hai-Phong provinces over three days (13 - 15 October) with a total amount rainfall of $100-150 \mathrm{~mm}$, and even more at some stations such as: Mong-Cai (Quang-Ninh) 181mm, Bach-Long-Vi 193mm, Co-To 317mm, Thanh-Hoa 179mm.

\subsubsection{Drought}

The impact of drought affects the socio-economic status of the country, especially agriculture. In winter, from November to February, if the total amount of rainfall is less than $10 \mathrm{~mm}$ in any place, that place will be classified as a drought area. Similarly, $30 \mathrm{~mm}$ for the spring (March, April) and autumn (September, October), and 80mm for the summer (May to August) are criteria to identify drought areas in Vietnam. For example, every year PhanRangPhanThiet, a southern tip of coastal Central region, regularly witnesses severe drought from November-December to April. One of the possible drought mechanisms in that region is that during this period only the northeasterly winds prevails over a northeast-southwest topographical region, and there is no association of rain-producing system like tropical storm activated in the place.

Figure 3.7 shows the level of water in Red River, the major river in the North Delta, in Hanoi on 21 January, 2010. It was $0.48 \mathrm{~m}$, the lowest water level recorded in Hanoi in the last 100 years. Unfortunately, this station data only describes a part of the story of the very severe drought season in North Vietnam in the year 2009-2010. Serious drought occurred over a large area and lasted from August 2009 up to April 2010 in provinces in North and Central Vietnam. On average, the natural flow in most river basins was reduced by $30-90 \%$ in each consecutive month and was much lower than the annual average values compared with the same period in other years. 

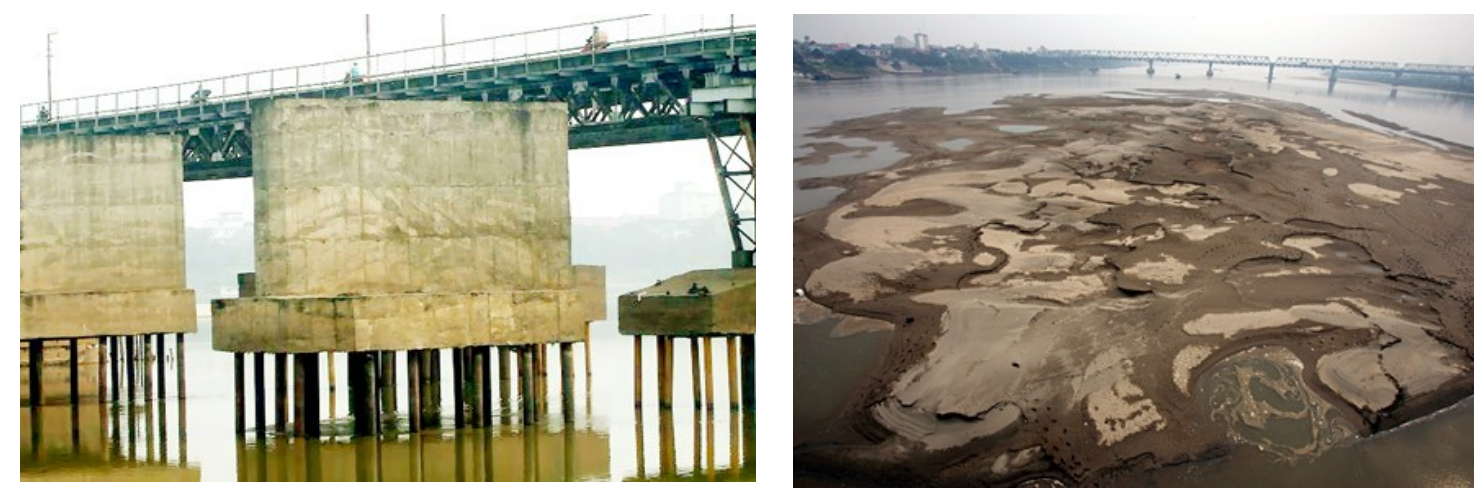

Figure 3.7 Breaking historical drought records in Red River on 21/01/2010. The water level was $0.48 \mathrm{~m}$, the lowest in the 100 years of observations. The Long-Bien bridge on that day in Hanoi.

\subsubsection{Flood}

Floods in Vietnam are usually the result of one of three main causes. The first source is from the natural torrential rainfall, the second source is from the dams ${ }^{1}$ water release, and the last source is the combination of these two sources. Most severe flooding areas extend from the North Delta to the Central, causing socio-economic damage and deaths. On average, there are about dozen massive floods observed in the country each year. For example, on 2-3 November 2009, due to the affect of the storm MIRINARE-0921 a heavy rainfall episode in the southern Central (Figure 3.8, 3.9), 200 - 300mm rainfall in 48h (2-3 November), was observed, causing a vast flooded area, about $150 \mathrm{~km}$ north-south long. More precipitation was recorded in other places such as Tra-My 391mm, Van-Canh 666mm, or Quy-Nhon (WMO code 48870) 368mm. A very intense rainfall, $294 \mathrm{~mm}$ in 6 hours, was recorded at Binh-Dinh province on the 2 November.

\footnotetext{
${ }^{1}$ The release of water from hydropower dams in Upper Mekong River in China may cause the downstream floods in Vietnam. Also, the release of water from reservoirs in upper basin provinces can cause floods to lower basin provinces
} 

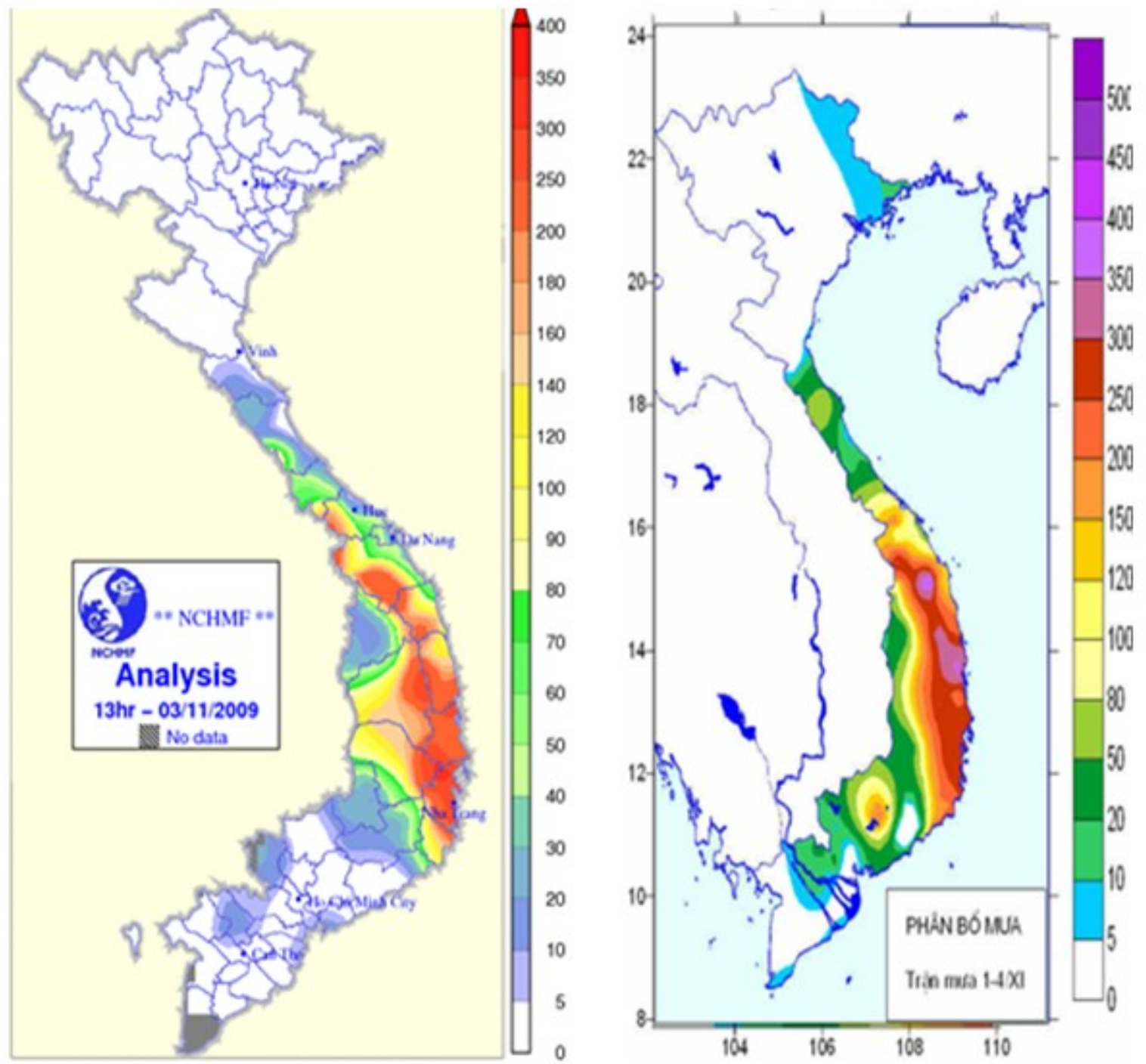

Figure 3.8. Maximum 24 hours accumulated rainfall from 06UTC 02 - 03 November 2009 (left), and the accumulated rainfall in the first four days in November 2009 (right). Unit: mm. 


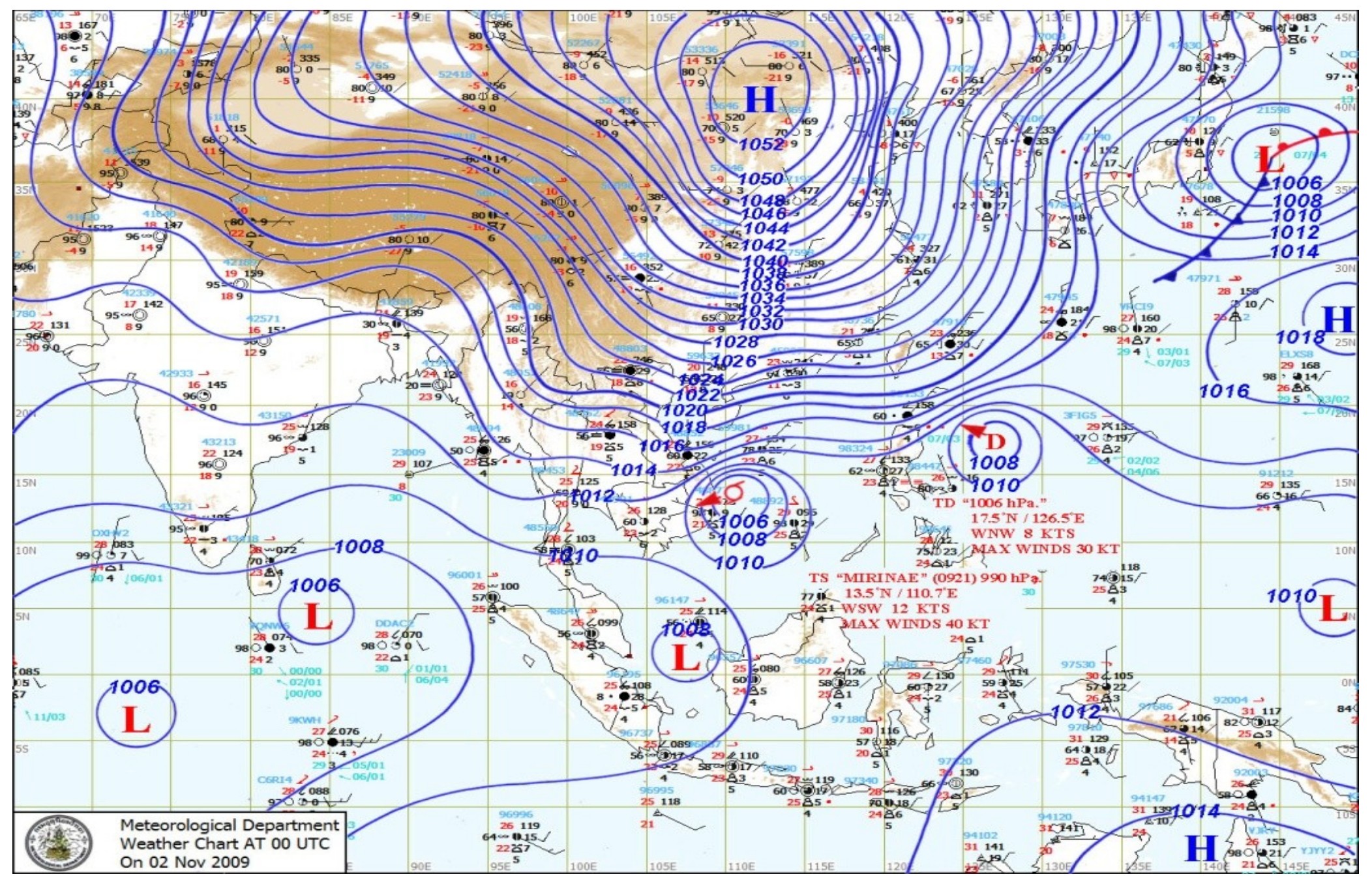

Figure 3.9. Surface analysis map on 00zUTC 02/11/2009, MIRINARE-0921 storm. Map is provided by TMD. 


\section{CHAPTER 4}

\section{CLIMATE VARIATIONS}

But Nature flies from the infinite, for the infinite is unending or imperfect, and Nature ever seeks an end.

\section{Aristotle}

This chapter characterises rainfall and temperature variability for the whole of Vietnam and for climate sub-regions over the forty years, 1971-2010. Vietnam's average temperature has increased at a rate of $0.26 \pm 0.10^{\circ} \mathrm{C}$ per decade since the $1970 \mathrm{~s}$, approximately twice the rate of global warming over the same period. The rate of increase is greater in winter than in summer. Except for the Central Highland, annual average temperatures in the southern regions are increasing more rapidly than in the North. The increases in temperature are statistically significant in most sub-regions; however this is not the case for rainfall. Temperature and rainfall variability are shown to be linked to ENSO on both a national and sub-regional scale. This relationship is stronger in lower latitudes and in winter. This chapter has mostly been presented in Nguyen et al. (2014a). 


\subsection{Climate in sub-regions}

The Vietnam sub-region climate was classified by Nguyen and Nguyen (2004). They classified the Vietnam climate into seven sub-regions, including the North (N1, N2, N3, N4) and the South (S1, S2, S3) (Chapter 3). They used the total amount of rainfall in rainy and dry seasons in stations to categorise these sub-regions. In their study, the data was up to the year 2000. Here, we revisited the Nguyen and Nguyen's (2004) sub-region climate classification by an objective classification method, including the more recent observational data, up to the year 2010 .

We use the K-means cluster analysis for the full anomalies data set (detail in Chapter 2). Figure 4.1 shows the six-to-nine sub-regions of anomalies temperature and rainfall. In general the K-means outcomes are consistent with Nguyen and Nguyen (2004), except at the farthest offshore island station. The rainfall and temperature anomalies of this $400 \mathrm{~km}$-offshore station, namely IS (Truong-Sa, $8.7 \circ \mathrm{N}, 111.9 \circ \mathrm{E}$ ), are compared to all other single stations as well as sub-regions, and they are statistically significant from continental sites (two-tailed t-test, $\mathrm{p}<0.001)$. 


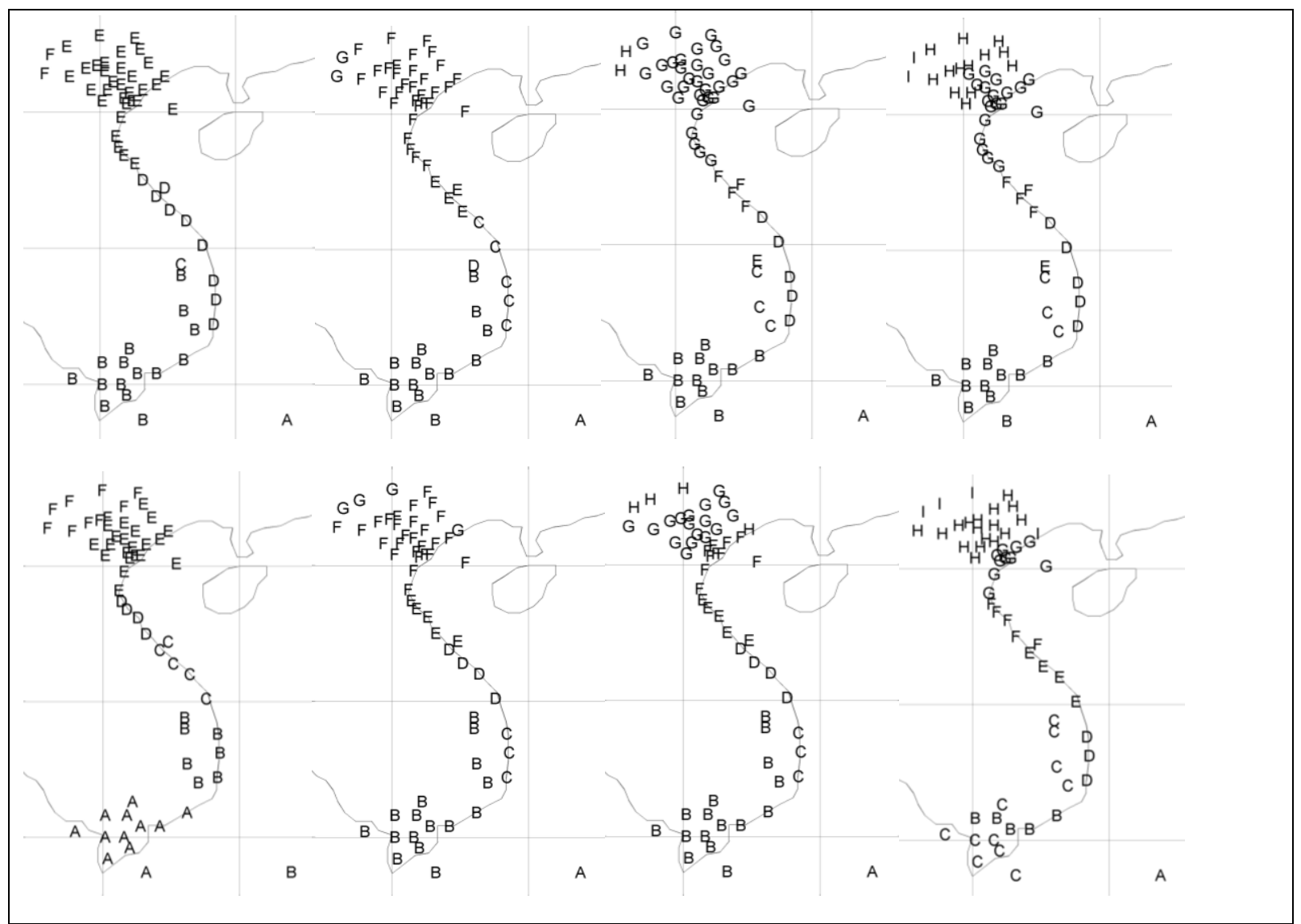

Figure 4.1. Cluster analysis for temperature anomalies (top) and rainfall anomalies (bottom) 19712010. From left to right: specification of 6,7,8,9 climate sub-regions, corresponding with $\mathrm{K}=6,7,8,9$ in K-methods. Alphabet letters are assigned to sub-regions. 
At the Vietnam Hydrometeorological Service (HMS), island stations were implicitly classified into the nearest sub-climate region on the continent. In this study the IS (Island Station), will be treated separately in all analyses. Hereafter, the Vietnam climate in this study is divided into eight sub-regions, consisting of seven continental sub-regions (Nguyen and Nguyen, 2004) and one offshore sub-region.

\subsection{Temperature variability}

\subsubsection{Average temperature}

In all Vietnamese climate sub-regions, temperatures have increased from 1971 to 2010, as observed in most other parts of the world (Jones et al. 2007). Out of the eight sub-regions, seven show a statistically significant trend $(\mathrm{p}<0.05$, Table 4.1). Mann-Kendall and Sen's methods are used for trend detection (Chapter 2).

\begin{tabular}{llll}
\hline & MJJA & DJFM & 12-months \\
\hline N1 & $0.24^{* * *} \pm 0.12$ & $0.30^{* * *} \pm 0.10$ & $0.25^{* * *} \pm 0.06$ \\
N2 & $0.20^{* *} \pm 0.11$ & $0.26^{* * *} \pm 0.14$ & $0.25^{* * *} \pm 0.07$ \\
N3 & $0.21^{* *} \pm 0.11$ & $0.28^{* *} \pm 0.13$ & $0.27^{* * *} \pm 0.08$ \\
N4 & $0.19^{* *} \pm 0.12$ & $0.23^{* *} \pm 0.14$ & $0.22^{* * *} \pm 0.11$ \\
S1 & $0.26^{* *} \pm 0.14$ & $0.44^{* * *} \pm 0.16$ & $0.35^{* * *} \pm 0.10$ \\
S2 & $0.24^{*} \pm 0.15$ & $0.29^{* *} \pm 0.17$ & $0.25^{* *} \pm 0.12$ \\
S3 & $0.28^{* *} \pm 0.16$ & $0.40^{* * *} \pm 0.21$ & $0.36^{* * *} \pm 0.13$ \\
IS & INS & $0.17^{+} \pm 0.17$ & $0.15^{+} \pm 0.13$ \\
VN & $0.23 \pm 0.16$ & $0.30 \pm 0.17$ & $0.26 \pm 0.10$
\end{tabular}

Table 4.1. Estimated trends in average temperature anomaly in Vietnam in the last 40 years. (Unit: ${ }^{\circ} \mathrm{C} / 10$ years). N1, N2,.., VN: climate sub-regions and the whole country. The asterisks (*) indicate the significant level p. $(* * *),(* *),(*),(+): 99.9 \%, 99 \%, 95 \%, 90 \%$ confidence interval for the existence of increased trend, respectively. 90\% $(\mathrm{p}<0.01)$ confidence interval of Sen's slope is in the range

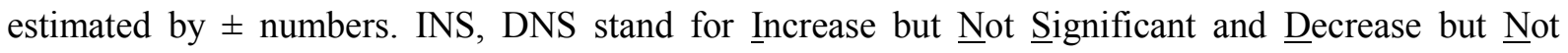
Significant, respectively. Trends for the whole country are calculated by the average of all sub-regions.

On average, surface temperatures in Vietnam have warmed at a rate of $0.26^{\circ} \mathrm{C} / \mathrm{decade}$ over the last forty years. This rate is approximately twice the average rate of global warming, which was estimated to be about $0.13 \pm 0.03^{\circ} \mathrm{C} /$ decade in the last fifty years (Jones et al., 2007), and slightly less than that of the global land-only in the Northern Hemisphere, which varies from 0.29 to $0.34^{\circ} \mathrm{C} / \mathrm{decade}$ (IPCC 2007, Ar4 3.2.2, Table 3.2). Other studies also 
recognise such fast warming rates at different places around the globe (Klingbjer and Moberg, 2003; Nandintsetseg et al., 2007; Santer et al., 2008; Collins et al., 2009; Martínez et al., 2010; Fan et al., 2011; Jhajharia and Singh, 2011). Temperature in southern regions (S1, S3) has increased faster than in the north (N1-N4). The warming rate in the region S2 is less than others in the south, possibly because of its largely high altitude nature (mostly 600-2000 m above sea-level). The rise in winter temperatures (evident since 1971) is around $25 \%-40 \%$ higher than that of summer over the same period. Hence, it is higher winter temperature that are principally responsible for the increase of Vietnam's average annual temperature over the period of this study.

The annual average temperature anomaly averaged across all stations in Vietnam has increased significantly since the late 1970s, consistent with JCDAS data (Figure 4.2). There is a high correlation coefficient $(\mathrm{r}=0.91)$ between observations and the JCDAS reanalyses. A difference between these two datasets of about $0.5^{\circ} \mathrm{C}$, increasing with time, is however apparent. The linear trends in the observations, the average of JCDAS grid-points over Vietnam's land mass, and over the selected JCDAS domain in Figure 4.2 are about 0.26, 0.10, and $0.12{ }^{\circ} \mathrm{C} /$ decade, respectively. 


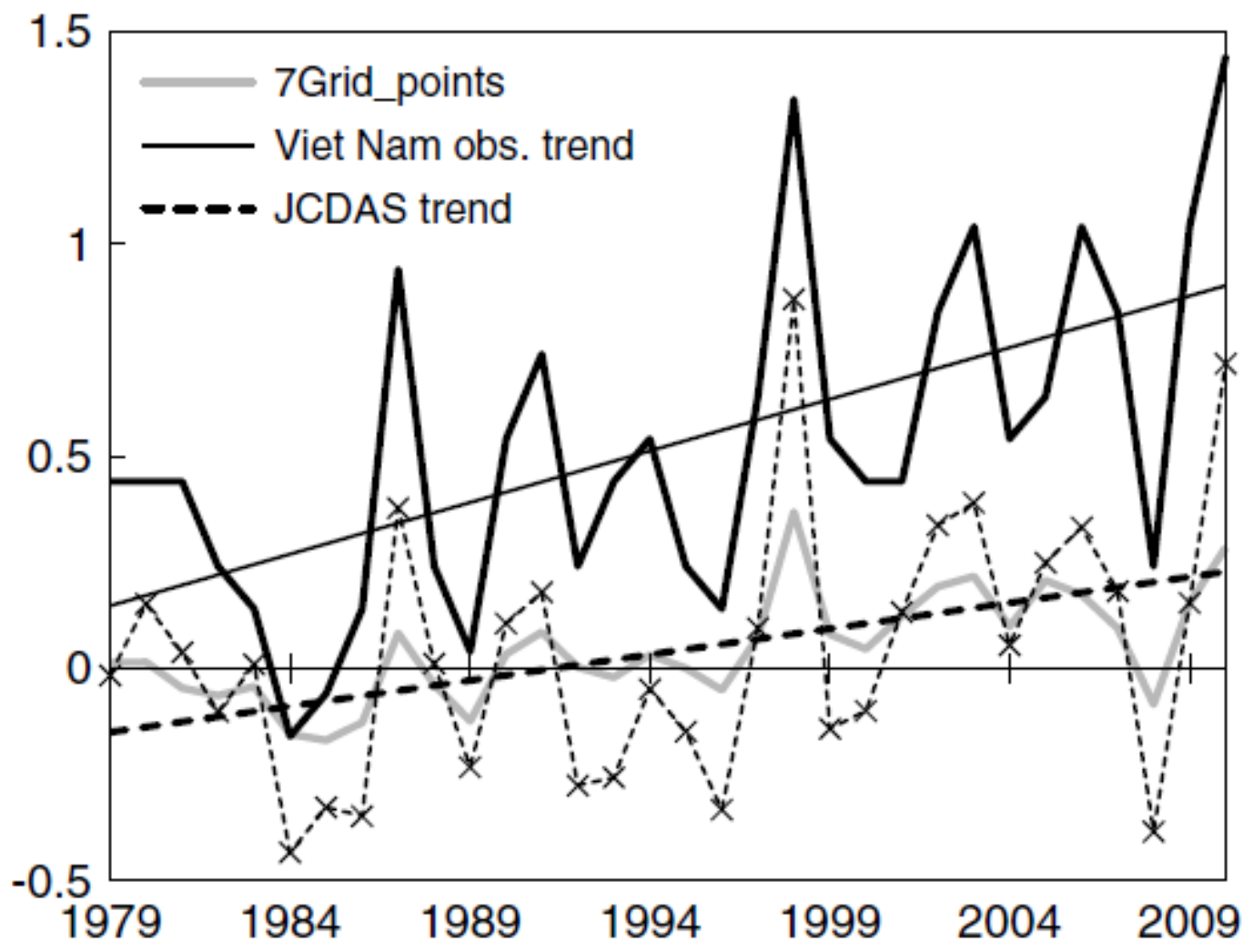

Figure 4.2. Variation of observation annual average temperature anomaly (black), JCDAS temperature anomaly averaged (dash) over domain (8-23N, 102-110E), and seven JCDAS gridded points (gray) over Vietnam.

This difference is explained partly because the selected JCDAS reanalyses domain, which covers a larger area than Vietnam, includes adjacent ocean regions. Another reason for this difference may be that fewer observational stations were used by JCDAS than the current analysis. In other words, "real" local atmospheric conditions were not represented in the JCDAS reanalyses data. A recent personal communication with the Tokyo Climate Center stated that fewer than ten stations are being updated regularly by Vietnam to this WMO Regional Climate Centre.

It is interesting to note that the annual average temperature in Vietnam has peaks in most ENSO warm phases, e.g. the years 1987-1988, 1997-1998, and 2009-2010. This is consistent with previous studies (Xie et al., 2003, Wang et al., 2006, Chowdary et al., 2012) although those studies did not specifically look at Vietnamese observations. This study investigates the time lag for sea surface temperature (SST) patterns from the Central Pacific to Vietnam, the 
eastern coastal region of the Indochina peninsula. We found that a four month-lag showed the highest Pearson correlations between temperature anomalies in Vietnam and the Niño3.4 index. This possibly indicates that ENSO can be best observed in Vietnam four months after an active ENSO phase appears in the Central Pacific. In general, for those sub-regions closer to equatorial latitudes, a more reliable relationship to ENSO was found (Figure 4.3).

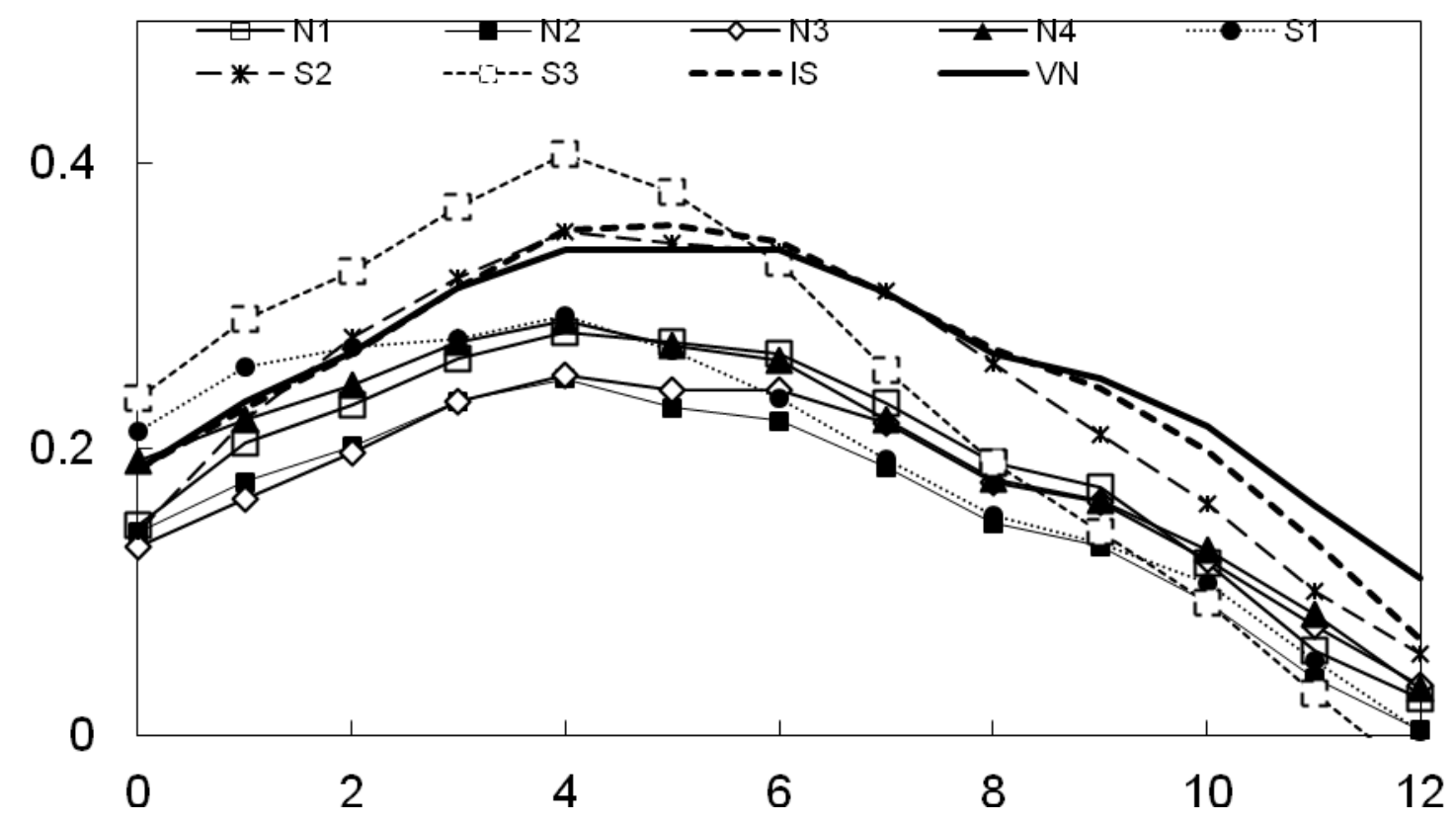

Figure 4.3. Pearson correlation coefficients between Niño 3.4 index and surface temperature in subregions in Vietnam. X-axis: month-lag, Y-axis: Pearson correlation coefficients.

Variations of temperature can also be investigated by the timing of summer and winter periods. In the different parts of the world, JJA and DJF are used for summer and winter respectively (for the Northern Hemisphere and vice versa in the Southern Hemisphere). However, for Vietnam we note that of the eight sub-regions, there are six regions in which the May-July (MJJ) average temperature is higher than that of the June-August (JJA); and there are seven regions in which the January-March (JFM) average temperature is lower than the December-February (DJF) average temperature (Figure 4.4). Thus, summer and winter in Vietnam have a long duration, MJJA for summer and DJFM for winter. 


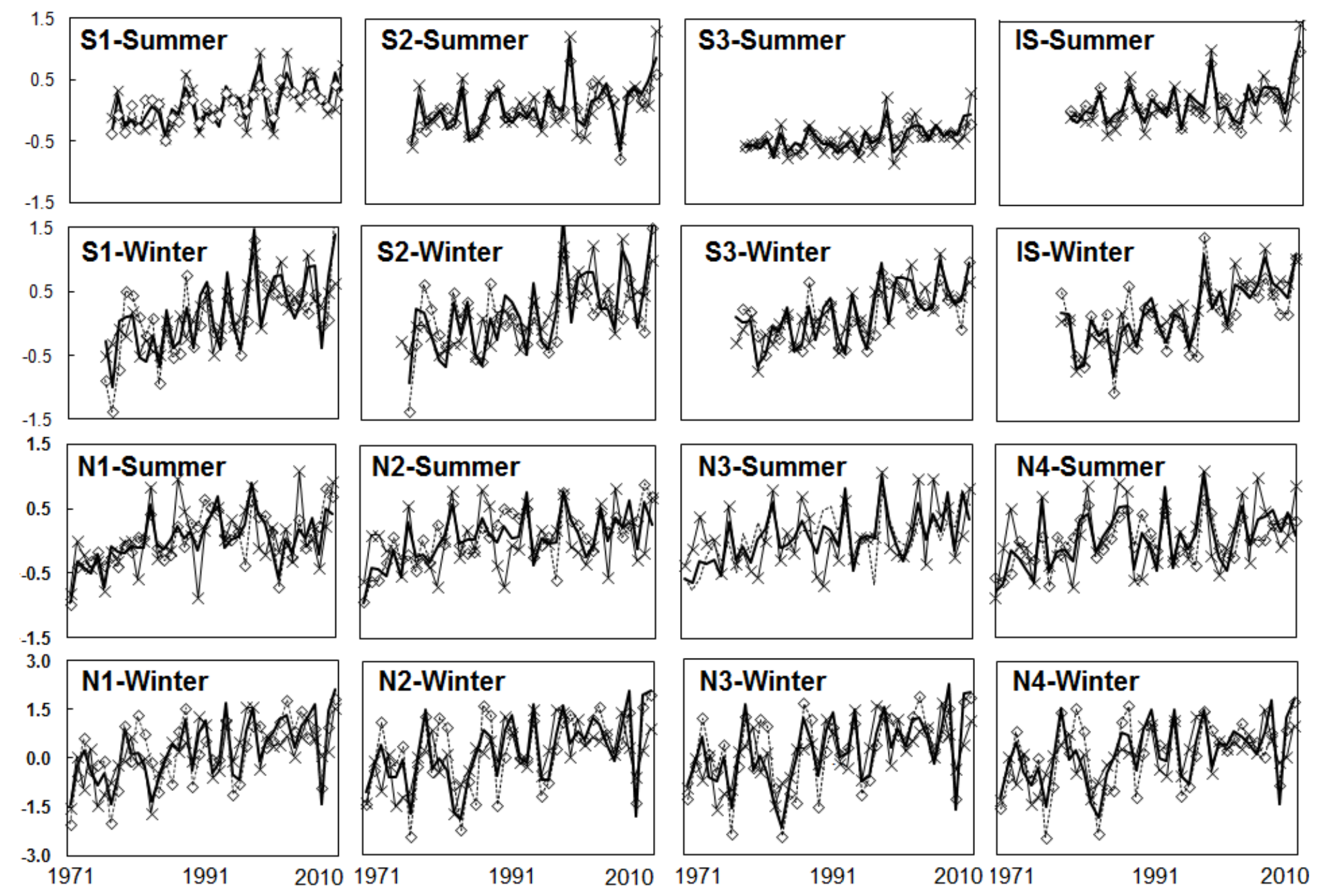

Figure 4.4. Summer and winter average temperature anomaly $\left({ }^{\circ} \mathrm{C}\right)$. Bold solid lines are the average anomaly JJA and DJF; thin lines are the average MJJ and NDJ; and dashed lines are the average JAS and JFM temperatures. 


\subsubsection{Maximum and minimum temperatures}

Since the daily maximum temperature (Tx) usually occurs in the day-time and the daily minimum temperature (Tn) occurs mostly at night, we consider that the change in Tx reflects changes in the larger-scale atmospheric state and the change in Tn represents changes in the local mesoscale state (Christy et al., 2009). Over the whole country the rate of increase of maximum and minimum temperatures varies wildly (Table 4.2). In the south, the rate of increase of Tn anomaly is greater than that of Tx anomaly. That means Tx and Tn in winter and summer both increased whereas the increment in winter is greater than in summer. In the IS, N1, and N3 the reversal occurs: the increment in maximum temperature in summer is greater than the minimum temperature in winter. Those temperatures in the N2 and N4 vary differently, but they are not statistically significant.

\begin{tabular}{|c|c|c|c|c|c|c|}
\hline & Tx an. & Tn an. & an.-Tn & Dry_ss & Rain_ss & \& Rainfall \\
\hline N1 & $0.18^{*} \pm 0.09$ & $0.12^{* * *} \pm 0.08$ & INS & DNS & DNS & DNS \\
\hline $\mathrm{N} 2$ & INS & INS & INS & DNS & DNS & DNS \\
\hline N3 & $0.21^{* * *} \pm 0.10$ & $0.12^{+} \pm 0.12$ & $0.11^{+} \pm 0.07$ & DNS & $-91.31^{*} \pm 53.12$ & $-84.40^{+} \pm 66.98$ \\
\hline N4 & INS & INS & DNS & $30.09^{* *} \pm 20.54$ & DNS & DNS \\
\hline S1 & DNS & $0.25^{* * *} \pm 0.15$ & $-0.19^{* * *} \pm 0.07$ & INS & $174.65^{+} \pm 144.06$ & INS \\
\hline $\mathrm{S} 2$ & $0.19^{+} \pm 0.17$ & $0.31^{* * *} \pm 0.16$ & $-0.21^{* * *} \pm 0.09$ & $91.34^{*} \pm 94.58$ & DNS & DNS \\
\hline S3 & $0.27^{*} \pm 0.17$ & $0.35^{* * *} \pm 0.15$ & $-0.13^{*} \pm 0.10$ & INS & INS & INS \\
\hline IS & $0.47^{* * * *} \pm 0.15$ & INS & $0.42^{* *} \pm 0.19$ & $127.71^{*} \pm$ & INS & $199.06^{* *} \pm 70.67$ \\
\hline
\end{tabular}

Table 4.2 Estimated trends in maximum, minimum temperature and rainfall. ( $\mathrm{Tx}$ an. $-\mathrm{Tn}$ an.): different temperature between average maximum temperature anomaly ( $\mathrm{Tx}$ an.) and average minimum temperature Tn anomaly (Tn an.). Dry_ss $=$ Rainfall in Dry Season, Rain_ss $=$ rainfall in Rainy season, and $\sum$ rainfall $=$ total rainfall over 12-month. Other abbreviations see Table 3.1. Unit for changes of rainfall: $\mathrm{mm} / 10 \mathrm{yrs}$.

The amplitude of Tx anomaly minus Tn anomaly (Tx-Tn) is decreasing in the south, but increasing in the north and at the Island (IS). Although Tx-Tn is affected by many different factors such as cloud cover, precipitation, vapour pressure (Dai and Trenberth, 1999; Dai et al., 2006), atmospheric circulation (Durre and Wallace, 2001; Wettstein and Mearns, 2002), greenhouse gases and aerosol (IPCC 2007, Ar4 3.2.2.7), cloud cover has usually been counted as one of the main causes (Dai and Trenberth, 1999). Our result could imply that there is a 
decreasing (increasing) trend of total cloud cover in the South (North and Island sub-region) which impacts more strongly on Tx rather than Tn. It is the significant increase of minimum temperatures that dominant influence the overall increase of temperature in the south. All the three southern sub-regions have a statistically significant trend. The increase of (Tx-Tn) in the north, however, is less significant. Only the sub-region N3, among the four northern subregions, has a non-zero increasing trend, at $90 \%$ confidence.

IS has the highest rate of change in (Tx-Tn). The changes in Tn anomaly are small, compared to that of $\mathrm{Tx}$, and are not statistically significant, therefore this high rate is mostly contributed by Tx. The IS average maximum temperature is increasing fastest among all subregions at $0.47 \pm 0.15^{\circ} \mathrm{C}$ per decade. The reasons for the high value at IS are not clear. Though this region is only represented by a single station, these results suggest that the warming over the ocean may be significantly higher than that over land in Southeast Asian region.

\subsection{Rainfall variability}

\subsubsection{Trends}

Rainfall variation, in general, has been studied in terms of four quantities: amount, intensity, frequency and type of precipitation (IPCC, 2013). The current study focuses on the first quantity, the total amount of rainfall in Vietnam.

Over the whole of Vietnam, dominant trends for annual rainfall show a decline, but evidently not statistically significant. Among the eight climate regions, five of them show decreasing trends but only N3 sub-region has statistically significant decreases. In S1, S3 and IS sub-regions there is an increase but only IS is significant (Table 4.2).

\subsubsection{Linkages to large scale forcing}

Lau et al. (2000) found connections between SSTA and Southeast Asian monsoon fluctuations. Two regional monsoon indices (one is the difference between the meridional wind at $850 \mathrm{hPa}$ and $200 \mathrm{hPa}$ and the other one is the difference between zonal winds at $200 \mathrm{hPa}$ ) have been correlated to SSTA in different seasons. Lau et al (2000) showed that SSTA patterns associated with monsoon indices have some resemblance to ENSO evolution. 
Nguyen et al. (2007) studied the relationship of monthly precipitation over Central Highland S2 and SSTA over tropical Pacific and Indian Ocean. For some months in the rainy season, such as April, October, and November, rainfall was highly correlated to ENSO. Based on heavy rainfall indices, Caesar et al. (2011) revealed that rainfall over the Southeast Asian Sea - a study region much greater than Vietnam and consisting of twelve Vietnamese stations has positive correlations to a La Niña-like SSTA pattern.

The EOF analysis has been used to determine the leading mode of variability of rainfall over the whole of the Indo-Pacific region, including Vietnam. The leading EOFs for the annual mean and for JJA and DJF (Figure 4.5) are similar in form and display ENSO-related variability (Dai et al. 1997; Dai and Wigley 2000). The second EOF modes vary seasonally but are also quite strongly ENSO-related. The time series of the EOFs shown are all correlated at around 0.5 with the SOI (averaged for the appropriate seasons). A correlation map between the SOI and the rainfall anomaly field is shown at Figure 4.6 (more detail in Appendix Figure AF4). The SOI correlations and the significant fractions of total rainfall variance accounted for by the leading pair of EOFs confirm that ENSO variability is a key determinant of Indo-Pacific rainfall, as reported by others (e.g. Dai and Wigley 2000). No clear pattern has been identified for the third leading mode of EOF analysis. 


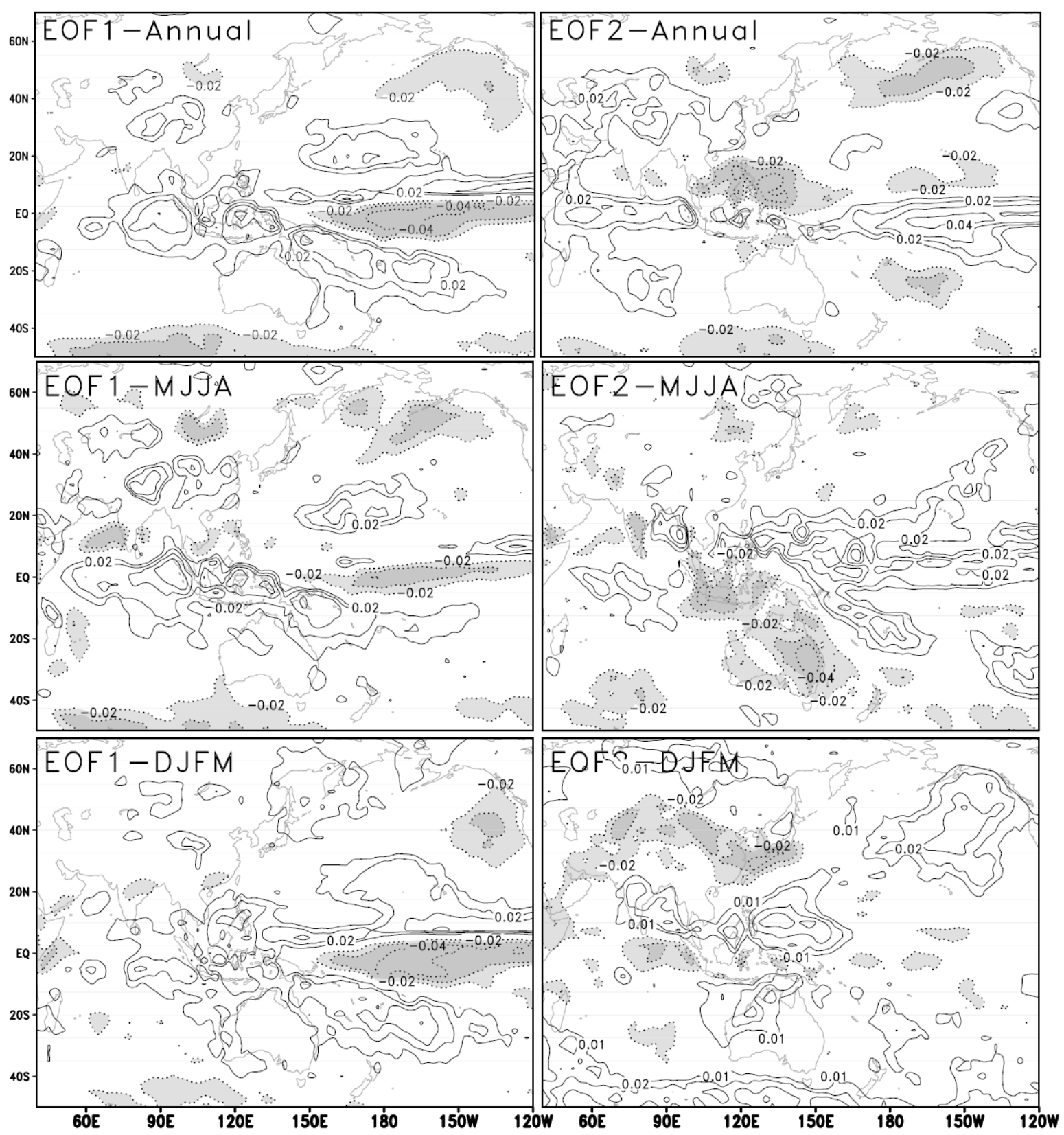

Figure 4.5. First two EOF components of JCDAS rainfall (mm/day) in Annual (top), MJJA (middle), and DJFM (bottom). Percentage variances explained by the leading modes are: Annual $(19 \%, 8 \%)$, MJJA (13\%, 7\%), DJFM (12\%, 8\%). Shaded areas show negative values. 

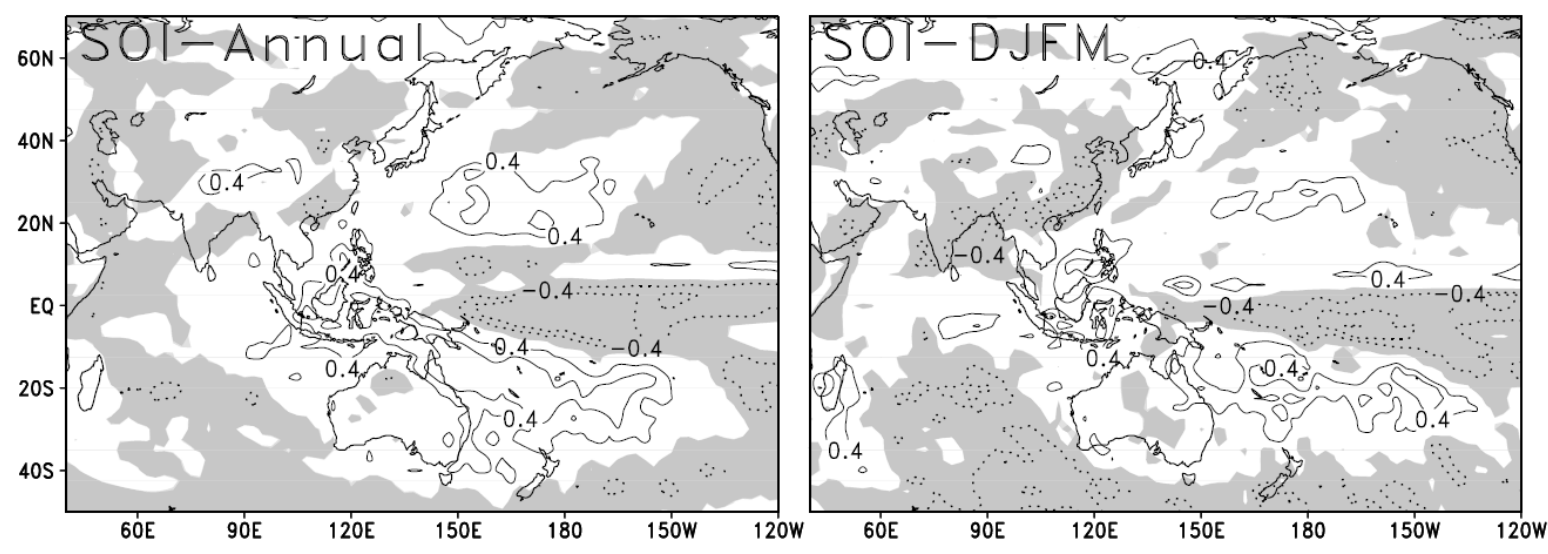

Figure 4.6. Correlation maps between annual, DJFM rainfall and the Southern Oscillation Index (SOI). Shaded areas show negative values. Only correlation coefficients significant at the $90 \%$ level are displayed.

The relationship between the large scale circulation and Vietnam rainfall in MJJA, DJFM, and 12-month averages was also investigated. Figure 4.7 shows correlation maps between Vietnam's rainfall and SST and MSLP. Correlation between SSTA and seasonal rainfall over Vietnam have ENSO-like pattern. Vietnam's rainfall is negatively correlated with SSTA variation in the central equatorial Pacific. Figure 4.7 (SSTA-DJFM) shows a look-alike LaNĩna pattern where SSTA in the Nino 3.4 region is negative and DJFM's rainfall over Vietnam enhances. The reverse pattern, shown in correlations between SSTA and MJJA rainfall (Figure 4.7, SSTA-MJJA), has a positive SSTA in Niño 3.4 region and a negative in rainfall over Vietnam, possibly representing the El-Niño characteristic: a strong dipole with a warm polarity over the Niño 3.4 region and a cold polarity being located at the Indo-Pacific region. Although a part of these correlation maps is in agreement with Wang et al. (2000) and Caesar et al. (2011), this is the first time the relationship between annual and seasonal rainfall over the Vietnam domain, a much more smaller domain compared to previous studies, and SSTA and Sea Level Pressure over the India-Pacific Ocean has been shown. In DJFM, when the Australia Low tends to be deeper in La Niña events, the pressure gradient between the Asiatic High in the Northern Hemisphere and the Australia Low in Southern Hemisphere increases, resulting in a strengthening northeasterly along the East Asian coast (Figure 4.7, MSLP-DJFM). This suggests that cold surges are more active during La Niña years, supporting Zhang et al. (1997) but is counter to the results of Chen (2002). 

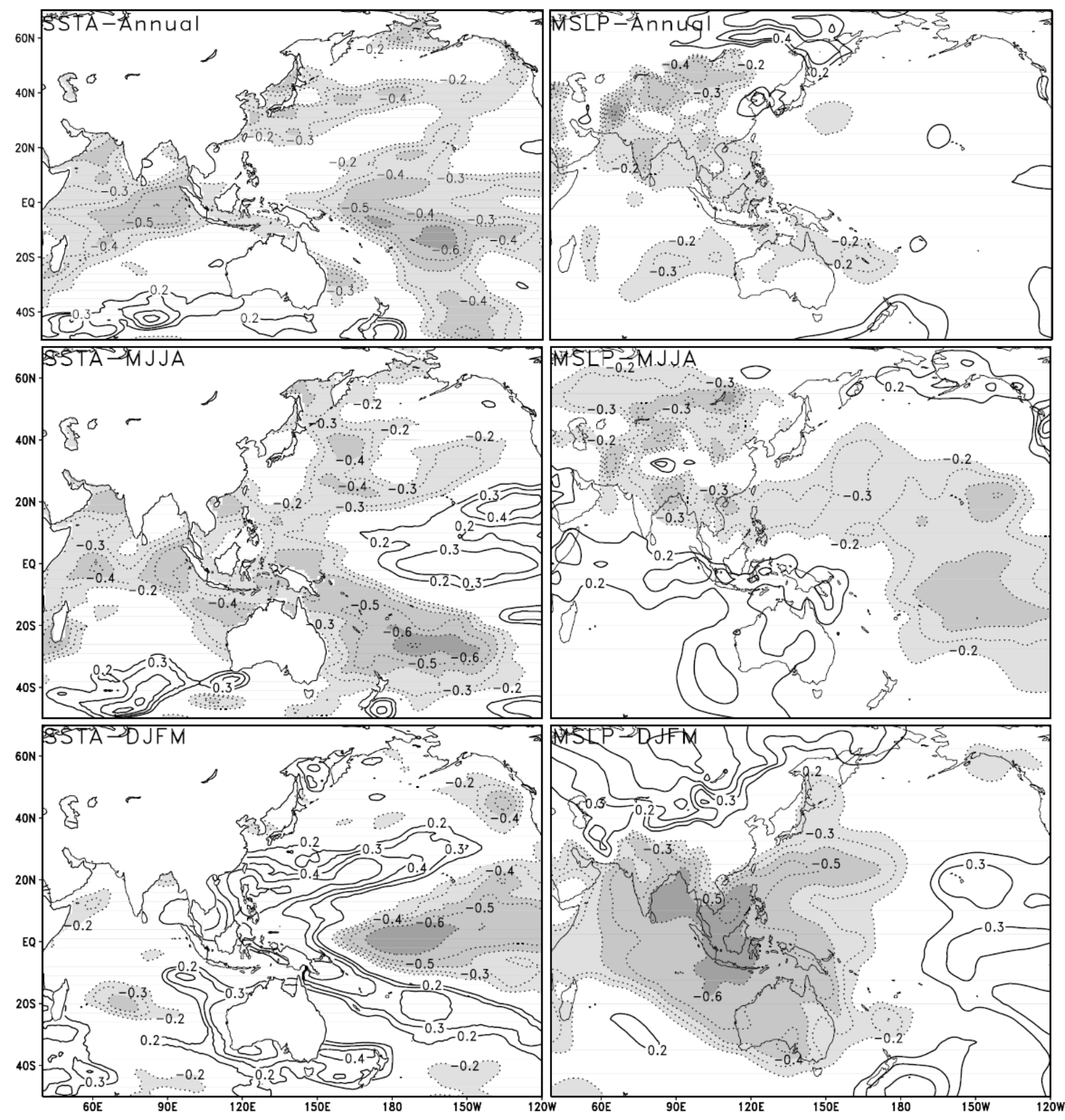

Figure 4.7. Correlation maps between SSTA and MSLP and annual rainfall (top), MJJA rainfall (middle), and DJFM rainfall (bottom). Only correlation coefficients significant at the $90 \%$ confidence level are displayed. Shaded areas show negative values. 
Spatial correlation maps between rainfall anomalies in each sub-region and SSTA and MSLP are constructed. For those stations in the north, ENSO signals can hardly be found (Appendix Figure AF5). Correlation maps between MSLP and southern sub-regions are shown in Figure 4.8. While S1, S3 and IS are sub-regions that show relatively strong ENSO signals (in both warm and cold events), S2 only shows the connections with ENSO in MJJA. S2's elevation condition (mostly 600-2000 m above sea-level) may affect its linkage ability. Rainfall is a complex process involving mechanisms such as topographical conditions, internal dynamics of the monsoon circulation, and external forcings; we have highlighted only the connection of rainfall in Vietnam and ENSO activity in this study. 

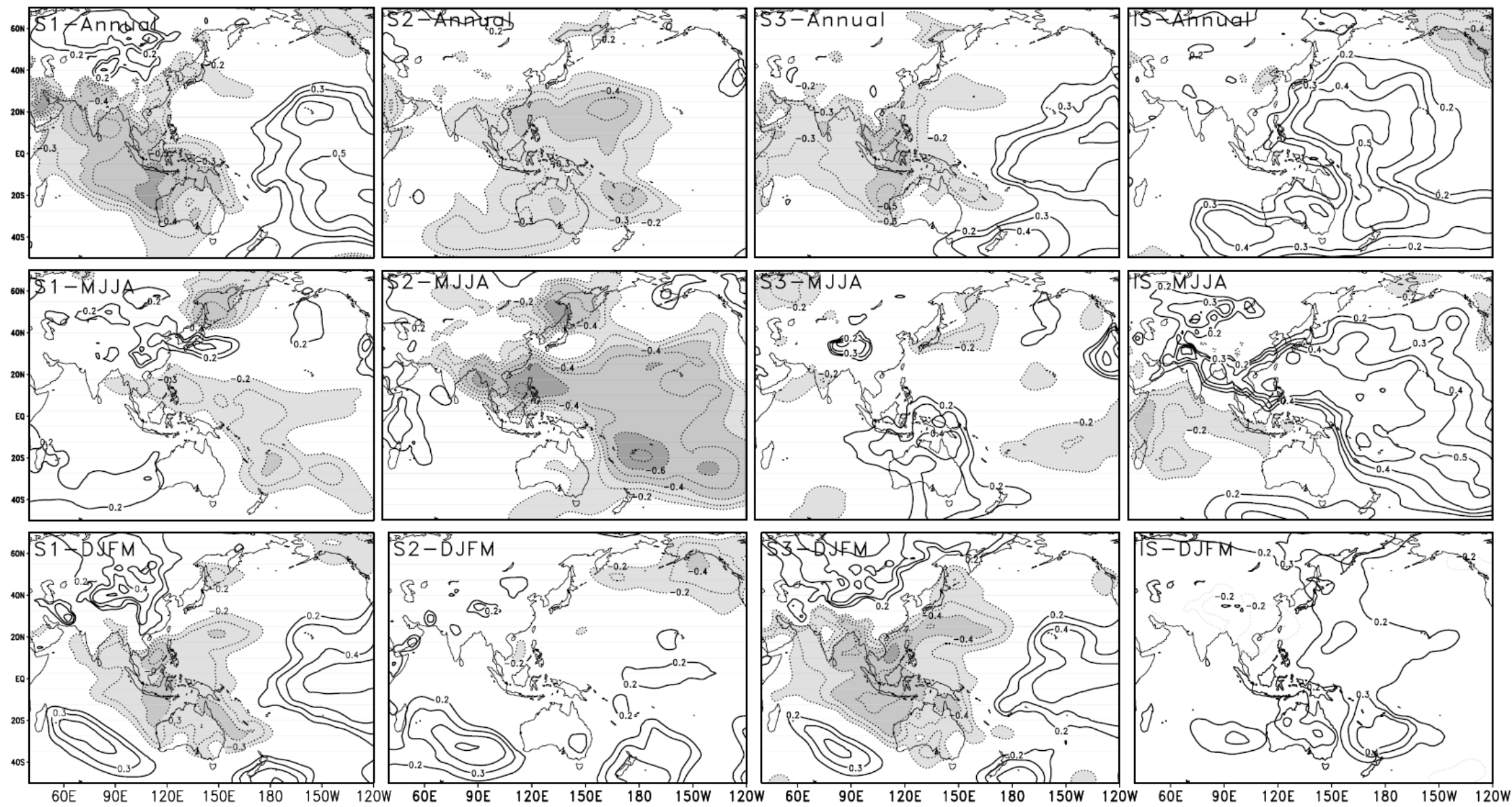

Figure 4.8. Correlation maps between MSLP in REG1 domain and Vietnam sub-region rainfall. From left to right: S1, S2, S3, IS sub-region. From top to bottom: Annual, MJJA, DJFM. Only correlation coefficients significant at the $90 \%$ confidence level are displayed. Shaded areas show negative values. Contour level: 0.1. 


\section{CHAPTER 5}

\section{MONSOON INDEX}

It can scarcely be denied that the supreme goal of all theory is to make the irreducible basic elements as simple and as few as possible without having to surrender the adequate representation of a single datum of experience.

Albert Einstein

One of the significant contributions of this Ph.D study is the development of a universally applicable index for determining the onset and withdrawal times of monsoons. Mean sea level pressure and low level zonal wind are combined to establish a standardised monsoon index. The index is able to determine the timing of the summer monsoon rainy season across all major monsoon regions in Asia-Australia, Africa, and the Americas. The index shows no sensibility to the choice of time and domain. The detailed application of the index in detecting the monsoon onset-withdrawal phases is demonstrated for the Vietnam region. Another important application of the monsoon index is the discrimination of monsoon and nonmonsoon rainfall. This discrimination provides a better opportunity to understand the monsoon and non-monsoon rainfall in terms of variation and predictability. Most of this work is published in Nguyen et al. (2014b). 


\subsection{Monsoon features}

Monthly mean rainfall at several stations around the globe is presented in Figure 5.1 below.
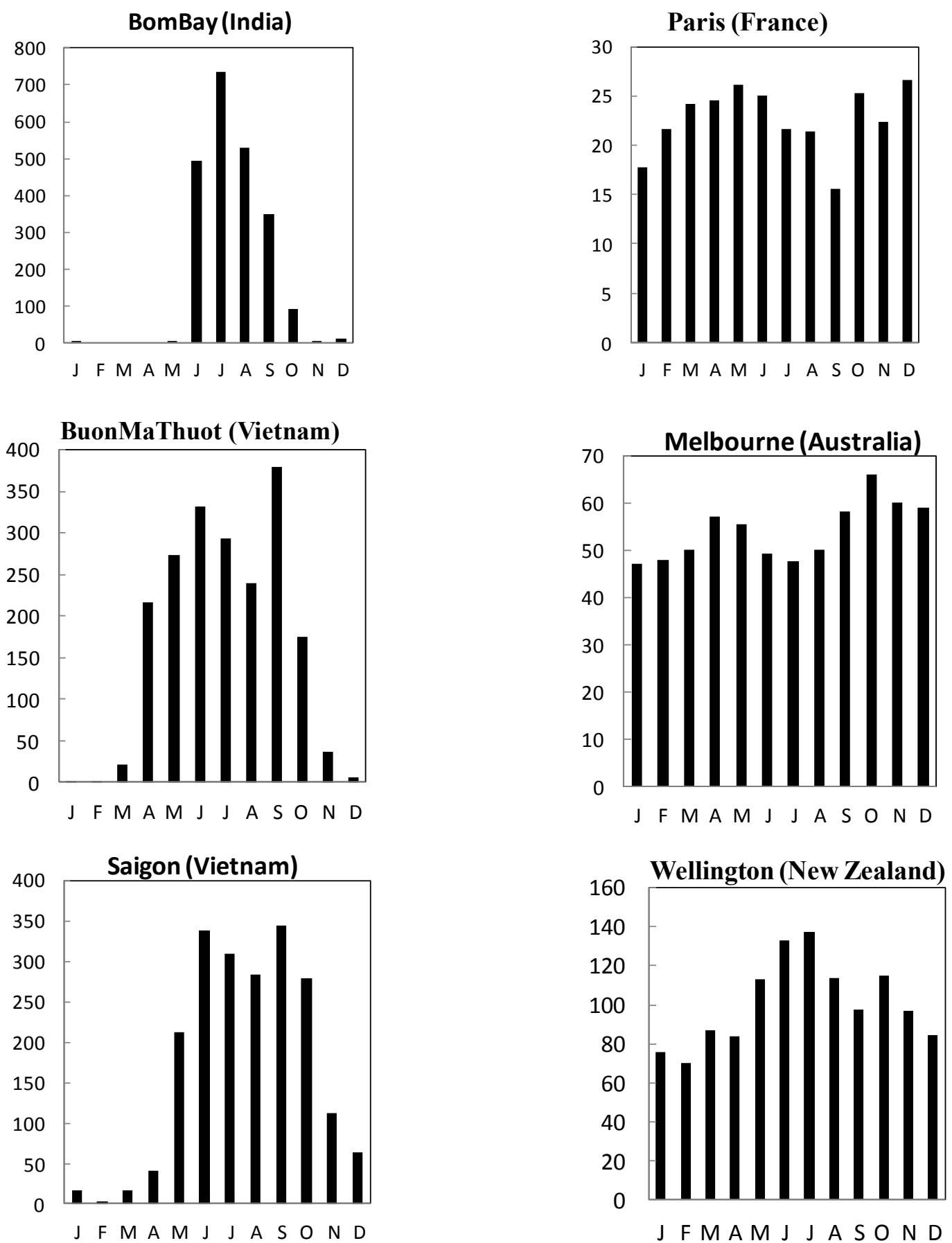

Figure 5.1 Monthly mean rainfall at some stations around the world. Data for BuonMaThuot and Saigon stations are derived from Bruzon and Carton (1930); other data from the corresponding National Meteorological Services for the period (1960-1990). Unit:mm. 
For those stations in monsoon regions (Bombay, Saigon, Buon-Ma-Thuot), an abrupt increase of rainfall is observed at the beginning of rainy season. Thus, identifying the monsoon onset date (OD) is a crucial step in studies of interannual variability of monsoon evolution. There are differences in the estimation of the specific OD between the various studies, since different criteria were used to define it. For example, the average OD for the Southeast Asian Sea (SEAS) region is estimated to occur from the end of April to mid-May (Qian and Lee, 2000), 9 May (Zhang et al., 2004), 12 May (Pham et al., 2010), or 14-19 May (Mao and Chan, 2004). In general, criteria can be classified into four types: rainfall and its proxy such as out-going longwave radiation (OLR) (Qian and Lee, 2000, Zhang et al., 2004), low-level wind (Wang et al., 2004), temperature (Li and Yanai, 1996; Mao et Chen, 2004), and a combination of low-level wind and rainfall and/or its proxy (Kueh and Lin, 2010; Pham et al., 2010). Wang et al. (2008) listed 25 different indices for OD identification; this list has been widening since then. This list, on the one hand, described the complexity of relationships associated with monsoon onset dates (OD); on the other hand it suggested that there is more than one solution for the OD identification.

Monsoon withdrawal date (WD) is the counterpart of OD as the combination of OD and WD determining the length of the rainy season. In our understanding, only a few studies have investigated WD. After withdrawal days, the prevailing southwesterly starts to change into the winter northeasterly. This northeasterly remains until a new summer monsoon cycle returning in the following year. Therefore, the role of WD is as considerably important as the OD.

The monsoon circulations, associated with seasonal reversals of winds over major tropical land masses, dominate the seasonal cycle of precipitation in the tropics. Thus, a monsoon index, if any, should in general include consideration of the seasonal change of rainfall and associated changes in prevailing winds. Most previous attempts to establish a monsoon index have been limited to specific regions and accordingly have lacked the desirable property of universal applicability. Recognising this shortcoming Li and Zeng (2002, hereafter LZ02) proposed a unified monsoon index, which they claimed was suitable for all monsoon regions. LZ02 demonstrated success in describing seasonal and interannual variability, but as this index was based on normalised monthly winds, it was unable to determine the precise timing of the onset/withdrawal of monsoons. By using universal criteria Zhang and Wang (2008, hereafter ZW08), introduced a monsoon index which identified the rainy season and was able 
to determine onset/withdrawal times for most monsoons. However, ZW08 noted that monsoon cycles in some "spots" could not be detected. This inability was attributed to their use of a fixed rainfall threshold.

The monsoon is essentially a rainfall phenomenon, but since rainfall is intermittent in space and time, we seek proxies that can characterise rainfall in terms of smoothly-varying large-scale parameters. In this chapter we introduce a simple two-component index for the study of monsoon variability to capture the two main features of the monsoon, rainfall and wind.

\subsection{Monsoon index definition}

\subsubsection{The two components}

To seek a good proxy for rainfall, SVD analysis is performed by diagonalising the covariance matrix between the rainfall and each of the other variables in turn. All fields are defined for the globe, within $40^{\circ}$ latitude of the Equator, on a regular $2.5 \times 2.5$ degree latitude/longitude grid. Table 5.1 lists the statistics of the first three SVD patterns for each variable, including the percentage of squared covariance accounted for, the variance accounted for in the possible proxy variables, the temporal correlations between pairs of expansion coefficients, and the spatial correlations between the paired SVD patterns.

\begin{tabular}{lllll}
\hline & Squared & Variance of Proxy & Temporal & Negative \\
\hline R- & $48(12)(6)$ & $17(9)(12)$ & $0.9(0.8)(0.7)$ & $-0.4(-0.5)(-0.2)$ \\
\hline R-OLR & $36(12)(7)$ & $8(4)(3)$ & $0.9(0.9)(0.9)$ & $-0.9(-0.9)(-0.9)$ \\
\hline R-U200 & $14(7)(6)$ & $15(0.1)(0.1)$ & $0.9(0.3)(0.3)$ & $-0.4(-0.01)(-0.01)$ \\
\hline R-H850 & $44(13)(9)$ & $18(13)(9)$ & $0.8(0.7)(0.7)$ & $-0.3(-0.4)(-0.5)$ \\
\hline & Squared & Variance of Proxy & Temporal & Positive \\
\hline R-U850 & $32(12)(7)$ & $8(5)(4)$ & $0.9(0.9)(0.9)$ & $0.4(0.3)(0.3)$ \\
\hline R- & $28(10)(7)$ & $5(3)(2)$ & $0.9(0.9)(0.9)$ & $0.9(0.9)(0.9)$ \\
\hline R-T2M & $40(21)(6)$ & $11(12)(4)$ & $0.9(0.8)(0.8)$ & $0.2(0.1)(0.4)$ \\
\hline R-T850 & $39(16)(7)$ & $10(10)(4)$ & $0.9(0.8)(0.9)$ & $0.3(0.2)(0.5)$ \\
\hline
\end{tabular}

Table 5.1. Statistics of the leading SVD pairs of global monthly anomalies of rainfall and 8 variables (MSLP, U850, convective rainfall (CONV), 2-m temperature, out-going long-wave radiation (OLR), zonal wind at $200 \mathrm{hPa}$ (U200), temperature and geopotential height at $850 \mathrm{hPa}$ (T850, H850)) during 32 years (1979-2010). The first(second)(third) leading mode are separated by parentheses. Covariance and variance unit: \%. The cumulative variance of rainfall accounted for by the leading three patterns is about $10 \%$ in each analysis. 
There are two groups of proxy variables, identified by the sign of spatial correlations with the rainfall patterns.

The first group of variables includes MSLP, OLR, U200, and H850 (negative spatial correlations with rainfall patterns); the second group consists of the rest of the proxy variables (U850, convective rainfall, $\mathrm{T} 2 \mathrm{~m}$, and $\mathrm{T} 850$, positive spatial correlations with rainfall patterns). The objective monsoon index is a combination of two representative variables, one from each group. For the first group, MSLP is selected because of its superiority in all statistical analyses. The three leading MSLP modes account for $66 \%$ of the squared covariance (the highest score in the group) between the two fields, and $38 \%$ of the MSLP variance (the second highest), and have the second highest temporal correlations. The first MSLP pattern itself explains $17 \%$ of the total MSLP variance. This pattern is characterised by an east-west seesaw at all tropical latitudes, and changes the sign near the dateline (Figure 5.2a). Figure 5.2c shows the regression pattern for MSLP, rainfall, and winds at $850 \mathrm{hPa}$ based on the MSLP time series from SVD pattern 1 (represents the average anomalies associated with $\mathrm{a}+1$ standard deviation departure of the MSLP time series). The rainfall pattern here is similar in form to the patterns of correlation and regression of the Tahiti-Darwin SOI and rainfall anomalies shown in Trenberth and Caron (2000a).

In the second group, temperature or zonal wind at $850 \mathrm{hPa}$ could be selected, based on their statistical characteristics (Table 5.1). From consideration of dynamical features of the monsoon cycle, U850 was chosen over T850. The first three patterns for U850 account for $51 \%$ of the squared covariance with rainfall, explain $17 \%$ of the variance of the U850 field, and show the second highest temporal correlations in the group. The dominant pattern of westerlies and easterlies can strongly impact rainfall activity in the tropics. Figure 5.2d presents the regression of the $850 \mathrm{hPa}$ winds, rainfall, and SST against the leading U850 time series from the SVD analysis. In the polarity shown here, the main features over the entire Pacific Ocean are consistent with typical La-Niña characteristics: strong easterlies, cool waters in equatorial eastern and central Pacific, and an enhanced rainfall centre around $120^{\circ} \mathrm{E}$. The second SVD patterns of both MSLP and U850 with rainfall are also related to ENSO.

The two components are chosen by the lead statistical characteristics with the regards of physical understandings. The index, therefore, is not sensible to the selection process. 

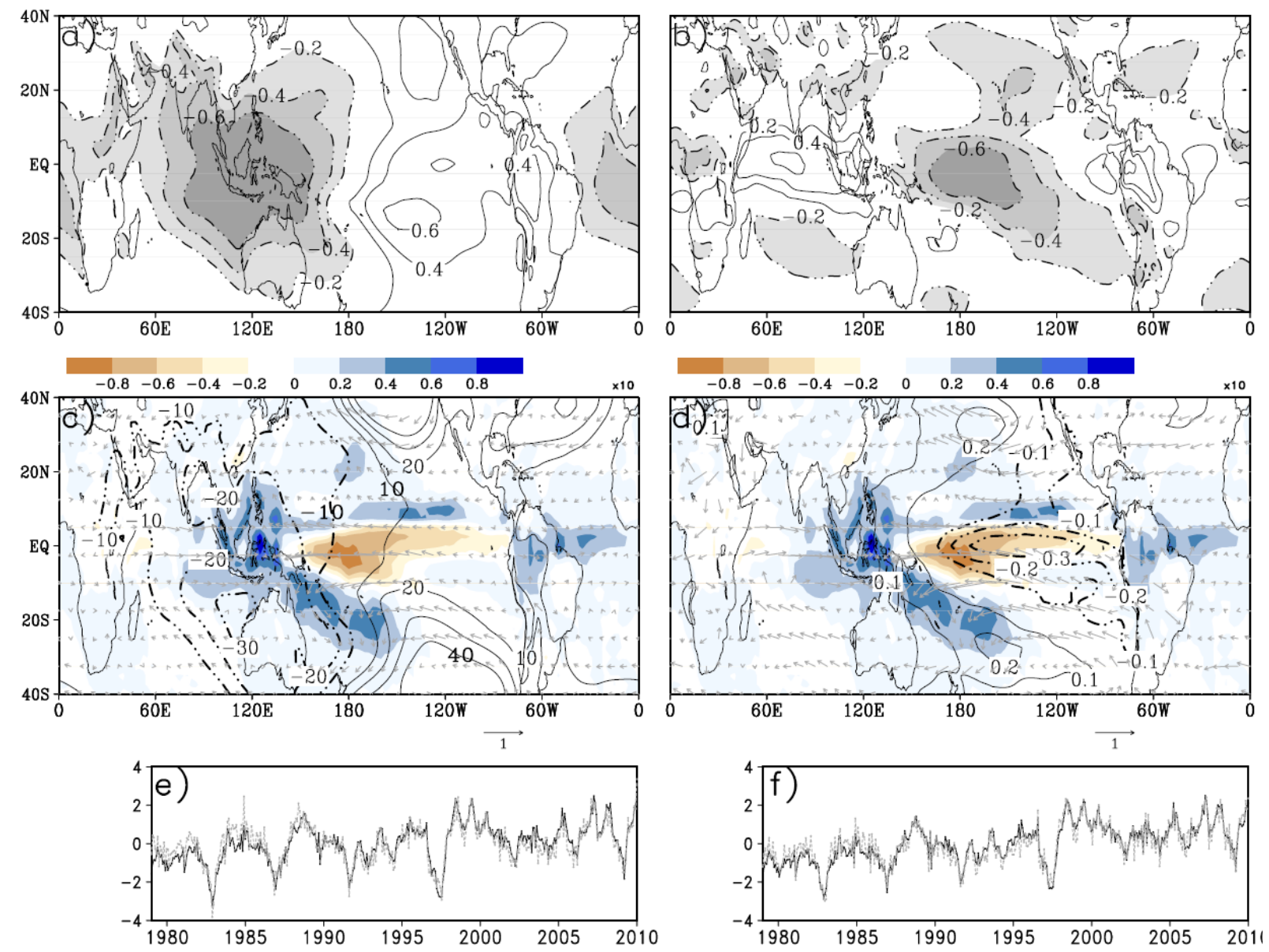

Figure 5.2. a-b) Heterogeneous correlations of SVD1 of global monsoon rainfall and MSLP(left) and U850(right) using all calendar months, 1979-2010; c-d) Regressions of rainfall (shaded, mm/day), MSLP(left, contours, Pa), SSTA(right, contours, $\operatorname{degC}$ ), and U850(vectors, m/s) onto the SVD1 proxy variable expansion coefficients (MSLP, left; U850, right); e-f) SVD1 timeseries of rainfall (solid, black) and e) MSLP(left, gray) and f) U850 (right, gray). Y-axes in e-f) are standardized by deviations, X-axis: $1979-2010$.

Both MSLP and U850 are significant proxies of rainfall in the tropics which is consistent with previous studies. For instance, numerous studies have highlighted the large-scale characteristics and predictability of MSLP (Walker and Bliss, 1932; Meehl, 1987; Allan and Ansell, 2006; Lavers et al., 2013), and the dynamical features of monsoon systems was represented by U850 in Wang et al (2004) and Pham et al. (2010). 


\subsubsection{The index calculation}

MSLP and U850 were standardised by their mean and standard deviations over specific monsoon regions. The standardised MSLP (U850) follows a U (inverted-U) shape curve (e.g., Figure 5.3). The two crossover points between these non-dimensional indices determine the monsoon onset and withdrawal dates. Figure 5.3 is an example demonstrating the application of the monsoon index for the REG2 region.

The mean summer monsoon onset days occur on the first weeks of May, and their counterpart, the mean summer monsoon withdrawal days, appear around the end of September - beginning of October. This is in agreement with the general understanding of the rainfall season over Vietnam (Nguyen et al., 2014a). The final stage is to form a single monsoon index from these standardized indices of MSLP and U850. The monsoon index, named NRM (Nguyen, Renwick, McGregor), is created by multiplying the two time series, but taking account only of the sign of the U850 series. That is:

$$
\mathrm{NRM}=\operatorname{sign}(\mathrm{U} 850) \times \operatorname{abs}(\mathrm{MSLP} \times \mathrm{U} 850)
$$

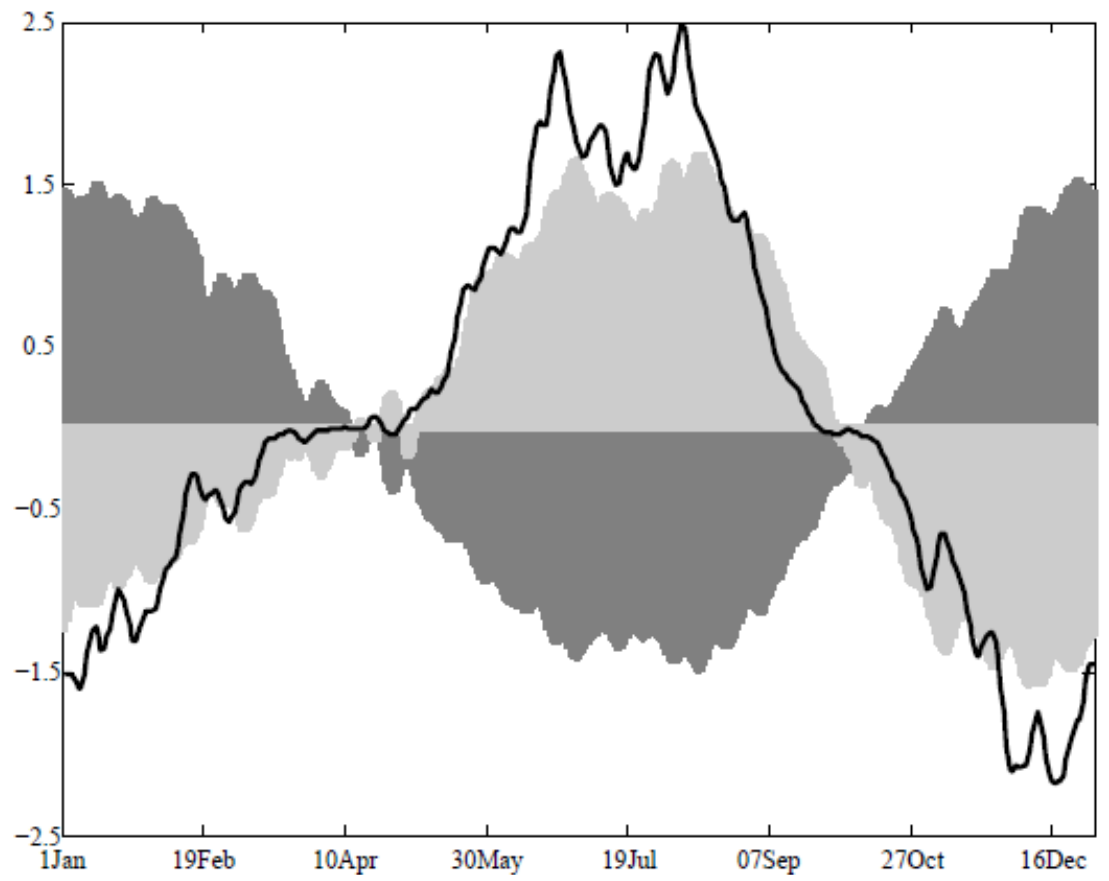

Figure 5.3. Mean evolution of MSLP (dark gray shaded), U850 (light gray shaded), and the monsoon index NRM (bold black) for 1979-2010 over the REG2. Y-axis: standardized anomaly, $\mathrm{X}$-axis: 5-day moving average from daily datasets. 
Since Figure 5.3 is smoothed by the 32-year average, the pattern of MSLP, U850, and NRM in each year can be expected to differ significantly.

In reality, the standardised MSLP and U850 index are usually to be crossed over more than twice a year. For instance, in 1989 the MSLP and U850 were meeting at least six times, such as 15 February, 1 April, 14 April, 17 May, 15 July, and 20 September (Figure 5.4). The year 1989 is one of the most complicated years in 1979-2010 in terms of identifying the OD-WD by NRM approach. An algorithm must be defined to ensure a unique monsoon period is defined in each year.

\subsubsection{The NRM algorithm}

First, the NRM index is categorised into a binary series of ones and zeroes where one and zero stands for positive and negative NRM, respectively. The result is, for example, the NRM from 01 January to 15 February containing all zeros, while the NRM from 15 July to 20 September, in contrast, being a series of all ones. Second, the principal rainy period is defined as the maximal-length series of ones. For the year 1989, the principal period would be 15 July

- 20 September. Given the fact that over monsoon regions the "break/active" monsoon episodes are observed, here in this study, we propose a 5-day and 75 percent rule (hereafter the 5-75 rule) to identify the length of summer monsoon rainy season. Break and active episodes are represented by sets of zeros (negative NRM) and ones (positive NRM), respectively. The rule states a monsoon rainy season can contain break episodes so that each break period allows no more than five consecutive days and/or the difference between the maximum values of NRM in two consecutive active episodes being less than $75 \%$ the maximal NRM in the principal period. Last, the summer monsoon rainy season is defined as the maximum period of positive values of NRM with the 5-75 rule consideration. OD and WD are determined as the first and last days of that positive period. Thus, the 1989 OD (WD) over REG2 is 14 April (20 September), in association with the two breaks, 12-16 May and 1114 July 1989.

The proposed "5-75" rule is an empirical rule which is based on experiments in different monsoon regions. Since the "break/active" monsoon episodes are different from this region to 
the other region, applying a same rule to all monsoon regions possibly result in some uncertainties. However, the " $5-75$ " rule so far has shown its satisfaction globally.

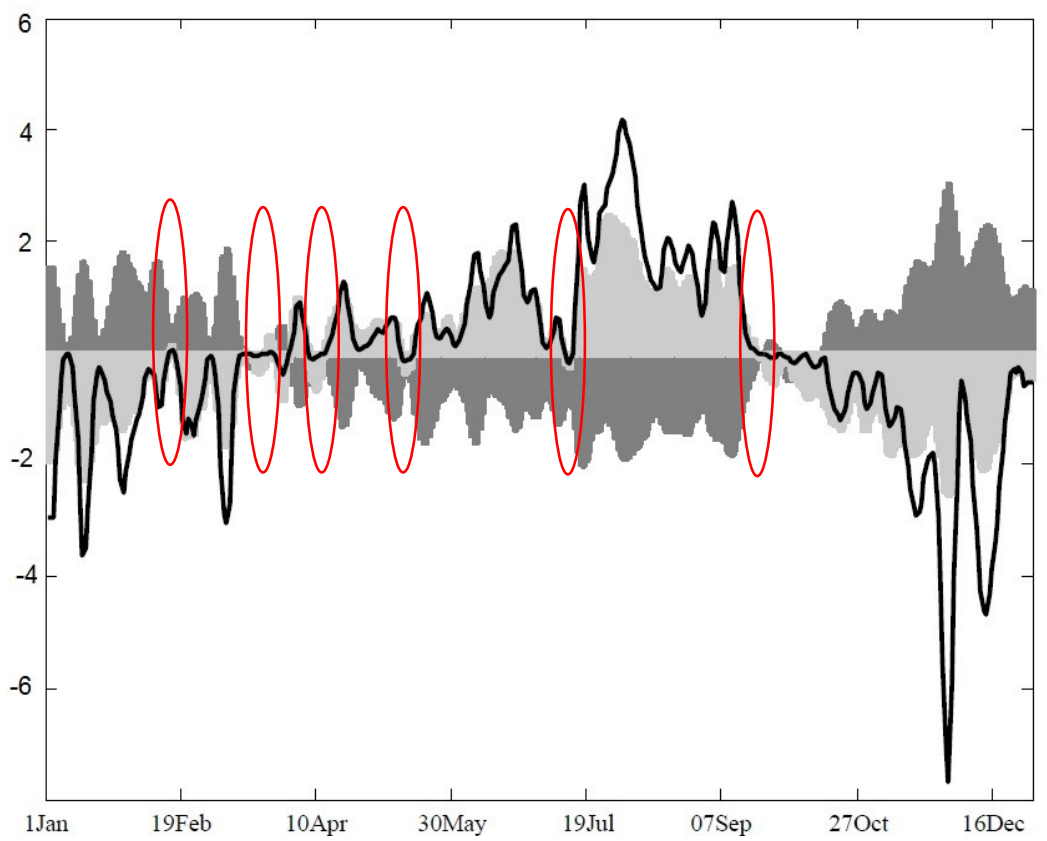

Figure 5.4. The same as Figure5.2, but for the year 1989. Red circles from left to right represent crossover points at 15 February, 1 April, 14 April, 17 May, 15 July, and 20 September, respectively.

\subsection{Application of the index}

The NRM index is tested for many different monsoon regions in the world. The NRM's onset dates are then compared to the relevant studies in the pass. The comparable ODs among the former and our newer index implies that NRM is reliable. A striking advantage of the NRM index is the capacity of identifying the withdrawal dates. The variation of monsoon duration (OD-WD) will be effected to the monsoon interannual variability. The NRM index is the first global unified index that has such characteristic. Tests also show the NRM is not sensible to the choice of domain. Over a specific region, there is not much different result when we increase/decrease the domain size. An empirical rule has been promoted and it shows the satisfaction to all monsoon regions. 


\subsubsection{OD-WD in Vietnam and the Southeast Asian Sea region}

Here we use the REG2 region as an example for demonstrating the detailed application of the index. The process of detecting OD-WD discussed in this section can be applied to all monsoon regions. Figure 5.5 shows the OD-WD in the region in 2010. The longest positive period of NRM starts on 17 May and ends on 16 August. Using the 5-75 rule, the OD and WD in 2010 occur on 3 May and 22 September, respectively. This process was repeated for each year during 1979-2010. The mean OD in 1979-2010 over the REG2 region in this study is 10 May (standard deviation is 21 days). This is consistent with findings from several studies such as Pham et al. (2010), ZW08, and Wang et al. (2004) who found the mean OD over the Indochina peninsula to be during the first weeks of May.

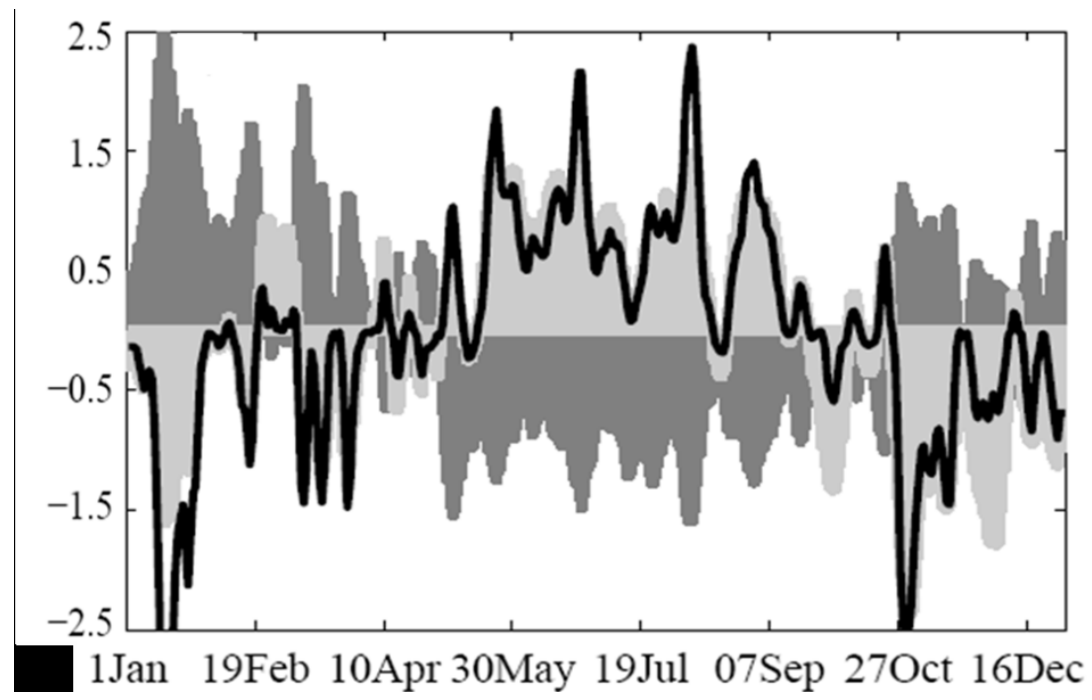

Figure 5.5 NRM index (bold solid black), MSLP (dark gray shaded), and U850 (light gray shaded) in 2010 over the REG2 region $\left(7.5-25^{\circ} \mathrm{N}, 100-120^{\circ} \mathrm{E}\right)$. Y axis: standardized anomaly; $\mathrm{X}$ axis: daily, 5day moving average. 
The mean withdrawal date of the summer monsoon rainfall in Vietnam is on 21 September (standard deviation is 14 days). Hence, the duration of the summer rainy season is estimated at 134 days (standard deviation is 23 days). In this region, the variation in the length of the rainy season (WD-OD) is statistically significantly correlated with that of OD, but not with WD ( $r=-0.80$ and 0.44 , respectively, using $\mathrm{p}=0.01$ level of significance). In addition, there is no statistically significant correlation between OD and WD $(\mathrm{r}=0.18)$. These results imply that early (late) OD is associated with more (less) rain days, and that OD and WD are statistically independent. The detail of OD-WD in each year during 1979-2010 is presented in Table 5.2.

\begin{tabular}{llllllll}
\hline Year & OD & WD & Duration & Year & OD & WD & Duration \\
\hline 2010 & $03 / 05$ & $22 / 09$ & 142 & 1994 & $18 / 04$ & $27 / 09$ & 162 \\
2009 & $18 / 05$ & $24 / 10$ & 159 & 1993 & $21 / 05$ & $18 / 09$ & 120 \\
2008 & $01 / 05$ & $01 / 09$ & 123 & 1992 & $16 / 05$ & $24 / 09$ & 131 \\
2007 & $28 / 07$ & $09 / 10$ & 73 & 1991 & $06 / 06$ & $14 / 10$ & 130 \\
2006 & $16 / 05$ & $09 / 10$ & 146 & 1990 & $10 / 05$ & $22 / 09$ & 135 \\
2005 & $23 / 04$ & $04 / 10$ & 164 & 1989 & $14 / 04$ & $20 / 09$ & 159 \\
2004 & $08 / 05$ & $17 / 09$ & 132 & 1988 & $19 / 05$ & $14 / 09$ & 118 \\
2003 & $02 / 05$ & $28 / 09$ & 149 & 1987 & $30 / 04$ & $15 / 09$ & 138 \\
2002 & $10 / 05$ & $19 / 09$ & 132 & 1986 & $08 / 05$ & $21 / 09$ & 136 \\
2001 & $29 / 04$ & $28 / 09$ & 152 & 1985 & $24 / 05$ & $10 / 09$ & 109 \\
2000 & $14 / 04$ & $17 / 09$ & 156 & 1984 & $17 / 04$ & $10 / 09$ & 146 \\
1999 & $10 / 04$ & $23 / 09$ & 166 & 1983 & $08 / 05$ & $05 / 10$ & 150 \\
1998 & $15 / 05$ & $13 / 08$ & 90 & 1982 & $27 / 05$ & $28 / 09$ & 124 \\
1997 & $01 / 05$ & $18 / 09$ & 140 & 1981 & $08 / 05$ & $06 / 09$ & 121 \\
1996 & $15 / 06$ & $01 / 09$ & 78 & 1980 & $20 / 04$ & $20 / 09$ & 153 \\
1995 & $10 / 05$ & $23 / 09$ & 136 & 1979 & $03 / 05$ & $05 / 09$ & 125 \\
AVE & $10 / 05$ & $21 / 09$ & 134 & STDEV & 21 days & 14 days & 23 days
\end{tabular}

Table 5.2. Onset dates (OD), Withdrawal dates (WD), and Duration between OD and WD (days) of summer monsoonal rainfall in Vietnam during 1979-2010. 32-years average and standard deviation of OD, WD, and Duration are described by AVE and STDEV, respectively. 


\subsubsection{Trend of changes of OD-WD rainfall in Vietnam}

The NRM index allows us to investigate the rainfall variability in more detail compared to the work conducted in the Chapter 4 (Section 4.3.1). In Chapter 4, monthly rainfall is used. Here we use the daily rainfall. Consideration the climatological distribution of rainfall (Chapter 3), 30 November every year is chosen as the last day of the rainy season in Vietnam. Matsumoto (1997) and Wang and LinHo (2002) also used November as the end of rainy season in Vietnam. Rainfall from the withdrawal day to 30 November can be considered to be the non-monsoon or post-monsoon rainfall.

Rainfall variability during monsoon (OD-WD) and post-monsoon (WD-30November) in the country is re-examined. Both the Mann-Kendall and the least squared method are used for the same objective, and the results show that trends are not sensitive to the methodology. Compared to the Chapter 4 results where IS and N3 are the two sub-regions that have shown significant trends (IS increases, N3 decreases), here we have more detailed results. Table 5.3 shows the trends of total rainfall in both monsoon and post-monsoon in each climate subregion. Decrease is the general trend for rainfall in Vietnam in the last 32 years. The nonmonsoon rainfall over the whole country is reduced, particularly those in the northern stations and Central Vietnam (N4 and S1 sub-regions). Although the rainfall in Central during monsoon is increased, it is difficult to identify the mechanism of this increase. This increase can come from monsoon, vortex, or both these two processes. The trends received by the least squared method are shown by Figure 5.6.

\begin{tabular}{lllllllllll}
\hline Observation & N1 & N2 & N3 & N4 & S1 & S2 & S3 & IS & C & AVRI \\
\hline OD-WD & $\downarrow$ & $\downarrow \downarrow \downarrow$ & $\downarrow$ & $\uparrow$ & $\uparrow \uparrow$ & $\downarrow$ & $\downarrow$ & $\uparrow$ & $\uparrow$ & $\downarrow$ \\
WD-30Nov & $\downarrow \downarrow$ & $\downarrow \downarrow \downarrow$ & $\downarrow$ & $\downarrow \downarrow \downarrow$ & $\downarrow$ & $\uparrow$ & $\downarrow$ & $\downarrow$ & $\downarrow$ & $\downarrow$
\end{tabular}

Table 5.3. Trends of changes of rainfall in monsoon and post-monsoon period, discriminated by the NRM monsoon index, in each climate sub-region. Trends are identified by the Mann-Kendall test. The signs $\{\uparrow, \downarrow\}$ denote the increase or decrease trend. One arrow means that trend is not significant. Two, three, and four arrows represent for 90\%, 95\% and 99\% confidence interval for the existence of trend. $\mathrm{N}(1-3), \mathrm{S}(1-3)$, IS, C, and AVRI represent for climate sub-region in northern (N), southern(S), island (IS), Central (C), and Vietnam, respectively. OD, WD, 30Nov denote for the monsoon onset day, monsoon withdrawal day, and 30 November. 

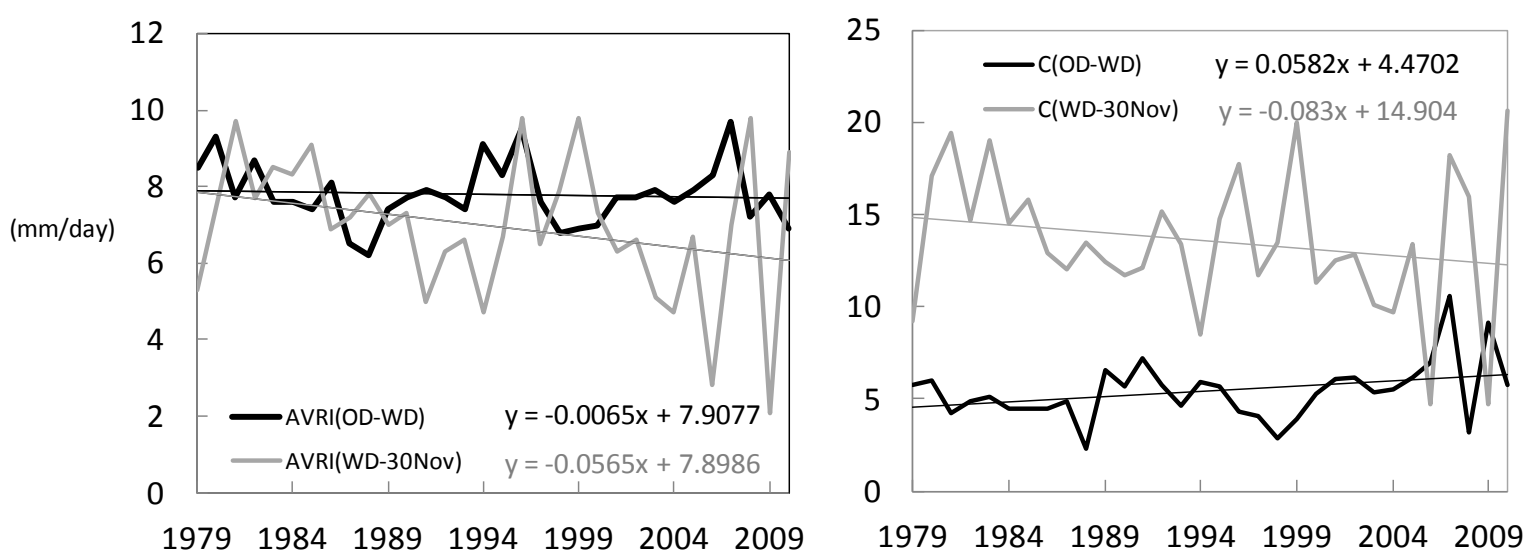

Figure 5.6. Trends of rainfall in Vietnam (left) and Central Vietnam (right) during the monsoon (black) and post-monsoon (gray) period. Trends are detected by the least squared method. AVRI (left) and C (right) denote for the All-Vietnam summer monsoon Rainfall Index (AVRI) and Central Vietnam. OD, WD, 30Nov denote for the monsoon onset day, monsoon withdrawal day, and 30 November. Unit: $\mathrm{mm} /$ day.

From the monsoon index perspective, this discrimination, monsoon and non-monsoon rainfall, allows us to have a better link with the NRM index and, as a result, a greater opportunity of predictability of the monsoon rainfall.

The predictability of the non-monsoon rainfall depends on the mechanism that it may be associated with. Recall that the post-monsoon period is also the time of the rainy season in the Central, and typhoon was seen as the big player contributes to the rainfall in this region (Chapter 3, Section 3.6.1). Therefore the predictability of non-monsoon rainfall is likely depended on the typhoon predictability. The trends revealed here may imply that the typhoon activity, especially during the post-monsoon in 1979-2010 in Vietnam has declined. The variability of rainfall in Central will be discussed in more detailed in the next chapter where the vortex activity and its associated rainfall are investigated.

\subsubsection{OD-WD in other major monsoon regions}

The onset-withdrawal phases in all other monsoon regions (India, Australia, Africa, and the Americas) are investigated for specific regional domains as carried out in previous studies (Figure 5.7). 

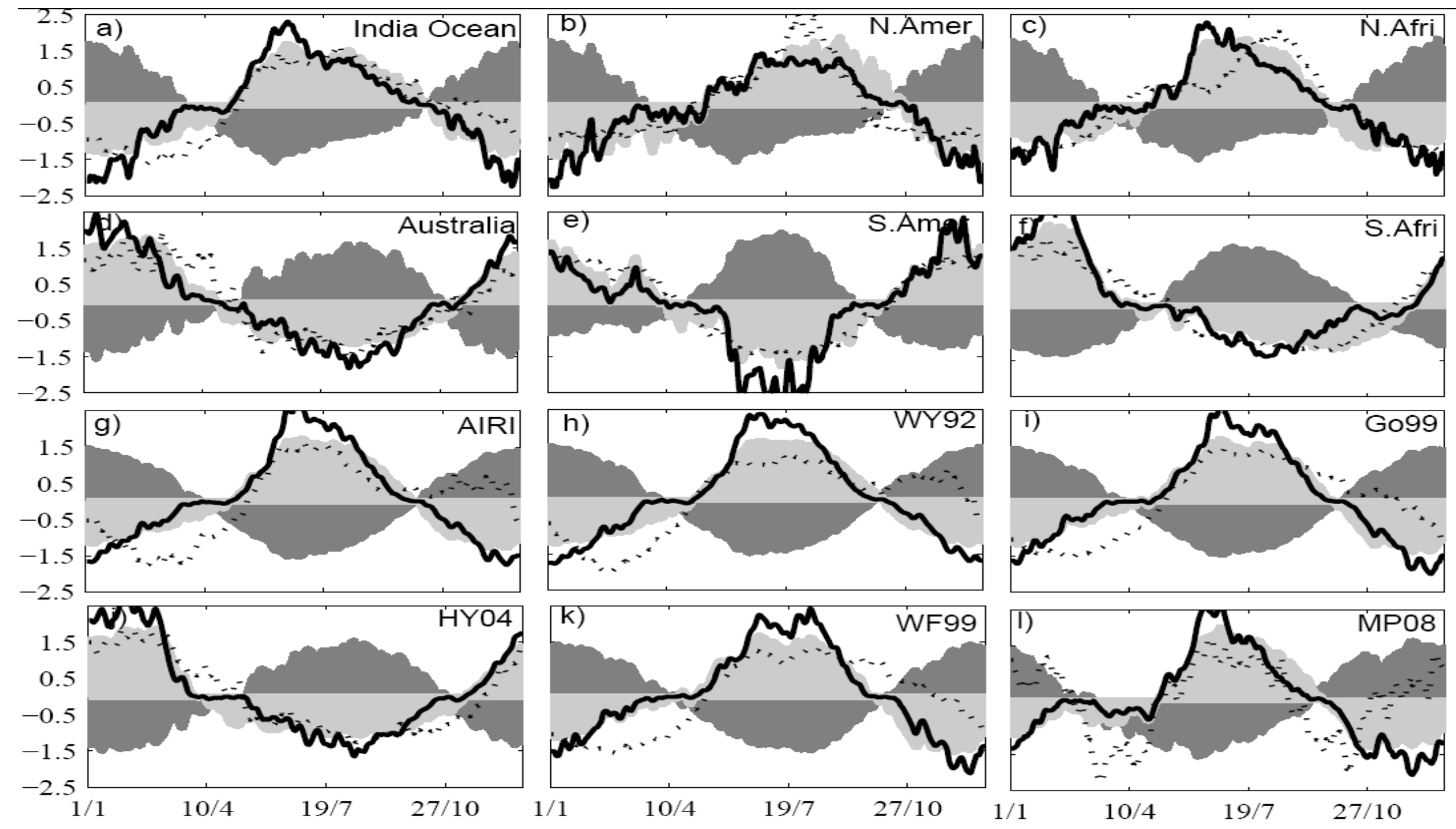

Figure 5.7. Average of monsoon onset-withdrawal phases for 1979-2010 in: a) India Ocean(5-15N, 85-95E), b) North America (30-40N, 110-100W), c) North Africa (5-15N, 35-45E), d) Australia (10S-EQ, 135-145E), e) South America (17.5-7.5S, 52.5-42.5W), f) South Africa (17.5-7.5S, 35-45E), g) All-India summer monsoon Rainfall domain, Including extension regions (AIRI) (5-30N, 65-90E), h) Webster-Yang (1992) index domain (0-20N, 40-110E), i) Goswami et al. (1999) index domain (10-30N, 70-110E), j) Hung and Yanai (2004) index domain (15-2S, 115-150E), k) Wang and Fan (1999) index domain (5-32.5N, 90-140E), and 1) McPhaden et al. (2008) region (30S-30N, 30-120E). MSLP (dark gray shaded), U850 (light gray shaded), rainfall (dotted), Monsoon Index (black). X-axis: daily, 5-day moving average from 01 January, Y-axis: standardized anomalies. 
The average values of OD-Peak-WD and the duration of the rainy season over the Indian Ocean, North America, North Africa, Northern Australia, South America, and South Africa from the current study are in agreement with those revealed by the previous studies for the same regions (Ananthakrishnan et al., 1981; Liebmann et al., 2008; Seleshi and Zanke, 2004; Pope et al., 2009; Garcia et al., 2011; and Cook et al., 2012, respectively). For example, Garcia et al. (2011) identified the average OD over the central Brazil at pentad \#57 (08-12 October). Our finding for the same region is 13 October (Figure 5.7e). Moreover, while the above studies have paid little attention to WD, our index has the advantage of also determining monsoon withdrawal dates. The NRM index has also been applied over other domains specified by previous studies, such as India (Mooley and Parthasarathy, 1984), South Asia (Webster and Yang, 1992), extended India region (Goswami et al., 1999), Northern Australia (Hung and Yanai, 2004), Southeast Asia (Wang and Fan, 1999), and Africa-AsiaAustralia (McPhaden et al., 2008). The NRM index appears to adequately quantify the monsoon variability over those domains, as illustrated in Figure 5.7g-1.

In the Northern Hemisphere most tropical regions witness prevailing westerlies during the summer season. However there are some regions where the prevailing wind is easterly in summer, such as the North American monsoon region (Figure 5.7b). For those regions, the NRM index is calculated using minus U850. The mean OD (WD) in the core North American monsoon region is found to be 26 June (16 September), and the peaks of rainy season occur from July to September. These results are quite consistent with Liebmann et al. (2008). 


\section{CHAPTER 6}

\section{TROPICAL VORTEX AND ITS ASSOCIATED RAINFALL}

There are two possible outcomes: if the result confirms the hypothesis, then you've made a measurement. If the result is contrary to the hypothesis, then you've made a discovery.

\section{Enrico Fermi}

Rain-producing processes in tropical regions usually overlap; consequently it is difficult to get a clear picture of how much each individual process contributes to the total rainfall. With the establishment of the NRM monsoon index (Chapter 5), the rainy season in Vietnam may now be considered as two main periods: monsoon (between onset and withdrawal dates) and post-monsoon (after withdrawal). This discrimination provides a better opportunity to understand the physics of rainfall mechanisms, particularly the vortex-induced rainfall in the post-summer period.

Vortices and typhoons from the western North Pacific significantly contribute to precipitation in Central Vietnam. This vortex-induced rainfall is considered to be the nonmonsoon rainfall. This chapter studies the climatological behavior of tropical vortices over the Southeast Asian Sea - Maritime Continent region. The results show that vortices in this region exist almost year-round, migrating from the coast of Vietnam - Southeast Asian Sea in summer to the Borneo Island region in winter. Clear evidence is found for the presence of a semi-permanent vortex near the south coast of Vietnam, the northern central part of the region. The seasonal march of vortex shows that the Borneo vortex, a well-known winter vortex in the region, is just the winter component of an annual cycle of vortex activity. 


\subsection{Background}

Rainy season in most climate sub-regions of Vietnam starts in May and ends in September. In these sub-regions rainfall can be linked to south-west monsoon activity. In Central coast, however, rainfall peaks in September-October-November with the linkage to north-east monsoons.

Studies of climate in Vietnam (e.g.: Bruzon and Carton, 1930; Yen et al., 2011) showed that the maximum monthly rainfall in this country varies from North to South in accordance with the seasonal cycle. They also observed the Central rainfall consists of northeasterly monsoon-induced rainfall and tropical cyclone-induced rainfall. Tropical cyclone activity over the Vietnam region (1979-2010) shown in Chapter 3 confirms that typhoons migrate from North to South along with seasonal change. In June-July, typhoons mostly land in the North Central; they are concentrated in the Central region in August-September-October; and then move southward in November. This evidence suggests that the rainfall in Central Vietnam has linkages to tropical cyclones.

In general, a strong tropical vortex can significantly contribute the quantity of rainfall for the whole rainy season at specific locations in a matter of a few days. For example, on 26/09/1980, 24h rainfall recorded in Dong-Ha station $\left(16.9^{\circ} \mathrm{N}, 107.1^{\circ} \mathrm{E}\right)$, a station in Central Vietnam, was $381 \mathrm{~mm}$, which accounted for about $26 \%$ of the total rainfall in October or $11 \%$ of the total annual rainfall at that station (Figure 6.1). Note that this heavy rainfall episode was not caused by a typhoon or strong tropical storm. It was just a low pressure system (or tropical vortex), which can be associated with such large amounts of rainfall. A strong vortex system can produce as much daily rainfall as 582mm (Fabian typhoon, 10/10/1981, Hue station, another station in the Central Vietnam) or even the record-breaking $977 \mathrm{~mm}$ on $03 / 11 / 1999$ (same station). In addition, the heavy rainfall on $26 / 09 / 1980$ is considered to be the post-summer monsoon rainfall since the OD-WD of Vietnam in 1980 is from 20 April to 20 September (Chapter 5). The terms post-monsoon, post-summer, or non-monsoon rainfall have the same meaning, describing rainfall in the period from the WD onwards to the end of the rainy season, and is used interchangeably in this study. 


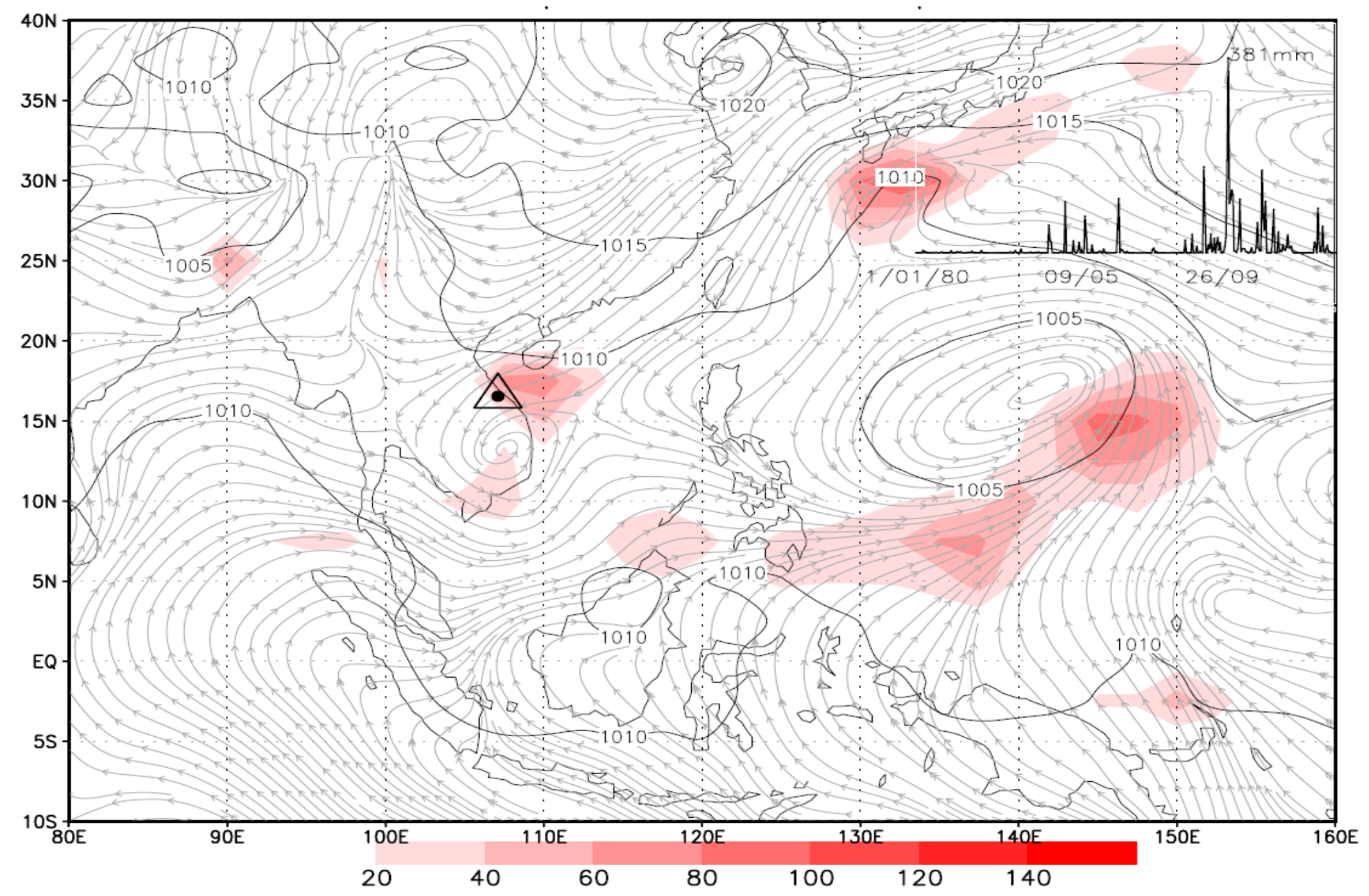

Figure 6.1. JRA-25 reanalysis streamlines at $925 \mathrm{hPa}$ at 12UTC 26 September 1980. Black contours denote the mean sea level pressure, pink shaded areas represent the $24 \mathrm{hr}$ rainfall $(\mathrm{mm})$. The vortex produced $381 \mathrm{~mm} / 24 \mathrm{~h}$ at Dong-Ha station (WMO code \# 48849, triangle dot). 
When the tropical vortex season ceases in Vietnam, however, the maximum climatological rainfall centres keep moving southwards. Studies, such as Sadler and Harris (1970), Meehl (1987), and Chang et al. (2006), found the migration of a rainfall centre from the Indochina peninsula in October to the eastern coast of the Malay peninsula and the west of Borneo Island $\left(2.5^{\circ} \mathrm{N}, 110^{\circ} \mathrm{E}\right)$ in November-December, which then migrated southward to the Java Sea and other Indonesian islands in January-February. Borneo Island lies on the path of this migration. The mechanism of this shift has been considered to be monsoon trough movement (Sadler and Harris, 1970), or the interaction between the penetration of northeasterly monsoon winds and the orography (Chang et al., 2006). Chen et al. (2012b) estimated that the Borneo vortex causes about $60 \%$ of total rainfall in north of Borneo Island. The Borneo vortex was considered to be a typical winter tropical vortex in the region.

In December 1978, the Winter Monsoon Experiment (WMONEX) was established with the objectives to study both the global and regional aspects of the winter monsoon circulation over East Asia, the Maritime Continent, and Australia (Johnson and Chang, 2006). WMONEX was the first and biggest international effort to create a consistent data set for that specific research purpose. The WMONEX programme made a significant contribution to the understanding of rain-producer cloud systems, diurnal convection, easterly waves, cold surges, and the Borneo vortex. The mechanism of winter rainfall in the Maritime Continent, therefore, was partly explained by the presence of the Borneo vortices, formed by the equatorial easterly wave disturbances (Riehl et al., 1948), the wind shear between two opposing flows (Sadler, 1967), or the interaction between cold surges from the North and the monsoon trough (Cheang, 1977). Some of the earlier studies of the Borneo vortex are listed in Table 6.1. 


\begin{tabular}{|c|c|c|c|}
\hline Authors (Years) & Period & Domain & Key words \\
\hline Ramage (1968) & $12 /\{1963,1964\}$ & $20 \mathrm{~S}-30 \mathrm{~N}, 90-160 \mathrm{E}$ & Latent heat released. \\
\hline Cheang (1977) & $12 / 1973$ & $20 \mathrm{~S}-30 \mathrm{~N}, 90-140 \mathrm{E}$ & Borneo vortex genesis. \\
\hline Chang et al. (1979) & $12 / 1974$ & $5 \mathrm{~S}-25 \mathrm{~N}, 100-125 \mathrm{E}$ & Cold surges and vortices. \\
\hline Houze et al. (1981) & $12 / 1978$ & Borneo Island & $\begin{array}{l}\text { Structure and time variation of } \\
\text { cloud and precipitation. }\end{array}$ \\
\hline $\begin{array}{ll}\text { Churchill } & \text { and } \\
\text { Houze (1984) }\end{array}$ & $12 / 1978$ & EQ-20N,100-120E & $\begin{array}{l}\text { Structure and development of } \\
\text { cloud clusters. }\end{array}$ \\
\hline $\begin{array}{l}\text { Johnson and } \\
\text { Houze(1987) }\end{array}$ & 12/1978-03/1979 & $20 \mathrm{~S}-20 \mathrm{~N}, 100-120 \mathrm{E}$ & Convective clouds. \\
\hline Chang et al. (2003) & $12 / 2001$ & $\begin{array}{l}10 \mathrm{~S}-12.5 \mathrm{~N}, 100- \\
120 \mathrm{E}\end{array}$ & Vamei typhoon. \\
\hline Chang et al. (2005) & $\begin{array}{l}12-02 /\{1980- \\
2001\}\end{array}$ & $10 \mathrm{~S}-15 \mathrm{~N}, 90-120 \mathrm{E}$ & $\begin{array}{l}\text { Borneo vortex, Madden-Julian } \\
\text { Oscillation, and cold surges. }\end{array}$ \\
\hline $\begin{array}{l}\text { Tangang et al. } \\
(2008)\end{array}$ & $12 / 2006-01 / 2007$ & $10 \mathrm{~S}-10 \mathrm{~N}, 90-130 \mathrm{E}$ & $\begin{array}{l}\text { Borneo vortex, Madden-Julian } \\
\text { Oscillation, and Indian Ocean } \\
\text { Dipole. }\end{array}$ \\
\hline
\end{tabular}

Table 6.1. Tempo-spatial features on structure and evolution of winter tropical disturbances over the southern Southeast Asian Sea in or close to the framework of the Winter Monsoon Experiment.

Tropical cyclone activity over the Southeast Asian Sea and western North Pacific region usually ceases in November-December. However the southward migration of an aforementioned climatological rainfall centre in the region persists in the following winter months. Apart from the involvement of the north-to-south movement of typhoons, the migration of a regional rainfall centre suggests an additional mechanism associated with rainfall.

Table 6.1 above shows that only a few studies have considered the wider Southeast Asian Sea - Maritime Continent domain, the majority focusing on a limited region and specific time. In this chapter, we study the tropical vortex activity with consideration of the spatialtemporal extension of the Borneo vortex. In other words, we broaden the domain of study from the Borneo region to cover the full Southeast Asian Sea - Maritime Continent region, and we are interested in vortex activity throughout the year. 


\subsection{Method}

The Nguyen and Walsh (2001) vortex threshold criteria (wind speed at 10-m level greater

than $5 \mathrm{~m} / \mathrm{s}$ and relative vorticity at $850 \mathrm{hPa}$ greater than $10^{-5} \mathrm{~s}^{-1}$ ) are used to detect tropical vortices. Any vortex centre less than $500 \mathrm{~km}$ from coast is considered to be one that can produce rainfall over Vietnam. This $500 \mathrm{~km}$-radius threshold is used widely in both research (Marks et al., 2002; Hattori et al., 2010; Dare, 2013) and operational settings (NOAA, Hongkong Observatory, NCHMF Vietnam) for the same objective. IBTrACS-WMO tropical cyclones tracks are also used to recognise tropical cyclones from other vortices.

An algorithm is developed for the region. Every day from the monsoon onset dates (Nguyen et al., 2014b) to 30 November (over all years) is checked, and if thresholds are exceeded, 1) the observed rainfall is extracted and classified as vortex-induced rainfall (VR), either monsoon or post-monsoon, and 2) VR days are counted by the number of rain days at stations. VR is then analysed in terms of the frequency of occurrence of VR days and the spatial-temporal distribution of rainfall (monsoon VR and post-monsoon VR). For the purpose of visualization in Figure 6.5, the number of vortex-induced rainfall days at stations are interpolated to a regular $1 \times 1$ degree grid.

\subsection{Initial results}

Chang et al. (2006) described the propagation of rainfall in the region; here we re-examine this phenomenon with newer reanalysis datasets and using a different approach. Stations in eight of the ten countries in the Association of Southeast Asian Nations (ASEAN) are shown in Figure 6.2. Both continental and ocean rainfall patterns show consistency with Chang et al. (2006) results. This shows that there is a migration of rainfall over this region, from the North Southeast Asian Sea (SEAS) in September-October to the South Maritime Continent in January-February. 

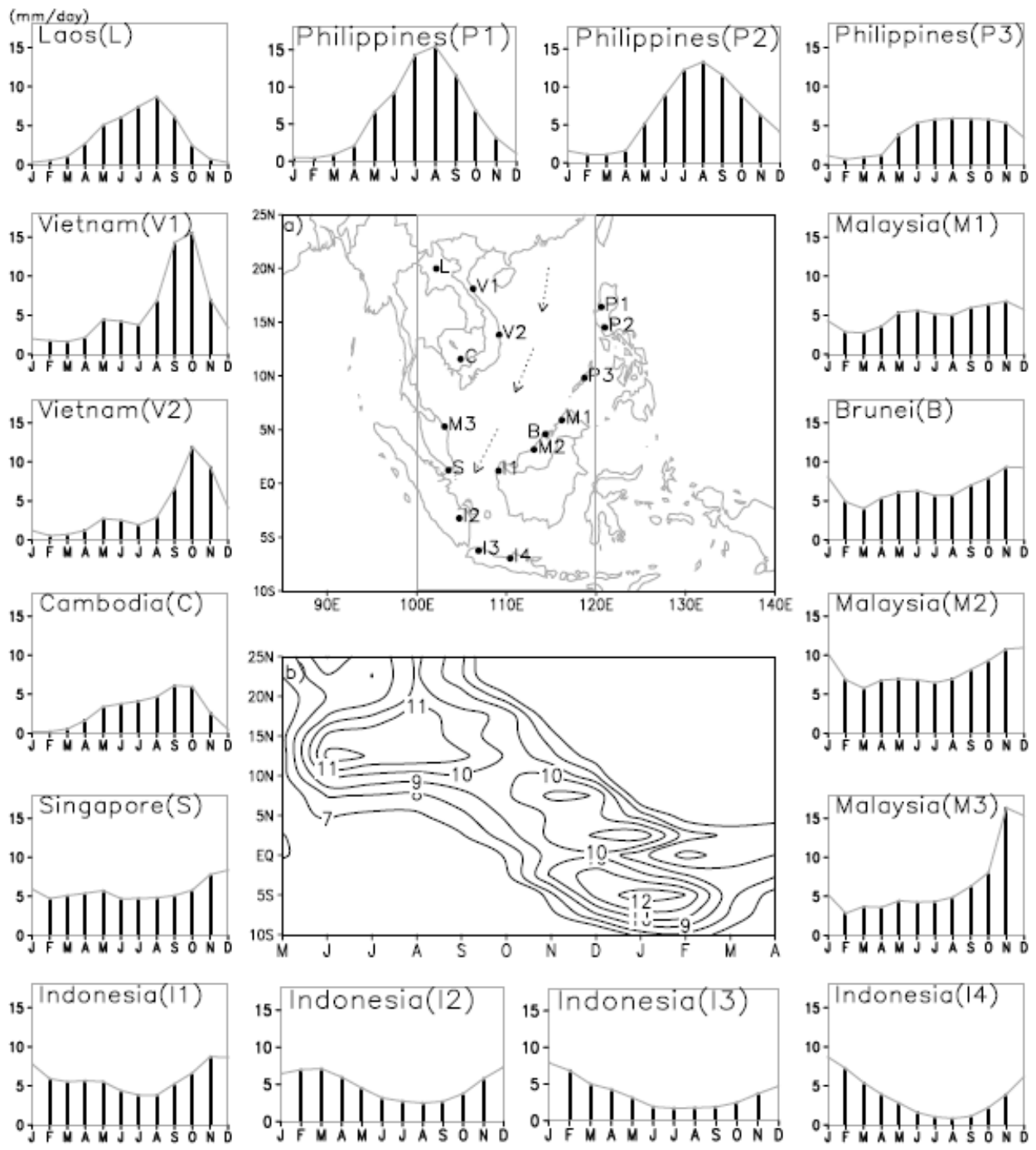

Figure 6.2. a) Monthly rainfall of Asean stations from APHRODITE. Used stations: Laos(L(19.98N,102.18E)), Vietnam (V1(18.1N,106.3E), V2(13.8N,109.2E)), Cambodia (C(11.56N,104.9E)), Singapore (S(1.21N,103.54E)), Philippines

(P1(16.4N,120.6E), P2(14.5N,121E), P3(9.8N,118.7E)), Malaysia (M1(5.88N,116.19E), M2(3.15N,113.09E), M3(5.28N,103.1E)), Brunei (B(4.58N,114.36E)), and Indonesia(I1(1.16N,109.14E), I2(3.25S,104.75E), I3(6.27S,106.9E), I4(6.97S,110.43N)). Y-axis (mm/day). Core domain of study: (10S-25N, 100-120E). b) Hovmoller diagram of monthly CMAP rainfall over ocean, data being averaged over 100-120E. Only contours greater than $7 \mathrm{~mm} /$ day are displayed.

Temporal translocation of rainfall over the region is also illustrated by dotted arrows in a). Unit: $\mathrm{mm} /$ day. 
Counts of the existence of vortex centers, calculated by adding up all monthly occurrences of vortices at each grid point in the last 32 years (1979-2010) are presented in Figure 6.3. Around Borneo Island there is a high concentration of vortex occurrences, as found in previous studies. The interesting finding here is that vortices can appear over the entire Southeast Asian Sea - Maritime Continent region, not only the Borneo - Singapore region. A closer look at monthly activity of these vortices is made. Figure 6.4 uses different reanalysis datasets to illustrate the vortex occurrences and locations. In all reanalysis datasets, the summer-to-winter movement of vortex occurrence in the NCEP-DOE reanalysis is shown the most agreement with the rainfall distribution figured out by Sadler and Harris (1970), Meehl (1987), or Chang et al. (2006). Statistics on vortex, hereafter, will be conducted with NCEPDOE reanalysis.

The average frequency of vortex occurrences (in terms of the percentage of months where a vortex is present) varies by season, decreasing from about $80 \%$ in December-JanuaryFebruary, to $75 \%$ in September-October-November, $60 \%$ in June-July-August, and down to $50 \%$ in March-April-May. In summer months (June-July-August), vortex locations are farthest north, about $21^{\circ} \mathrm{N}$, extending zonally from the Gulf of Tokin to the central North SEAS. Three months later, their mean position is near the central coast of Vietnam-SEAS; during this time a vortex can form as far south as Borneo Island. In winter the vortex mean position is around Borneo.

Vortices, as defined so far, could include TCs as well as less intense vortices over the SEAS. In fact, criteria to detect vortices do not have any indication of the mechanism of formation and movement. In other words, vortices in Figure 6.3 and 6.4 include not only those forming and functioning inside the Southeast Asian Sea - Maritime Continent region, but all typhoons, TCs coming to the region from the north western Pacific. To confirm the existence of a permanent vortex, we extract all IBTrACS's TCs from daily time-series in reanalysis data sets and then reassess the remaining vortices. The result of this approach is introduced in the next section. 


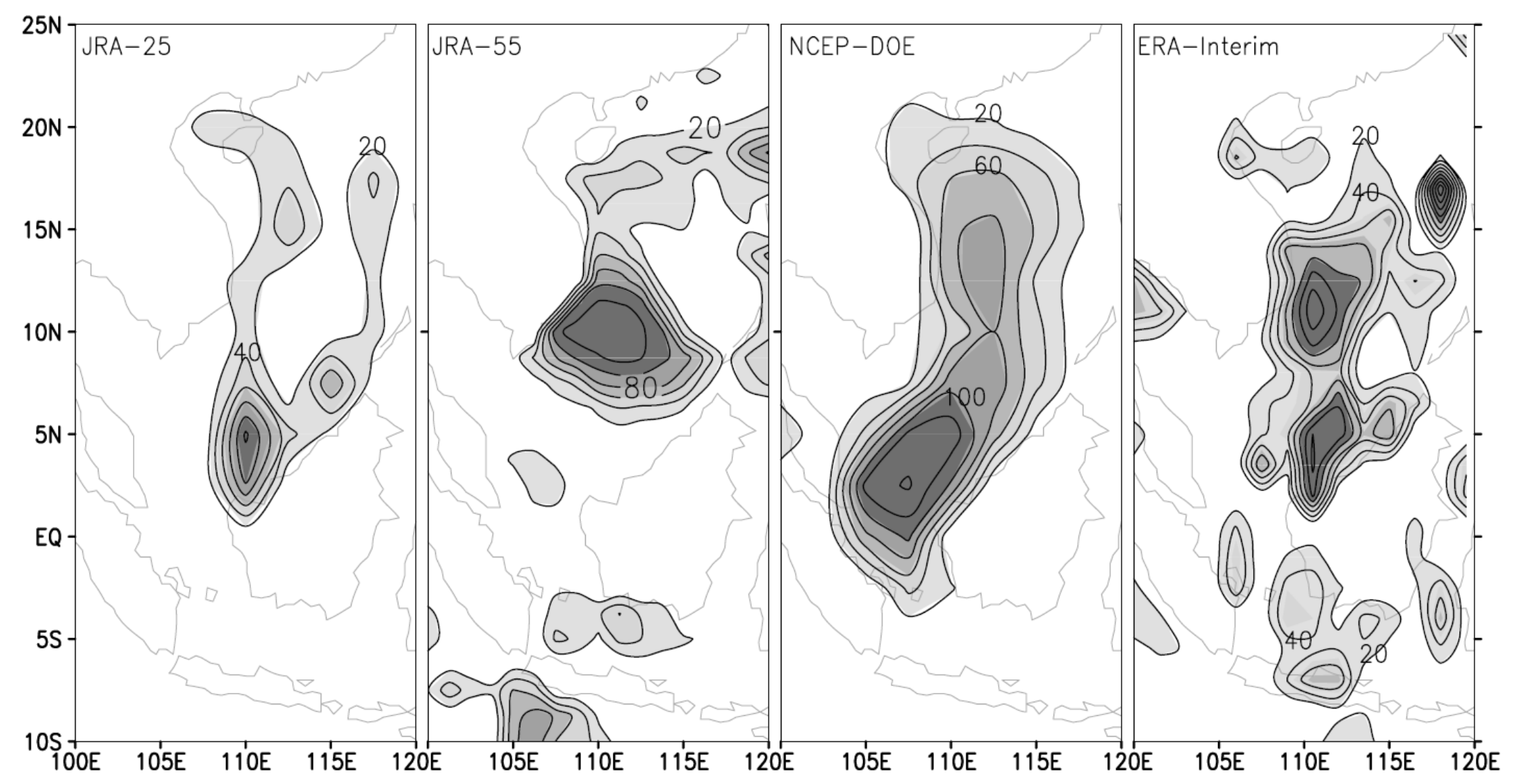

Figure 6.3. Number of appearance of monthly vortices in ocean grid points over the Southeast Asian Sea - Maritime Continent region during 1979-2010. From left to right: JRA-25(2.5x2.5degree), JRA-55(1.25x1.25degree), NCEP-DOE (2.5x2.5degree), and ERA-Interim (1.5x1.5 degree) reanalysis dataset, respectively. Contour interval: 20 . 

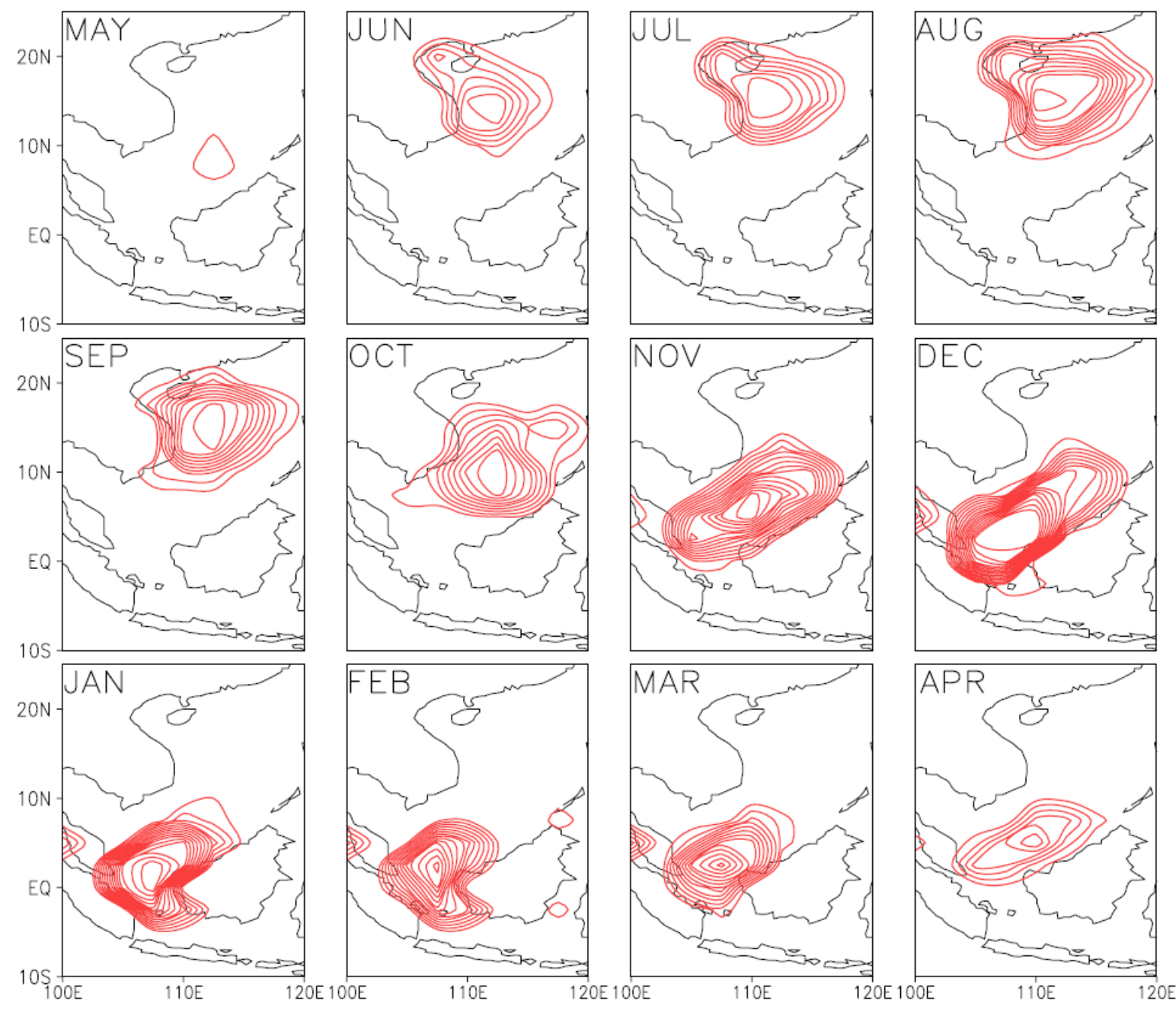

Figure 6.4. a) Monthly occurrence of vortices over the sea in the Southeast Asian Sea - Maritime Continent region in NCEP-DOE reanalysis (1979-2010). Contour lines indicate the frequency of occurrence of vortices in each month. Contour interval: 2. 

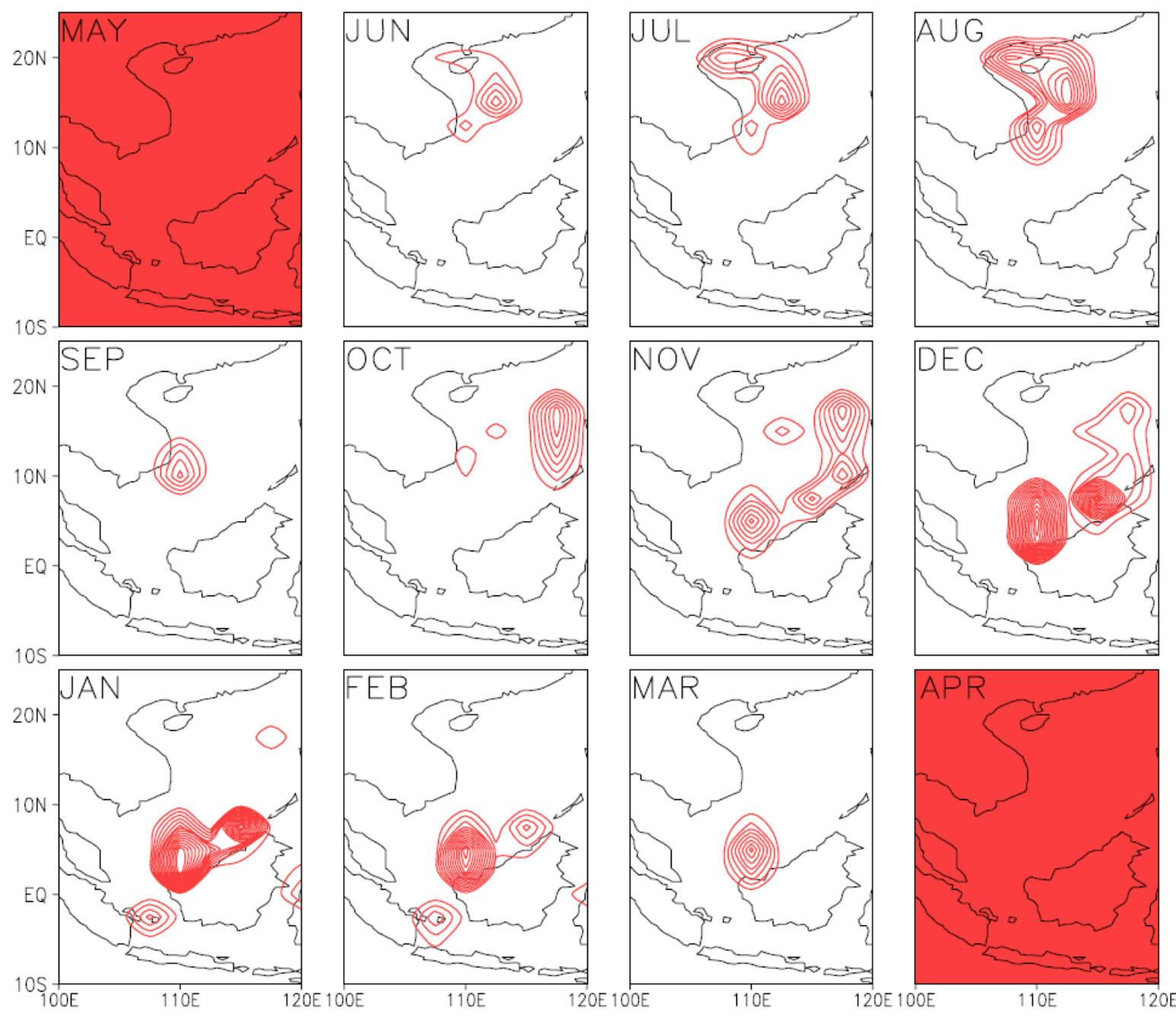

Figure 6.4.b) Same as a) except JRA-25. Red panels April and May represent nil vortices. 

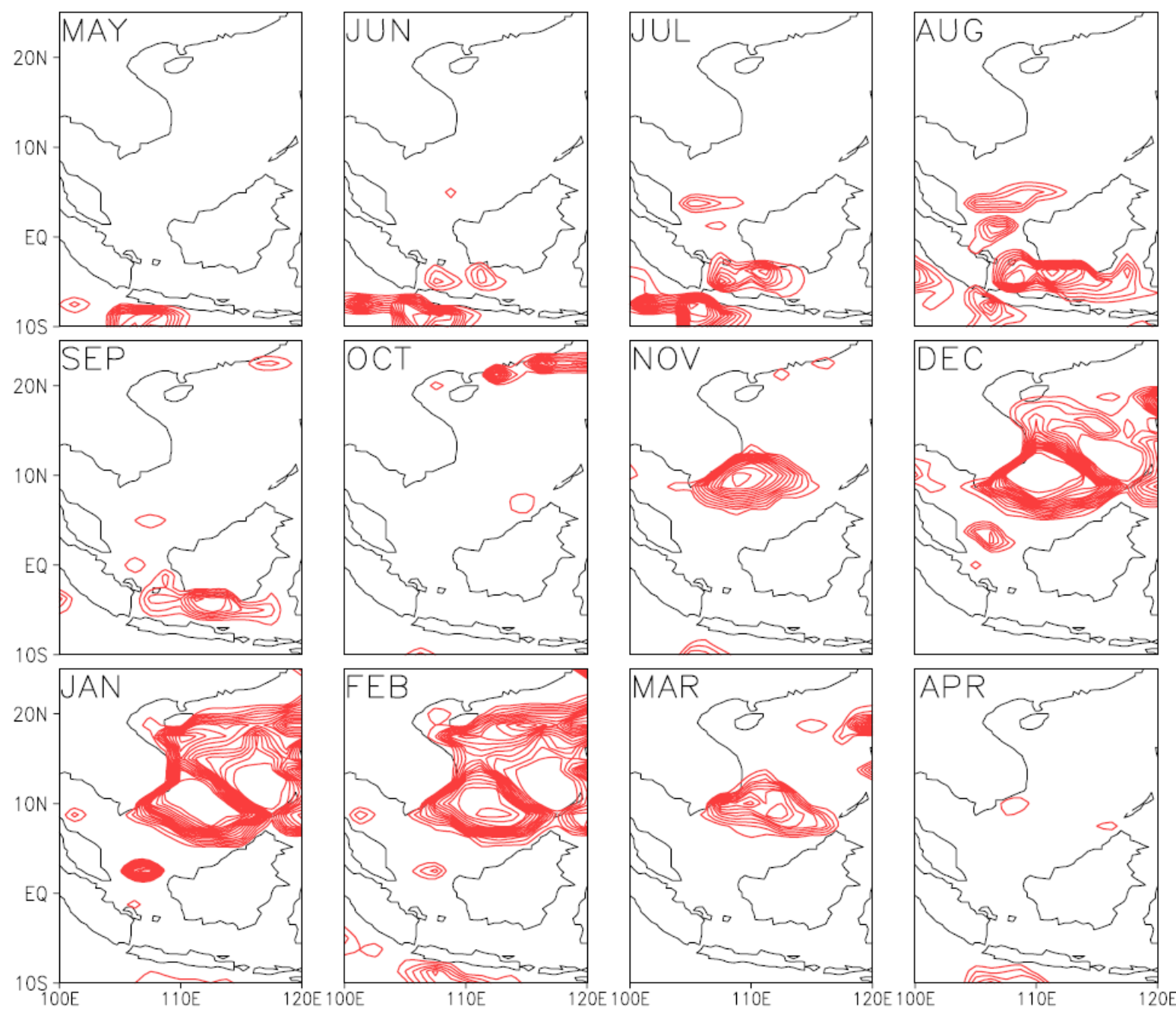

Figure 6.4.c) Same as a) except JRA-55 

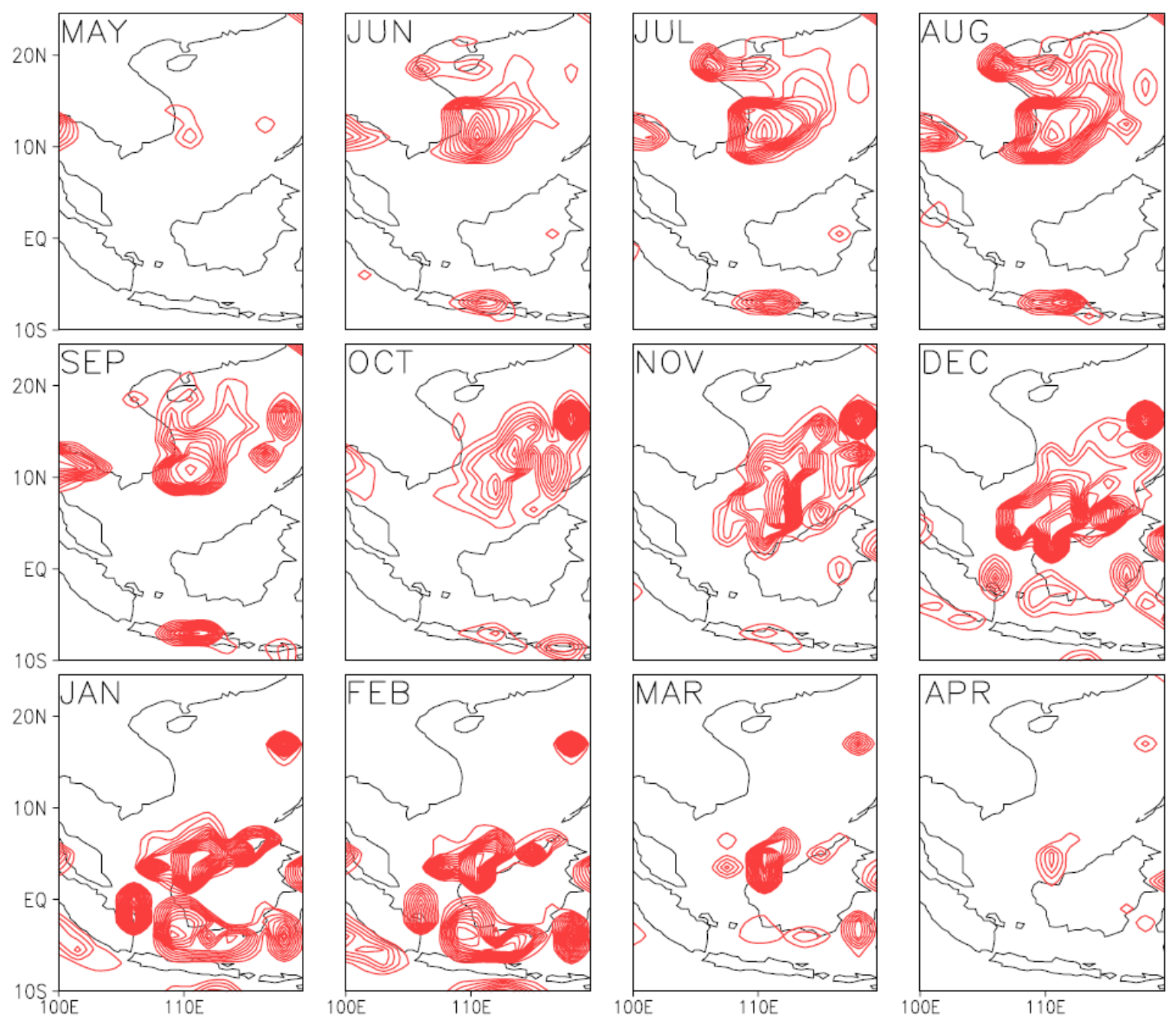

Figure 6.4.d) Same as a) except ERA-Interim 


\subsection{Southeast Asian Sea - Maritime Continent Vortex}

Vortex-induced rain (VR) days at stations are shown in Figure 6.5. In four different reanalysis datasets, the highest and most consistent frequency of rain days is found around the southern coast of Central Vietnam. The maximum number of VR days varies from $50 \%$ in JRA-25 (equivalent to 5840 days of the total 11680 days in 1979-2010) to $80 \%$ in JRA-55 (Figure 6.5 a). There are two points that we can derive from these numbers.

First, we examined if dataset resolution affected our results. A sensitivity test on resolution was made. The JRA-55 and ERA-Interim reanalyses were degraded to $2.5^{\circ} \times 2.5^{\circ}$ latitude/longitude resolution and the VR days were re-calculated. These new VR days patterns in JRA-55 and ERA-Interim are almost the same as NCEP-DOE and JRA-25: the maximum number of VR days is on the southern coast, and is about 50-55\% of total days in 1979-2010 (Appendix Figure AF6). It appears that the presence of a permanent vortex is not sensitive to the interpolation procedure.

Second, the very high number of VR days on the southern coast can be interpreted as follows. The maximum frequency of 50\% (in JRA-25, for instance) means one of every two days (on average) is affected by vortex activity in that region. Look at the IBTrACS-WMO typhoon track for 1979-2010, there are 334 typhoons/tropical cyclones/lows observed over the domain (3-25N, 100-120E) (see Appendix Figure AF3). On average each typhoon over this domain lasts 3-5 days (equivalent to the number of days of rain). Therefore the total WMO typhoon-induced rainfall (TR) days over the region is about 1000-2300 (days) during 19792010 , equivalent to $10-20 \%$ of days in total. Roughly, if we extract TR days from Figure $6.5 \mathrm{a}$, a significant remainder of vortex days should be left. To demonstrate this thinking, all TR days are removed in individual reanalysis data sets, the remaining VR days are shown in Figure $6.5 b$. 

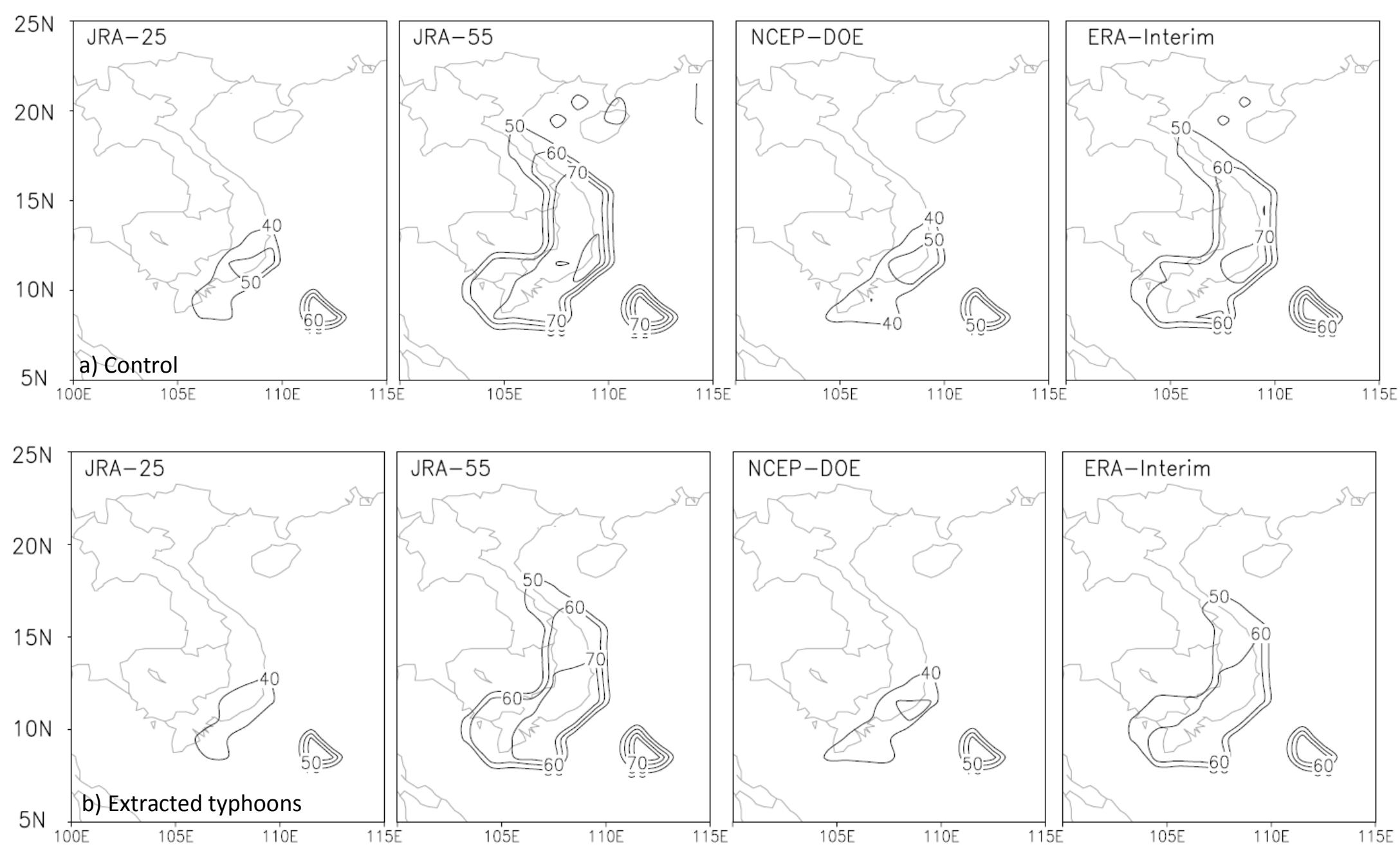

Figure 6.5. a) Vortex-induced rain days at stations (VS), as a percentage of the total (11680) number of days in the 32 year record (1979-2010). b) same as a) except all WMO typhoon-induced rainfall days were removed. Only VS greater than 39\% is displayed. 
The rainfall associated with VR days (Figure 6.5b) demonstrates the presence of a permanent vortex on the South Coast of Vietnam - Philippines. This summer component and the winter component (Borneo vortex) create an annual cycle of a semi-permanent vortex over the Southeast Asian Sea - Maritime Continent (SEAMCV).

\subsection{Vortex activity and post-monsoon rainfall}

The contribution of vortex rainfall to the total precipitation budget in Vietnam is investigated. Daily mean rainfall before and after extracting VR are displayed in Figure 6.6 .

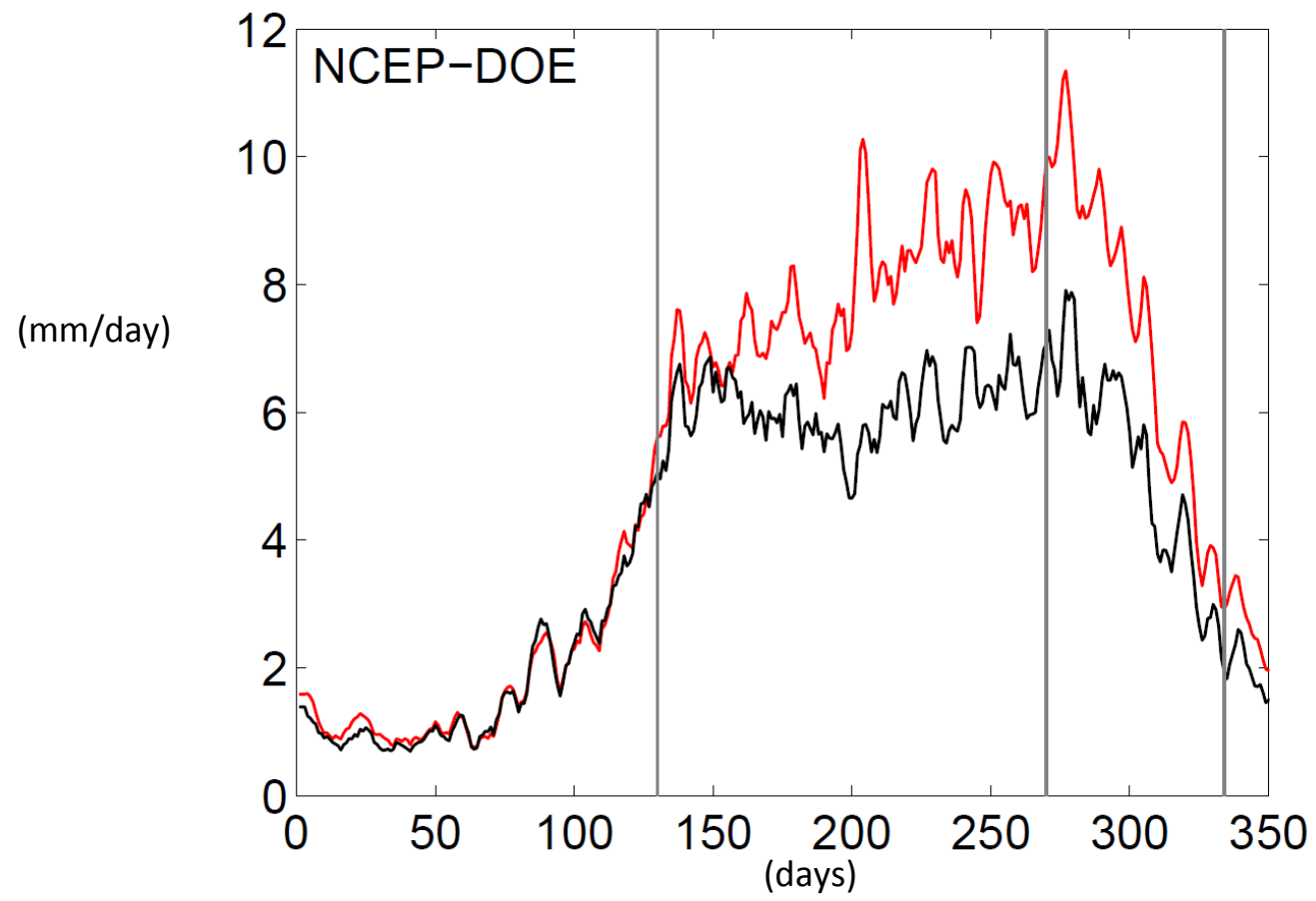

Figure 6.6 Mean temporal distribution of rainfall before (red) and after (black) extracting vortex rainfall in Vietnam (1979-2010). Red and black lines represent observation and vortex-induced rainfall by NCEP-DOE reanalysis dataset, respectively. Vertical lines from left to right denote mean onset (day \#130), withdrawal (day \#264), and 30 November (day \#334). Y-axis: mm/day, X-axis: 365 days. 
VR includes all typhoons and vortices, however the analysis from Section 6.4 shows that TR accounts for only about $10 \%$ in the total VR, therefore VR is mainly consisted of vortex-induced rainfall.

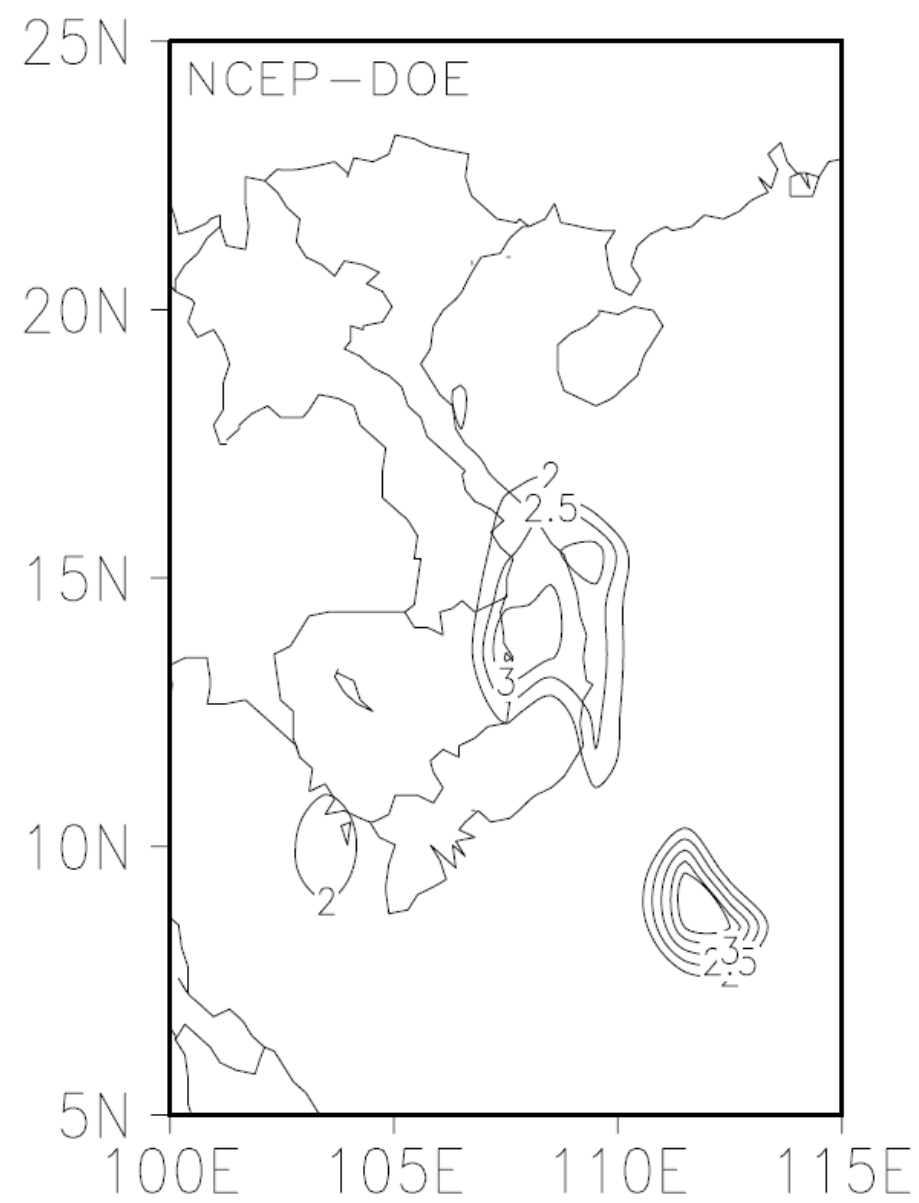

Figure 6.7. Mean spatial distribution of the difference between before and after extracting vortexinduced rainfall ( $\mathrm{mm} /$ day). Only contours greater than $2 \mathrm{~mm} /$ day are displayed.

VR plays a significant role in the whole rainy season, both monsoon as well as postmonsoon period. This feature is consistent in all reanalysis datasets (Appendix Figure AF7). In the last part of this study we will examine the variation of VR with a particular interest in the post-summer period. 
Three key clues supporting the conclusions that tropical vortices are the leading mechanism to producing rainfall in Central Vietnam are listed below:

1) Rainfall in coastal Central peaks in September-October-November.

2) Post-summer rainfall is mostly caused by tropical vortex activity.

3) A vortex exists in coastal Central, and is well observed in September-OctoberNovember.

The first factor is presented in Chapter 1 and Section 3.4; the post-monsoon definition (the second factor) is described in Section 6.2; and the presence of the vortex is introduced in Section 6.3 (Figure 6.3, Figure 6.4), and is complimented by the spatial distribution of VR (Figure 6.6, this section). The role of tropical vortex activity in the rainfall of Central Vietnam will be diagnosed quantitatively in more detail in the next section.

\subsection{Quantitative analysis}

Table 6.2 shows the contribution of vortex-induced rainfall in each period for each climate sub-region in Vietnam. Recall that VR during OD-WD may include monsoon rainfall while VR in post-summer indicates vortex activity.

Overall, VR increases from North to South corresponding with the summer-to-autumn transition of rainfall. This feature is consistent with vortex activity revealed in Section 6.3 and 6.4 . 


\begin{tabular}{lllll}
\hline Resolution & Control & \multicolumn{3}{l}{ Sensitivity test $\left(2.5^{\circ} \times 2.5^{\circ}\right)$} \\
& & & \\
\hline & OD-WD & Post-Summer & OD-WD & Post-Summer \\
\hline N1 & $6-56 \%$ & $9-42 \%$ & $6-40 \%$ & $9-23 \%$ \\
N2 & $9-68 \%$ & $10-50 \%$ & $9-49 \%$ & $10-31 \%$ \\
N3 & $14-68 \%$ & $14-61 \%$ & $14-53 \%$ & $14-42 \%$ \\
N4 & $30-74 \%$ & $29-84 \%$ & $30-65 \%$ & $29-57 \%$ \\
S1 & $72-92 \%$ & $69-94 \%$ & $72-85 \%$ & $67-87 \%$ \\
S2 & $70-88 \%$ & $63-85 \%$ & $70-80 \%$ & $63-79 \%$ \\
S3 & $44-84 \%$ & $43-78 \%$ & $44-65 \%$ & $43-66 \%$ \\
IS & $80-93 \%$ & $78-93 \%$ & $76-89 \%$ & $78-91 \%$ \\
Vietnam & $29-73 \%$ & $41-80 \%$ & $29-59 \%$ & $41-59 \%$
\end{tabular}

Table 6.2. Contribution of vortex rainfall to the total rainfall (\%) during specified periods.

Ranges across datasets represent their minimum and maximum values. The original (control) dataset resolutions are 2.5x2.5 degrees for JRA-25 and NCEP-DOE; 1.25x1.25 degrees for JRA55, and 1.5x1.5 degrees for ERA-Interim. N1-N4, S1-S3, IS stands for North-to-South climate subregions in Vietnam. OD-WD derived from Nguyen et al. (2014b). On average OD-WD covers 125 days, and Post-Summer length is 70 days.

The rainy season in N1-N2-N3 sub-regions has only one peak in middle summer months and ceases in September-October-November (Chapter 3). Maximum vortex precipitation in these regions should be produced during the OD-WD rather than postsummer time. All datasets capture this feature well.

N1-N2 stations are located far inland from the coast and the rainfall regime here is not greatly affected by tropical vortex systems. The biggest difference between datasets, up to $60 \%$, is witnessed in these sub-regions.

Vortices make a very large contribution to the total amount of rainfall in the Central (N4-S1) sub-regions. VR in post-summer is a little higher than in summer, but in general they both contribute from $30 \%$ to $90 \%$ of total rainfall, depending on the reanalysis data set, in both summer and post-summer periods. 
One striking feature is seen in S1, S2 and IS sub-region where consistently very high VR is seen in all datasets. VR accounts for $70-90 \%$ of total rainfall and the range between datasets is about $10-20 \%$. This result suggests that vortex occurrence has its highest frequency in these regions ( $\mathrm{N} 4, \mathrm{~S} 1, \mathrm{~S} 2$, and IS); in other words these vortex centres are well inside the $500 \mathrm{~km}$ radius from stations. This also fits with our previous findings (e.g. Section 6.4).

It is understandable why the OD-WD rainfall is higher than post-summer rainfall in sub-region S3. Over the whole Indochina peninsula the earliest southwesterly is observed in S3. This monsoon wind prevails there from May until autumn; and rainfall is associated with the southwesterly activity (detailed in Chapter 3 ). When the summer monsoon wind ceases, rainfall starts to reduce.

\subsection{Trends of vortex-induced rainfall}

Chapter 4 explores the seasonal changes in rainfall in Vietnam and its sub-regions. Chapter 5 studies, in part, rainfall variability on a OD-WD basis. One of the important findings in Chapter 5 is that post-monsoon rainfall in Central Vietnam has decreased in the last 32 years. We know from Section 6.5 that vortex activity is the main mechanism for producing rainfall in Central. Here we investigate vortex-induced rainfall with a focus on Central rainfall in the post-monsoon season. The trend of change in VR in Central is investigated by both the Mann-Kendall test (Table 6.3) and the least squared method (Figure 6.8). 


\begin{tabular}{|c|c|c|c|c|c|c|c|c|c|c|c|}
\hline & N1 & $\mathrm{N} 2$ & N3 & $\mathrm{N} 4$ & S1 & S2 & S3 & IS & C & $\mathrm{VN}$ \\
\hline \multirow[t]{2}{*}{ NCEP } & $\begin{array}{l}\text { OD- } \\
\text { WD }\end{array}$ & $\downarrow$ & $\downarrow \downarrow$ & $\downarrow$ & $\leftrightarrow$ & $\uparrow$ & $\downarrow$ & $\leftrightarrow$ & $\uparrow$ & $\uparrow$ & $\downarrow$ \\
\hline & $\begin{array}{l}\text { WD- } \\
\text { END }\end{array}$ & $\uparrow$ & $\downarrow$ & $\downarrow \downarrow \downarrow$ & $\downarrow$ & $\uparrow$ & $\downarrow$ & $\uparrow$ & $\downarrow$ & $\downarrow$ & $\downarrow$ \\
\hline \multirow[t]{2}{*}{ JRA25 } & $\begin{array}{l}\text { OD- } \\
\text { WD }\end{array}$ & $\leftrightarrow$ & $\downarrow \downarrow$ & $\downarrow$ & $\downarrow$ & $\uparrow$ & $\downarrow$ & $\uparrow$ & $\uparrow$ & $\downarrow$ & $\downarrow$ \\
\hline & $\begin{array}{l}\text { WD- } \\
\text { END }\end{array}$ & $\leftrightarrow$ & $\leftrightarrow$ & $\leftrightarrow$ & $\downarrow \downarrow$ & $\uparrow$ & $\downarrow$ & $\uparrow$ & $\downarrow$ & $\downarrow$ & $\downarrow$ \\
\hline \multirow[t]{2}{*}{ JRA55 } & $\begin{array}{l}\text { OD- } \\
\text { WD }\end{array}$ & $\downarrow$ & $\downarrow \downarrow$ & $\downarrow$ & $\uparrow$ & $\uparrow$ & $\leftrightarrow$ & $\uparrow$ & $\uparrow$ & $\uparrow$ & $\downarrow$ \\
\hline & $\begin{array}{l}\text { WD- } \\
\text { END }\end{array}$ & $\downarrow$ & $\downarrow$ & $\downarrow$ & $\downarrow \downarrow \downarrow$ & $\uparrow$ & $\downarrow$ & $\uparrow$ & $\downarrow$ & $\downarrow$ & $\downarrow$ \\
\hline \multirow[t]{2}{*}{$\begin{array}{l}\text { ERA- } \\
\text { Interim }\end{array}$} & $\begin{array}{l}\text { OD- } \\
\text { WD }\end{array}$ & $\downarrow \downarrow \downarrow$ & $\downarrow \downarrow \downarrow \downarrow$ & $\downarrow \downarrow \downarrow$ & $\uparrow$ & $\uparrow$ & $\uparrow$ & $\leftrightarrow$ & $\uparrow$ & $\uparrow$ & $\downarrow$ \\
\hline & $\begin{array}{l}\text { WD- } \\
\text { END }\end{array}$ & $\downarrow$ & $\downarrow \downarrow \downarrow$ & $\downarrow \downarrow \downarrow$ & $\downarrow \downarrow \downarrow \downarrow$ & $\uparrow$ & $\downarrow$ & $\downarrow$ & $\downarrow$ & $\downarrow$ & $\downarrow$ \\
\hline
\end{tabular}

Table 6.3 Trends of changes of rainfall in monsoon (OD-WD) and post-monsoon (WD-END) period, discriminated by the NRM monsoon index, in each climate sub-region. Trend is identified by the Mann-Kendall test. The signs $\{\uparrow, \downarrow, \leftrightarrow\}$ denote the increase, decrease, or no trend. One arrow means that trend is not significant. Two, three, and four arrows represent $90 \%, 95 \%$ and $99 \%$ confidence interval for the existence of trend. N(1-3), S(1-3), IS, C, and VN represent climate subregions of northern $(\mathrm{N})$, southern(S), island (IS), Central (C), and Vietnam, respectively. OD, WD, END denote onset day, withdrawal day, and 30 November. 

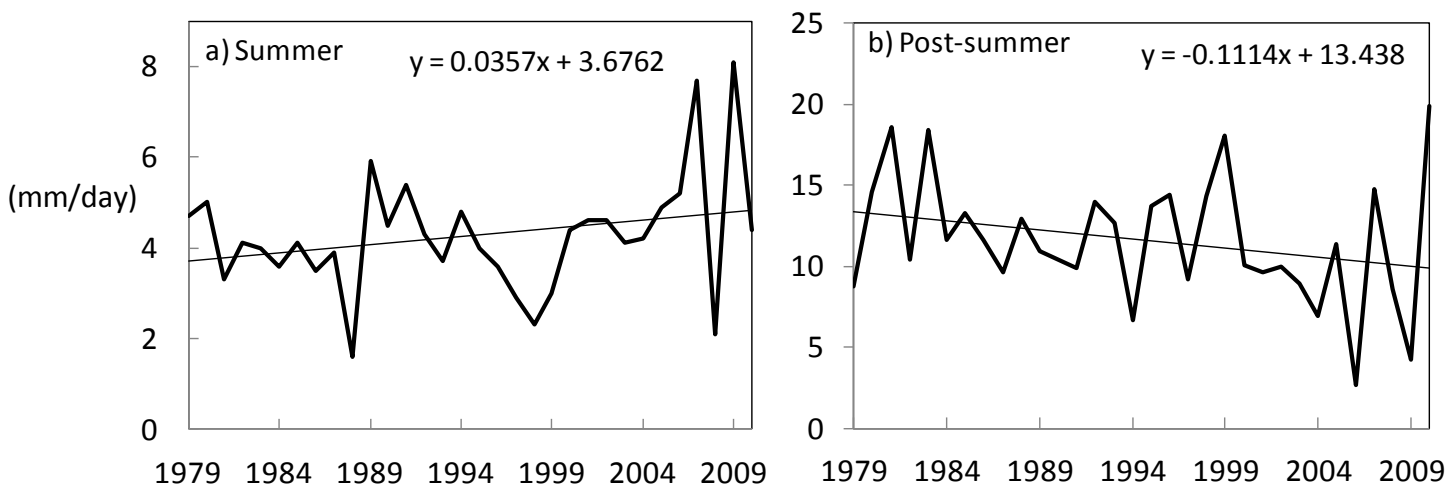

Figure 6.8. Vortex-induced rainfall in summer (a) and post-summer (b) season. Summer and postsummer are identified by the NRM index. Trend is identified by the least squared method. Y-axis: mm/day. This figure uses ERA-Interim reanalysis data set.

The trends found in these two methods are mostly consistent, particularly the decrease of 1) VR in post-summer in Central and the whole country, 2) VR in the whole year in the North, and as the result, 3) observation rainfall and VR in Vietnam. The decline of VR in the post-summer season may confirm the decrease of vortex activity in Vietnam, especially in Central Vietnam. This decrease can be regarded as the reduction of vortex intensity, vortex frequency, or both of these. This new result is stimulated studies in the future in subjects of studying the interannual variability of the semi-permanent vortex SEAMCV. 


\section{CHAPTER 7}

\section{DISCUSSIONS AND CONCLUSIONS}

Every new beginning comes from

some other beginning's end.

\section{Seneca}

Rainfall is investigated comprehensively in both spatial and temporal aspects in this study. Sixty stations, the most complete dataset, in terms of the number of observation stations and the length of the time-series, represented for all Vietnam climate sub-regions are used. The temporal separation (monsoon and post-monsoon) characterises the processes involved (monsoon and vortex) in rainfall climate in this country.

This Ph.D project is a journey which starts with the general climate in Vietnam, goes through the monsoon rainfall, and arrives finally at the vortex-induced rainfall. Along this journey, we recognise that the monsoon and tropical vortices are the two main determinates in Vietnam rainfall variability.

The climate in Vietnam over the past 40 years is characterised by the following key features: temperature increases; a decrease of rainfall in the whole country but marked by significant declines in the North; vortex-induced rainfall contributing up to $70-90 \%$ rainfall in Central regions; a increasing (decreasing) tendency of tropical vortex activity in the summer (post-summer) season is found in the Central Vietnam.

The temporal separation of rainfall is aided by the establishment of a unified monsoon index. The NRM index is used to determine the onset-withdrawal dates of the monsoon more consistently and precisely. Monsoon and post-monsoon periods can thus be studied separately. 
Rainfall in the post-monsoon period is associated with vortex activity. One of the successes of this project is the identification and description of the semi-permanent Southeast Asian Sea -Maritime Continent Vortex (SEAMCV). Expanding on previous understanding of the Borneo vortex (Chang et al., 2006), we see that the SEAMCV exists almost year-round, migrating from the coast of Vietnam - Southeast Asian Sea Philippines in summer to the Borneo Island region in winter. It is this vortex and tropical cyclones from the Northwest Pacific region that significantly contribute to the rainfall in SEASMC region.

Variability and trends in rainfall and temperature have been examined on both national and regional scales. An increase in annual average temperature of $0.26 \pm 0.10^{\circ} \mathrm{C}$ per decade was found over Vietnam; this is double the rate of global warming but is less than the 0.29 to $0.34^{\circ} \mathrm{C}$ per decade reported for global land-only stations in the Northern Hemisphere (IPCC, 2007). In most sub-regions, increasing trends of mean winter temperature are greater than those in summer by about $25-40 \%$, and as much as $70 \%$ in the Central coast S1 region. Except for the Central Highlands (S2), annual and seasonal average temperature in southern regions has increased more than in the north. Winter minimum temperatures have risen more than summer maximum temperatures in the South whilst the reverse pattern is observed in most of the Northern sub-regions.

In comparison to mean temperature where statistically significant trends were found in all sub-regions, precipitation showed statistically significant variability in only two subregions. These are N3 where decreasing precipitation is recorded and IS where it is increasing.

Nguyen and Nguyen (2004) defined the climate sub-regions of Vietnam based on their expert judgments of observational data. Here we use cluster analysis (Kidson, 1997; Huth, 2001) to determine the sub-regional climate boundaries for both rainfall and temperature. Comparisons gave a broad consistency between the two approaches for determining the climate sub-regions. Both analyses gave three sub-regions in the north $(\mathrm{N} 1, \mathrm{~N} 2, \mathrm{~N} 3)$ and two in the south $(\mathrm{S} 2, \mathrm{~S} 3)$. In the cluster analysis more than two sub-regions in the Central area of the country (N4 and S1 in the conventional classification) are found. This may reflect complex features of climate variables, especially rainfall, for this part of the 
country. We note that Central is the region most vulnerable to extreme rainfall caused by tropical vortex activity. The cluster analysis also revealed distinctly different climate characteristics for IS. This is not surprising as the IS station is about $400 \mathrm{~km}$ offshore in the Southeast Asian Sea.

The trend of annual average temperature anomaly in Vietnam is comparable with those in Northern Hemisphere oceans (IPCC 2007, Ar4 3.2.2), however, the mechanisms that cause the abnormally high of maximum daily temperatures at IS $\left(0.47^{\circ} \mathrm{C}\right.$ per decade) are unknown.

It has previously been demonstrated that ENSO activity is the principal large scale forcing of temperature and rainfall variability over the Western Pacific (Lau et al., 2000; Dai and Wigley, 2000; Caesar et al., 2011). This is also confirmed in this study. Here we also found that the ENSO linkage with climate variability at the sub-regional scale in Vietnam is latitude-dependent. Spatial correlation maps between rainfall anomalies in each sub-region and large scale variables (e.g. MSLP and SSTA) show that correlation coefficients vary from $0.0-0.2$ in the North to $0.4-0.5$ in the South.

Overall, it was found that temperatures are increasing in the whole country at both seasonal and annual time-scales. Meanwhile only two of the eight climate sub-regions show significant changes in rainfall. This result reflects the more complex characteristic of rainfall; it is this challenge that inspires us to go further onto the next part of this project. 
The interannual variability of rainfall climate of Vietnam is studied in depth through consideration of the length of the wet season. In the previous part of the study we investigated monthly rainfall variability, here rainfall variability is characterised by the onset-withdrawal dates (OD-WD) of the summer monsoon. A simple approach has been proposed to define the monsoon onset-withdrawal dates. The NRM index is a combination of standardised (5-day running average) mean sea level pressure and zonal wind at $850 \mathrm{hPa}$ with its sign taken from the wind component. To our knowledge, the NRM is the first fully general monsoon index which has been tested and can successfully detect OD-Peak-WD in monsoon regions worldwide. The current study took Vietnam and the Southeast Asian Sea region as an example to show the year-by-year OD-WD detection process. This process was successfully applied to all other global monsoon regions, a first for any monsoon index in the literature, as far as the author knows.

The two-variable NRM index was compared with the solely wind-based index of Wang et al. [2004]. Monsoon onset timing derived from the two approaches differed by only a few days in most cases. Where the two approaches differed significantly, this was due more to the limited time window used by Wang et al. [2004] rather than the use of a different index. It is well known that there is no one 'correct' way to define monsoon timing and no one preferred monsoon index in the literature (e.g. Wang et al. 2008 compared 25 different indices). Based on our analysis, it is not possible to say that the index defined here is 'better' than others in the literature in terms of defining monsoon onset timing. However, we argue that it is preferred because it represents both physical components of the monsoon, it can be applied universally around the globe, it allows determination of both onset and withdrawal dates, and it performs at least as well as other published monsoon indices.

The outcomes of this part (OD-WD basis) of study are not only in agreement with the results in the previous part (monthly basis), but also show clearer trends of changes in rainfall in most climate sub-regions. In monthly investigation, statistically significant trends were found only in N3 (decline) and IS (increase) sub-regions. Here, taking the ODWD period into consideration, we also found a decrease of rainfall at the national scale and an increase in the IS sub-region. Secondly, compared to finding only a decreasing trend in 
$\mathrm{N} 3$ rainfall in monthly investigations, here five out of eight climate sub-regions (N1, N2, N3, S1, S2) during the OD-WD period show the same decreasing trend as seen for N3 in monthly-basis investigations. Thirdly, the monsoon (post-monsoon) rainfall trend in Central has significantly risen (declined) in 1979-2010. Considering the high frequency of tropical cyclone activity affecting Central region year-round, these tendencies may suggest that Central Vietnam will witness the changes of tropical cyclone activity during the summer and winter monsoon in future.

The predictability of the monsoon rainfall can have a greater opportunity with the monsoon and non-monsoon discrimination. Over Vietnam and the Southeast Asian Sea region, the quantity of monsoon rainfall is found to be significantly correlated with monsoon onset dates OD. Since NRM is based on two rainfall-proxy variables, OD-WD can be estimated from predicted values of those proxies. In agricultural areas, knowing OD-WD and its amount of rainfall in advance would be extremely useful for planning cultivation. Currently Global Circulation Models can provide reliable seasonal forecasts in the tropics. Such products can potentially be used for the NRM index to estimate the onsetwithdrawal phases and the rainfall quantity of summer monsoon seasons.

In addition, ENSO's effect on monsoon rainfall can be studied through the NRM index. For example, in Table 5.2 (Chapter 5), El Niño years (1982, 1983, 1991, 1997, 2004) exhibited late OD while La Niña years (1985, 1988, 1995, 2000, 2008) exhibited early OD. Such relationships are the subject of further study.

Another piece of future work that can be carried out in a straightforward fashion is to take climate model projections (such as those in the Coupled Model Intercomparison Project Phase 5, CMIP5) and apply the NRM index to estimate future onset-withdrawal phases for each monsoon region. The post-summer rainfall, as the result, will be identified globally. Thus, the interannual variations of future global monsoon rainfall in the framework of climate change can be addressed. 
The temporal split of rainfall, through the NRM index, reveals a non-monsoon rainfall process in Central Vietnam. At the same time (post-summer period), tropical cyclone is observed in this region. The final part of the thesis deals with tropical vortex activity and its associated rainfall.

Many of the findings in the latter part of the thesis regard to the nature of the SEAMCV. The SEAMCV is present almost year round, first appearing in summer in coastal Vietnam, moving southward during autumn, reaching Singapore-Borneo in winter, and staying near the Borneo region into spring until a new yearly cycle comes again in summer. The occurrence frequency of the SEAMCV is highest in winter and summer, and least in spring and autumn. The annual variation of SEAMCV and the picture of and mechanism for the seasonal march of rainfall over the Southeast Asian Sea region, from central Vietnam, north Southeast Asia Sea, down to the Maritime Continent, is clear and consistent with the results of Chang et al. (2006) and those of Chen et al. (2012a).

Along with the demonstration of the SEAMCV behaviour, the interannual variability and the diagnosis of the quantitative contribution of vortex-induced rainfall are the major results of this part of the study. During post-summer 1979-2010, SEAMCV contributes up to $80 \%$ of total rainfall in Vietnam, and $70-90 \%$ of total rainfall in the Central region. This quantitative contribution is comparable to the result from Chen et al. (2012b) in which the tropical cyclone rainfall takes into account about $95 \%$ of the total rainfall, but is quite different to the others (Rodgers et al., 2000, about 20-30\% total rainfall over the South China Sea region; Kubota and Wang, 2009, not exceeding $60 \%$ of rainfall in a station in Taiwan).

The mechanism of the existence of SEAMCV is considered by the two different monsoons. In summer time (the southwesterly), SEAMCV can be formed by the interaction between the south-west entrainment flow and the monsoon trough/ITCZ (Gray, 1998). In winter time (the northeasterly), SEAMCV can be formed by the northeasterly interacting with the equatorial easterly wave (Riehl, 1948), or the monsoon trough (Cheang, 1977). 
The decreasing trends of post-summer vortex-induced rainfall are found in most subregions, suggesting post-summer tropical vortices activity, including SEAMCV and Western North Pacific tropical cyclones, has diminished in the last three decades in Vietnam. Meanwhile the increase vortex-induced rainfall (not significant) in Central during the summer monsoon period may imply the intensification of vortex activity, or the monsoon activity, or both these two processes in Central.

In terms of future work, several works can be implemented. Firstly, a study of cyclone genesis of the SEAMCV could be performed. How and when do those vortices develop and become organized into a tropical depression or a tropical cyclone? In the last 62 years (1952-2013) at least one tropical cyclone (TC) or depression (TD) was formed each year in the Southeast Asian Sea region (according to the IBTrACS-WMO data set), except in 1969 and 2006. These two years show no TC/TD events over the region. Years with more than five TCs/TDs are 1965 (7 TCs/TDs), (1983, 1997, 2002, 2009, 6 TCs/TDs), and (1958, 1961, 1966, 1970, 1973, 1984, 1990, 1994, 2001, 2008, 5TC/TDs). Recall that 2001 was also the year of typhoon Vamei (Chang et al. 2003). Although cyclone genesis associated with the SEAMCV is beyond the scope of this study, we suspect that studying the extreme years, including 1969 and 2006, may give some more insight into the thermodynamic aspects of the SEAMCV.

Secondly, the decreasing trends of post-summer vortex-induced rainfall inspire a new investigation on the interannual variability of the semi-permanent vortex SEAMCV. How ENSO does impact to SEAMCV track and intensity?

Thirdly, outcomes from this part of the project can be connected to other studies. It is well-known that rainfall over the Southeast Asian Sea - Maritime Continent, through associated latent heat release, plays a significant role in the global energy balance (e.g.: Ramage, 1968; Trenberth et al., 2000b), hence changes in the intensity or trajectory of the SEAMCV may affect the broader climate system. Juneng and Tangang (2010) showed the northward tendency of the SEAMCV in wintertime, and meanwhile Gong and Ho (2002) mentioned the weakening of the Siberian High since 1970s. Is there any connection between these results? We know that the Siberian High is one of the leading winter synoptic features which can profoundly affect cold surge activity in the East Asian region. 
It may be that a weakening winter monsoon circulation is associated with weak cold surges, i.e. cold surges which could not penetrate as far south as the Borneo Island; the SEAMCV, as a consequence, would have a tendency to reach and remain at higher latitudes over the Southeast Asian Sea. In other words, coastal regions on the Malaysia Peninsula, in Vietnam, and the Philippines may become more vulnerable to larger extreme rainfalls in association with climate change. This possibility will be investigated in future studies. 


\section{REFERENCES}

Allan, R., and T. Ansell (2006), A new globally complete monthly historical gridded mean sea level pressure dataset (HadSLP2): 1850-2004, J. Clim., 19, 5816-5842.

Altava-Ortiz, V., M. C. Llasat, E. Ferrari, A. Atencia, S. Beniamino (2011), Monthly rainfall changes in Central and Western Mediterranean basins, at the end of the $20^{\text {th }}$ and beginning of the $21^{\text {st }}$ centuries, Int. J. Climatol., 31: 1943-1958.

Ananthakrishnan, R., J. M. Pathan, and S. S. Aralikatti (1981), On the northward advance of the ITCZ and the onset of the southwest monsoon rains over the southeast bay of Bengal, Int. J. Climatol., 1, 153-165.

Bretherton, C. S., C. Smith, and J. M. Wallace (1992), An intercomparison of methods for finding coupled patterns in climate data, J. Clim., 5, 541-560.

Bruzon, E., and P. Carton (1930), Le Climat de 1'Indochine et les Typhoons de la Mer de Chine. Exposition coloniale International Paris 1931, 310pp.

Burkey J. (2011), Seasonal Kendall Test with Slope for Serial Dependent Data. http://www.mathworks.com/matlabcentral/fileexchanges/22389-seasonal-kendall.

Cook, K. H., G. A. Meehl, and J. M. Arblaster (2012), Monsoon regimes and processes in CCSM4. Part II: African and American monsoon systems, J. Clim., 25, 2609-2621.

Caesar, J., L. V. Alexander, B. Trewin, K. Tse-ring, L. Sorany, V. Vuniyayawa, N. Keosavang, A. Shimana, M. M. Htay, J. Karmacharya, D. A. Jayasinghearachchi, J. Sakkamar, E. Soares, L. T. Hung, L. T. Thuong, C. T. Hue, N. T. T. Dung, P. V. Hung, H. D. Cuong, N. M. Cuong, S. Sirabaha (2011), Changes in temperature and precipitation extremes over the Indo-Pacific region from 1971 to 2005, Int. J. Climatol., 31, 791-801. 
Chang, C. -P., C. -H. Liu, and H. -C. Kuo (2003), Typhoon Vamei: An equatorial tropical cyclone formation, Geophys. Res. Lett., 30, 1150.

Chang, C. -P., J. E. Erickson, and K. M. Lau (1979), Northerly cold surges and nearequatorial disturbances over the winter MONEX area during December 1974, Part I: Synoptic aspects, Mon. Wea. Rev., 107, 812-829.

Chang, C. -P., P. A. Harr, and H. J. Chen (2005), Synoptic disturbances over the equatorial Southeast Asian Sea and western Maritime Continent during boreal winter, Mon. Wea. Rev., 133, 489-503.

Chang, C. -P., Z. Wang, and H. Hendon (2006), The Asian winter monsoon. The Asian Monsoon (Chap. 3), B. Wang, Ed., ISBN 3-540-40610-7 Springer-Verlag, 89-127.

Chang, C.-P., Y. Lei, C.-H. Sui, X. Lin, and F. Ren (2012), Tropical cyclone and extreme rainfall trends in East Asian summer monsoon since mid-20 ${ }^{\text {th }}$ century, Geophys. Res. Lett., 39, L1870, doi: 10.1029/2012GL052945.

Cheang, B. K. (1977), Synoptic features and structures of some equatorial vortices over the Southeast Asian Sea in the Malaysian region during the winter monsoon of December 1973, Pure Appl. Geophys., 115, 1303-1333.

Chen, T. C. (2002), A North-Pacific Short-wave Train during the Extremes Phases of ENSO, J. Clim., 15, 2359-2376.

Chen, T.-C., J.-D. Tsay, M.-C. Yen, and J. Matsumoto (2012a), The Winter Rainfall of Malaysia, J. Clim., 26, 936-958.

Chen, T.-C., J.-D. Tsay, M.-C. Yen, and J. Matsumoto (2012b), Interannual Variation of the Late Fall Rainfall in Central Vietnam, J. Clim., 25, 392-413. 
Chen, T. C., and J. H. Yoon (2000), Interannual Variation in Indochina Summer Monsoon Rainfall: Possible Mechanism, J. Clim., 13, 1979-1986. DOI:10.1175/15200442(2000)013<1979:IVIISM>2.0.CO;2.

Chowdary, J. S., S. P. Xie, H. Tokinaga, Y. M. Okumura, H. Kubota, N. Johnson, X. T. Zheng (2012), Interdecadal Variations in ENSO Teleconnection to the Indo-Western Pacific for 1870-2007, J. Clim, 25, 1722-1744. doi: http://dx.doi.org/10.1175/JCLI-D-11$\underline{00070.1}$

Christy, J. R., B. N. William, T. M. Richard (2009), Surface Temperature Variations in East Africa and Possible Causes, J. Clim, 22, 3342-3356. DOI: 10.1175/2008JCLI2726.1 http://dx.doi.org/10.1175/2009JCLI2551.1

Churchill, D. D., and R. A. Houze, Jr. (1984), Development and structure of winter monsoon cloud clusters on 10 December 1978, J. Atmos. Sci., 41, 933-960.

Collins, J. M., R. C. Rosane, M. Valdo da Silva (2009), Temperature Variability over South America, J. Clim., 22, 5854-5869.

Dai, A., T. R. Kark, B. Sun, K. E., Trenberth (2006), Recent trends in cloudiness over the United States: A Tale of Monitoring inadequacies, Bull. Amer. Meteor. Soc, 87(5), 597606. DOI:10.1175/BAMS-87-5-597.

Dai, A., T. M. Wigley (2000), Global Patterns of ENSO-induced Precipitation, Geophys. Res. Lett., 27(9),1283-1286.

Dai, A., K. E. Trenberth (1999), Effects of Clouds, Soil Moisture, Precipitation, and Water Vapor on Diurnal Temperature Range, J. Clim., 12, 2451-2473.

Dai, A., I. Y. Fung, A. D. D. Genio (1997), Surface Observed Global Land Precipitation Variations during 1900-88, J. Clim, 10, 2943-2962. 
Dare, R. A. (2013), Seasonal Tropical Cyclone Rain Volumes over Australia, J. Clim, 26, 5958-5964.

Dee, D. P., S. M. Uppala, A. J. Simmons, P. Berrisford, Poli, P., Kobayashi, S., Andrae, U., Balmaseda, M. A., Balsamo, G., Bauer, P., Bechtold, P., Beljaars, A. C. M., van de Berg, L., Bidlot, J., Bormann, N., Delsol, C., Dragani, R., Fuentes, M., Geer, A. J., Haimberger, L., Healy, S. B., Hersbach, H., Hólm, E. V., Isaksen, L., Kållberg, P., Köhler, M., Matricardi, M., McNally, A. P., Monge-Sanz, B. M., Morcrette, J.-J., Park, B.-K., Peubey, C., de Rosnay, P., Tavolato, C., Thépaut, J.-N. and Vitart, F. (2011), The ERAInterim reanalysis: configuration and performance of the data assimilation system, Q.J.R. Meteorol. Soc., 137, 553-597. doi: 10.1002/qj.828

Durre, I., and J. M. Wallace (2001), Factors Influencing the Cold-Season Diurnal Temperature Range in the United States, J. Clim, 14, 3263-3278.

Ebita, A., Kobayashi, S., Ota, Y., Moriya, M., Kumabe, R., Onogi, K., Harada, Y., Yasui, S., Miyaoka, K., Takahashi, K., Kamahori, H., Kobayashi, C., Endo, C., Soma, M., Oikawa, Y., and Ishimizu, T. (2011), The Japanese 55-year Reanalysis "JRA-55": an interim report, SOLA, 7, 149-152.

Endo, N., J. Matsumoto, and T. Lwin (2009), Trends in Precipitation Extremes over Southeast Asia, SOLA, 5, 168-171. doi:10.2151/sola.2009-043

Fan, Z. X., A. Bräuning, A. Thomas, J. B. Li, K. F. Cao (2011), Spatial and temporal temperature trends on the Yunnan Plateau (Southwest China) during 1961-2004, Intl. J. Climatol., 31, 2078-2090. DOI: 10.1002/joc.2214.

Garcia, S., and M. T. Kayano (2011), Some considerations on onset dates of the rainy season in Western-Central Brazil with antisymmetric outgoing longwave radiation relative to the equator, Int. J. Climatol., 33, 188-198. 
Gilbert, R. O. (1987), Statistical methods for environmental pollution monitoring. John Wiley \& Sons, Inc., New York. Pp 336. ISBN 0-471-28878-0.

Gong, D. Y., and Ho, C. H. (2002), The Siberian High and climate change over middle to high latitude Asia, Theor. App. Clim., 72, 1-9.

Goswami, B. N., B. Krishnamurthy, and H. Annamalai (1999), A broad-scale circulation index for the interannual variability of the Indian summer monsoon, Q. J. R. Meteorol. Soc., 125, 611-633.

Gray, W. M. (1998), The Formation of Tropical Cyclones, Meteorol. Atmos. Phys., 67, 3769.

Griffiths, G. M., Chambers LE, Haylock MR, Manton MJ, Nicholls N, Baek HJ, Choi Y, Della-Marta PM, Gosai A, Iga N, Lata R, Laurent V, Maitrepierre L, Nakamigawa H, Outprasitwong N, Solofa D, Tahani L, Thuy DT, Tibig L, Trewin B, Vediapan K, Zhai P. (2005), Change in mean temperature as a predictor of extreme temperature change in the Asia-Pacific region, Int. J. Clim., 25, 1301-1330.

Hattori, M., K. Tsuboki, and S. Mori (2010), Contribution of tropical cyclones to the seasonal change patterns of precipitation in the western North Pacific: Estimation based on JRA-25/ JCDAS, SOLA, 6, 101-104.

Hannanchi, A., Jolliffe, I. T., and Stephenson, D. B. (2007), Review Empirical orthogonal functions and related techniques in atmospheric science: A review, Intl. J. Climatol., 27, 1119-1152, doi: 10.1002/joc.1499.

Houze, R. A. Jr., S. G. Geotis, F. D. Marks, and A. K. West (1981), Winter monsoon convection in the vicinity of North Borneo, Part I: Structure and time variation of the clouds and precipitation, Mon. Wea. Rev., 109, 1595-1614. 
Hung, C.-W., and M. Yanai (2004), Factors contributing to the onset of the Australian summer monsoon, Q. J. R. Meteorol. Soc., 130, 739-758.

Huth, R. (2001), Disaggregating Climatic Trends by Classification of Circulation Patterns, Int. J. Climatol, 21, 135-153.

IPCC (2007), Climate change 2007: The physical science basis. Working group I Contribution to the Fourth Assessment Report of the Intergovernmental Panel on Climate Change. Summary for Policymakers, Technical Summary and Frequently Asked Questions. Pp 142, Cambridge University Press, New York. ISBN 92-9169-121-6.

IPCC (2013), Climate Change 2013: The Physical Science Basis. Contribution of Working Group I to the Fifth Assessment Report of the Intergovernmental Panel on Climate Change. [Stocker, T.F., D. Qin, G.-K. Plattner, M. Tignor, S.K. Allen, J. Boschung, A. Nauels, Y. Xia, V. Bex and P.M. Midgley (eds.)], Cambridge University Press, Cambridge, United Kingdom and New York, NY, USA, 1535 pp, doi:10.1017/CBO9781107415324.

Jhajharia, D., V. P. Singh (2011), Trends in temperature, diurnal temperature range and sunshine duration in Northeast India, Int. J. Climatol., 31, 1353-1367. DOI: 10.1002/joc.2164.

Jolliffe, I.T. (2002), Principal Component Analysis, 2nd edn. Springer: New York. Jones, P. D., K. E. Trenberth, P. G. Ambenje, R. Bojariu, Easterling DR, Klein TG, Parker DE, Renwick JA, Rahimzadeh F, Rusticucci MM, and Coauthors (2007), Climate Change 2007: The Physical Science Basis (Chapter 3). Observations: Surface and Atmospheric Climate Change. Contribution of Working Group I to the Fourth Assessment Report of the Intergovernmental Panel on Climate Change: 235-336.

Johnson, R.H., and R. A. Houze (1987), Precipitating cloud systems of the Asian monsoon, Monsoon Meteorology, C.-P. Chang and T. N. Krishnamurti, Eds., Oxford University Press, 298-353. 
Jones, P. D., K. E., Trenberth, P. G., Ambenje, R. Bojariu, D. R. Easterling, T. G. Klein, D. E. Parker, J. A. Renwick, J. Rusticucci, B. Soden, P. Zhai, S. Solomon, D. Qin, M. Manning, Z. Chen, M. Marquis, K. B. Averyt, M. Tignor, H. L. Miller (eds.) (2007), Observations: Surface and Atmospheric Climate Change. In: Climate Change 2007: The Physical Science Basis. Contribution of Working Group I to the Fourth Assessment Report of the Intergovernmental Panel on Climate Change. Cambridge University Press, Cambridge, United Kingdom and New York, NY, USA, pp. 235-336.

Juneng, L. and F. T. Tangang (2010), Long-term trends of winter monsoon synoptic circulations over Maritime Continent: 1962-2007, Atmos. Sci. Let., 11, 199-203.

Kidson, J. W. (1997), The utility of surface and upper air data in synoptic climatological specification of surface climatic variables, Int. J. Climatol., 17, 399-413.

Kidson, J. W. (2000), An analysis of New Zealand synoptic types and their use in defining weather regimes, Int. J. Climatol., 20,299-316. doi: 10.1002/(SICI)10970088(20000315)20:3<299::AID-JOC474>3.0.CO;2-B

Klingbjer, P., A. Moberg (2003), A composite monthly temperature record from Tornedalen in northern Sweden, 1802-2002, Int. J. Climatol., 23, 1465-1494. DOI: 10.1002/joc.946.

Knapp, K. R., M. C. Kruk, D. H. Levinson, H. J Diamond, and C. J. Neumann (2010), The International Best Track Archive for Climate Stewardship (IBTrACS). Bull. Amer. Meteor. Soc., 91, 363-376, DOI: 10.1175/2009BAMS2755.1.

Kubota, H. and B. Wang (2009), How much Do Tropical Cyclones Affects Seasonal and Interannual Rainfall Variability over the Western North Pacific? J. Clim., 22, 5495-5510. Kueh, M. T., and S. C. Lin (2010), A climatological study on the role of the South China Sea monsoon onset in the development of the East Asian summer monsoon, Theor. Appl. Clim. J., 99, 163-186. 
Kueh, M. T., and S. C. Lin (2010), A climatological study on the role of the South China Sea monsoon onset in the development of the East Asian summer monsoon, Theor. Appl. Clim. J., 99, 163-186.

Lau, K. M., K. M. Kim, S. Yang (2000), Dynamical and Boundary Forcing Characteristics of Regional Components of the Asian Summer Monsoon, J. Clim., 13, 2461-2482.

Lau, K. M., and S. Yang (1997), Climatology and interannual variability of the Southeast Asian summer monsoon, Adv. Atmos. Sci., 14, 141-161.

Lavers, D., C. Prudhomme, and D. M. Hannah (2013), European precipitation connections with large-scale mean sea-level pressure (MSLP) fields, Hydrol. Sci. J., 58(2), 310-327.

Li, C., and M. Yanai (1996), The onset and interannual variability of the Asian summer monsoon in relation to land-sea thermal contrast, J. Clim., 9, 358-375.

Li, J., and Q. Zeng (2002), A unified monsoon index, Geophys. Res. Lett., 29(8), 1274, doi:10.1029/2001GL013874.

Liebmann, B., I. Bladé, N. A. Bond, D. Gochis, D. Allured, and G. T. Bates (2008), Characteristics of north American summertime rainfall with emphasis on the monsoon, $J$. Clim., 21, 1277-1294.

Lotsch, A. (2003), Coupled vegetation-precipitation variability observed from satellite and climate records, Geophys. Res. Lett., 30, 14, 1774.

Mann, H. B. (1945), Non-parametric tests against trend, Econometrica, 13, 245-259.

Manton, M. J., P. M. Della-Marta, M. R. Haylock, K. J. Hennessy, N. Nicholls, L. E. Chambers, D. A. Collins, G. Daw, A. Finet, D. Gunawan, K. Inape, H. Isobe, T. S. Kestin, P. Lefale, C. H. Leyu, T. Lwin, L. Maitrepierre, N. Ouprasitwong, C. M. Page, J. Pahalad, 
N. Plummer, M. J. Salinger, R. Suppiah, V. L. TranL, B. Trewin, L. Tibig, D. Yee (2001), Trends in extreme daily rainfall and temperature in southeast asia and the south pacific: 1961-1998, Int. J. Clim., 21, 269-284.

Mao, J., and J. C. L. Chan (2004), Relationship between the onset of the South China Sea Summer Monsoon and the structure of the Asian subtropical anticyclone, J. Meteor. Soc. Japan, 82, 3, 845-849.

Marks, F. D., G. Kappler, and M. DeMaria (2002), Development of a tropical cyclone rainfall climatology and persistence (RCLIPER) model. Preprints, 25th Conf. on Hurricanes and Tropical Meteorology, San Diego, CA, Amer. Meteor. Soc.,327-328.

Matsumoto, J. (1997), Seasonal transition of summer rainy season over Indochina and adjacent monsoon region, Adv. Atmos. Sci, 14, 231-245.

Martínez, M. D., C. Serra, A. Burgueño, X. Lana (2010), Time trends of daily maximum and minimum temperatures in Catalonia (ne Spain) for the period 1975-2004. Int. $J$.

Climatol., 30, 267-290. DOI: 10.1002/joc.1884.

McPhaden, M. J., et al. (2008), RAMA: Research Moored Array for African-AsianAustralian Monsoon Analysis and Prediction, Bull. Am. Meteorol. Soc., 90, 459-480.

Meehl, G. A. (1987), The annual cycle and interannual variability in the tropical pacific and indian ocean regions, Mon. Wea. Rev., 115, 27-50. DOI: 10.1175/15200493(1987)115<0027:TACAIV>2.0.CO;2.

Moberg, A., Jones PD, Lister DH, Walther A, Brunet M, and Coauthors. (2006), Indices for daily temperature and precipitation extremes in Europe analyses for the period 19012000, J. Geophys. Res., 111, D22106. 
Mooley, D. A., and B. Parthasarathy (1984), Fluctuations in all-India summer monsoon rainfall during 1871-1978, Clim. Change, 6, 287-301.

Nandintsetseg, B., Greene JS, Goulden CE. (2007), Trends in extreme daily precipitation and temperature near lake Hövsgöl, Mongolia, Int. J. Climatol., 27, 341-347. DOI: 10.1002/joc. 1404 .

NCHMF (2009), Medium range Weather Forecast Regulation of National Centre of Hydrometeorological Forecastings. Pp30 (Vietnamese language).

Nguyen, D. N., and T. H. Nguyen (2004), Climate and Climate resources in Vietnam. Agricultural Publishing House, Pp 296. (Vietnamese language).

Nguyen, D. Q., J. Renwick, and J. McGregor (2014a). Variation of surface temperature and rainfall in Viet Nam from 1971 to 2010, Int. J. Climatol., 34, 249-264, DOI: 10.1002/joc.3684.

Nguyen, D. Q., J. Renwick, and J. McGregor (2014b). Variations of Monsoon Rainfall: A Simple Unified Index, Geophys. Res. Lett., 41, 575-581, DOI: 10.1002/2013GL058155.

Nguyen, D. T., C. Uvo, D. Rosbjerg (2007), Short Communication: Relationship between the tropical Pacific and Indian Ocean sea-surface temperature and monthly precipitation over the central highlands, Vietnam, Int. J. Climatol., 27, 1439-1454. DOI: 10.1002/joc. 1486 .

Nguyen, K. C., and K. J. E. Walsh (2001), Interannual, decadal, and transient greenhouse simulation of tropical cyclone-like vortices in a regional climate model of the South Pacific, J. Clim.,14, 3043-3054.

Onogi, K., Tsutsui J, Koide H, Sakamoto M, Kobayashi S, Hatsushika H, Matsumoto T, and Coauthors. (2007), The JRA-25 Reanalysis, J. Meteor. Soc. Japan, 85, 369-432. 
Page, C. M., N. Nicholls, N. Plummer, B. Trewin, M. Manton, L. Alexander, L. E. Chambers, Y. Choi, D. A. Collins, A. Gosai, P. Della-Marta, M. R. Haylock, K. Inapa, V. Laurent, L. Maitrepiere, E. E. P. Marmur, H. Nakamigawa, N. Ouprasitwong, S. McGree, J. Pahalad, M. J. Salinger, L. Tibig, T. D. Tran, K. Vediapan, P. Zhai (2004), Data Rescue in the Southeast Asia and South Pacific Region: Challenges and Opportunities, Insights and Innovations, 1483-1489.

Pham, X. T., B. Fontaine, and N. Philippon (2010), Onset of the summer monsoon over the Southern Vietnam and its predictability, Theor. Appl. Clim. J., 99, 105-113.

Pope, M., C. Jakob, and M. J. Reeder (2009), Regimes of the North Australian wet season, J. Clim., 9, 6699-6715.

Preisendorfer, R. W. (1988), Principal component analysis in meteorology and oceanography, Elsevier Pub. Co., N. Y., 425pp.

Qian, W., and D. K. Lee (2000), Seasonal March of Asian summer monsoon, Int. J. Climatol., 20, 1371-1386.

Ramage, C.S (1968), Role of a tropical "Maritime Continent" in the Atmospheric Circulation, Mon. Wea.Rev., 96, 365-370.

Renwick, J.A., and J. M. Wallace (1995), Predictable anomaly patterns and the forecast skill of Northern Hemisphere wintertime 500-mb height fields, Mon. Wea. Rev., 123(7), 2114-2131.

Riehl, H. (1948), On the formation of typhoons, J. Meteor., 5, No. 6, 247-264.

Rodgers, E.B., R. F. Adler, and H. F. Pierce (2000), Contribution of Tropical Cyclones to the North Pacific Climatological Rainfall as Observed from Satellites, J. App. Meteor., 39, 1658-1678. 
Sadler, J. C. (1967), On the origin of tropical vortices. Working Panel on Trop. Dyn. Meteor. Naval Post Graduate School, Monterey, CA, 39-75.

Santer, B. D., P. W. Thorne, L. Haimberger, K. E. Taylor, T. M. L. Wigley, J. R. Lanzante, S. Solomon, M. Free, P. J. Gleckler, P. D. Jones, T. R. Karl, S. A. Klein, C. Mears, D. Nychka, G. A. Schmidt, S. C. Sherwood, F. J. Wentz (2008), Consistency of modelled and observed temperature trends in the tropical troposphere, Int. J. Climatol., 28: 1703-1722. DOI: $10.1002 /$ joc. 1756.

Seleshi, Y., and U. Zanke (2004), Recent changes in rainfall and rainy days in Ethiopia, Int. J. Climatol., 24, 973-983.

Sen, P. K. (1968), Estimates of the regression coefficient based on Kendall's tau, J. Amer. Stat. Assoc., 63, 1379-1389.

Tangang, F. T., E. Salimun, L. Juneng, P. N. Vinayachandran, K. S. Yap, C. J. C. Reason, S. K. Behera and T. Yasunari (2008), On the roles of northeast cold surge, the Borneo vortex, the Madden-Julian Oscillation and the Indian Ocean Dipole during the worst 2006/2007 flood in Peninsular Malaysia, Geophy. Res. Lett., 35, L14S07. DOI:10.1029/2008GL033429.

Trenberth, K. E., and J. M. Caron (2000a), The Southern oscillation revisited: Sea level pressure, surface temperatures, J. Clim., 13, 4358-4365.

Trenberth, K. E., P. S. David, and M. C. Julie (2000b), The Global Monsoon as Seen through the Divergent Atmospheric Circulation, J. Clim., 13, 3969-3993. DOI: 10.1175/1520-0442(2000)013<3969:TGMAST>2.0.CO;2 
Trenberth, K. E. , W. H. James, and P. S. David (2006), The Asian monsoon: Global perspectives. The Asian Monsoon. Chapter 2. p.67-87. Praxis, Springer Berlin Heidelberg publisher.

Von Storch, H., and A. Navarra (1999), Analysis of Climate Variability. Applications of statistical techniques. $2^{\text {nd }}$ Edition. Springer-Verlag Publishing Co., Berlin, pp342.

Walker, G. T., and E. W. Bliss (1932), World Weather V, Mem. R. Meteorol. Soc., 4, 5384.

Wallace, J. M., C. Smith and C. S. Bretherton (1992), Singular value decomposition of wintertime sea surface temperature and $500 \mathrm{mb}$ height anomalies, J. Climate, 5, 561-576.

Wang, B. (2006), The Asian Monsoon, Springer/Praxis Publishing Co., New York, pp787. Wang, B., and LinHo (2002), Rainy season of the Asian-Pacific summer monsoon, $J$. Clim., 15, 386-398.

Wang, B., L. Ho, Y. Z. , and M.-M. Lu (2004), Definition of South China Sea monsoon onset and commencement of the East Asia summer monsoon, J. Clim., 17, 699-710.

Wang, B., and Z. Fan (1999), Choice of South Asian summer monsoon indices, Bull. Am. Meteorol. Soc., 80, 629-638.

Wang, B., R. Wu, and X. Fu (2000), Pacific-East Asian Teleconnection: How Does ENSO Affect East Asian Climate? J. Clim., 13, 1517-1536.

Wang, B., Z. Wu, J. Li, J. Liu, C.-P. Chang, Y. Ding, and G. Wu (2008), How to measure the strength of the East Asian summer monsoon, J. Clim., 21, 4449-4463.

Webster, P. J., and S. Yang (1992), Monsoon and ENSO: Selectively interactive systems, Q. J. R. Meteorol. Soc., 118, 877-926. 
Wettstein, J. J., and L. O. Mearns (2002), The Influence of the North Atlantic-Arctic Oscillation on Mean, Variance, and Extremes of Temperature in the Northeastern United States and Canada, J. Clim., 15, 3586-3600.

Wilks, D. S. (2011), Statistical Methods in the Atmospheric Sciences, $3^{\text {rd }}$ edn, Academic Press, pp704.

Xie, S. P., Q. Xie, D. Wang, W. T. Liu (2003), Summer upwelling in the South China Sea and its role in regional climate variations, J. Geophys. Res., 108(C8): 3261. DOI:

10.1029/2003 JC001867.

Xie, P., and P. A. Arkin (1997), Global precipitation: A 17- year monthly analysis based on gauge observations, satellite estimates, and numerical model outputs, Bull. Amer. Meteor. Soc.,78, 2539-2558.

Yatagai, A., K. Kamiguchi, O. Arakawa, A. Hamada, N. Yasutomi, and A. Kitoh (2012), APHRODITE: Constructing a Long-Term Daily Gridded Precipitation Dataset or Asia Based on a Dense Network of Rain Gauges, Bull. Amer. Meteor. Soc., 93, 1401-1415. doi: http://dx.doi.org/10.1175/BAMS-D-11-00122.1

Yen, M.-C., T. C. Chen, H.-L. Hu, R.-Y. Tzeng, D. T. Dinh, T. T. T. Nguyen, and C. J. Wong (2011), Interranual Variation of the Fall Rainfall in Central Vietnam, J. Met. Soc. Japan, 89A, 259-270.

Yokoi, S., T. Satomura, and J. Matsumoto (2007), Climatological Characteristics of the Intraseasonal Variation of Precipitation over the Indochina Peninsula, J. Clim., 20, 53015315. DOI: 10.1175/2007JCLI1357.1.

Yokoi, S., and J. Matsumoto (2008), Collaborative Effects of Cold Surge and Tropical Depression-Type Disturbance on Heavy rainfall in Central Vietnam, Mon. Wea. Rev., 136, 3275-3287. 
Zhang, S., and B. Wang (2008), Global summer monsoon rainy seasons, Int. J. Climatol., 28, 1563-1578.

Zhang, Y., K. R. Sperber, and J. S. Boyle (1997), Climatology and Interranual Variation of the East Asian Winter Monsoon: Results from the 1979-95 NCEP/NCAR Reanalysis, Mon. Wea. Rev., 125, 2605-2619.

Zhang, Z., J. C. Chan, and Y. Ding (2004), Characteristics, evolution and mechanisms of the summer monsoon onset over Southeast Asia, Int. J. Clim., 24, 1461-1482. 


\section{APPENDICES}

\section{Appendix for figures (AF)}
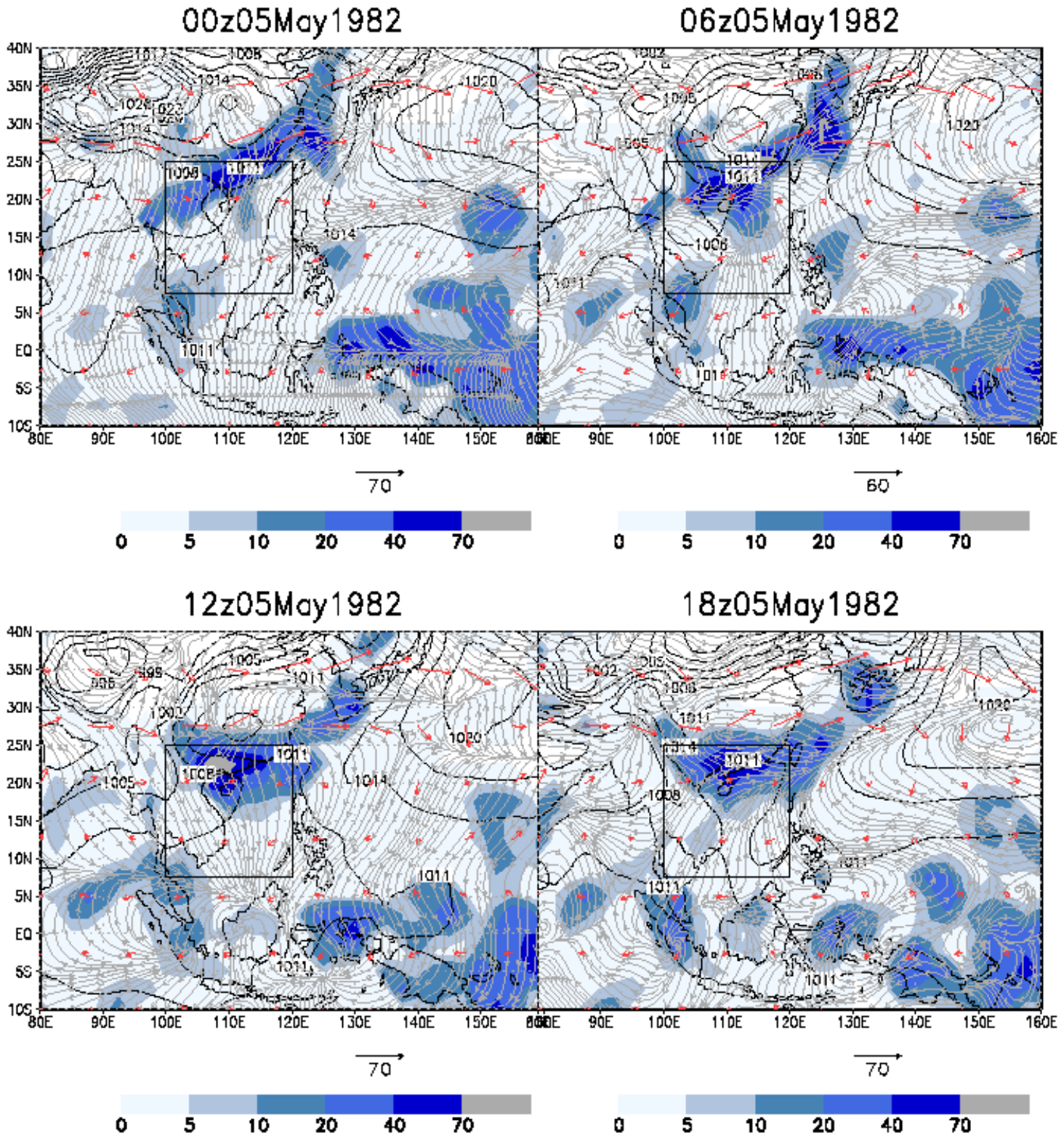

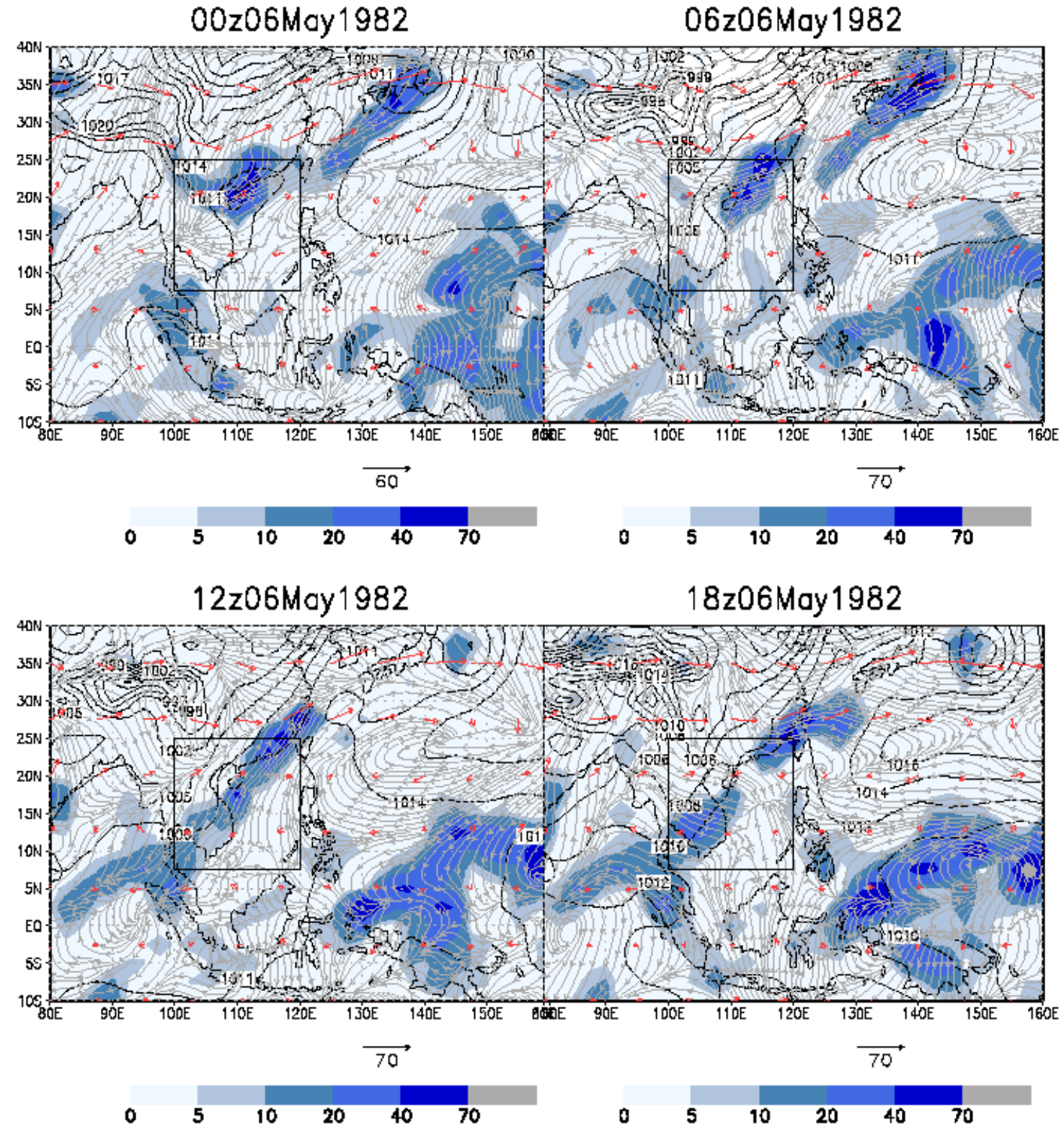


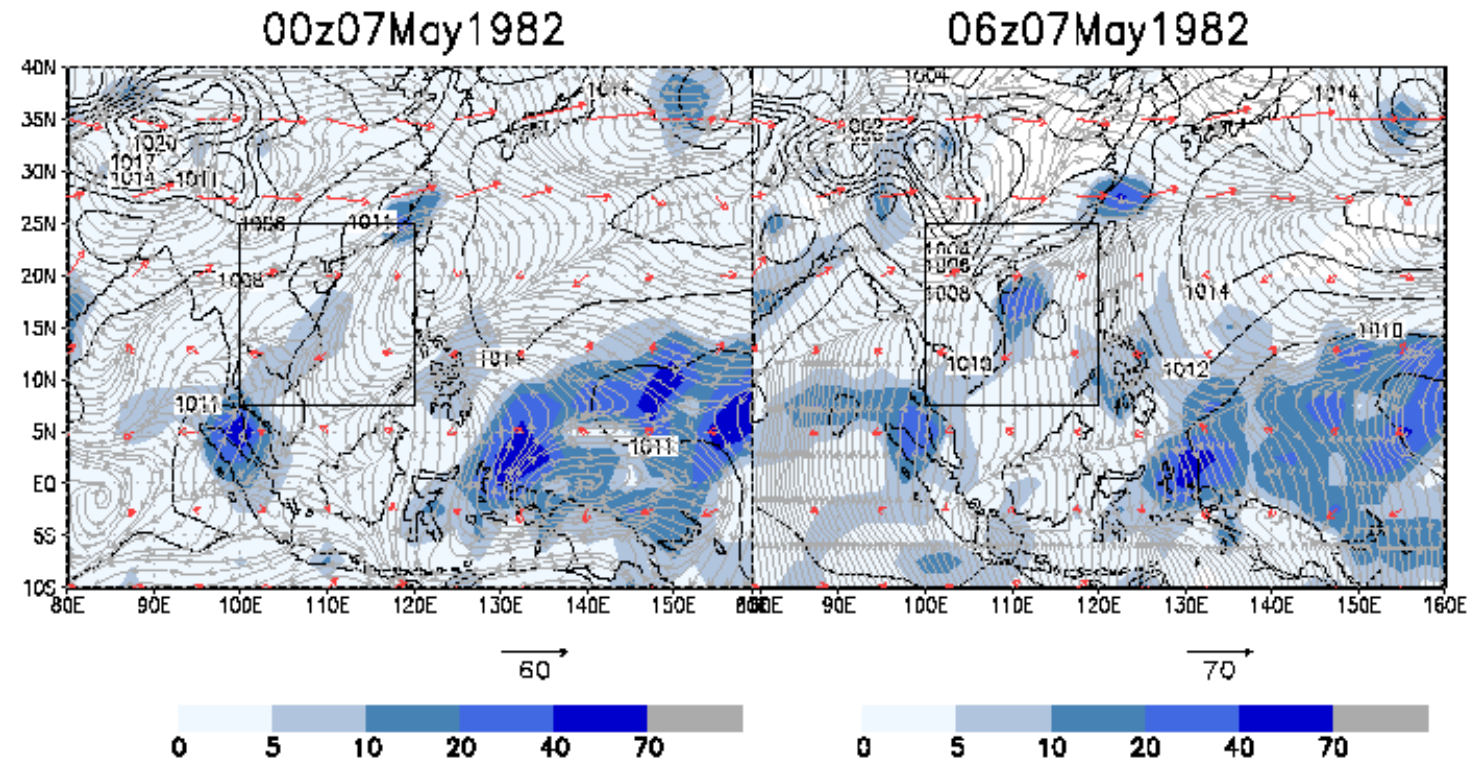

Figure AF1. Synoptic analysis map of the heavy rain episode on 5-6 May 1982 in Vietnam. Black contours denote Mean Sea Level Pressure (MSLP); gray contours: streamlines at $850 \mathrm{hPa}$; red arrows: wind direction at $200 \mathrm{hPa}$; shaded areas: rainfall (mm/day). JRA-25 reanalysis. 


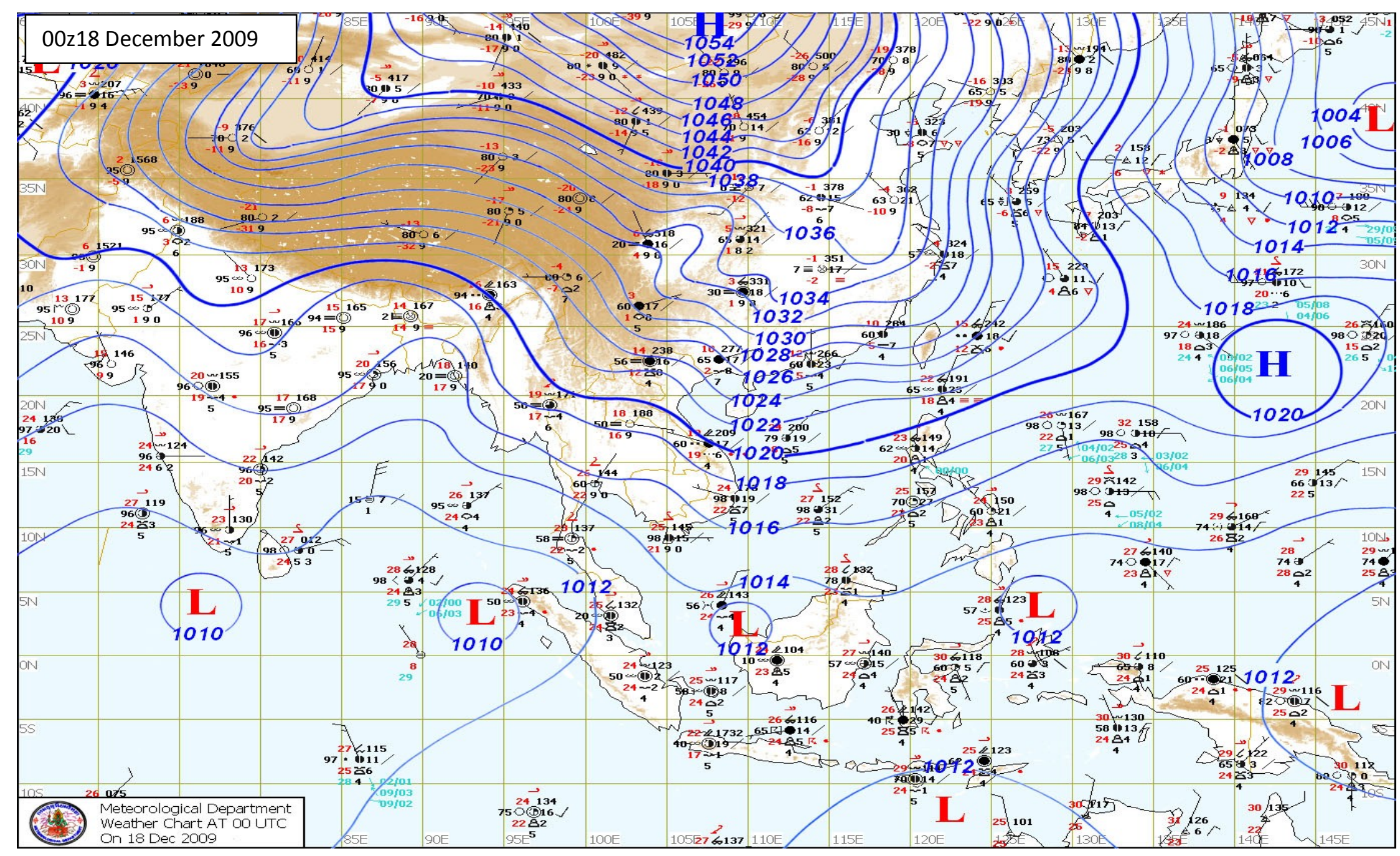

Figure AF2. TMD's surface analysis map from 00UTC 15December to 00UTC 18December 2009: A typical cold surge hitting Vietnam and the SEAS region. 

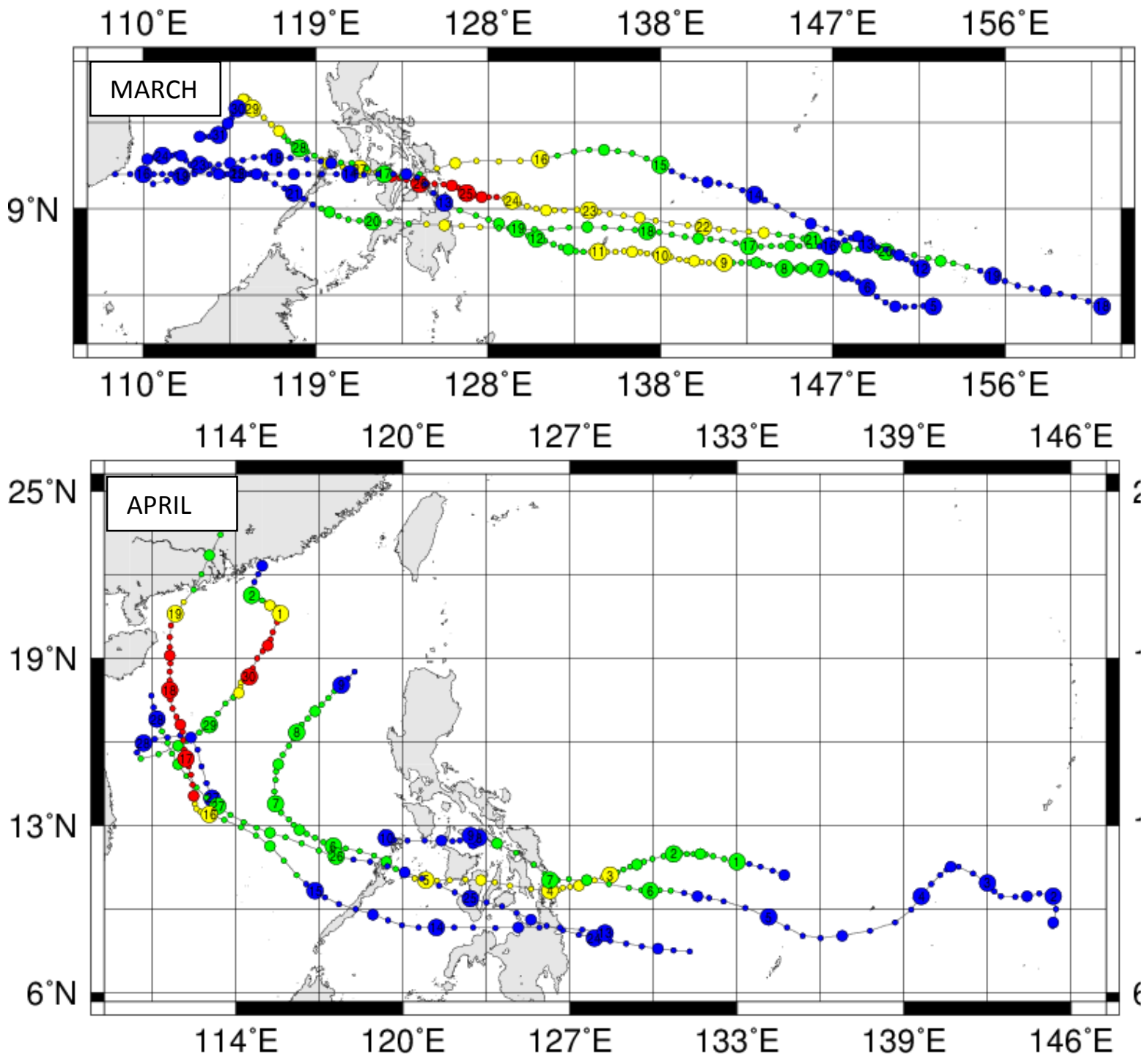

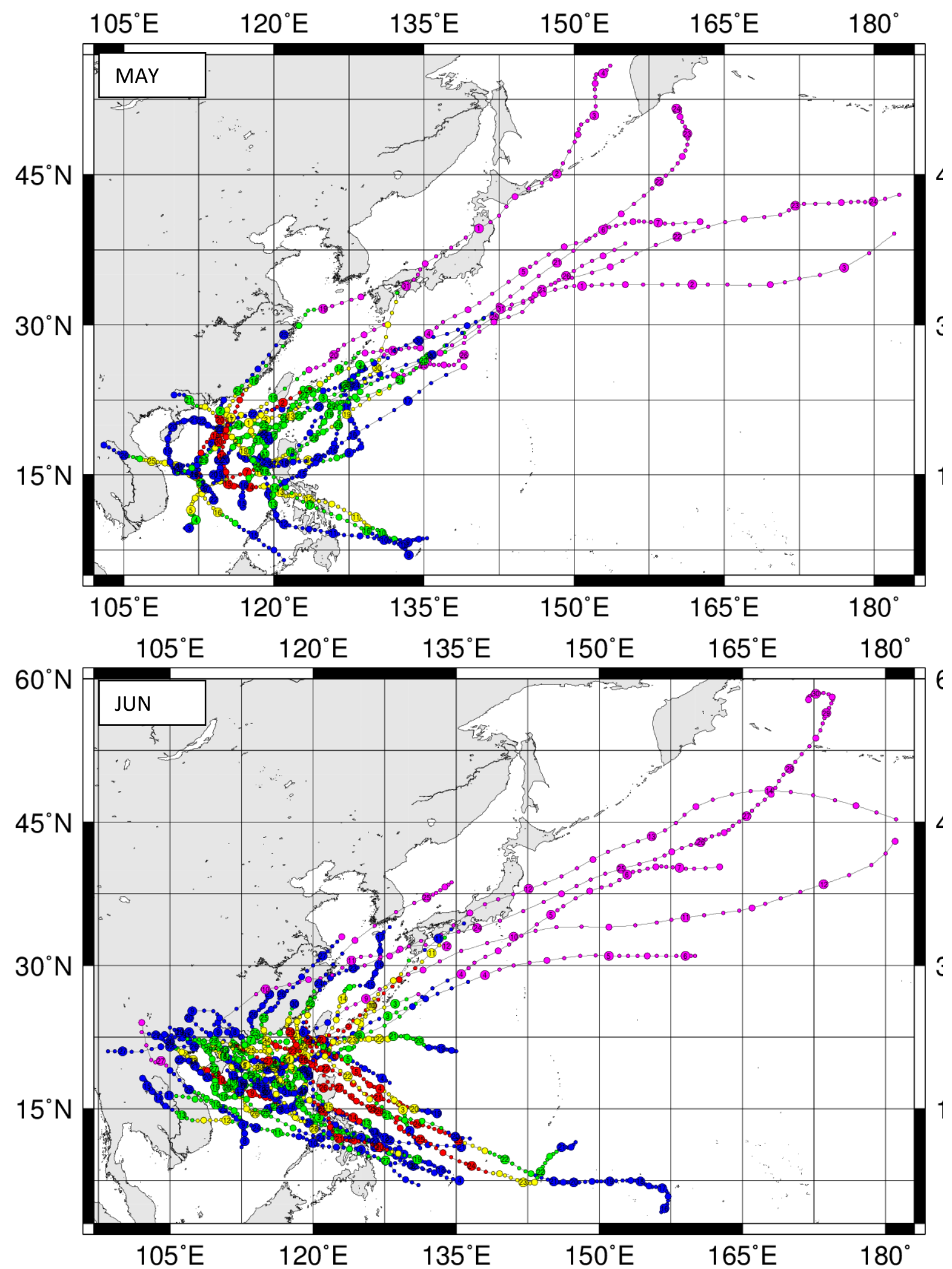

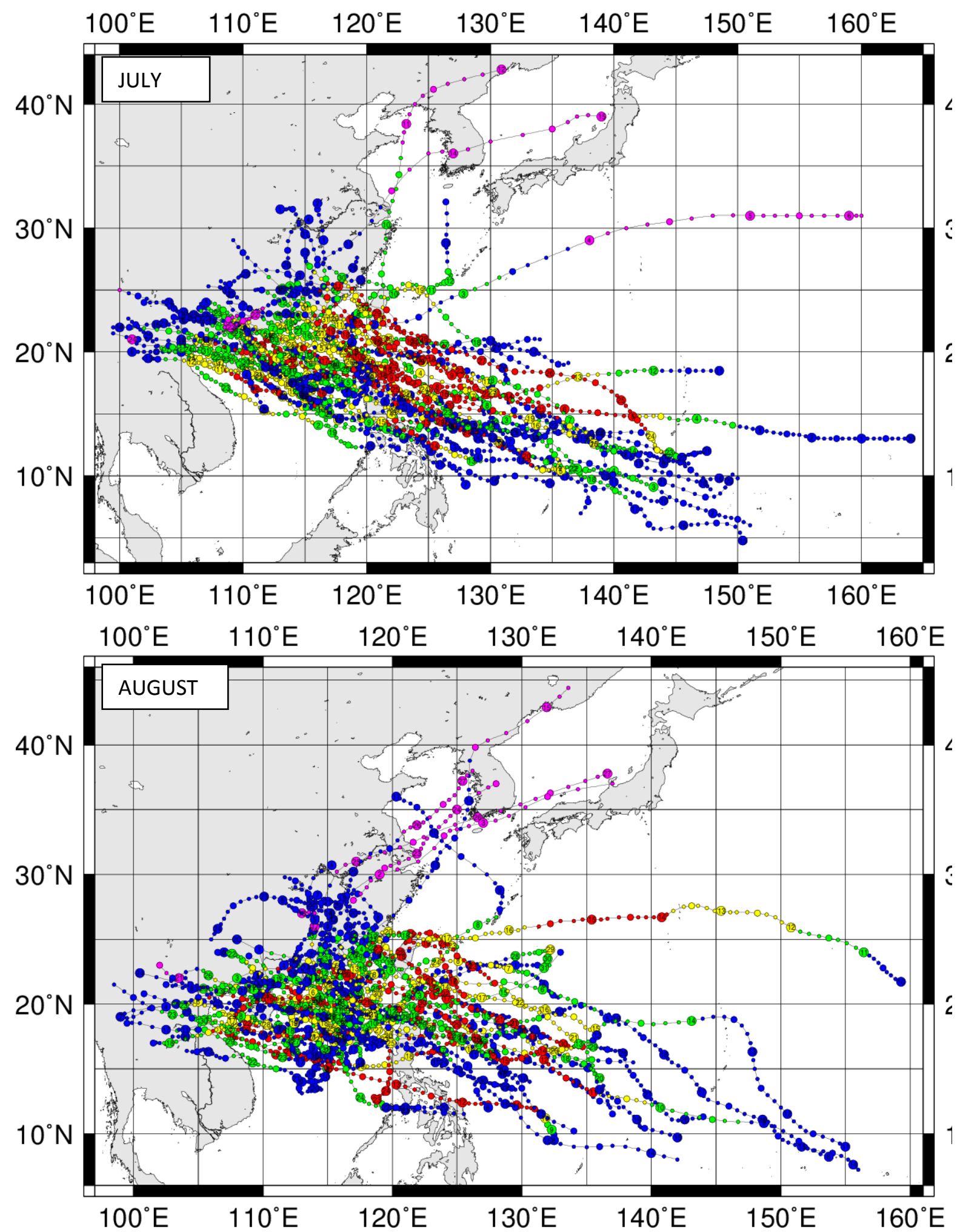

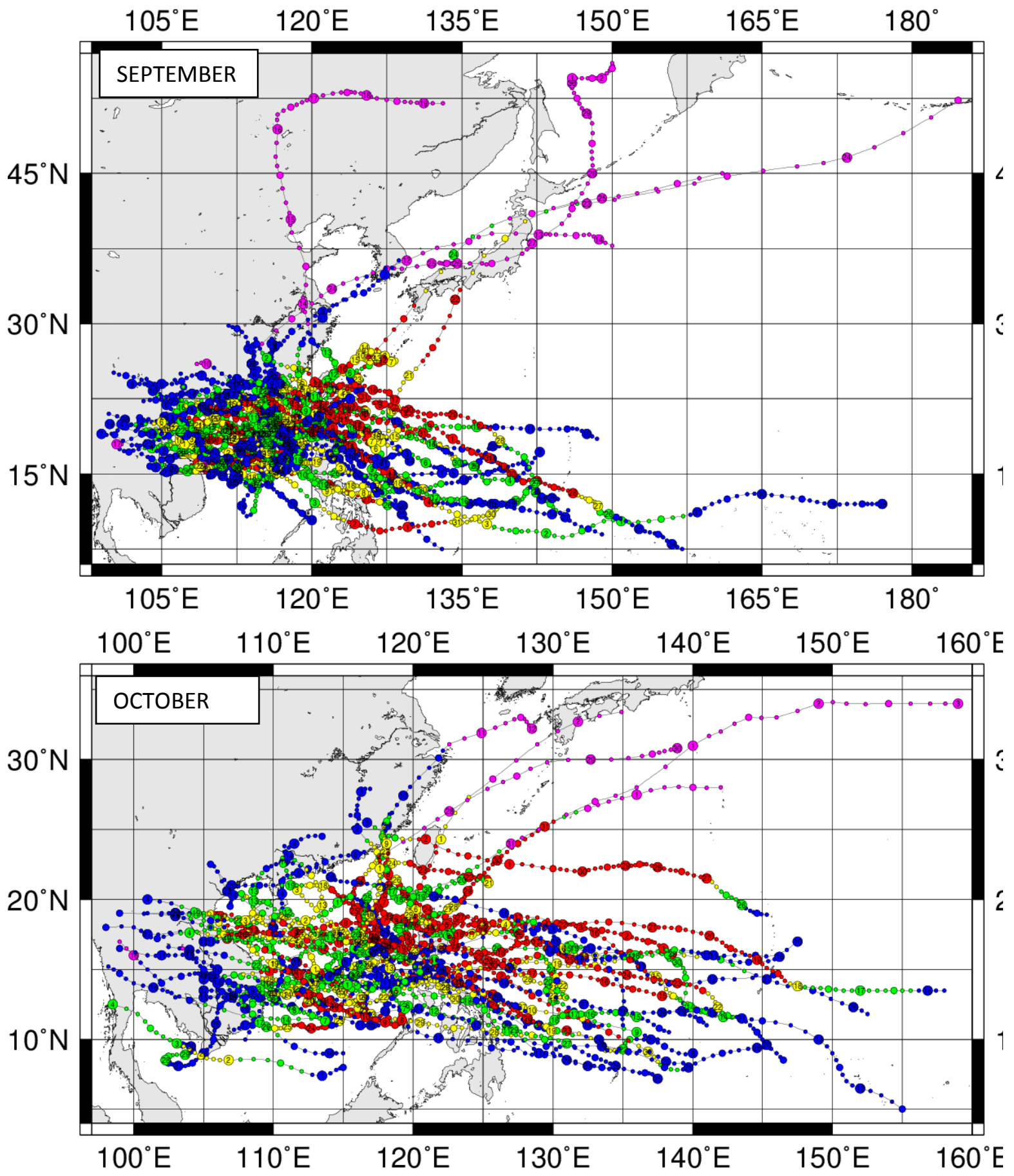

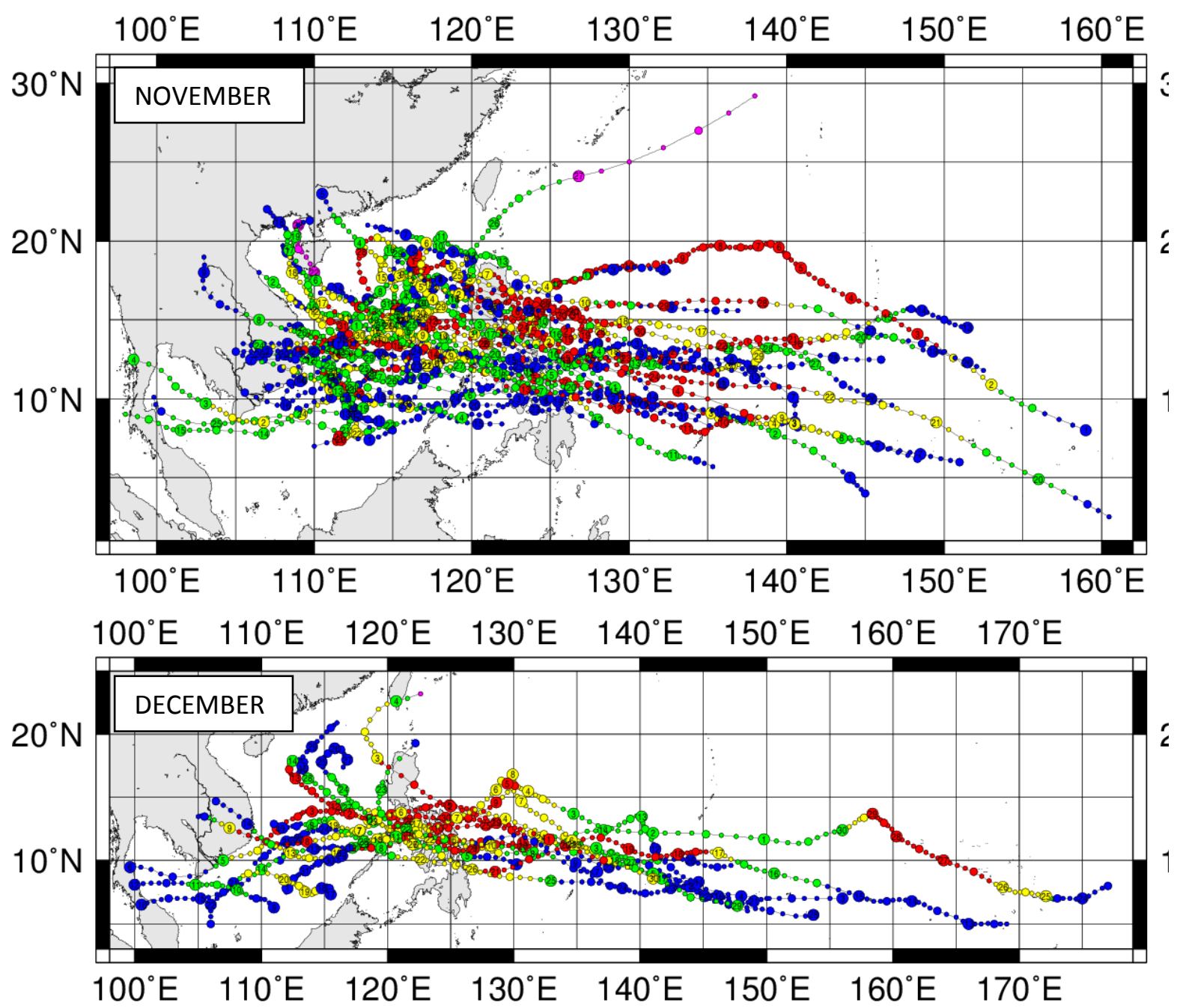

Figure AF3. Tracks of monthly WMO typhoons in 1979-2010. There are 334 typhoons in total, and the typhoon number in each month is assigned in the ().Top to bottom: Mar(4), April(5), May(18), June(32), July(55), August(52), September(60), October(49), November(43), December(16). No typhoon in January and February is recorded. 


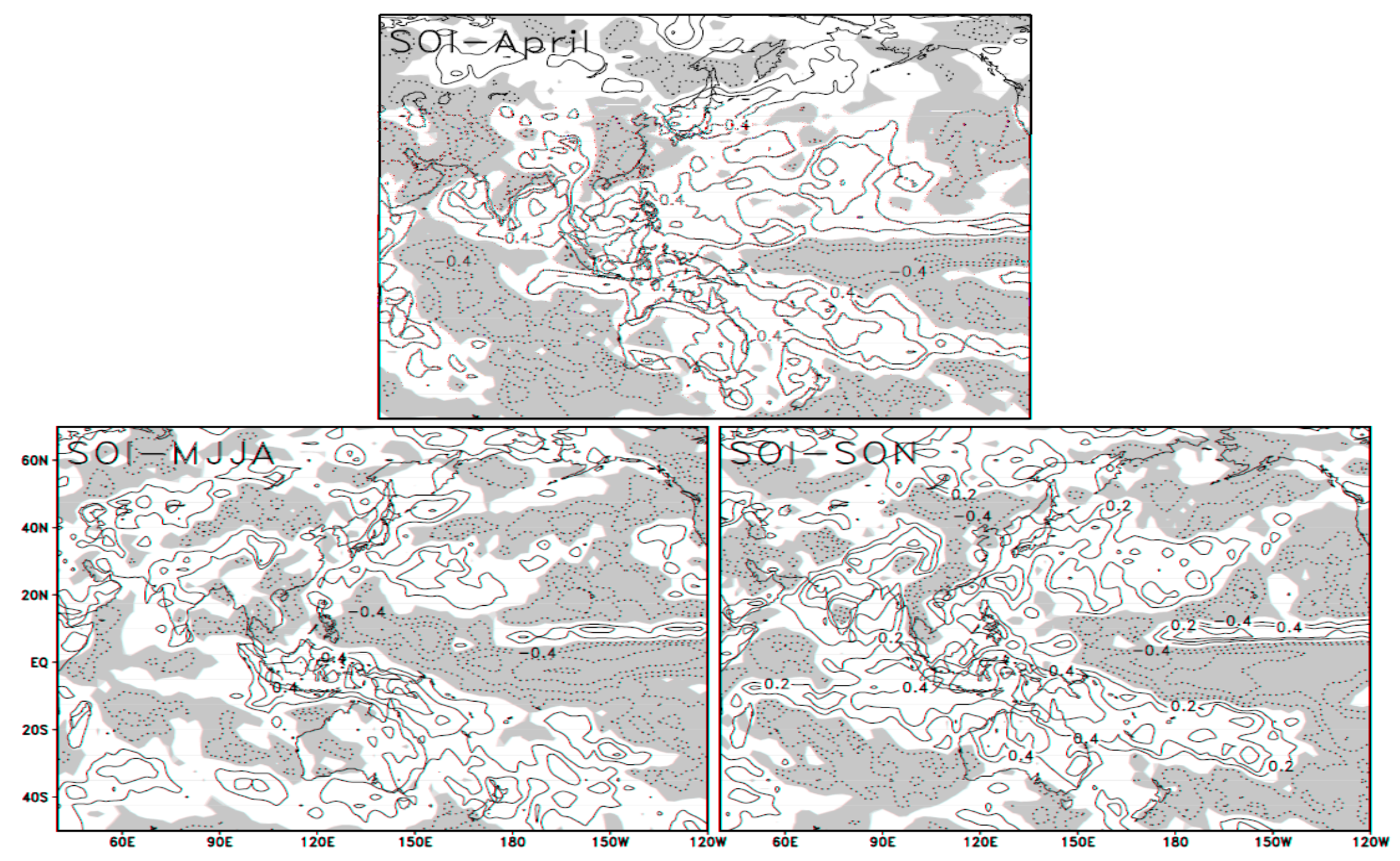

Figure AF4. Correlation map between seasonal JCDAS rainfall and the Southern Oscillation Index (SOI). Only correlation coefficients at 90\% of confidence interval are displayed. 


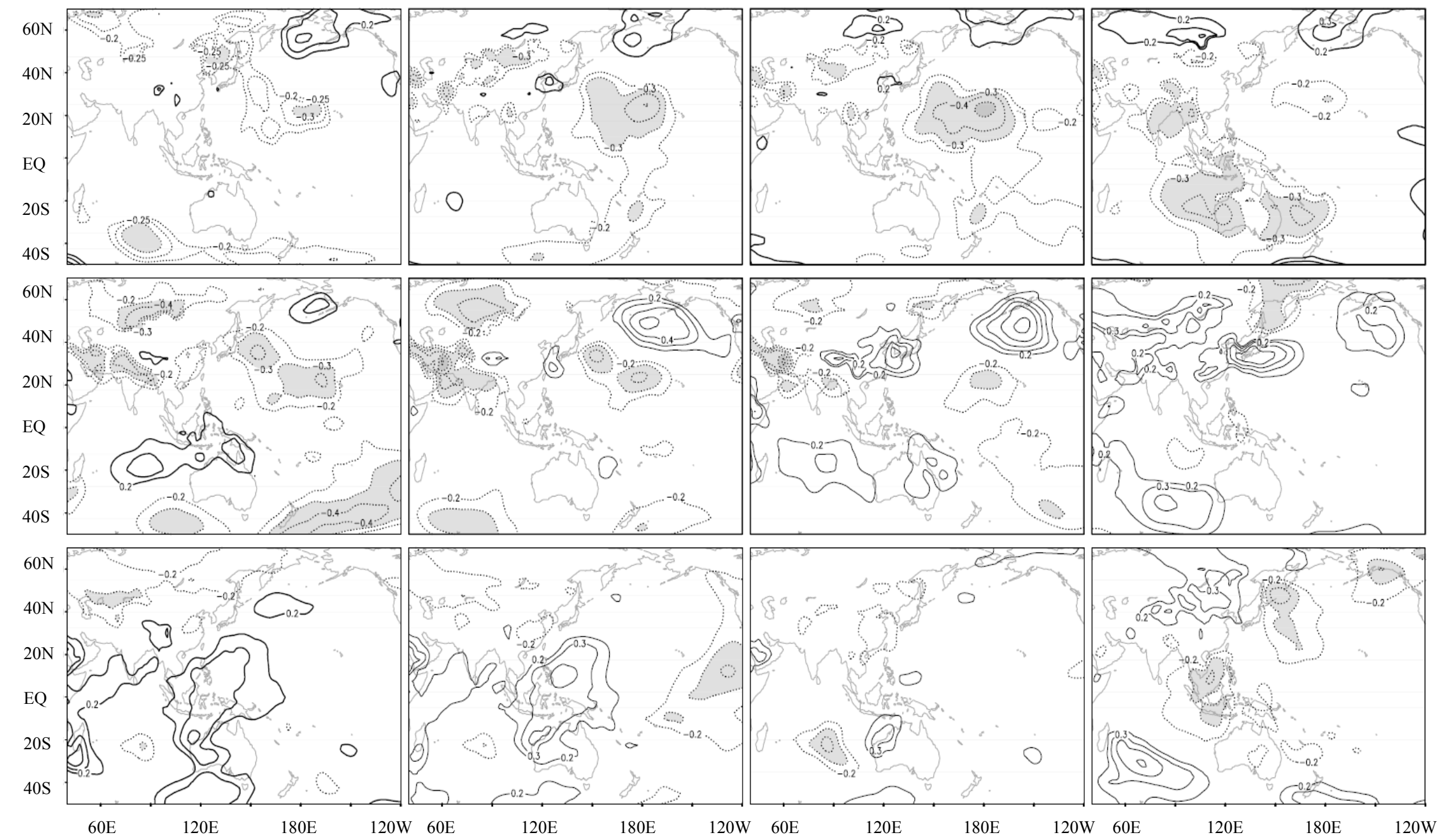

Figure AF5. Correlation maps between MSLP in REG1 domain and Vietnam sub-region rainfall. From left to right: N1,N2,N3,N4 sub-region. From top to bottom: annual, MJJA, DJFM. Only correlation coefficients at 90\% of confidence interval are displayed. Contour level: 0.1 . 

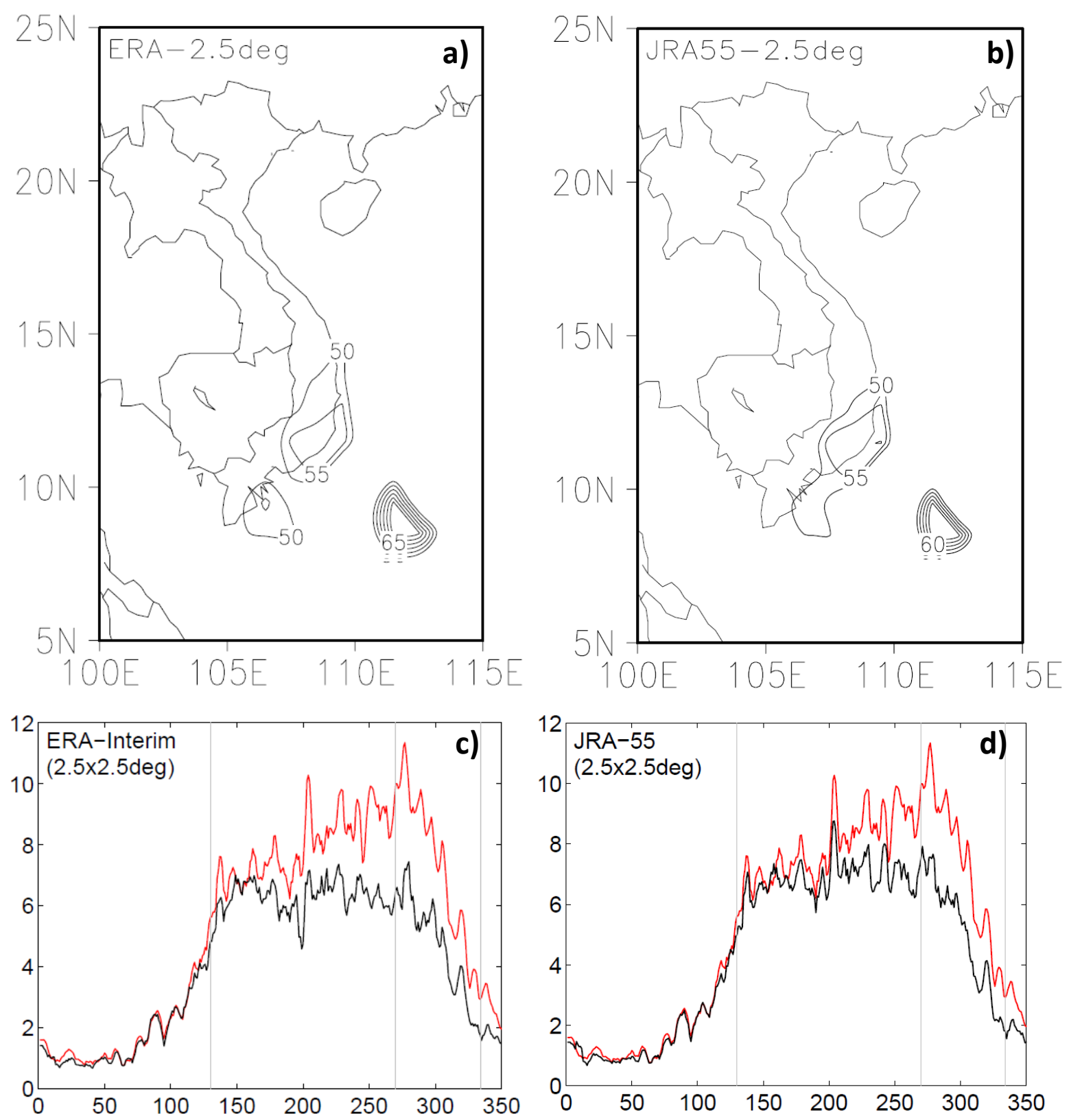

Figure AF6. A sensitivity test on resolution. a-b) Vortex-induced rain days at stations, as a percentage of the total (11680) number of days in the 32 year record (1979-2010). ERA-interim and JRA-55 degraded to $2.5 \times 2.5$ degree latitude/longitude resolution. c-d) Mean temporal distribution of rainfall before (red) and after (black) extracting vortex rainfall in Vietnam (19792010). Red and black line represent for observation and corresponding reanalysis dataset (2.5x2.5degree ERA-interim and JRA-55). Vertical lines from left to right denote for the mean onset (day \#130), withdrawal (day \#264), and 30 November (day \#334). Y-axis: mm/day, X-axis: 365 days. 

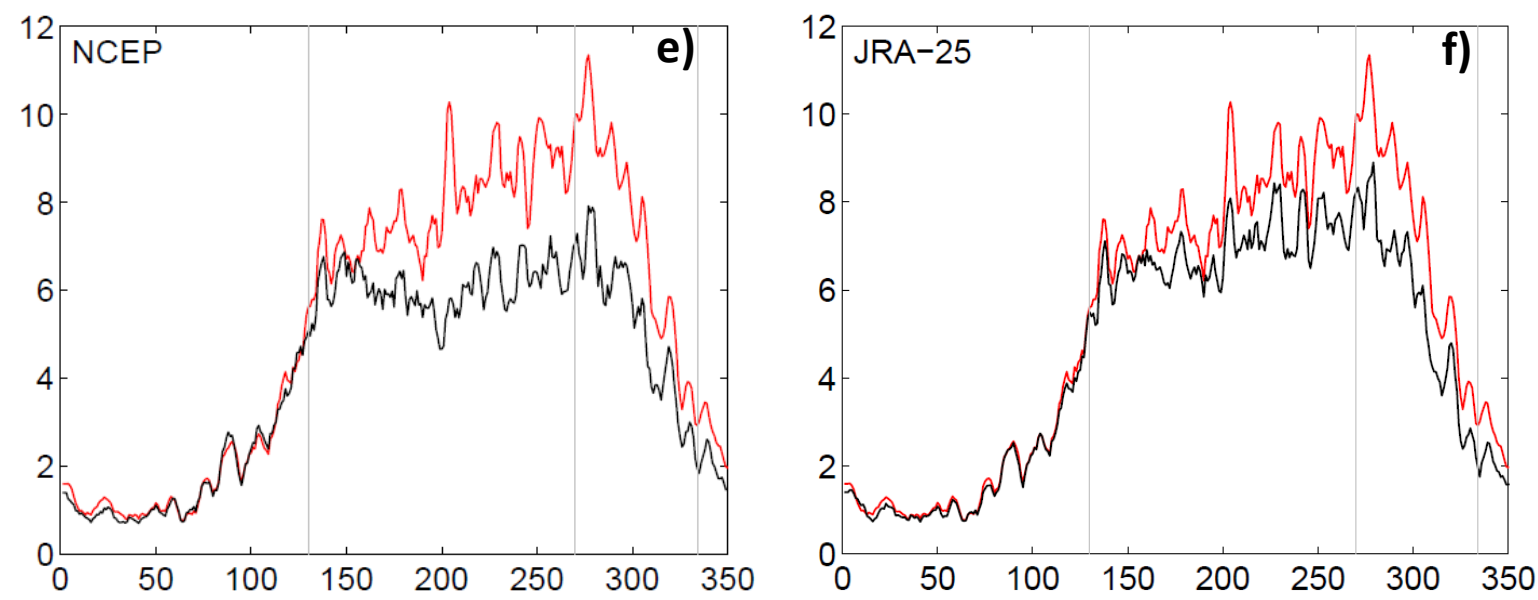

Figure AF6. A sensitivity test on resolution. e-f) Mean temporal distribution of rainfall before (red) and after (black) extracting vortex rainfall in Vietnam (1979-2010). Red and black line represent for observation and corresponding reanalysis dataset (NCEP-DOE (e) and JRA-25 (f) ). Vertical lines from left to right denote for the mean onset (day \#130), withdrawal (day \#264), and 30 November (day \#334). Y-axis: mm/day, X-axis: 365 days. 
a)

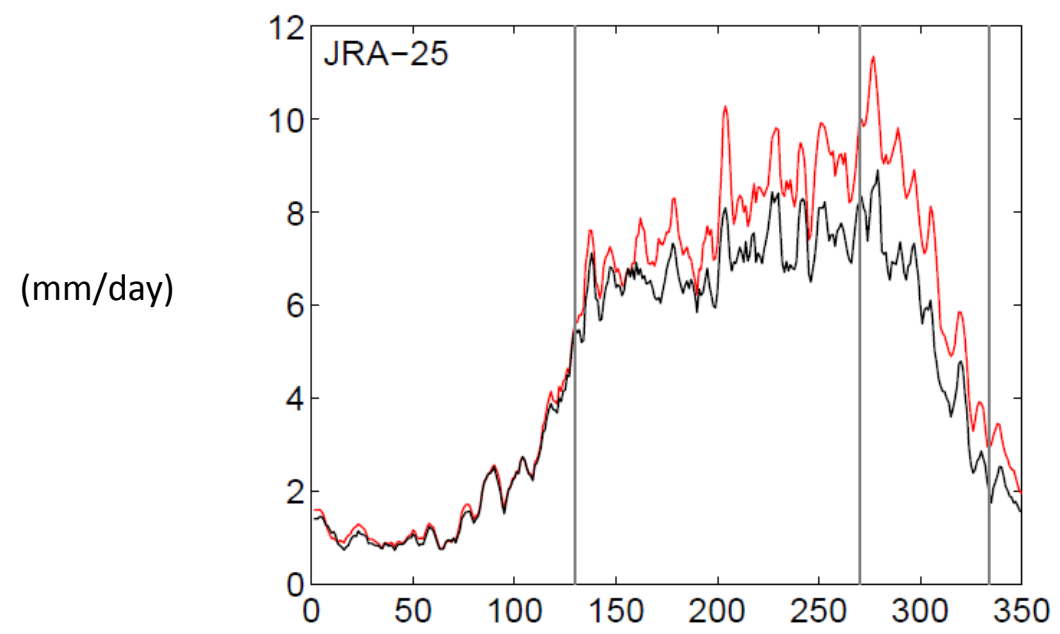

b)

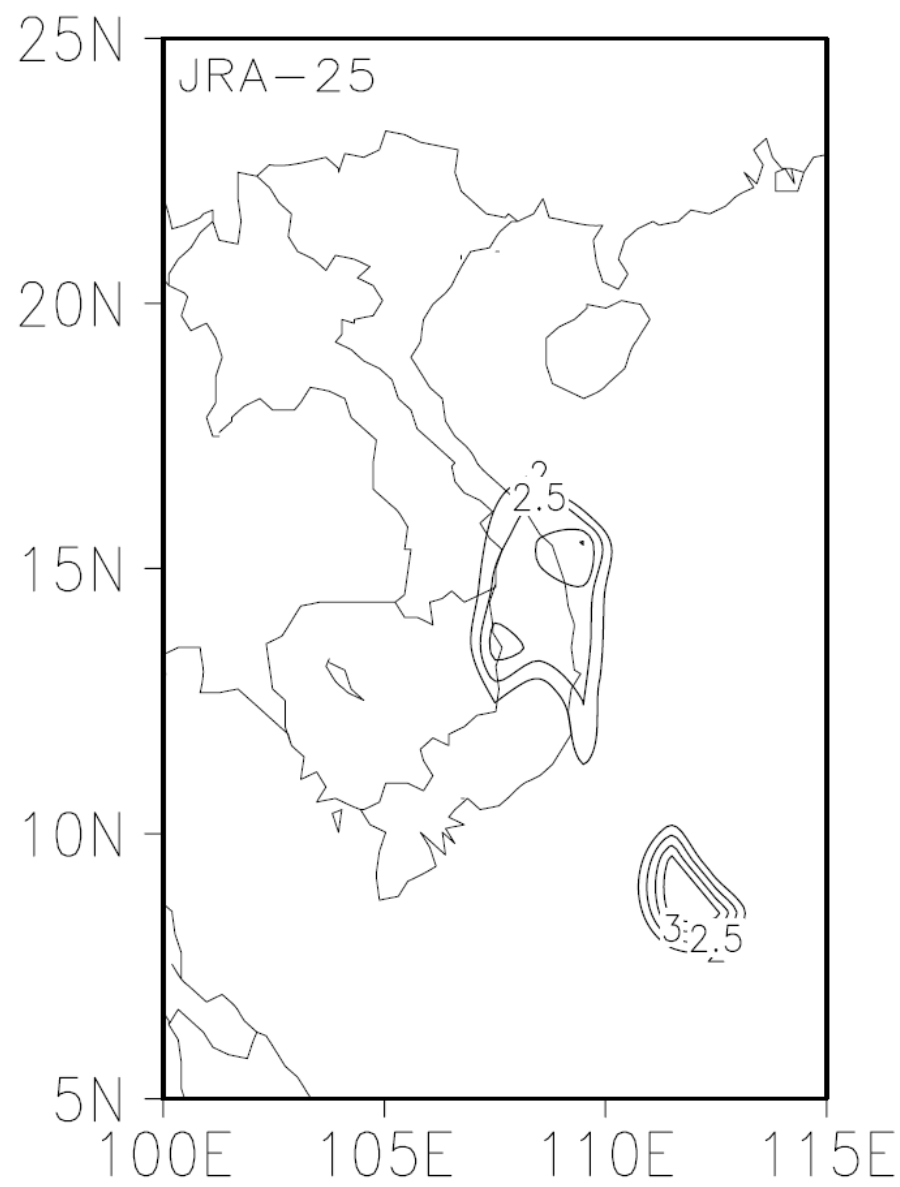

Figure AF7 a) Mean temporal distribution of rainfall before (red) and after (black) extracting vortex rainfall in Vietnam (1979-2010). Red and black line represent for observation and JRA-25 dataset. Vertical lines in a) from left to right denote for mean onset(day \#130), withdrawal(day \#264), 30 November(day \#334). Y-axis: mm/day, X-axis: 365 days. b) Mean spatial distribution of vortex rainfall (difference between observation and reanalysis). Unit: $\mathrm{mm} /$ day. 
c)

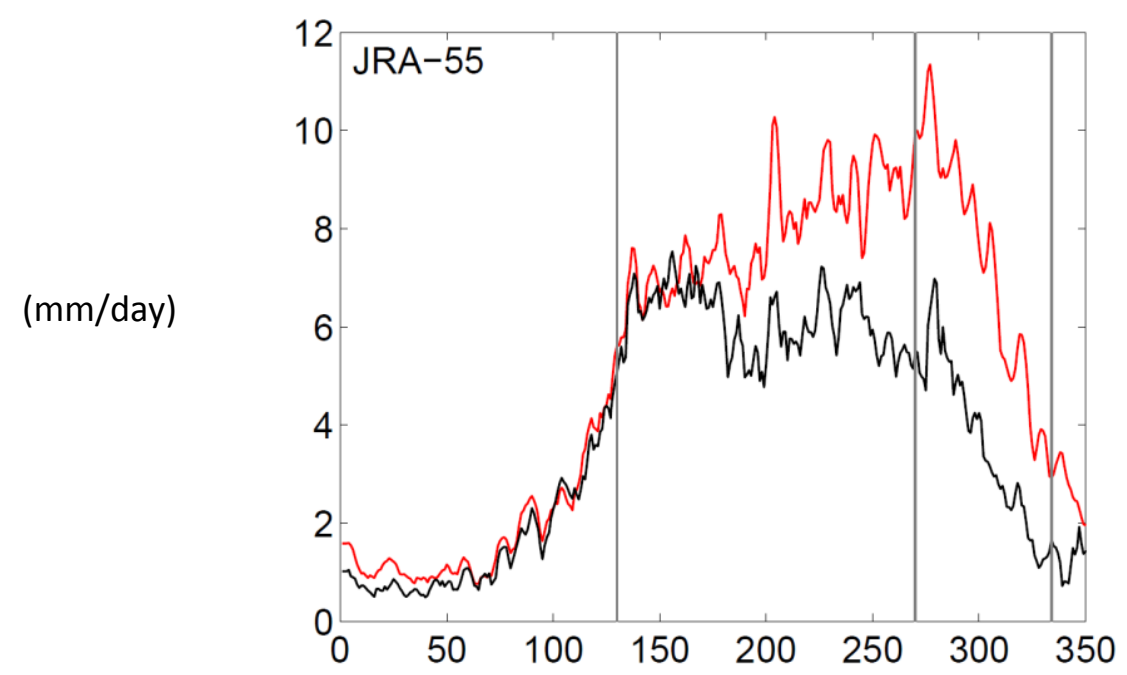

d)

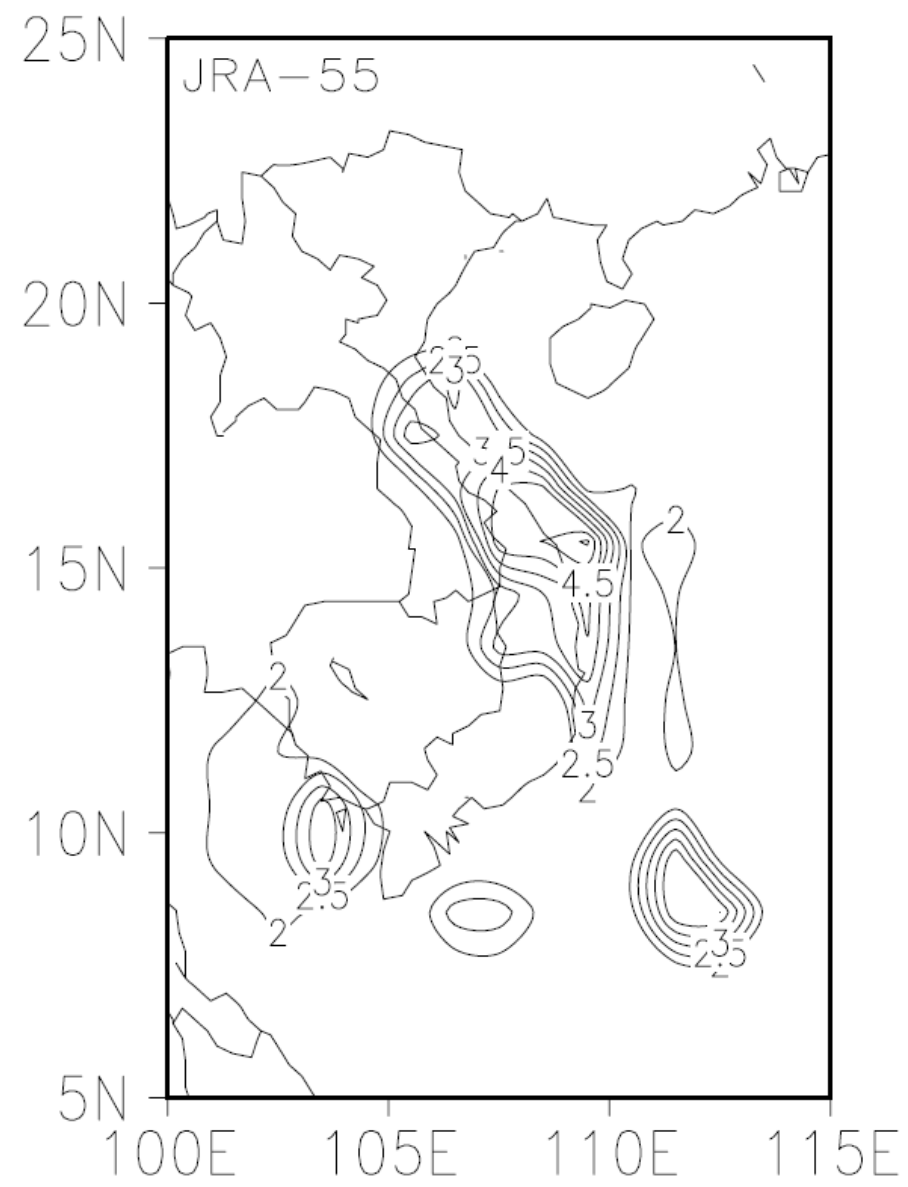

Figure AF7 c-d) Same as Fig. AF7 a-b) except the JRA-55 reanalysis dataset. 
e)

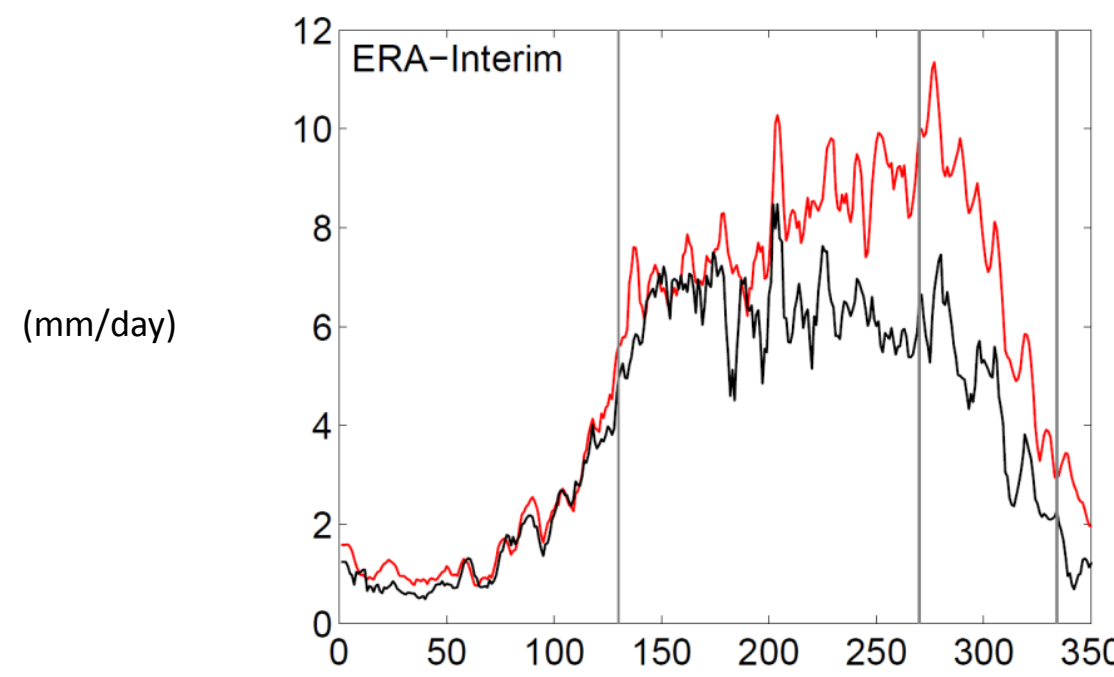

f)

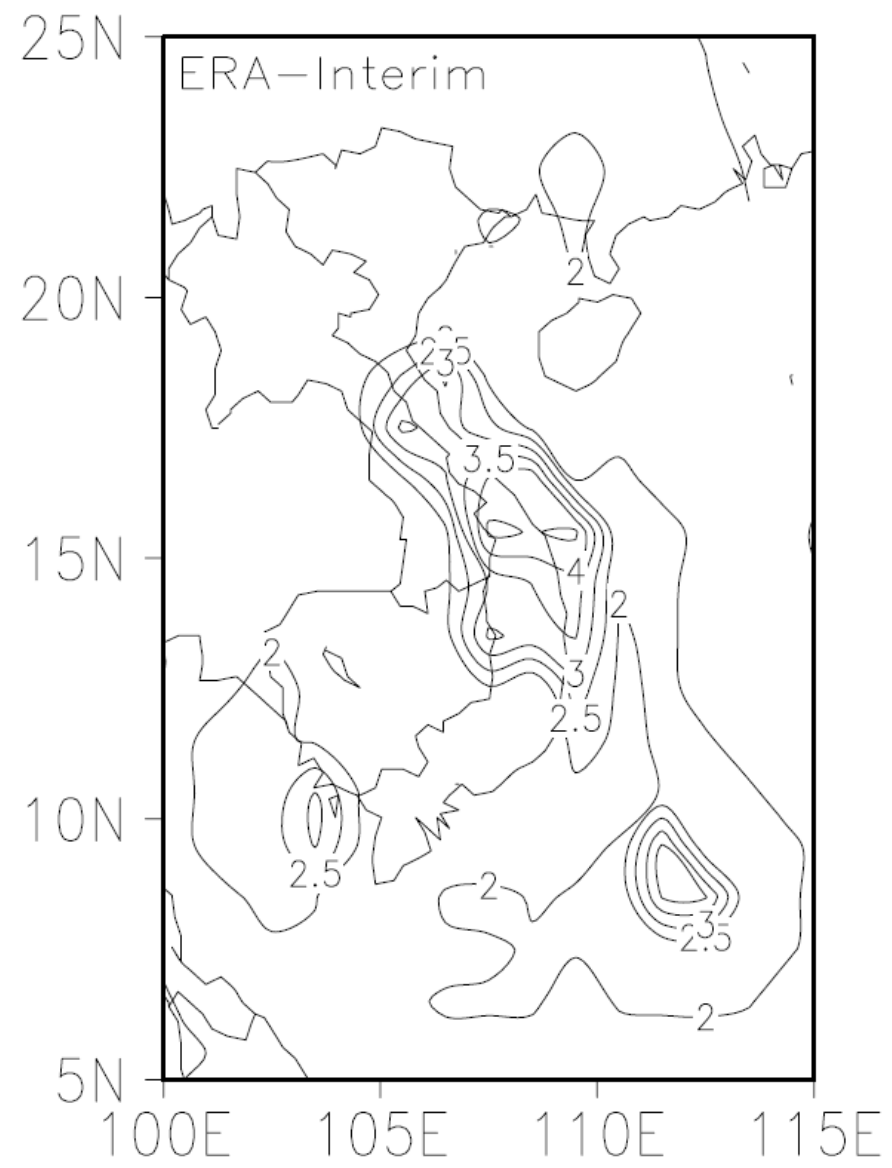

Figure AF7 e-f) Same as Fig. AF7 a-b) excepts the ERA-Interim reanalysis dataset. 


\section{Appendix for methodologies (AM)}

\section{AM.1 Nonparametric Mann-Kendall test}

The description in this section is based on the work of Altava-Ortiz, et al.,2011. The Mann-Kendall (MK) test is based on time data ranking, i.e. each data point is compared with all data points that follow in time, providing the statistic:

$$
S=\sum_{i=1}^{n-1} \sum_{j=i+1}^{n} \operatorname{sgn}\left(x_{j}-x_{i}\right)
$$

Where $\mathrm{x}_{\mathrm{i}}$ is the data value at time $\mathrm{I}, \mathrm{n}$ is the length of the data set and $\operatorname{sgn}(\mathrm{z})$ is equal to +1 , $0,-1$ if $\mathrm{z}$ is greater than, equal to, or less than zero, respectively. The null hypothesis is that the data are independently and identically randomly distributed, and no trend existed in the dataset. For the MK statistic holds:

$$
\begin{gathered}
\mathrm{E}(\mathrm{S})=0 \\
\operatorname{Var}(S)=\frac{1}{18}\left(n(n-1)(2 n+5)-\sum_{i=1}^{n} t_{i}(i-1)(2 i+5)\right.
\end{gathered}
$$

where $t_{i}$ denotes the number of tied values of extent $i$.

For $\mathrm{n}>10$, the test statistic $\mathrm{Z}_{\mathrm{MK}}$ is:

$$
Z_{M K}= \begin{cases}\frac{\mathrm{S}-1}{\sqrt{\operatorname{Var}(\mathrm{S})}} & \text { for } \mathrm{S}>0 \\ 0 & \text { for } \mathrm{S}=0 \\ \frac{\mathrm{S}+1}{\sqrt{\operatorname{Var}(\mathrm{S})}} & \text { for } \mathrm{S}<0\end{cases}
$$

$\mathrm{Z}_{\mathrm{MK}}$ approximately follows a standard normal distribution (Kendall, 1962). In the case of two sided test, if $\left|Z_{\mathrm{MK}}\right|>1.96$ then the existing trend is considered to be statistically significant at the significance level of 0.05 .

\section{AM.2 Sen's slope method}

The idea of Sen (1968) is a simple and robust estimator of $\beta$ based on Kendall's rank correlation tau for points as well intervals. The point estimator is the median of the set of slopes $\left(Y_{j}-Y_{i}\right) /\left(t_{j}-t_{i}\right)$ joining pairs of points with $t_{i} \neq t_{j}$, and is unbiased. Sen's method 
proceeds by calculating the slope as a change in observation per change in time, as shown in Equation (A2.4):

$$
\mathrm{X}_{i j}=\frac{Y_{j}-Y_{i}}{t_{j}-t_{i}} \quad(1 \leq \mathrm{i}<\mathrm{j} \leq \mathrm{n})
$$

where $\mathrm{X}_{\mathrm{ij}}$ is slope of the lines connecting each pair of point $\left(\mathrm{t}_{\mathrm{i}}, \mathrm{Y}_{\mathrm{i}}\right)$ and $\left(\mathrm{t}_{\mathrm{j}}, \mathrm{Y}_{\mathrm{j}}\right) \forall\left(\mathrm{t}_{\mathrm{i}} \neq \mathrm{t}_{\mathrm{j}}\right)$ between data point $Y_{j}$ and $Y_{i} . Y_{j}, Y_{i}$ is observations at time $t_{j}$ and $t_{i}$, respectively.

If multiple observations are collected at a given time, two options exist. The first option is to simply combine the observations for a given time step into a single observation of central tendency (e.g. mean, median) and proceed as (A2.1). The second option is to calculate a slope for each individual observation. Then Sens's estimator slope is given by the median slope:

$$
\text { Sen's slope }=\beta^{*}=\left\{\begin{array}{lr}
X_{(M+1) / 2}, & N=2 M+1 \\
\left(X_{M}+X_{(M+1)}\right) / 2, & N=2 M
\end{array}\right.
$$

where $\mathrm{N}$ is number of calculated slopes.

Thus, $\beta^{*}$ is the median of the $N$ numbers. Sens's method also allows determination of whether the median slope is statistically different from zero. A confidence interval is developed by estimating the rank for the upper and lower confidence interval and using the slopes corresponding to these ranks to define the actual confidence interval for $\beta^{*}$. For a two-sided confidence interval about the median slop, first find the $\mathrm{Z}_{\text {statistic }}$ for a two-tailed normal distribution test. For example, if a two-sided confidence interval of $95 \%$ is desired, find $\mathrm{Z}_{(1-0.05 / 2)}=\mathrm{Z}_{0.975}=1.96$. Next, estimate the variance of the MK statistic. $\operatorname{Var}(\mathrm{S})$ is calculated by equation (A2.2).

To estimate the range of ranks for the specified confidence interval, find $\mathrm{C}$ using:

$$
C_{\alpha}=Z_{1-\alpha / 2} * \sqrt{\operatorname{VAR}(S)}
$$

The ranks of the lower (L) and the upper (U) confidence are identified:

$$
\begin{aligned}
& \mathrm{L}=\left(\mathrm{k}-\mathrm{C}_{\alpha}\right) / 2 \\
& \mathrm{U}=1+\left(\mathrm{k}+\mathrm{C}_{\alpha}\right) / 2
\end{aligned}
$$


Finally, choose the slopes corresponding to $\mathrm{L}$ and $\mathrm{U}$. Note that the median slope is then defined as statistically different from zero if the zero does not lie between the upper and lower confidence limits.

\section{AM.3 The paired t-test}

A paired t-test is used to compare whether two groups have different average values. In this Ph.D work, the paired t-test is used to check the rainfall and temperature anomalies between the Truong-Sa station and all other climate sub-regions (Section 4.1).

This difference is to be reliable if

1) the difference between the averages of two sets of data is large,

2) the sample size is large, and

3) responses are consistently close to the average values and not widely spread out (the standard deviation is low).

Before performing the test a threshold value is chosen, called the significance level of the test, traditionally $0.05,0.01$ or 0.001 and denoted as $\alpha$. The p-value obtained in Section 4.1 is smaller than the $\alpha=0.01$; it suggests that the climate in Truong-Sa station (rainfall and temperature anomalies) is significantly different to other sub-regions.

\section{AM.4 Uncertainty in trend detection in observation data}

Identifying the trends is always a hard job. Two factors, the quality of observation data (outliers) and the chosen period of time, will be giving to show how they are greatly affected to the trends.

Outliers: Just by changing the maximum/minimum value (marked by a dotted-red circle in diagrams below) in a timeserie, its trend is conversed from increase to decrease. 

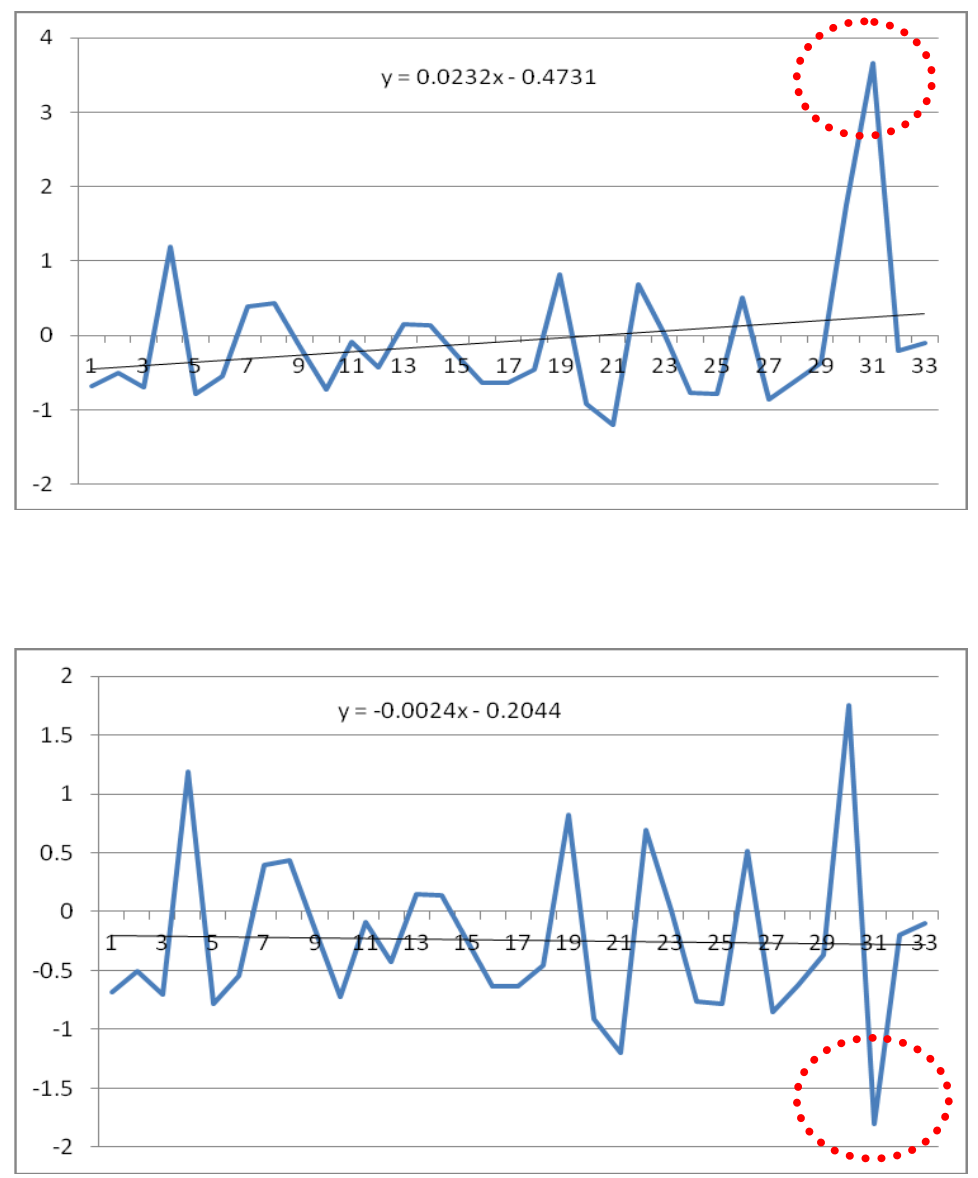

Sensitivity with the selection of period of time:

The first diagram below is shown the variation of monthly temperature anomaly in a station in Vietnam from January 1971 to September 2014. The trend of change during this whole period is increase. However, if we select a 10 -year time, marked by a dotted-red circle, the trend of change in this 10-year period is decrease as shown in the second diagram. 

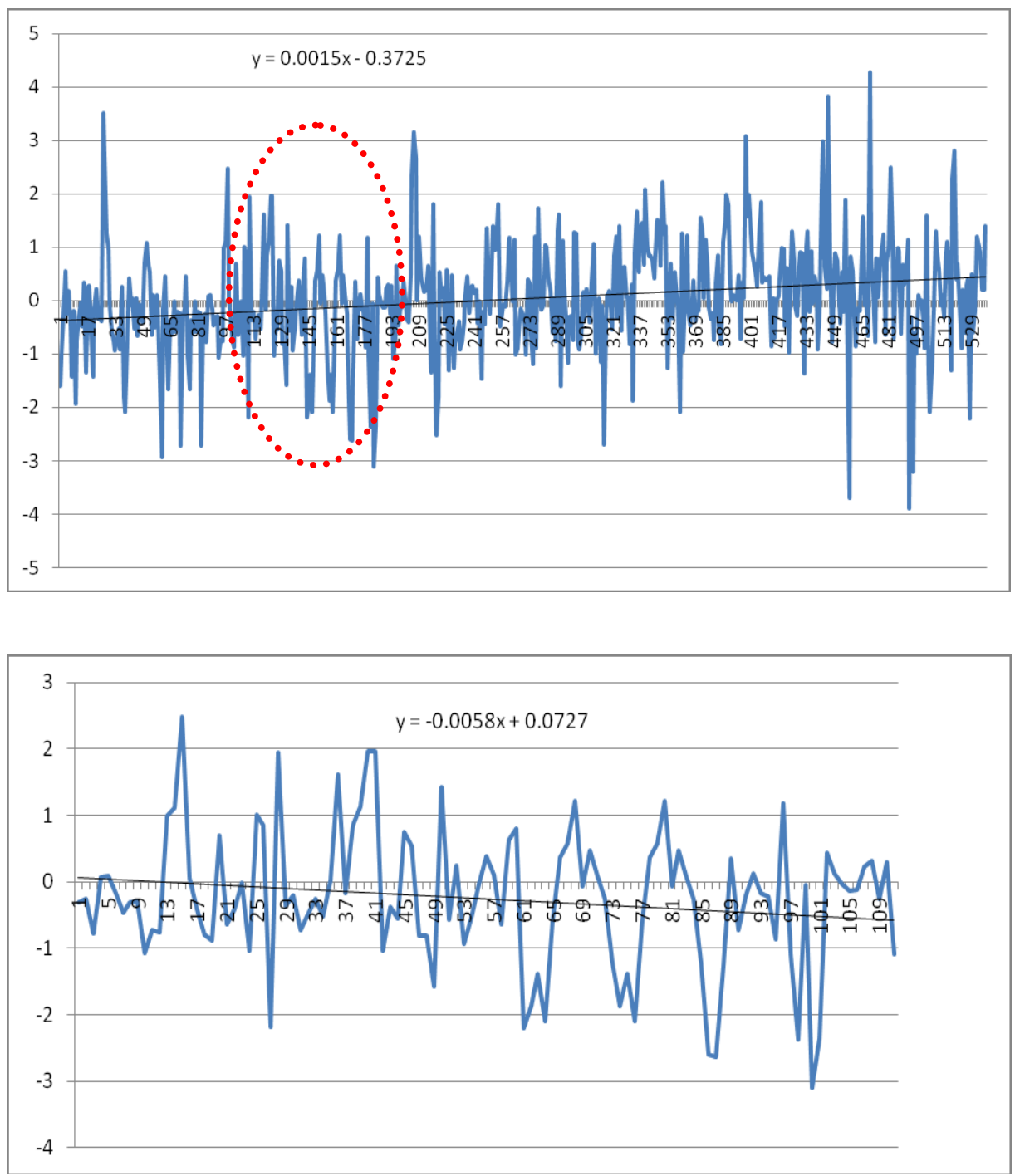

Therefore, the outliers and the selected period of time are sensitivity in trend detection in observation data. 


\section{Appendix for programming codes (AC)}

\section{AC.1 Data access and retrieve (download_JDAS.sh)}

$\# ! /$ bin/sh

\#\#\#\#\# SETUP HTTP PROXY

export http_proxy=http://www-cache.vuw.ac.nz:8080/

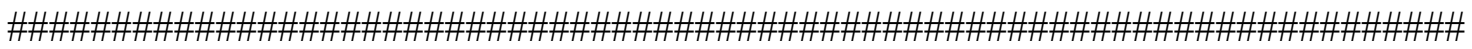

\# initialization

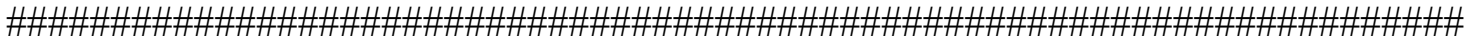

set -eu

: $\$\{$ user:=XXXXXXXX $\}$

: \$\{pass:=XXXXXXXX\}

: \$\{option:="-N -r -c -np -w 180 --level=0" \}

: \$\{URL:=http://ds.data.jma.go.jp/gmd/jra/download/data/ElmntFinal $\}$

: $\$\{$ prefix: $=$ cpratsfc $\}$

: $\$\{$ suffix:=idx $\}$

: $\$\{$ start: $=200008\}$

: $\$\{$ end: $=200012\}$

: $\$\{$ WGET:=/usr/bin/wget $\}$

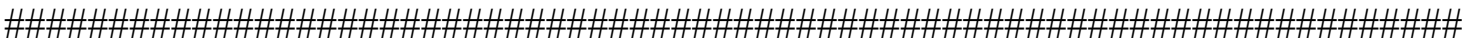

\# getting JRA-25 data

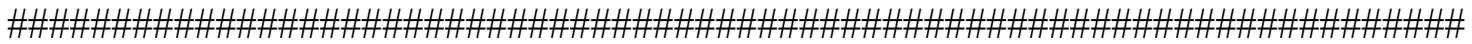

year=’echo $\$\{$ start $\} \mid$ cut -c 1-4'

month='echo $\$\{$ start $\} \mid$ cut -c 5-6'

while $[\$\{$ year $\}$ \{month $\}$-le $\$\{$ end $\}]$

do

$\$\{$ WGET $\} \backslash$

$\$\{$ option $\} \backslash$

$\$\{$ user:+--http-user $=\$\{$ user $\}\} \backslash$

$\$\{$ pass:---http-passwd $=\$\{$ pass $\}\} \backslash$

\$ $\{$ URL $\} /$ \{ prefix $\} /$ \{prefix $\} " . p h y 2 m 6 h " \$\{$ year $\}$ \{month $\}$ 
if [ $\$$ month $\}$-eq 12$]$; then

month $=01$

year='expr $\$\{$ year $\}+1$ '

else

month='echo $\$\{$ month $\}$ awk ' printf("\%02d",\$1+1)\}"

fi

done

exit 0

\section{AC.2 Climate trend detection (trend.m)}

\%Burkey J. 2011. Seasonal Kendall Test with Slope for Serial Dependent

\%Data, http://www.mathworks.com/matlabcentral/fileexchanges/22389-seasonal-kendall

\%The Mann-Kendall Tau-b non-parametric function computes a coefficient representing strength and direction of a trend

$\%$ for equally spaced data. ...Example syntax to use the function is:

$\%[$ taub tau h sig Z S sigma sen $n$ senplot cilower ciupper] = ktaub(datain, alpha,wantplot) $\% \% \%$

\%\%\% Quang.Nguyen@vuw.ac.nz edited in June 2012 in order to testing the rainfall trends, compared to least squared estimations.

load('trend.mat');

missinglowess $=0$;

\%1) Trend monsoon rainfall in 60Stations

data1 $=[\operatorname{trend} . \operatorname{data}(:, 1: 2)]$;

data1 $($ any $($ isnan $($ data 1$), 2),:)=[]$;

[taub , , $\sim, \operatorname{sig} \mathrm{Z}, \sim$, sigma sen $, \sim, \sim$, Cllower Clupper $, \sim, \sim, \sim$, nsigma] $=\operatorname{ktaub}($ data1, $.05,1)$;

tolerance $=($ Clupper - Cllower $) / 2$;

results $=[$ taub sigma CIlower CIupper Z sig sen tolerance $]$; 


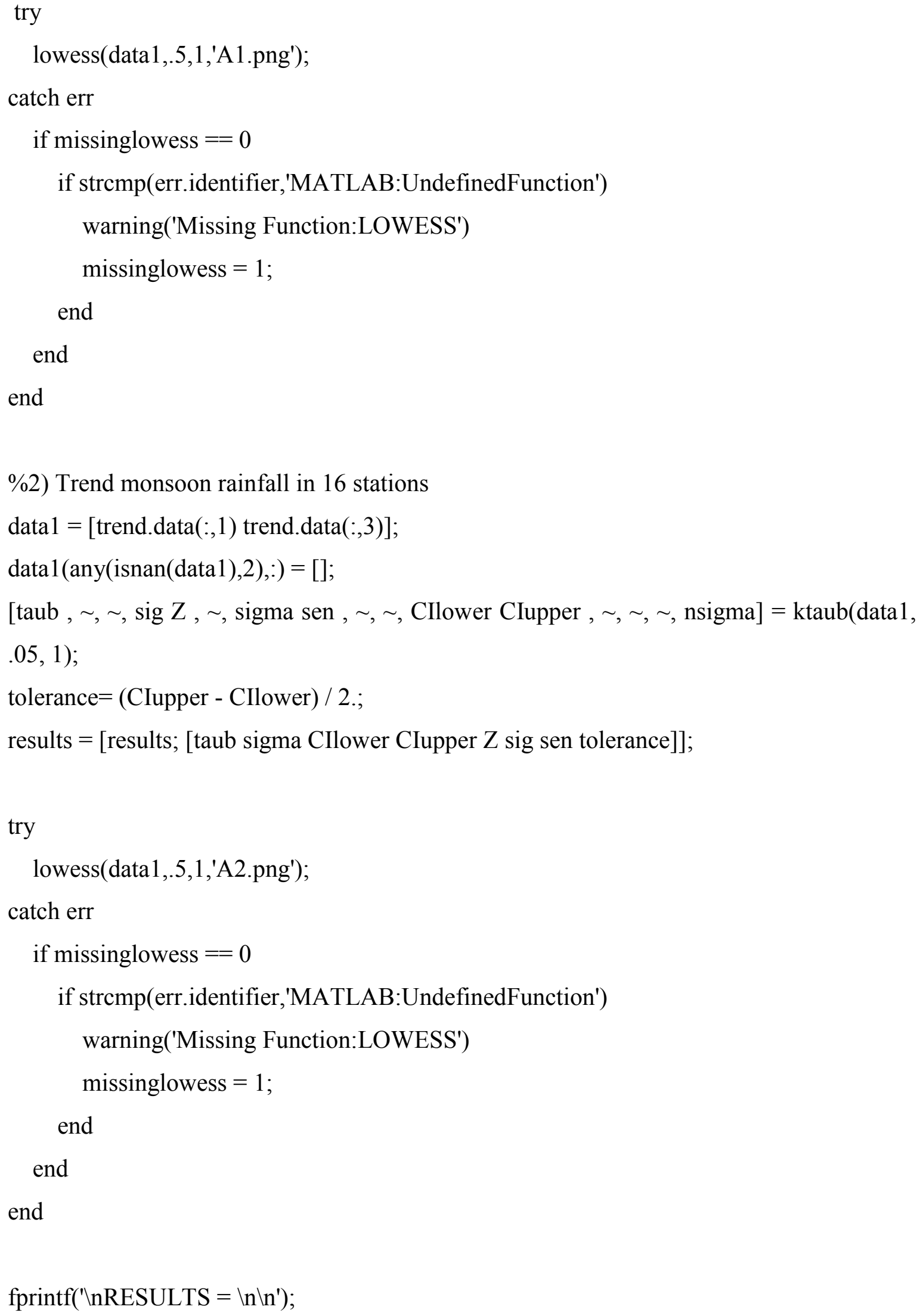


fprintf(' tau-b sigma Cllower Clupper $\quad \mathrm{Z}$ sig sen $\quad$ Tolerance $\backslash \mathrm{n}$ ')

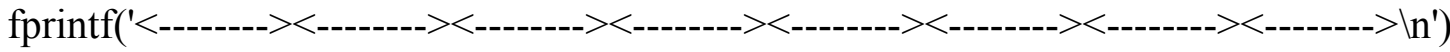

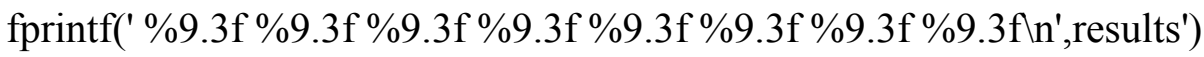

fprintf('ınWriting data to excel spreadsheet TCrain_trend.xls $\backslash n$ ');

\section{AC.3 Plotting the synoptic weather pattern (synop_pattern.gs)}

function synop_pattern(args)

if $(\operatorname{args}=")$

help()

return

endif

'reinit'

'open ugrdprs0925.anlp6h.ctl'

'open ../vgrdprs0925/vgrdprs0925.anlp6h.ctl'

'open ../cpratsfc/cpratsfc.phy2m6h.ctl'

'open ../lpratsfc/lpratsfc.phy2m6h.ctl'

'open ../prmslmsl/prmslmsl.anlp6h.ctl'

*Domain Southeast Asian Sea -Vietnam (10S-40N; 80-160E)

$\mathrm{x} 1=33$

$\mathrm{x} 2=65$

$\mathrm{y} 1=33$

$\mathrm{y} 2=53$

$\operatorname{doit}(\mathrm{x} 1, \mathrm{x} 2, \mathrm{y} 1, \mathrm{y} 2, \operatorname{args})$

$* * * * * * * * * * *$ END OF MAIN FUNCTION

function doit(x1,x2,y1,y2,args)

'set $t$ 'time(args)

'set display color white' 
'c'

'set map 15'

'set grads off'

'set y 'y1' 'y2

'set $\mathrm{x}$ 'x1' 'x2

*RAINFALL

'set gxout shaded'

'color 2015020 -kind white->red'

'd (cpratsfc.3 + lpratsfc.4)'

'cbar'

*STREAMLINES

'set gxout stream'

'set ccolor 15 '

'set strmden 5'

'd ugrdprs. 1;vgrdprs. 2'

*MSLP:

'set gxout contour'

'set cint 5'

'set ccolor 1'

'd prmslmsl.5/100'

* DRAW TITLE:

'q time'

tmp1 = sublin(result, 1$)$

$\mathrm{t} 1=\operatorname{subwrd}(\operatorname{tmp} 1,3)$

'draw title 925hPa pattern: 't1

* PRINTING: 
delta $=\mathrm{x} 2-\mathrm{x} 1$

if $($ delta $<40)$

'printim Smalldomain't1'.png'

else

'printim Bigdomain't1'.png'

endif

'q 112xy 107.6 16.4'

Hue_x=subwrd(result, 1 )

Hue_y=subwrd(result,2)

'draw wxsym 25 'Hue_x' 'Hue_y' 0.515 '

function time(yyyymmdd)

YY=substr(yyyymmdd,1,4)

$\mathrm{MM}=$ substr(yyyymmdd,5,2)

$\mathrm{DD}=\operatorname{substr}($ yyyymmdd, 7,2)

Delta $=1460$

incr $=$ YY -1979

if $(\mathrm{MM}=09)$; $\mathrm{sSep}=973$; endif

if $(\mathrm{MM}=10)$; $\mathrm{sOct}=1096$; endif

if $(\mathrm{MM}=11) ; \mathrm{sNov}=1220$; endif

if $(\mathrm{MM}=12) ; \mathrm{sDec}=1340$; endif

if $(\mathrm{MM}=09)$

timeslice $=(\mathrm{sSep}+$ Delta $*$ incr + DD*4 $)$

endif

if $(\mathrm{MM}=10)$

timeslice $=\left(\mathrm{sOct}+\right.$ Delta $*$ incr $\left.+\mathrm{DD}^{*} 4\right)$

endif 
if $(\mathrm{MM}=11)$

timeslice $=(\mathrm{sNov}+$ Delta*incr $+\mathrm{DD} * 4)$

endif

if $(\mathrm{MM}=12)$

timeslice $=(\mathrm{sDec}+$ Delta $*$ incr $+\mathrm{DD} * 4)$

endif

say 'Time_slice for this SYNOP pattern:'

say timeslice

return(timeslice)

*

* help

*

function help()

say' '

say ' Usage:'

say ' synop_pattern YYYYMMDD'

say"

say ' Example: synop_pattern 19800926'

return 


\section{VITA}

Nguyen Dang Quang was born on $25^{\text {th }}$ April 1978 in Hai-Phong, Vietnam. After completing his secondary education at Ngo-Quyen school in Hai-Phong, he passed a national exam to enroll in the Vietnam National University, Hanoi (VNU).

In 2000 he received the Bachelor's degree in Meteorology and Climatology. In four following years, he worked in the Department of Meteorology, VNU as a research assistant in the climate modeling group. This job offered him the first overseas trips to the International Centre for Theoretical Physics in Trieste as a young collaborator of the Earth System Physics Section. By the same time, he started to register his name in a master course in meteorology in VNU.

Graduated the master's degree in 2004, he went to the National Centre for Meteorological Research, Metéo-France to pursuit training courses in the Climate and large scale modeling Group and the Modelling and Data assimilation Group.

In 2006 he came back Vietnam, joined and became a weather forecaster in the National Centre for Hydrometeorolgocial Forecastings (NCHMF). This job gave him opportunities to be the NCHMF's representative in the Asia-Pacific Economic Cooperation (APEC) Climate Center.

He came to the Victoria University of Wellington in 2010 through a scholarship from the Ministry of Foreign Affairs, New Zealand to study his Ph.D program. He will serve NCHMF right after he complete his Ph.D. 